\title{
Fluktuationen in Membranen - Schallgeschwindigkeitsmessungen, Kalorimetrie sowie dielektrische und akustische Spektroskopie an wäßrigen Phospholipidsuspensionen
}

\author{
Dissertation \\ zur Erlangung des Doktorgrades \\ der Mathematisch-Naturwissenschaftlichen Fakultäten \\ der Georg-August-Universität zu Göttingen
}

vorgelegt von

Wilfried Schrader

aus Warburg

Göttingen 2001 
D 7

Referent:

Korreferent:

Tag der mündlichen Prüfung:
Prof. Dr. R. Ronneberger

Prof. Dr. W. Schröter

19. April 2001 


\section{Inhaltsverzeichnis}

1 Einleitung $\quad 5$

2 Membranen, Phasenübergänge 9

2.1 Membranen . . . . . . . . . . . . . . . . . . . . . 9

2.2 Phasenübergänge . . . . . . . . . . . . . . . . . . . . 10

2.3 Mischungsverhalten . . . . . . . . . . . . . . . . . . . . 12

2.4 Die Meßsubstanzen und ihre Eigenschaften . . . . . . . . . . . . . . 13

2.5 Präparation . . . . . . . . . . . . . . . . . . . . 18

2.6 Zusammensetzung der verwendeten Suspensionen . . . . . . . . . . 21

3 Schallgeschwindigkeitsmessung/ Kalorimetrie 25

3.1 Kalorimetrie - Meßverfahren . . . . . . . . . . . . . . . . 25

3.2 Kalorimetrie - Ergebnisse . . . . . . . . . . . . . . . 26

3.3 Schallgeschwindigkeitsmessung - Meßverfahren . . . . . . . . . . . 30

3.4 Schallgeschwindigkeitsmessung - Ergebnisse . . . . . . . . . . 33

3.5 Die Theorie von T. Heimburg . . . . . . . . . . . . . . . . . . . 39

3.6 Kompressibilität und Fluktuationen . . . . . . . . . . . . . . . . . . . . . . . 44

3.7 Strukturelles Verhalten . . . . . . . . . . . . . . . . . . . . 47

4 Dielektrische Spektroskopie $\quad \mathbf{4 9}$

4.1 Flüssigkeiten in elektrischen Wechselfeldern . . . . . . . . . . . . . . . 49

4.2 Relaxationsmodelle . . . . . . . . . . . . . . . . . . 51

4.3 Reflexionsfaktormeßplatz . . . . . . . . . . . . . . 53

4.4 Die Spektren . . . . . . . . . . . . . . . . . . . . 57

4.5 Parameter der Anpassung . . . . . . . . . . . . . . . . 65

4.6 Die Theorie von R. Pottel . . . . . . . . . . . . . . . . . 71

4.7 Ergebnisse der Anpassung der Theorie . . . . . . . . . . . . . . 75

5 Grundlagen der Ultraschallspektroskopie 83

5.1 Klassische Dämpfung . . . . . . . . . . . . . . . . . 83

5.2 Zusätzliche Dämpfung . . . . . . . . . . . . . . . . 84

5.3 Kritisches Verhalten . . . . . . . . . . . . . . . . . . 85

5.4 Theorie von Bhattacharjee und Ferrell . . . . . . . . . . . . . 86

5.5 Andere Modelle . . . . . . . . . . . . . . . . . . . . . . 88

6 Meßverfahren der Ultraschallspektroskopie $\quad 91$

6.1 Das Resonatorverfahren . . . . . . . . . . . . . . . . . . 91

6.2 Das Schwingungspulstransmissionsverfahren . . . . . . . . . . . 100

$\begin{array}{lll}7 & \text { Meßergebnisse der Ultraschallspektroskopie } & 109\end{array}$

7.1 Darstellung der Spektren . . . . . . . . . . . . . . . . . . . . . . 109

7.2 Der Fluktuations-Term . . . . . . . . . . . . . . . . . . . 130

7.3 Mögliche molekulare Ursachen der Debye-Terme . . . . . . . . . . . . 145

7.4 Der tieffrequente Debye-Term . . . . . . . . . . . . . . . . . 146

7.5 Der hochfrequente Debye-Term . . . . . . . . . . . . 155 
7.6 Der B-Wert . . . . . . . . . . . . . . . . . . 167

8 Zusammenfassung $\quad 169$

$\begin{array}{ll}\text { Literatur } & 173\end{array}$

$\begin{array}{ll}\text { Danksagung } & 179\end{array}$

$\begin{array}{ll}\text { Lebenslauf } & 181\end{array}$ 


\section{Einleitung}

Es ist seit langem bekannt, daß Pflanzen und Tiere aus einzelnen Zellen bestehen. Selbst in einem einfachen Lichtmikroskop sind diese Zellen als kleine unterteilte Einheiten zu sehen, die durch sogenannte Membranen abgeschlossen werden. Die äußere Zellmembran erfüllt dabei vielfältige Funktionen und Aufgaben. Durch sie wird ein definiertes Volumen geschaffen, in dem sich andere funktionale Einheiten aufhalten und chemische Reaktionen stattfinden können [136]. Membranen existieren nicht nur als äußere begrenzende Hülle der Zellen, sondern bilden auch die begrenzenden Schichten der einzelnen Organellen innerhalb der Zelle wie den Mitochondrien, dem endoplasmatischen Retikolum oder dem Zellkern. Doch die Membran hat neben der passiven Aufgabe der begrenzenden Schicht zwischen Zelle und Außenwelt auch noch aktive Funktionen. So laufen beispielsweise wichtige Energieumwandlungsprozesse wie die Photosynthese und die ATP-Gewinnung an Membranen ab.

Biologische Membranen bestehen aus unterschiedlichen Molekülen. Eine evident wichtige Molekülgruppe sind die Lipide, die ausgeprägte amphiphile Eigenschaften besitzen, da sie aus einem hydrophilen Kopf und einem langgestrecktem, hydrophoben Kohlenwasserstoffteil bestehen. Dabei ordnen sich diese Moleküle in wäßriger Lösung und über ein weites Konzentrationsverhältnis so als Doppelschicht an, daß die äußere Schicht der Membran durch die hydrophilen Kopfgruppen gebildet wird, und der innere Teil der Membran durch die langgestreckten, hydrophoben Kohlenwasserstoffketten ausgefüllt wird. Die wichtigsten Lipide in tierischen Membranen sind Diacylphosphatidylcholin, Diacylphosphatidyläthanolamin und Diacylphosphatidylserin, während in der Pflanzenwelt neben den Phospholipiden vorwiegend Galactolipide vorkommen, bei denen die polaren Gruppen durch Zuckermoleküle (Glucose) gebildet werden [126]. Daneben enthalten biologische Membranen weitere Moleküle wie Proteine mit spezifischen funktionellen Eigenschaften. Proteine können z.B. die Konzentration von $\mathrm{Ca}^{+}$-Ionen in der Zelle definiert steuern. Es existieren ebenfalls sog. periphere Proteine, die nicht die gesamte Membran durchstoßen, sondern ausschließlich entweder auf der Innenseite, oder Außenseite zu finden sind. Sie dienen u.a. der Identifikation der Zelle. Antikörper oder andere Informationen transportierende Moleküle des Organismus sind in der Lage sich an diese Proteine anzulagern. Weiter besitzt die lebende Zelle die Fähigkeit, durch Änderung der Zusammensetzung der Membran eine Zellteilung definiert zu steuern.

Um die physikalischen Eigenschaften dieser Systeme zu studieren, greift man in der Wissenschaft zunächst auf Modellmembranen zurück, die den biologischen Membranen sehr ähnlich sind, jedoch im Gegensatz zu ihnen nicht aus bis zu hundert verschiedenen, sondern nur aus einigen wenigen Lipiden bestehen. Die meisten dieser Doppelschichten zeigen einen thermotropen kristallin $\rightarrow$ flüssig kristallinen Phasenübergang. Die Umwandlung ist durch die thermisch induzierte Bildung von Rotationsisomerien in den Kohlenwasserstoffketten der Lipide verursacht. Die Bildung hoch beweglicher Kinken oberhalb der Phasenumwandlung führt zu einer hohen Fluidität der Membran, jedoch ohne daß die äußere Gestalt der lammellaren Doppelschicht zerstört wird. Dieser Prozeß der Phasenumwandlung wird allgemein als schwacher zweiter Ordnung (weak second Order) bezeichnet und verläuft nicht plötzlich sondern die entsprechenden thermodynamischen Größen zeigen über der Temperatur einen stetigen Verlauf. Dabei treten während der Phasenumwandlung starke Fluktuationen diverser thermodynamischer Parameter auf, was sich u.a. in 
der Bildung von Domänen, bestehend aus Lipiden entweder der kristallinen Phase oder der flüssigen Phase, äußert [68]. Es stellt sich hierbei sofort die Frage der Relevanz dieses Effektes für die Biologie. In biologische Membranen sind, wie oben erwähnt, fast immer funktionale Moleküle, wie z.B. Proteine eingelassen. Je nach Dimension und Größe dieser funktionalen Moleküle fügen sie sich eher in eine Umgebung bestehend aus Lipiden der kristallinen Phase oder der flüssigen Phasen ein. So kann es, bei geeigneten Eigenschaften der Membran, zu Clusterbildung der Proteine kommen, was natürlich Auswirkungen auf die gesamte Funktionalität der Membran hat [54]. In realen biologischen Membranen sind neuerdings die in Modellmembranen vorkommenden Domänen [21] [113] [90] ebenfalls nachgewiesen worden [134] [20], wodurch die Fluktuationen und das Phasenverhalten biologischer Systeme in weitere Aufmerksamkeit rückt.

Im weiteren Umfeld dieser Systeme ergeben sich Fragestellung, wie sich die Zusammensetzung von Membranen, bestehend aus zwei unterschiedlichen Typen von Lipiden, im Einzelnen in deren Phasenverhalten ausdrückt. Mit Hilfe von Schallgeschwindigkeits- und Dichtemessungen [41] [106] [107] ist man in der Lage die Kompressibilität in Abhängigkeit der Temperatur mit hoher Genauigkeit zu bestimmen [97]. Aufgrund zunehmender Fluktuationen des Volumens bei Annäherung an den Phasenübergang ist ein starkes Ansteigen der Kompressibilität zu erwarten. Mit diesem Verfahren sind Aussagen über die Intensität der Fluktuationen möglich, und es ist die Breite und damit die Kooperativität des Phasenübergangs verschiedenartiger Mischungen von Lipiden zu ermitteln. Ergänzend dazu sind kalorimetrische Messungen zu nennen [56] [22], bei denen die Umwandlungswärme des Phasenübergangs direkt durch die temperaturabhängige Wärmekapazität zu messen ist. Es soll versucht werden, die Ergebnisse beider Meßverfahren mittels einer entsprechenden Theorie von T. Heimburg [53] [49] ineinander umzurechnen und so die Ergebnisse beider Verfahren quantitativ miteinander zu verknüpfen.

Bei den bisherigen Betrachtungen steht entweder die gesamte Membran oder der Fragen im Blickpunkt, ob die Kohlenwasserstoffketten die Eigenschaften der Membran beeinflussen. Nachdem es in den 80er Jahren erste Messungen der Umlagerung der dipolaren Kopfgruppen mittels dielektischer Spektroskopie gegeben hat [120] [71] [79] [46] [57], und heute verbesserte und genauere Meßmethoden zur Verfügung stehen, sind Fragestellungen interessant, inwiefern die Fluktuationen und die Bildung von Domänen die Beweglichkeit der Kopfgruppen beeinflußt. Besonders interessant ist die Frage, ob die in der Umwandlung herrschende erhöhte Korrelation der Wechselwirkungen der Lipide untereinander sich auf die Kopfgruppenumlagerung auswirkt. Des weiteren werden Mischungen von Lipiden im Hinblick auf die Richtungskorrelation der Kopfgruppenumlagerung untersucht.

Es deutet sich durch biologische und physikalische Forschungsergebnisse mehr und mehr an, daß in der Biologie fast jeder molekularer Prozeß eine spezifische Funktion besitzt. Deshalb ist es von besonderem Interesse, die molekularen Vorgänge und deren zeitliches Verhalten zu verstehen. Ein möglicher Zugang dazu ist die Ultraschallspektroskopie, mit der sowohl Einzelzeitrelaxationsphänomene als auch die Dynamik der Fluktuationen thermodynamischer Zustände detektierbar sind [51] [135] [109] [108] [78] [50] [100] [40] [110] [67]. Durch die Möglichkeit, sehr breitbandige Spektren $(200 \mathrm{kHz}$ bis $2 \mathrm{GHz}$ ) aufnehmen zu können, ist dies für einen weiten Bereich zeitlichen Verhaltens gegeben. Das Auftreten von Fluktuationen und 
Domänen führt zu einem charakteristischen Anstieg der Ultraschalldämpfung in der Phasenumwandlung. Untersuchungsgegenstand dabei sind Membranen bestehend ausschließlich aus Dimyristoylphosphatidylcholine, Mischungen aus Dimyristoylphosphatidylcholine und Dipalmytoyl-Phosphatidylcholine und Mischungen aus Dimyristoylphosphatidylcholine und Cholesterin.

Ziel dieser Arbeit ist das bessere Verständis von Relaxationsphänomenen, des Phasenverhaltens von Phospholipidmembranen und den dabei auftretenden Fluktuationen der Ordnungsparameter. Es soll die Anwendbarkeit der Theorie von Bhattacharjee und Ferrell für kritisch entmischende Flüssigkeiten auf die Ultraschallabsorption von Lipidsuspensionen untersucht werden. Ebenfalls soll das zeitliche Verhalten von in diesem Zusammenhang wichtigen molekularen Prozessen wie Kinkenbildung der Alkylketten untersucht werden. Weiter soll die Anwendbarkeit der Theorie von T. Heimburg, die einen Zusammenhang zwischen Wärmekapazität und Kompressibilität der Lipidsysteme herstellt, auf diverse Lipidmischungen im fluktuativen Zustand untersucht werden. Schließlich soll ein sicherer Nachweis der Umorientierung der Kopfgruppen der Lipidmoleküle und ein Studium der gesamten Relaxationsdynamik der Kopfgruppenumorientierung in Lipidmembranen unterschiedlicher Zusammensetzung getätigt werden. 


\section{Membranen, Phasenübergänge}

\subsection{Membranen}

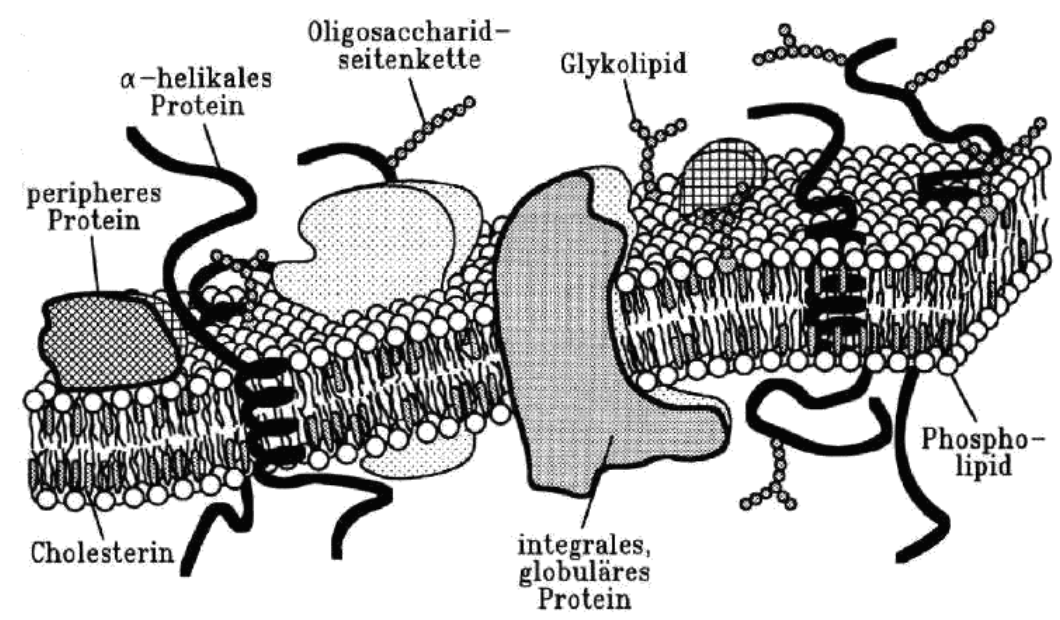

Abb. 2.1: Modell einer biologischen Membran; sie enthält neben Lipiden diverse funktionale Moleküle

Membranen bestehen zum großen Teil aus den sogenannten Lipiden. Biologische Membranen, wie sie auch im menschlichen Körper vorkommen, bestehen aus bis zu einhundert verschiedenen Lipidtypen. Die in dieser Arbeit betrachteten Lipide sind zwitterionische Moleküle, die einen langkettigen, aus Kohlenstoffketten bestehenden hydrophoben Teil und einen polaren Kopf besitzen. Der Kopf fügt sich wegen seiner elektrischen Ladungen sehr gut in die von dipolaren Wassermolekülen Umgebung ein. Dagegen ist der hydrophobe Teil bestrebt, möglichst wenig dem Wasser zugewandt zu sein. Aufgrund dieser Eigenschaften ist es energetisch günstiger für die Lipidmoleküle - entropisch für das Wasser - , wenn sie sich in einer Lipiddoppelschicht anordnen, deren Äußeres mittels der polaren Köpfe dem Wasser zugewandt ist und deren Inneres durch die hydrophoben Kohlenwasserstoffketten gebildet wird (siehe Abb. 2.1) [127]. Befinden sie sich in wäßriger Lösung, nehmen die Lipide spontan eine solche Konfiguration ein. Es handelt sich somit um eine gewisse Selbstorganisation der Moleküle [44].

In der Biophysik betrachtet man nun zunächst keine solch komplexen Membranen, wie sie in der Natur vorkommen, sondern beschränkt sich auf Modellmembranen, deren Zusammensetzung genau bekannt ist. Die am einfachsten denkbare Konstellation ist eine Membran bestehend aus nur einem Lipidtyp. Die Herstellung einer solchen künstlichen Membran ist durch die Selbstorganisation der Lipide zu Membranen in wäßriger Lösung vergleichsweise einfach.

Ordnen sich also Lipide zu einer Membran zusammen so bilden sie einen sogenannten Flüssigkristall. Ähnlich wie andere Stoffe, besitzt auch Lipid eine Schmelztemperatur. Oberhalb einer spezifischen Temperatur werden die langkettigen Kohlenstoffketten zu rasch fluktuierenden Rotationsisomerien und Kinkenbildung angeregt. In einer Membran spielen zwischenmolekulare Wechselwirkungen und die Kooperativität der Fluktuationen einzelner Moleküle eine große Rolle und bestimmt das Phasenverhalten der gesamten Membran. Einige in dieser Arbeit betrachteten Lipidmembranen 


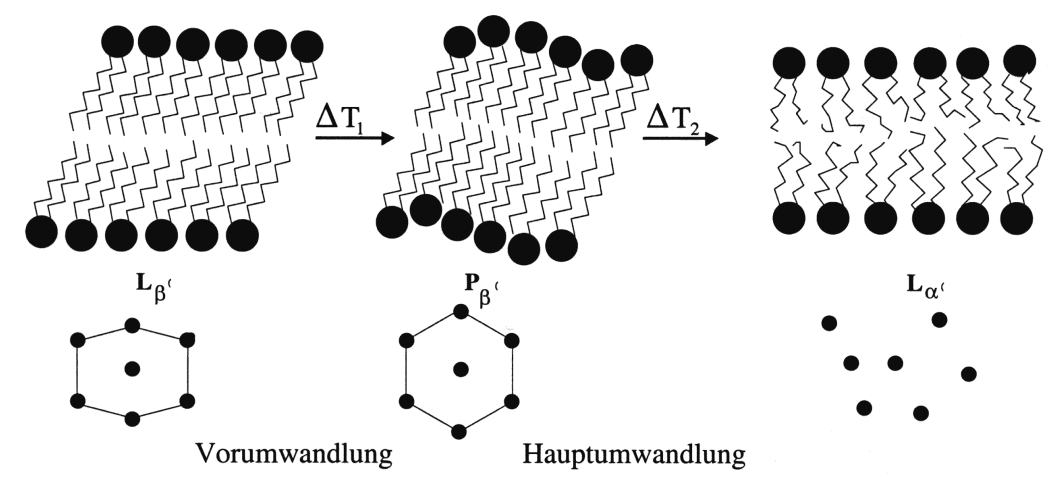

Abb. 2.2: Schematische Darstellung von Vor- und Hauptumwandlung in einer Lipiddoppelschicht. Die Umwandlungen zeichnen sich durch eine Umlagerung der Kopfgruppen, eine Konformationsänderung und eine Änderung der Nahordnung in der Membranebene aus.

(z.B. DMPC) besitzen eine charakteristische Vorumwandlungstemperatur und eine Hauptumwandlungstemperatur, die um ca. $10{ }^{\circ} \mathrm{C}$ höher liegt. Unterhalb der niedrigeren Umwandlungstemperatur liegt die Membranen in der sogenannten "flüssig - kristallinen" $\mathrm{L}_{\beta^{\prime}}$-Phase vor. Sie zeichnet sich durch ein hohes Maß an Ordnung aus. Die Moleküle sind dicht gepackt und weisen in lateraler Richtung eine hexagonale Struktur auf. Die langkettigen Kohlenwasserstoffketten sind leicht gegen die Membrannormale geneigt. In der Vorumwandlung geht der Zustand der Membran in einen weiteren "flüssig - kristallinen" Zustand über, die $\mathrm{P}_{\beta^{\prime}}$-Phase. Sie unterscheidet sich von der vorherigen nur durch eine zusätzliche wellenartige, die Membran durchziehende Struktur. Die hexagonale Struktur wird dabei ein wenig verzerrt. Bei einer weiteren Temperaturerhöhung wird die Hauptumwandlung erreicht, bei der in den Ketten schnelle ungeordnete Drehungen der $\mathrm{CH}_{2}$-Segmente um die C-C-Bindungen angeregt werden. Dieser "flüssige" Zustand der Membran wird $\mathrm{L}_{\alpha^{\prime}}$-Phase genannt. Diese verschiedenen Phasenzustände sind schematisch in Abb. 2.2 dargestellt.

\subsection{Phasenübergänge}

Im allgemeinen wird einen Übergang zweiter Ordnung für demn oben beschriebenen Phasenübergang einer Lipidmembran angenommen. Diese Annahme wird stark durch kalorimetrische Messungen und Monte-Carlo-Simulationen, die die kalorimetrischen Messungen sehr gut wiedergeben können, unterstützt [54] [13] [68] [56].

Die zentrale Größe zur theoretischen Beschreibung von Phasenübergängen ist der Ordnungsparameter $\phi$. Er ist für jeden betrachteten Phasenübergang entsprechend geeignet zu wählen. Phasenübergänge können nun anhand von $\phi$ in zwei Klassen eingeteilt werden:

1. Bei einem Phasenübergang erster Ordnung ändert sich der Ordnungsparameter $\phi$ am Phasenübergang unstetig.

2. Phasenübergänge, bei denen sich der Ordnungsparameter am Phasenübergang stetig ändert, aber deren Ableitung unstetig ist, werden Phasenübergänge zweiter Ordnung genannt. 
Der Prozeß des Phasenübergangs zweiter Ordnung besitzt charakteristische Eigenschaften, die hier kurz erwähnt werden sollen [115]:

- Es wird für den Übergang von der einen zur anderen Phase keine Umwandlungswärme benötigt.

- Es tritt keine Koexistenz beider Phasen während des Phasenüberganges auf.

- Die Entropie S(T,p) und das Volumen V(T,p) sind am Umwandlungspunkt stetig.

- Die isobare Wärmekapazität $C_{p}$, die isotherme Kompressibilität $\kappa_{T}$ und die isobare Kompressibilität $\kappa_{P}$ zeigen am Phasenübergang ein unstetiges Verhalten.

Der Phasenübergang zweiter Ordnung zeichnet sich zudem durch Fluktuationen während des Phasenüberganges aus. Dies führt zu einem gänzlich anderen Verhalten, als es bei den Übergängen erster Ordnung der Fall ist, und wird als kritisches Verhalten bezeichnet. Dieses Verhalten wird mittels der phänomenologischen Ginzburg-Landau Theorie beschrieben, wonach die freie Energie F des Systems in der Nähe des Phasenübergangs, wegen des kontinuierlichen Verschwindens des Ordnungsparameters $\phi$, um $\phi=0$ entwickelt werden kann:

$$
F=F_{0}+\int d^{n} \vec{r}\left(a_{2} \phi^{2}+a_{4} \phi^{4}+g(\nabla \phi)^{2}\right) \quad .
$$

Dabei ist $F_{0}$ die freie Energie für $\phi=0$ und $a_{2}, a_{4}, g$ sind Entwicklungskoeffizienten. Das alleinige Auftreten von geraden Potenzen von $\phi$ ist eine Folge der Symmetrie des Systems. Im Falle der Hauptumwandlung einer Lipidmembran sind z.B. $\phi=$ $\rho_{\text {gel }}-\rho_{\text {fluid }}$ und $\phi=\rho_{\text {fluid }}-\rho_{\text {gel }}$ als äquivalente Definitionen anzusehen ( $\rho$ ist die Dichte des Systems).

Üblicherweise betrachtet man den Phasenübergang in Abhängigkeit von der Temperatur. Es gilt

$$
a_{2} \sim \frac{T-T_{c}}{T_{c}}
$$

mit der kritischen Temperatur $T_{c}$.

Es lassen sich einem System, welches einem Phasenübergang zweiter Ordnung unterliegt, folgende wichtige Eigenschaften zuschreiben:

- In einem engen Bereich um die Umwandlungstemperatur $T_{c}$ herum divergiert die Korrelationslänge $\xi$. Sie ist dort wesentlich größer als die sonst üblichen effektiven Reichweiten, die in der Regel einige Atomabstände betragen. Mangels einer ausgezeichneten Längenskala wird das gesamte System skaleninvariant.

- Alle thermodynamischen Größen "gehorchen" in der Nähe des kritischen Punktes Potenzgesetzen, die die Form

$$
f \sim\left|\frac{T-T_{c}}{T_{c}}\right|^{\gamma}
$$


haben. Die Größe $\gamma$ wird als kritischer Exponent bezeichnet. Dieses Verhalten ist eine Folge der Skaleninvarianz des Systems.

- Die thermodynamischen Antwortfunktionen isobare Wärmekapazität $C_{p}$, isotherme Kompressibilität $\kappa_{T}$ und isobare Kompressibilität $\kappa_{T}$ divergieren am kritischen Punkt in einem Potenzgesetz. Das Verhalten von $C_{p}$ und $\kappa_{T}$ steht über das Fluktuations-Dissipations-Theorem mit den Fluktuationen des Systems in Verbindung:

$$
\begin{aligned}
C_{p} & =\left(\frac{d \bar{H}}{d T}\right)_{p}=\frac{\bar{H}^{2}-\bar{H}^{2}}{R T^{2}}, \\
\kappa_{T} & =-\frac{1}{\bar{V}}\left(\frac{d \bar{V}}{d p}\right)_{T}=\frac{\overline{V^{2}}-\bar{V}^{2}}{\bar{V} \cdot R T} .
\end{aligned}
$$

Die Wärmekapazität $C_{p}$ und $\kappa_{T}$ sind somit um so größer, je größer auch die Fluktuationen in der Enthalpie $H$ bzw. des Volumens $V$ sind.

- Am kritischen Punkt wird die gesamte Dynamik des System sehr langsam. Dieses Phänomen wird als critical slowing down bezeichnet. Die dynamische Erweiterung der Landau-Theorie beschreibt dieses Verhalten [61]. Unter Vernachlässigung der nichtlinearen Anteile gilt danach:

$$
\partial_{t} \phi(x, t)=\Gamma \nabla^{2} \frac{\delta F}{\delta \phi} \approx 2 \Gamma a_{2} \nabla^{2} \phi(x, t)
$$

Die Lösung dieser Differentialgleichung im Fourierraum ist

$$
\phi(x, t) \sim e^{-\frac{t}{\tau}} \quad \text { mit } \quad \tau=\frac{1}{2 \Gamma a_{2} k^{2}}
$$

Dabei ist $k$ die Wellenzahl und $\Gamma$ der kinetische Koeffizient. Die Gleichungen (2.6) und (2.7) zeigen, daß die charakteristische Zeitskala $\tau$ im Umwandlungspunkt $T \rightarrow T_{c}$ (d.h. $a_{2} \rightarrow 0$ ) divergiert. Somit wird die Dynamik des System sehr langsam.

\subsection{Mischungsverhalten}

Die Lipidmembranen werden durch einen Extrusionsvorgang in eine Kugelform, Vesikel genannt, mit definiertem Durchmesser gebracht (siehe auch Kap. 2.5). In diesen Vesikeln ist eine gewisse Menge Wasser gespeichert. Die Suspensionen stellen somit ein ternäres System, bestehend aus Wasser im Vesikelkern, Doppelschicht und suspendierendem Wasser dar. Wegen der auftretenden Dichtefluktuationen in der Membran kommt es während der Hauptumwandlung zu einer Veränderung der Dimensionen der Membran [26]. Es ist jedoch beobachtet worden, daß hauptsächlich Beiträge von Isomerisierungsprozessen der Alkylketten und Wechselwirkungen zwischen diesen Ketten zur Umwandlungsenthalpie beitragen [16], während alle anderen Effekte wie Kopfgruppenumlagerung und Hydratation vernachlässigbar sind. Zudem 
wird wegen neusten Drucksprungexperimenten vermutet, daß die Relaxationszeiten, die den Wärmefluß des suspendierenden Wassers in die Membran beschreiben, im Bereich von einigen Sekunden liegt [67], was deutlich höher als die in dieser Arbeit betrachteten Relaxationszeiten liegt. Aus diesem Grund kann das Membran-Wasser System in guter Näherung als ein pseudobinäres System betrachtet werden [41] und ist mittels der Thermodynamik für das Phasenverhalten echter binärer Mischungen beschreibbar. Weiter können molekulare Vorgänge innerhalb der Membran losgelöst vom dem umgebenden Wasser betrachtet werden.

\subsection{Die Meßsubstanzen und ihre Eigenschaften}

\subsubsection{DMPC}
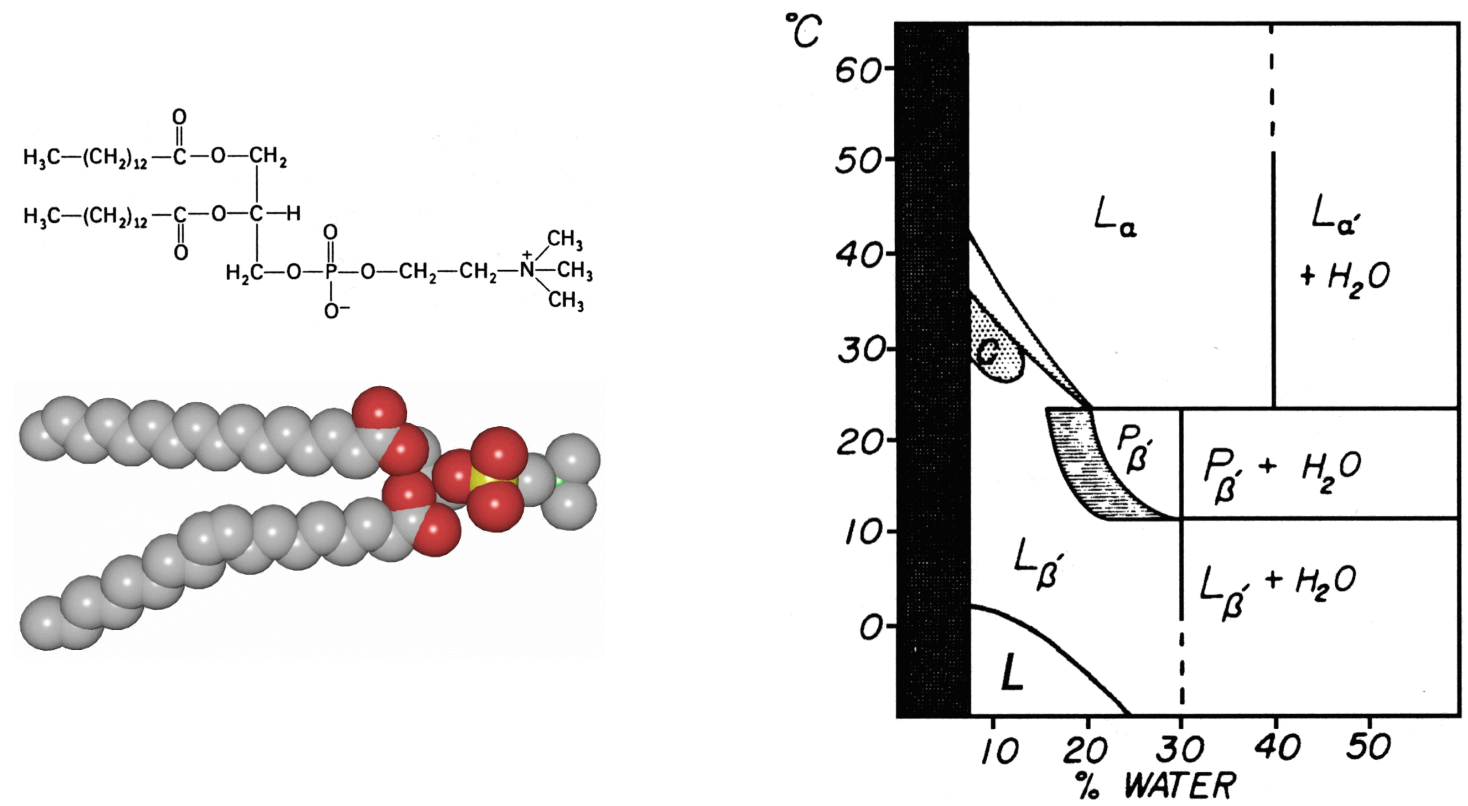

Abb. 2.3: Struktur von DMPC (links), Phasendiagramm DMPC/Wasser (rechts) nach [69]

1,2-Dimyristoyl-L-3-phosphatidylcholin (DMPC) mit der Summenformel $\mathrm{C}_{36} \mathrm{H}_{72} \mathrm{NO}_{8} \mathrm{P}$ (siehe auch Abb. 2.3) und der Molmasse von 677,9 $\frac{\mathrm{g}}{\mathrm{mol}}$ ist ein typisches Phospholipid. Solch ein Phospholipid besteht aus einem Glycerinteil, von dessen Hydroxylgruppen zwei mit Fettsäureketten und die dritte, hier die sn-3 Gruppe, mit einem Phosphatrest mit angeschlossener Alkoholgruppe verestert sind. Der wasserliebende Kopf des Moleküls besitzt ein elektrisches Dipolmoment, wobei der Cholinteil positiv geladen und der Phosphatteil negativ geladen ist. Die langkettigen Fettsäuren bestehen bei aus zahlreichen $\mathrm{CH}_{2}$ Gruppen, die bei DMPC aus 14 Kohlenstoffatomen bestehen. Aufgrund des amphiphilen Charakters dieses Phospholipides zeigt dieses Molekül in wäßriger Lösung ein sehr komplexes Phasenverhalten.

In Abb. 2.3 ist ein solches Phasendiagramm von DMPC/Wasser dargestellt. Je nach Wassergehalt und Temperatur bildet das System verschiedene Phasen aus. Z.B. existiert die kubisch-mizellare, die hexagonale und die lamellare Phase, von denen ich 
jedoch nur auf die lamellare Phase eingehen möchte, da alle anderen möglichen Phasen für diese Arbeit nicht weiter relevant sind. Ab einem bestimmten Wassergehalt der Mischung liegen die Lipide nur noch in der sogenannten lammellaren Phase vor und bilden die oben schon beschriebenen Membranen. Auch in der lammellaren Phase sind wie zuvor erwähnt drei verschiedene Phasen möglich. Unterhalb der sogenannten Vorumwandlung liegen die Lipide in der $\mathrm{L}_{\beta}$-Phase, auch gelförmigen Phase vor. Über dieser Temperatur befinden sich die Moleküle in einem Zwischenzustand, der als "ripple-phase" oder $\mathrm{P}_{\beta}$-Phase bezeichnet wird. Über der eigentlichen Umwandlungstemperatur wird die Phase als fluide Phase oder $\mathrm{L}_{\alpha}$-Phase bezeichnet. Die Umwandlungstemperatur von DMPC/Wasser liegt bei ca. $24,3{ }^{\circ} \mathrm{C}$.

\subsubsection{DMPC/DPPC Mischungen}
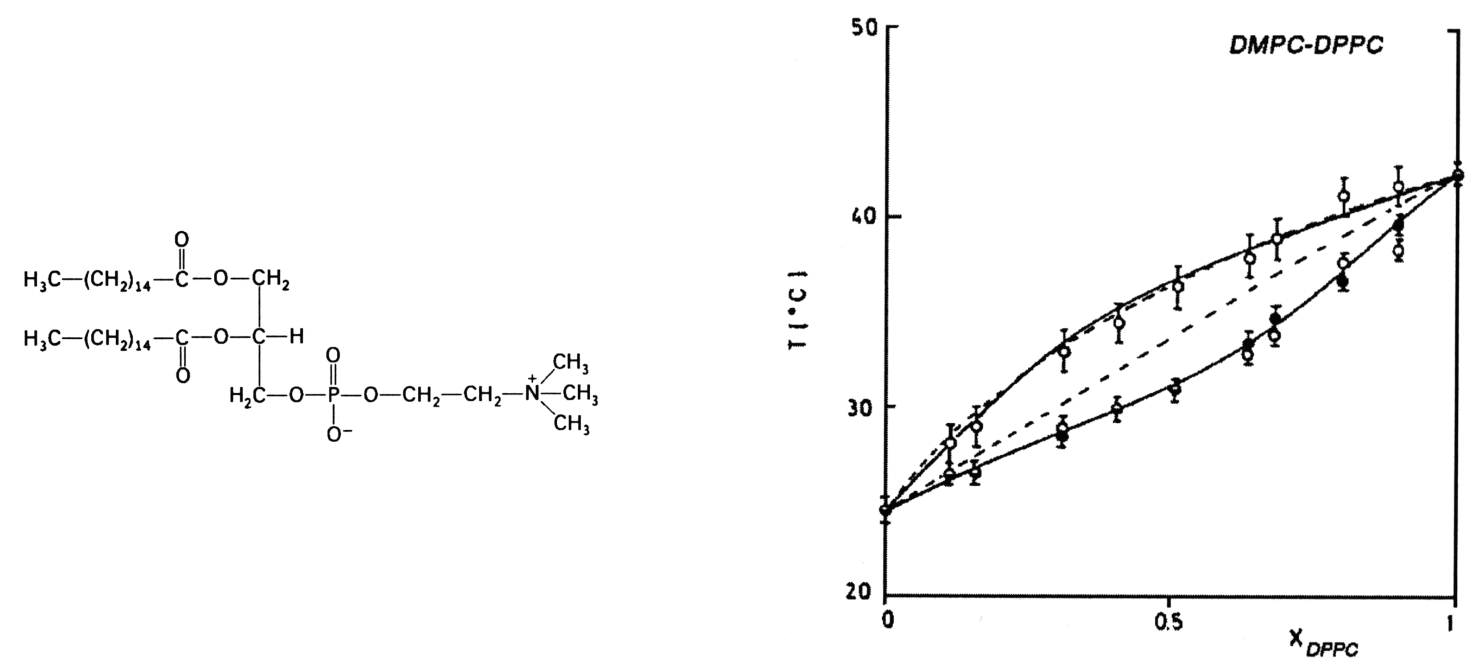

Abb. 2.4: Struktur von DPPC (links), Phasendiagramm DMPC/DPPC in Wasser (rechts) nach [26] $\left(x_{D P P C}\right.$ Molenbruch DPPC), ०: Anisotropie der DPH-Fluorenszenz, •: Lichtstreuung

1,2-Dipalmitoyl-L-3-phosphatidylcholin (DPPC) mit der Summenformel $\mathrm{C}_{40} \mathrm{H}_{80} \mathrm{NO}_{8} \mathrm{P}$ und der Molmasse $734 \frac{\mathrm{g}}{\mathrm{mol}}$ besitzt einen ganz ähnlichen Aufbau, wie das oben beschriebene DMPC. DPPC unterscheidet sich jedoch in der Länge der Fettsäurereste von DMPC. Während DMPC eine Kohlenstoffkettenlänge von 14 aufweist, sind es bei DPPC 16. Dieses führt zu einem vergleichbaren Phasenverhalten der DPPC/Wasser Mischungen zu dem DMPC/Wasser Mischungen, jedoch sind die Umwandlungstemperaturen zu höheren Temperaturen verschoben. DPPC hat, verursacht durch die längeren Kohlenstoffketten, eine Hauptumwandlung bei ca. $42{ }^{\circ} \mathrm{C}$. Aufgrund des gleichen Aufbaus und der chemischen Ähnlichkeit von DMPC und DPPC ist zu erwarten, daß sich Mischungen dieser beiden Lipide gut bilden lassen. Das in Abb. 2.4 dargestellte Phasendiagramm von binären DMPC/DPPC Mischungen läßt auf das Vorliegen einer homogen gemischten Membran schließen. Es bilden sich weder unterhalb noch oberhalb der jeweiligen Umwandlungstemperatur nur aus einer Lipidsorte bestehende Domänen [133] [42]. 

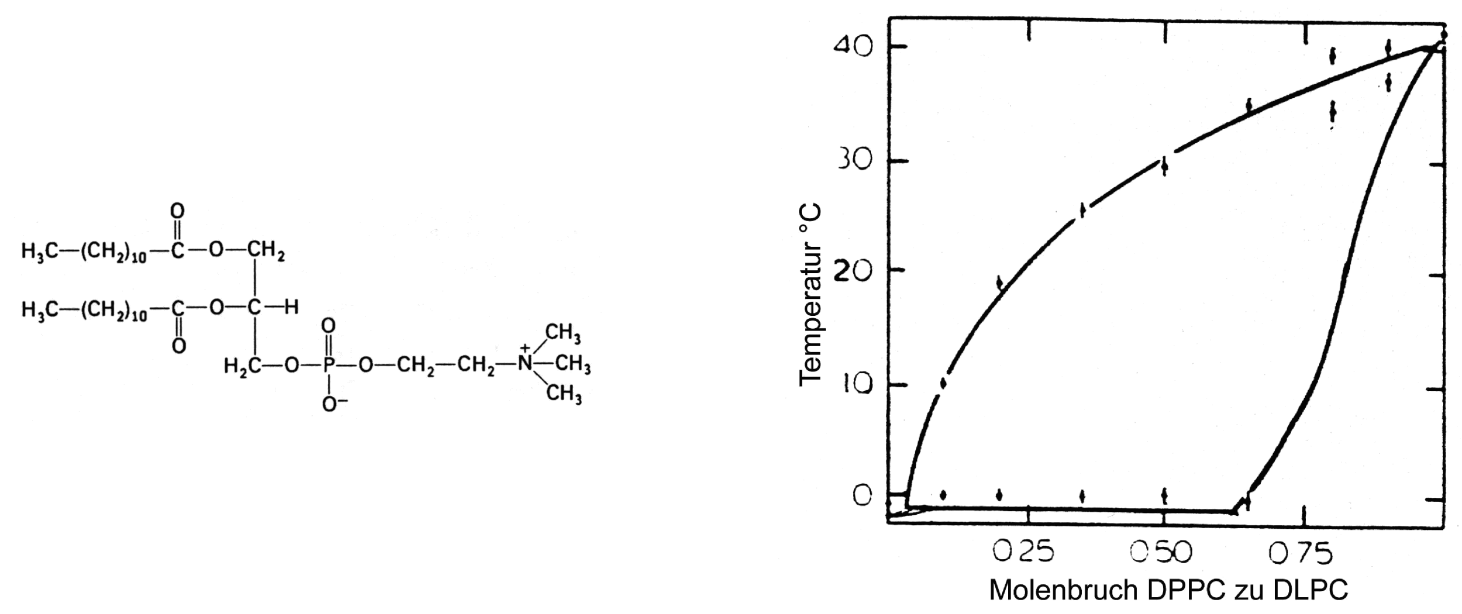

Abb. 2.5: Struktur von DLPC (links), Phasendiagramm DLPC/DPPC in Wasser (rechts) nach [151]

\subsubsection{DLPC/DPPC Mischungen}

1,2-Dilauroyl-L-3-phosphatidylcholin (DLPC) mit der Summenformel $\mathrm{C}_{32} \mathrm{H}_{64} \mathrm{NO}_{8} \mathrm{P}$ und der Molmasse $621,8 \frac{g}{m o l}$ hat erneut einen ähnlichen Aufbau, wie das schon beschriebene DMPC und DPPC. Es besitzt allerdings "nur" Kohlenstoffkettenlänge von 12 und ist damit das Lipid mit den kürzesten Kohlenwasserstoffketten von den hier behandelten. Entsprechend niedrig liegt die Hauptumwandlungstemperatur bei etwa $0{ }^{\circ} \mathrm{C}$. Aufgrund des relativ großen Längenunterschiedes zu DPPC ist es fragwürdig, ob sich beide Lipide ohne weiteres als nahezu ideale Mischung in einer Membran integrieren lassen. Sollte dies nicht der Fall sein, so ergeben sich Fragestellung, inwiefern sich die Entmischung auf das Schmelzverhalten der Ketten und das Phasenverhalten der Membran überträgt. Dies deutet sich schon in dem in Abb. 2.5 dargestelltem Phasendiagramm an, welches einen deutlich breiteren Koexistenzbereich aufweist als bei den DMPC/DPPC Mischungen in Abb. 2.4.

\subsubsection{DMPC/DMG Mischungen}

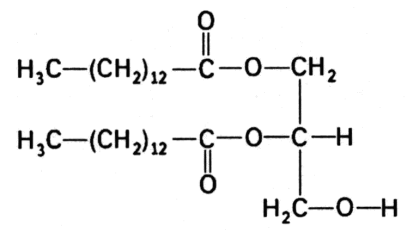
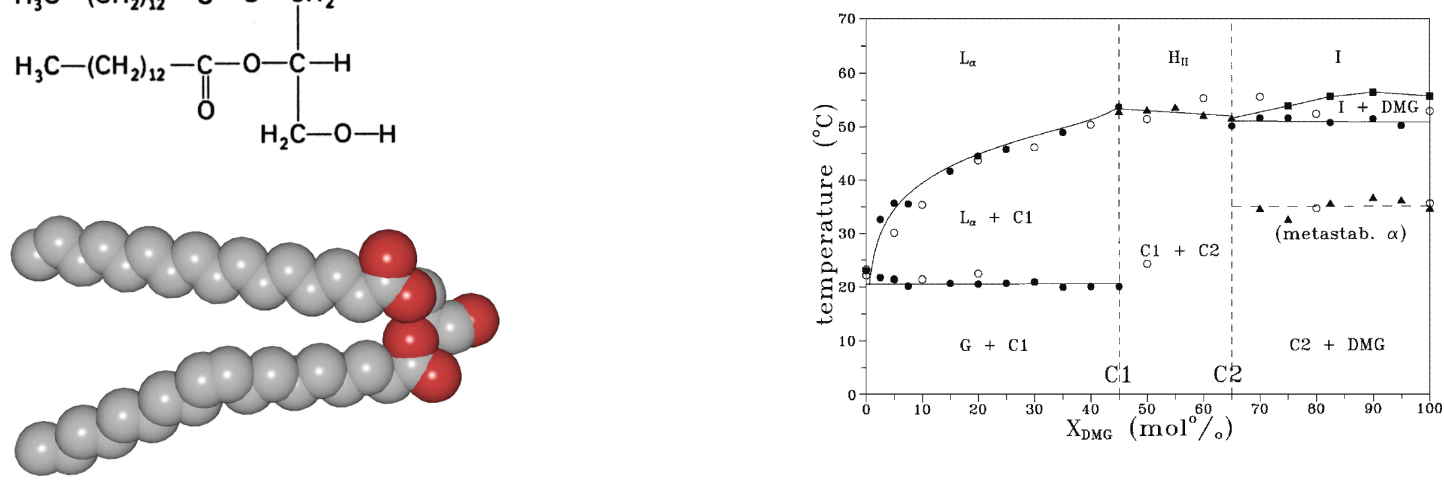

Abb. 2.6: Struktur von DMG (links), Phasendiagramm DMPC/DMG in Wasser (rechts) nach [56] 
Diacylglyceride wie 1,2-Dimyristoyl-rac-Glycerol (DMG), mit der Summenformel $\mathrm{C}_{31} \mathrm{H}_{60} \mathrm{O}_{5}$ und der Molmasse 512,8 $\frac{\mathrm{g}}{\mathrm{mol}}$, werden in der Biologie mit Hilfe des Enzyms Phospholipae C aus Phosphatidylcholin (DMPC) hergestellt [130]. Diacylglyceride aktivieren als sekundärer Botenstoff das Protein Kinase $\mathrm{C}$, welches eine wichtige Rolle bei der Kontrolle der Zellteilung spielt [114]. Diese Aktivierung hängt stark davon ab, wie die Diacylglyceride in die Membran integriert sind. DMG besteht wie DMPC aus zwei Fettsäureresten, welche bei dem hier verwendeten DMG eine Länge von 14 Kohlenstoffatomen aufweist. Beide Kohlenstoffketten sind über ein Glyceringerüst miteinander verbunden und der Glycerinteil ist chemisch durch eine $\mathrm{OH}-$ Gruppe abgeschlossen, welche weit weniger polare Eigenschaften besitzt als die Kopfgruppe bei DMPC. Somit besitzt DMG im Gegensatz zu DMPC und DPPC keinen polaren Kopf sondern nur einen sehr ausgeprägten hydrophoben Teil. Daraus läßt sich direkt die schwere Löslichkeit von DMG allein in Wasser folgern. Jedoch ist relativ leicht möglich, DMG in eine Membran aus DMPC einzubetten. Das Fehlen der Kopfgruppe bei DMG verursacht eine erheblich höhere Kooperativität innerhalb der Membran, als bei den Lipiden mit Kopfgruppe, was sich in einer verhältnismäßig hohen Umwandlungstemperatur niederschlägt. Betrachtet man Mischungen von DMPC und DMG, so ergibt sich durch diese verschiedenartigen Eigenschaften ein recht ungewöhnliches Phasendiagamm. Es sei noch erwähnt, daß bei DMG Anteilen über $45 \%$ der Membran die Lipide nicht mehr in dem gewohnten lateralen Membranaggregat vorliegen ( $L_{\alpha}$-Phase) sondern in der $H_{\alpha}$-Phase, auch hexagonale Phase genannt (siehe Phasendiagramm in Abb. 2.6). Dabei liegen die Lipidmoleküle in langen, zylinderförmigen Röhren vor, die sich ihrerseits hexagonal anordnen. Die Kohlenwasserstoffketten sind dabei in das Innere der Röhren gerichtet.

\subsubsection{DMPC/Cholesterin Mischungen}
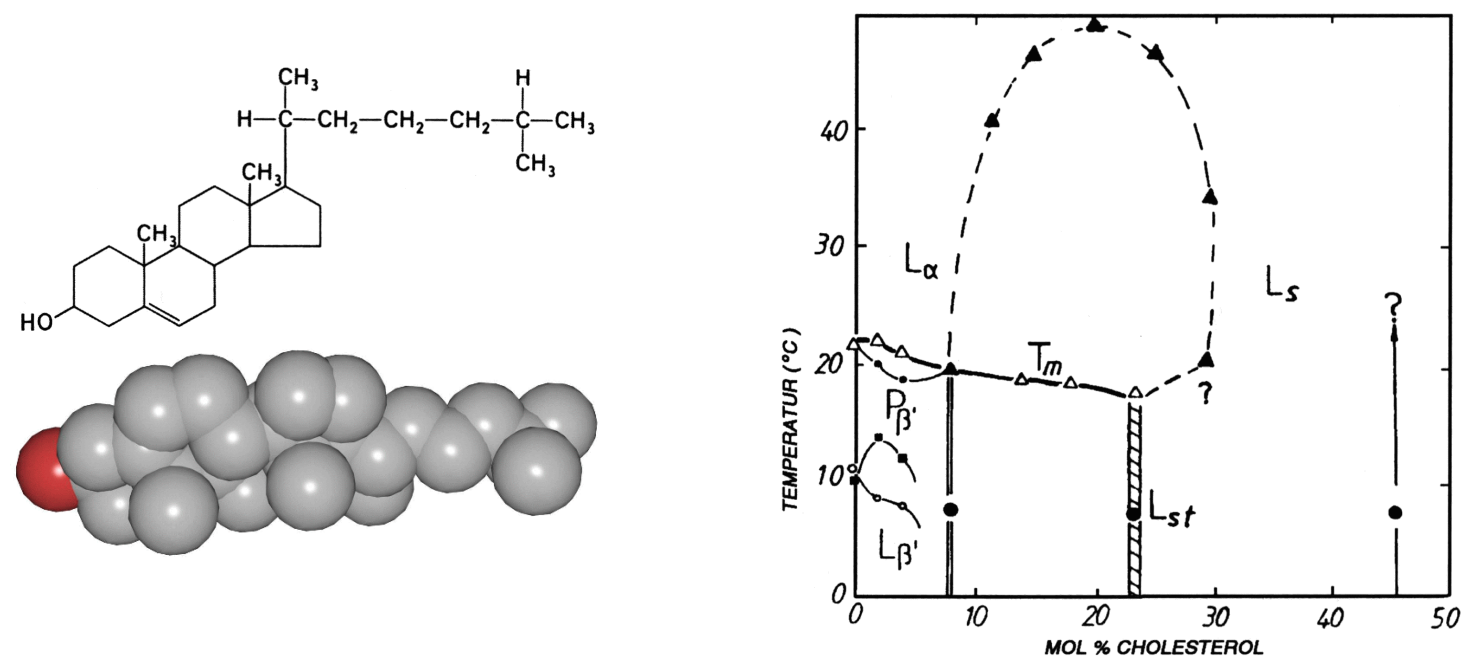

Abb. 2.7: Struktur von Cholesterin (links), Phasendiagramm DMPC/Cholesterin in Wasser (rechts) nach [128]

Eine Lipidmembran aus DMPC verhält sich bei Zugabe von Cholesterin grundsätzlich anders, als bei Zugabe einer Phosphatidylcholin-Komponente, die sich von DMPC 
nur in der Länge der Alkylketten unterscheidet wie DPPC und DLPC. Cholesterin $\mathrm{C}_{27} \mathrm{H}_{46} \mathrm{O}$ mit der Molmasse 386,67 $\frac{\mathrm{g}}{\mathrm{mol}}$ ist ein wichtiger Bestandteil von Membranen des menschlichen Körpers. Es wird eine Wasserstoffbrückenbindung zwischen der OH-Gruppe des Cholesterins und einem Sauerstoffatom der Glycerolgruppe von DMPC angenommen. Dadurch werden die Alkylketten in ihrer Beweglichkeit durch Wechselwirkung mit dem hydrophoben Abschnitt des Cholesterinmoleküls stabilisiert. Cholesterin hat somit einen erheblichen Einfluß auf die Konformation der Moleküle in der Membran und damit auf die Vor- und Hauptumwandlung.

Das Phasendiagramm nach [128] von DMPC/Cholesterin ist in Abb. 2.7 dargestellt. In Abhängigkeit des Zusatzes an Cholesterin stellt es folgendes Verhalten der Membran dar:

- 5 mol \% Cholesterin:

Die Vorumwandlung verschwindet und gleichzeitig wird das Neigen der Alkylketten in der Gel-Phase unterdrückt [30] [101] [102].

- 7,5 mol \% Cholesterin ("eutektischer Punkt"): Es liegt keine Koexistenz von fluider und gelförmiger Phase während der Hauptumwandlung mehr vor. Zusätzlich zur Gel-Phase $L_{\beta^{\prime}}$ und zur fluiden Phase $L_{\alpha}$ tritt eine cholesterinreiche Phase $L_{s}$ auf. Die $L_{s}$-Phase wird als "flüssigkristalline" Phase bezeichnet, da sie sich durch eine besonders hohe Ordnung der Alkylkettenkonformation, verbunden mit einer hohen lateralen Beweglichkeit der Moleküle in der Membran, auszeichnet.

- 7,5 - 22 mol \% Cholesterin: Die Phasen $L_{\beta^{\prime}}, L_{s}$ bzw. $L_{\alpha}$ und $L_{s}$ koexistieren während der Hauptumwandlung nebeneinander.

- 22 - 23 mol \% Cholesterin (zweiter "eutektischer Punkt"): Es existiert nur noch eine einzige Phase $L_{s t}$. Die Hauptumwandlung ist nun fast vollständig unterdrückt.

- 23 - 46 mol \% Cholesterin: Es wird vermutet, daß die Phasen $L_{s}$ und $L_{s t}$ koexistieren, wobei der Cholesteringehalt innerhalb der $L_{s t}$-Phase konstant bleibt.

- > 46 mol \% Cholesterin: Die Membran ist an Cholesterin gesättigt [63]. Eine weitere Erhöhung des Cholesteringehaltes führt zu einer Ausbildung von Domänen reines Cholesterins in der Membran.

Von Halstenberg et al. [49] wurde gezeigt, daß schon eine geringe Zugabe von Cholesterin eine Verbreiterung der Haupt- und Vorumwandlung zur Folge hat. Mit Hilfe der Monte-Carlo-Simulation kann ein vermehrtes Auftreten von Domänen in der Membran gezeigt werden, welche von Cholesterin stabilisiert werden [66] [111] [112] [86]. Cholesterin lagert sich verstärkt an den Domänengrenzen an [43]. Somit wird die Kooperativität vermindert und dies führt zu einer Verbreiterung der Hauptumwandlung [128] [49]. Man kann mittels der Messung der Schallgeschwindigkeit bzw. der Kompressibilität zeigen, daß sich die Kompressibilitäten der gelförmigen und der fluiden Phase mit steigendem Cholesteringehalt annähern. Das kritische Verhalten der Wärmekapazität und der Kompressibilität während der Hauptumwandlung verschwindet zunehmend. 
Einige Proteine lagern sich bevorzugt innerhalb einer Domäne bestimmten Zustandes an. Somit hat die Stabilisierung der Domänen durch die Zugabe von Cholesterin direkten Einfluß auf die Funktionalität der Membran [49].

\subsection{Präparation}

Ziel der aufwendigen Präparation ist es, eine Suspension einschaliger, unilamellarer Vesikel, bestehend aus den beschriebenen Modellmembranen definierter Zusammensetzung, mit möglichst einheitlichem Durchmesser zu erhalten, um Ergebnisse verschiedener Messungen miteinander vergleichbar zu machen. Wichtig dabei ist die Unilamellarität, die eine immer gleichen Umgebung für jede Doppelschichtmembran sicherstellt. Messungen der Wärmekapazität und der Schallgeschwindigkeit von einer Lösung mehrschaliger Vesikel zeigen, daß dieser ineinander befindlichen Membranen nicht getrennt voneinander betrachtet werden können. Eine stark erhöhte Kooperativität ist zu beobachten [53], verusacht durch die Multilammelarität der Vesikel und die entsprechend höheren Radien.

Die Präparation der verwendeten Lösungen gestaltet sich aufwendig und erfordert mehrere Schritte, um das gewünschte Ergebnis - eine Suspension mit Vesikeln einheitlichen Durchmessers von etwa 100nm — zu erhalten. Die Schritte lauten im einzelnen:

1. Als erstes wird das trockene Pulver auf einer Präzisionswaage abgewogen (etwa $50 \mathrm{mg}$ ). Die Waage besitzt eine Genauigkeit von $0,1 \mathrm{mg}$.

2. Um beide Lipidsorten bei mehrkomponentigen Membranen gut zu durchmischen, wird das Pulver in einem organischem Lösungsmittel (Chloroform) aufgelöst. Der Hersteller (Merck) gibt dessen Reinheitsgrad mit 99,0 \% an.

3. Die Chloroformlösung wird in einer Vakuumkammer getrocknet. Es bildet sich ein dünner Film trockenen Lipides an der Gefäßwand.

4. Die gewünschte Menge Wasser wird dem jetzt wieder trockenen Gemisch beigefügt. Die dafür benutzte Waage besitzt eine Genauigkeit von $1 \mathrm{mg}$. Die Phospholipidmoleküle lösen sich im Wasser und bilden mehr oder weniger große Vesikel bzw. ausgedehnte lamellare Strukturen.

5. Die Lösung wird abwechselnd schnell eingefroren und aufgetaut. Die Bildung von Eiskristallen bricht viele Phospholipiddoppelschichten erneut auf und veranlaßt diese, beim Auftauen neue Vesikel zu bilden.

6. Um nun einschalige Vesikel mit einem definierten Durchmesser von $100 \mathrm{~nm} \mathrm{zu}$ erhalten, wird die Flüssigkeit extrudiert. Dabei wird die Lösung unter einem Druck von ca. $4 \frac{\mathrm{kp}}{\mathrm{cm}^{2}}$ und über der Hauptumwandlungstemperatur der jeweiligen Lipidmischung durch einen Membranfilter mit einer Porengröße von 100 nm Durchmesser gepreßt. Dieses sollte ca. 15 bis 20 mal geschehen, um eine möglichst enge Größenverteilung der Vesikel zu erhalten.

Die verwendeten Filter werden von der Firma Poretics Corporation, 111 A Lindbergh Ave., Livermore, CA 94550 bezogen und sind in Tabelle 2.1 zusammengefaßt. 


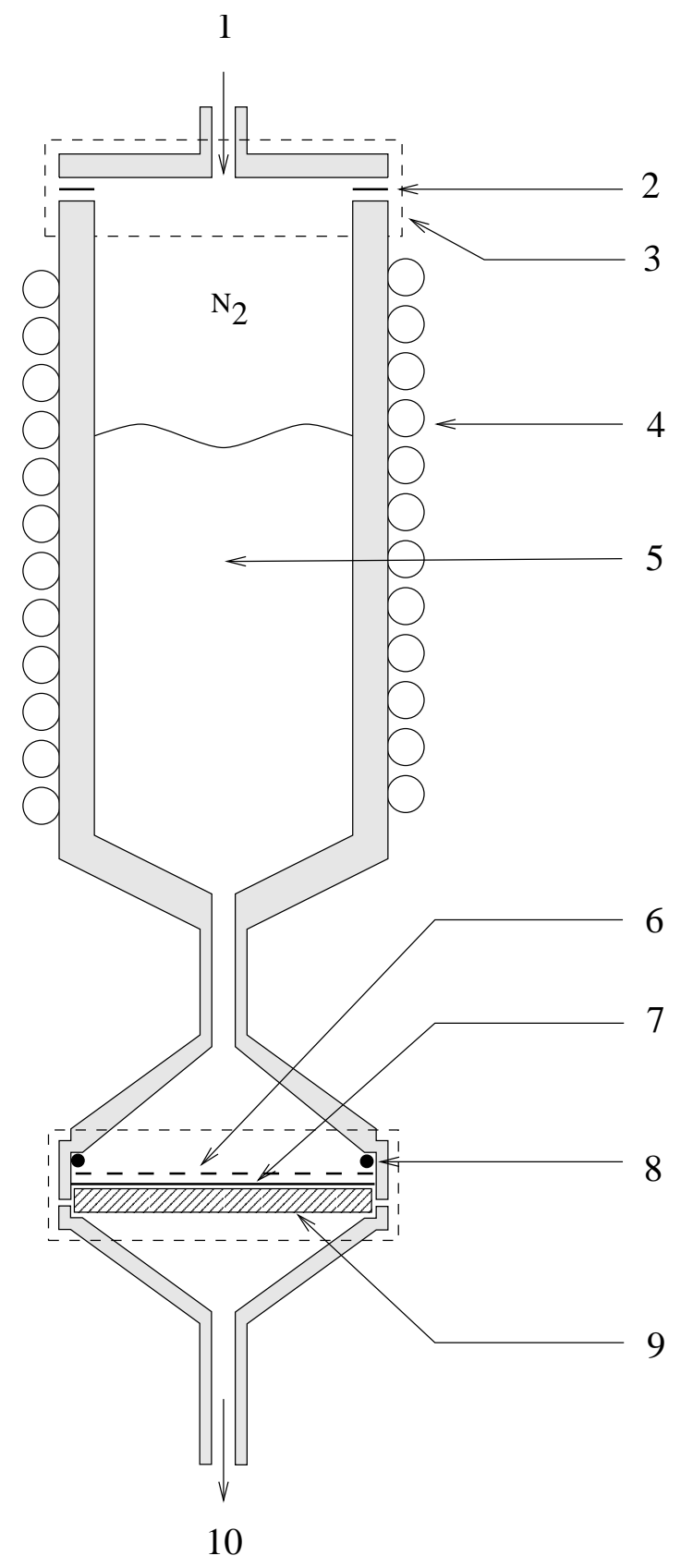

(1) Stickstoffzugang vom Reduzierventil

(2) Teflondichtung

(3) Überwurfmutter

(4) Thermostatierkanäle

(5) Probevolumen (ca. $100 \mathrm{ml}$ )

(6) Drain disc, zum Schutz der Membran

(7) $100 \mathrm{~nm}$ Polycarbonatmembran

(8) Viton-Ring

(9) Edelstahlgaze (ca. $30 \mu \mathrm{m}$ Maschenweite) als Membrantäger

(10) Ausgang zum Kolben

Abb. 2.8: Querschnitt durch den großen Extruder 


\subsubsection{Der große Extruder}

Abb. 2.8 zeigt den in der Institutswerkstatt gebauten großen Extruder. Er besitzt ein Fassungsvermögen von ca. $140 \mathrm{ml}$. Mit ihm werden die Vesikelsuspensionen, welche zur Messung der Ultraschallabsorptionsspektren verwendet werden, präpariert. Probe und Extruder werden vor und während der Extrusion mit einem Wasserbad temperiert, damit gewährleistet ist, daß der Extrusionsvorgang über der Hauptumwandlung der Lipide stattfindet. Die Suspensionen werden unter einem Druck von ca. $4 \frac{\mathrm{kp}}{\mathrm{cm}^{2}}$ durch die in Tabelle 2.1 aufgeführten Filter gedrückt. Die Drain Discs dienen dabei zum Schutz der eigentlichen Membranfilter, da diese bei dem herrschenden Druck und der auftretenden mechanischen Beanspruchung sonst leicht reißen können.

\subsubsection{Der LiposoFast-Basic Extruder}

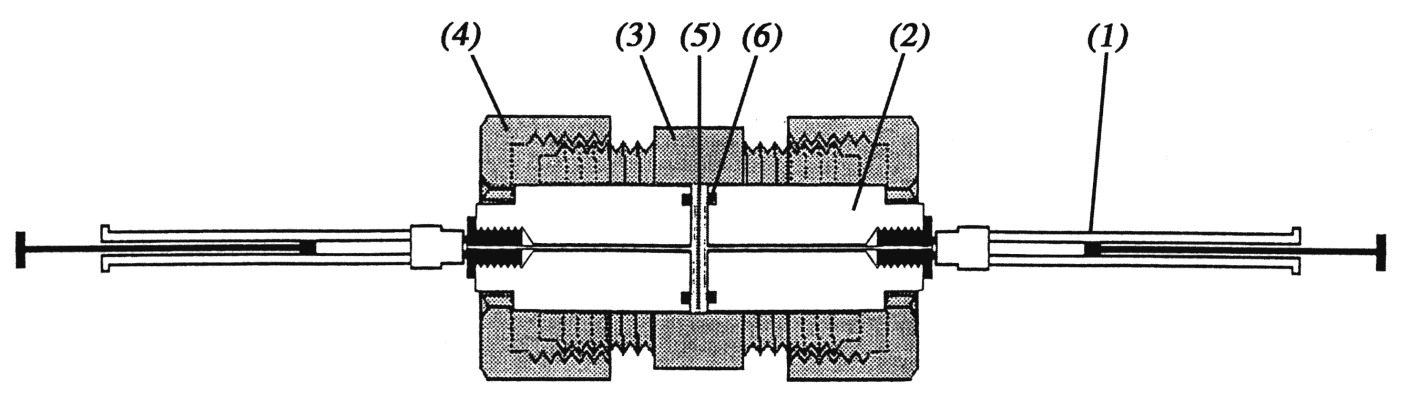

Abb. 2.9: Querschnitt durch LiposoFast-Basic Extruder, (1) Hamiltion-Spritze, (2) Hartplastikstopfen, (3) Metallgehäuse, (4) Überwurfmutter, (5) Membran, (6) Ringdichtung

Alle Suspensionen für die Kalorimetrie, Schallgeschwindigkeitsmessung und dielektrische Spektroskopie werden mittels des kommerziellen, kleineren Extruders in Abb. 2.9 präpariert. Zwei $1 \mathrm{ml}$ große Spritzen sind gegenüberliegend angebracht. Zwischen ihnen befindet sich der Filter, durch den mittels manuellem Durchdrücken der Spritzen, die Suspension extrudiert wird. Leider können, bedingt durch die einfache Konstruktion, weder Temperatur noch Druck definiert eingestellt werden. Jedoch wurde angestrebt, die Temperatur der Suspension durch Erwärmen mit Hilfe eines handelsüblichen Föhns bzw. eines Wasserbades, die Suspension wenigstens über der Umwandlungstemperatur während der Extrusion zu halten.

\begin{tabular}{|l|l|l|}
\hline $\begin{array}{l}\text { Membrane Filters, } \\
\text { Polycarbonate Membranes }\end{array}$ & $0.1 \mu \mathrm{m}, 47 \mathrm{~mm}$ & Catalog No. 13010 \\
\hline Polyester Drain Discs & $47 \mathrm{~mm}, 50 / \mathrm{pk}$ & Catalog No. 87487 \\
\hline \hline $\begin{array}{l}\text { Membrane Filters, } \\
\text { Polycarbonate Membranes }\end{array}$ & $0.1 \mu \mathrm{m}, 19 \mathrm{~mm}$ & \\
\hline
\end{tabular}

Tabelle 2.1: Die im Extruder verwendeten Filterkomponenten 


\subsubsection{Der Durchmesser der präparierten Vesikel}

Die spezielle Präparation des Extrudierens erzeugt eine Suspension unilamellarer Vesikel, deren Größe einer gewissen Verteilung unterliegt. Mittels quasielastischer Lichtstreuung [70] [149] [5] [89] [64] konnte exemplarisch an einer DMPC Suspension, die mit Hilfe des LiposoFast-Basic Extruders präpariert wurde, der mittlere Durchmesser bestimmt werden. Die entsprechende Verteilung des Radius bei Verwendung der in Tabelle 2.1 aufgeführten Membranen ist in Abb. 2.10 dargestellt.

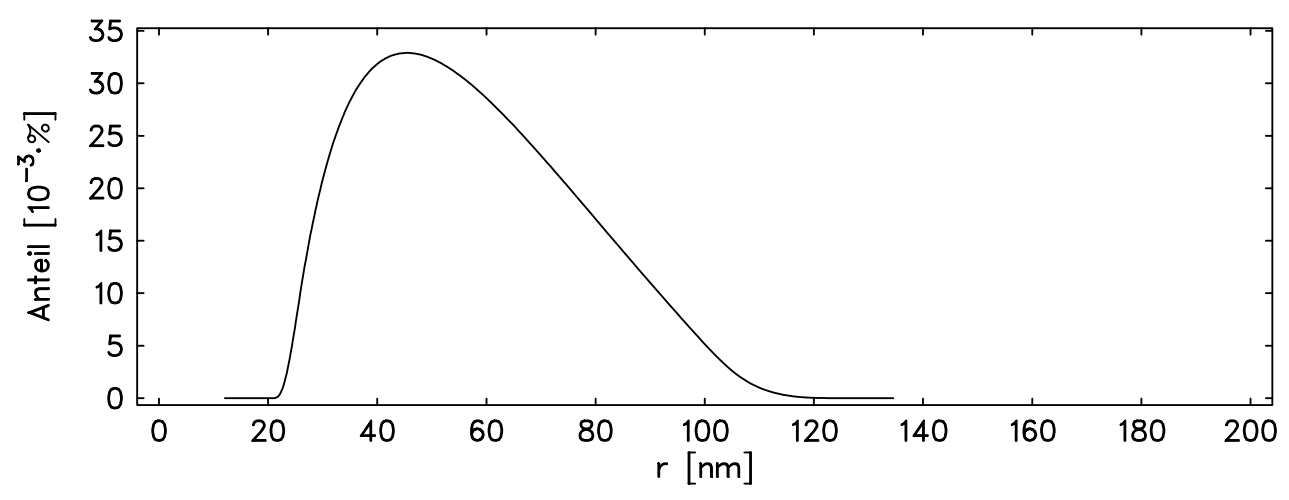

Abb. 2.10: Größenverteilung der Radien einer mit dem LiposoFastBasic Extruder präparierten Vesikelsuspension

\subsection{Zusammensetzung der verwendeten Suspensio- nen}

\subsubsection{Hersteller und Reinheit}

Alle verwendeten Substanzen wurden synthetisch hergestellt und wurden kommerziell erworben. Die Eigenschaften und die Reinheitsgrade der Substanzen verschiedener Hersteller (Fluka Chemie AG, CH-9471 Buchs; Sigma-Aldrich Chemie GmbH P.O. 1120, 89552 Steinheim) unterscheiden sich nicht wesentlich voneinander, so daß hier durchaus Lipide unterschiedlicher Hersteller miteinander gemischt werden konnten, z.B. bei den DMPC/DMG Suspensionen. Die einzelnen Spezifikationen sind in Tabelle 2.2 aufgeführt.

\begin{tabular}{|c|c|c|c|c|}
\hline Hersteller & Name & Chem. Formel & Molmasse & Reinheit \\
\hline \hline Fluka & DLPC & $C_{32} \mathrm{H}_{64} \mathrm{NO}_{8} \mathrm{P} \cdot \mathrm{H}_{2} \mathrm{O}$ & 637,8 & $>99 \%$ \\
Fluka & DMPC & $\mathrm{C}_{36} \mathrm{H}_{72} \mathrm{NO}_{8} \mathrm{P} \cdot \mathrm{H}_{2} \mathrm{O}$ & 695,96 & $>99 \%$ \\
Fluka & DPPC & $C_{40} \mathrm{H}_{80} \mathrm{NO}_{8} \mathrm{P} \cdot \mathrm{H}_{2} \mathrm{O}$ & 752,07 & $\approx 99 \%$ \\
Sigma & DMG & $\mathrm{C}_{31} \mathrm{H}_{60} \mathrm{O}_{5}$ & 512,80 & $\approx 99 \%$ \\
Fluka & Chol & $\mathrm{C}_{27} \mathrm{H}_{46} \mathrm{O}$ & 386,67 & $>99 \%$ \\
\hline
\end{tabular}

Tabelle 2.2: Herstellerangaben zur Reinheit der Lipide 


\subsubsection{Fehlerbetrachtungen}

Zur Messung der Ultraschallspektren werden jeweils $150 \mathrm{ml}$ angesetzt, für alle anderen Experimente $10 \mathrm{ml}$. Das Gewicht von $150 \mathrm{ml}$ bzw. $10 \mathrm{ml}$ Wasser wird mit einer elektronischen Waage bestimmt, deren maximaler systematischer Fehler bei $\pm 1,0$ mg liegt. Die jeweilige Menge an Lipid wird auf einer Präzisionswaage abgewogen, die einen Fehler von $\pm 0,1 \mathrm{mg}$ besitzt. Dementsprechend können die Konzentrationen der Suspensionen relativ genau abgewogen werden. Die sich daraus ergebenden Fehler in der Konzentration für die Lösungen der Ultraschallspektroskopie liegen bei etwa $0,1 \%$, während der Fehler der Konzentration der Lösungen für die anderen Experimente mit ca. $1 \%$ anzugeben sind.

Einer besonderen Betrachtung bedürfen die Lösungen für die dielektrischen Messungen mit extrem hoher Konzentration. Der Extrusionsvorgang gestaltete sich, verursacht durch den hohen Lipidanteil, extrem schwierig. Insbesondere bei der DMPC/DMG und DMPC/DPPC Suspension mußte der Filter mehrmals wegen Reißens gewechselt werden. Deshalb wird der Fehler in der Konzentration hier mit ca. $5 \%$ angegeben.

\subsubsection{Lösungen in der Kalorimetrie}

Für die kalorimetrischen Experimente werden jeweils "nur" 10 ml benötigt. Üblicherweise werden dabei relativ geringe Konzentrationen verwendet. Die Messungen der DLPC/DPPC Mischungen wurden von M. Kahle durchgeführt [85] und durch einen Rechenfehler wurden die Suspensionen mit $50 \mathrm{mg} / \mathrm{ml}$ angesetzt. Die verwendeten Lösungen sind in Tabelle 2.3 aufgeführt.

\begin{tabular}{|c|c|c||c|c|c||c|c|c|}
\hline $\begin{array}{c}\text { DMPC } \\
{[m o l \%]}\end{array}$ & $\begin{array}{c}\text { DPPC } \\
{[m o l \%]}\end{array}$ & $\begin{array}{c}\mathrm{c} \\
{\left[\frac{\mathrm{mg}}{\mathrm{ml}}\right]}\end{array}$ & $\begin{array}{c}\text { DMPC } \\
{[\mathrm{mol} \%]}\end{array}$ & $\begin{array}{c}\text { DMG } \\
{[\mathrm{mol} \%]}\end{array}$ & $\begin{array}{c}\mathrm{c} \\
{\left[\frac{\mathrm{mg}}{\mathrm{ml}}\right]}\end{array}$ & $\begin{array}{c}\text { DLPC } \\
{[\mathrm{mol} \%]}\end{array}$ & $\begin{array}{c}\text { DPPC } \\
{[\mathrm{mol} \%]}\end{array}$ & $\begin{array}{c}\mathrm{c} \\
{\left[\frac{\mathrm{mg}}{\mathrm{ml}}\right]}\end{array}$ \\
\hline 100 & 0 & 2 & 100 & 0 & 4 & 100 & 0 & 50 \\
83 & 17 & 2 & 95 & 5 & 4 & 80 & 20 & 50 \\
50 & 50 & 2 & 75 & 25 & 4 & 60 & 40 & 50 \\
17 & 83 & 2 & 60 & 40 & 4 & 40 & 60 & 50 \\
0 & 100 & 2 & 50 & 50 & 4 & 20 & 80 & 50 \\
& & & & & & 0 & 100 & 50 \\
\hline
\end{tabular}

Tabelle 2.3: Konzentration c und Zusammensetzung der in der Kalorimetrie verwendeten Suspensionen

\subsubsection{Lösungen der Schallgeschwindigkeitsmessungen}

Ähnlich wie in der Kalorimetrie, wird in der Schallgeschwindigkeitsmessung nur wenig Volumen für die Messungen benötigt. Die DMPC/DMG Messungen wurden sogar vom gleichen Ansatz, wie er auch in der Kalorimetrie verwendet wurde, durchgeführt. Alle verwendeten Lösungen sind in Tabelle 2.4 aufgeführt. 


\begin{tabular}{|c|c|c|c|c|c|c|c|c|}
\hline $\begin{array}{l}\mathrm{DMPC} \\
{[m o l \%]}\end{array}$ & $\begin{array}{l}\text { DPPC } \\
{[m o l \%]}\end{array}$ & {$\left[\begin{array}{c}\mathrm{c} \\
{\left[\frac{m g}{m l}\right]}\end{array}\right.$} & $\begin{array}{l}\text { DMPC } \\
{[m o l \%]}\end{array}$ & $\begin{array}{c}\text { DMG } \\
{[\mathrm{mol} \%]}\end{array}$ & {$\left[\begin{array}{c}\mathrm{c} \\
{\left[\frac{m g}{m l}\right]}\end{array}\right.$} & $\begin{array}{l}\text { DLPC } \\
{[\text { mol\%] }}\end{array}$ & $\begin{array}{l}\text { DPPC } \\
{[\text { mol\%] }}\end{array}$ & $\begin{array}{c}\mathrm{C} \\
{\left[\frac{m g}{m l}\right]}\end{array}$ \\
\hline 100 & 0 & 4 & 100 & 0 & 4 & 60 & 40 & 5 \\
\hline 83 & 17 & 4 & 95 & 5 & 4 & 40 & 60 & 5 \\
\hline 50 & 50 & 4 & 75 & 25 & 4 & 20 & 80 & 5 \\
\hline 17 & 83 & 4 & 60 & 40 & 4 & 0 & 100 & 4 \\
\hline 0 & 100 & 4 & 50 & 50 & 4 & & & \\
\hline & $\begin{array}{l}\text { DMPC } \\
{[\text { mol\%] }}\end{array}$ & $\begin{array}{c}\text { DLPC } \\
{[m o l \%]}\end{array}$ & {$\left[\begin{array}{c}\mathrm{c} \\
{\left[\frac{m g}{m l}\right]}\end{array}\right.$} & $\begin{array}{l}\text { DMPC } \\
{[\text { mol\%] }}\end{array}$ & \multicolumn{2}{|c|}{$\begin{array}{c}\text { Cholesterin } \\
\text { [mol\%] }\end{array}$} & $\begin{array}{c}\mathrm{C} \\
{\left[\frac{m g}{m l}\right]}\end{array}$ & \\
\hline & 100 & 0 & 4 & 95 & \multicolumn{2}{|r|}{5} & 5 & \\
\hline & 88 & 12 & 4 & 92,7 & \multicolumn{2}{|r|}{7,5} & 5 & \\
\hline & 76 & 24 & 4 & 90 & \multicolumn{2}{|r|}{10} & 5 & \\
\hline & 64 & 36 & 4 & 85 & \multicolumn{2}{|r|}{15} & 4 & \\
\hline & 52 & 48 & 4 & 80 & \multicolumn{2}{|r|}{20} & 4 & \\
\hline & 40 & \multirow[t]{2}{*}{60} & 4 & 66 & \multicolumn{2}{|r|}{33} & 4 & \\
\hline & & & & 50 & \multicolumn{2}{|r|}{50} & 4 & \\
\hline
\end{tabular}

Tabelle 2.4: Konzentration und Zusammensetzung der in den Schallgeschwindigkeitsmessungen verwendeten Suspensionen

\subsubsection{Lösungen in der dielektrikschen Spektroskopie}

Die Experimente in der dielektrischen Spektroskopie sind sensitiv für die Kopfgruppenumlagerung der Lipide. Da dieser Effekt jedoch vergleichsweise schwach ausgeprägt ist, müssen für diese Messungen entsprechend hohe Konzentrationen angesetzt werden. In einer konzentrationsabhängigen Meßreihe konnte der gesuchte Effekt deutlich sichtbar gemacht werden, so daß alle folgenden Suspensionen mit 60 $\mathrm{mg} / \mathrm{ml}$ angesetzt wurden. Alle verwendeten Lösungen sind in Tabelle 2.5 aufgeführt.

\begin{tabular}{|c|c|c||c|c|c||c|c|c|}
\hline $\begin{array}{c}\text { DMPC } \\
{[\mathrm{mol} \%]}\end{array}$ & $\begin{array}{c}\text { DPPC } \\
{[\mathrm{mol} \%]}\end{array}$ & $\begin{array}{c}\mathrm{c} \\
{\left[\frac{\mathrm{mg}}{\mathrm{ml}}\right]}\end{array}$ & $\begin{array}{c}\text { DMPC } \\
{[\mathrm{mol} \%]}\end{array}$ & $\begin{array}{c}\text { Cholesterin } \\
{[\mathrm{mol} \%]}\end{array}$ & $\begin{array}{c}\mathrm{c} \\
{\left[\frac{\mathrm{mg}}{\mathrm{ml}}\right]}\end{array}$ & $\begin{array}{c}\text { DMPC } \\
{[\mathrm{mol} \%]}\end{array}$ & $\begin{array}{c}\text { DMG } \\
{[\mathrm{mol} \%]}\end{array}$ & $\begin{array}{c}\mathrm{c} \\
{\left[\frac{\mathrm{mg}}{\mathrm{ml}}\right]}\end{array}$ \\
\hline 100 & 0 & 60 & 100 & 0 & 60 & 100 & 0 & 60 \\
50 & 50 & 60 & 85 & 15 & 60 & 75 & 25 & 60 \\
& & & 70 & 30 & 60 & & & \\
\hline
\end{tabular}

Tabelle 2.5: Konzentration und Zusammensetzung der in der dielektrischen Spektroskopie verwendeten Suspensionen

\subsubsection{Lösungen in der Ultraschallspektroskopie}

Für die Messungen der Ultraschallspektren werden jeweils ca. $150 \mathrm{ml}$ benötigt. Wegen dieser vergleichsweise großen Menge werden die Lösungen größtenteils mit 2 $\mathrm{mg} / \mathrm{ml}$, einige jedoch auch mit $10 \mathrm{mg} / \mathrm{ml}$, angesetzt. Da die Ultraschallabsorptionsspektroskopie sensitiv gegenüber Volumenänderungen innerhalb der Membran ist, und dieser Effekt relativ stark ausgeprägt ist, kann auch mit den $2 \mathrm{mg} / \mathrm{ml}$ Lösungen 
gut gearbeitet werden. Alle in der Ultraschallspektroskopie verwendeten Lösungen sind in Tabelle 2.6 aufgeführt.

DMPC

\begin{tabular}{|c|c|c|c|c|c|c|c|c|c|c|c|c|c|c|}
\hline $\mathrm{c}\left[\frac{m g}{m l}\right]$ & 10 & 2 & 10 & 2 & 10 & 10 & 2 & 10 & 2 & 2 & 2 & 10 & 2 & 10 \\
\hline $\mathrm{T}\left[{ }^{\circ} \mathrm{C}\right]$ & 15 & 18 & 20 & 22 & 23,5 & 24 & 24 & 24,5 & 25 & 26 & 27 & 28 & 30 & 32 \\
\hline & & \multicolumn{2}{|c|}{$\begin{array}{l}\text { DMPC } \\
{[\text { mol } \%]}\end{array}$} & \multicolumn{3}{|c|}{$\begin{array}{c}\text { Cholesterin } \\
\text { [mol\%] }\end{array}$} & $\begin{array}{c}\mathrm{C} \\
{\left[\frac{m g}{m l}\right]}\end{array}$ & \multicolumn{4}{|c|}{$\begin{array}{c}\text { gemessene } \mathrm{T} \\
\mathrm{T}\left[{ }^{\circ} \mathrm{C}\right]\end{array}$} & & & \\
\hline & & \multicolumn{2}{|c|}{95} & \multicolumn{3}{|c|}{5} & 10 & \multicolumn{4}{|c|}{$20,24,28$} & & & \\
\hline & & \multicolumn{2}{|c|}{92,5} & \multicolumn{3}{|c|}{7,5} & 10 & \multicolumn{4}{|c|}{$20,24,28$} & & & \\
\hline & & \multicolumn{2}{|c|}{90} & \multicolumn{3}{|c|}{10} & 10 & \multicolumn{4}{|c|}{$20,24,28$} & & & \\
\hline & & & 85 & \multicolumn{3}{|c|}{15} & 2 & \multicolumn{4}{|c|}{$20,23,24,25,28$} & & & \\
\hline & & & 66 & \multicolumn{3}{|c|}{33} & 2 & \multicolumn{4}{|c|}{$20,23,24,25,28$} & & & \\
\hline & & \multicolumn{2}{|c|}{$\begin{array}{l}\text { DMPC } \\
{[\mathrm{mol} \%]}\end{array}$} & \multicolumn{3}{|c|}{$\begin{array}{l}\mathrm{DPPC} \\
{[\mathrm{mol} \%]}\end{array}$} & $\begin{array}{c}\mathrm{C} \\
{\left[\frac{m g}{m l}\right]}\end{array}$ & \multicolumn{4}{|c|}{$\begin{array}{c}\text { gemessene } \mathrm{T} \\
\mathrm{T}\left[{ }^{\circ} \mathrm{C}\right]\end{array}$} & & & \\
\hline & & \multicolumn{2}{|c|}{83} & \multicolumn{3}{|c|}{17} & 2 & \multicolumn{4}{|c|}{$23,26,27,28,31$} & & & \\
\hline & & \multirow{2}{*}{\multicolumn{2}{|c|}{$\begin{array}{l}50 \\
17\end{array}$}} & \multicolumn{3}{|c|}{50} & 2 & \multicolumn{4}{|c|}{$29,32,33,34,37$} & & & \\
\hline & & & & \multicolumn{3}{|c|}{83} & 2 & \multicolumn{4}{|c|}{$34,37,38,39,42$} & & & \\
\hline
\end{tabular}

Tabelle 2.6: Konzentration und Zusammensetzung der in der Ultraschallspektroskopie verwendeten Suspensionen 


\section{Schallgeschwindigkeitsmessung/ Kalorimetrie}

Das Phasenverhalten der untersuchten Suspensionen, diverse Systemparameter und deren Temperaturabhängigkeit ist für die Charakterisierung der Suspensionen von besonderem Interesse. Mit Hilfe eines DSC (Differential Scanning Caloriemeters) [133] [139] und eines Schallgeschwindigkeitsresonators ist die Möglichkeit dafür gegeben, die Wärmekapazität und die Schallgeschwindigkeit in Abhängigkeit von der Temperatur zu messen. Außerdem sind über das Fluktuations-Dissipations-Theorem die Wärmekapazität $C_{p}$ und die isotherme Volumenkompressibilität $\kappa_{T}$ direkt mit der Fluktuation von Enthalpie und Volumen verknüpft (siehe Gleichung (3.10)).

Von der Seite der Molekularakustik bieten Schallgeschwindigkeitsmessungen bei fester Frequenz eine zur Ultraschallabsorptionsspektroskopie ergänzende Herangehensweise [60] [59] [106] [147]. Die Schallgeschwindigkeitsmessung ermöglicht mit vergleichsweise geringem Aufwand eine hohe Temperaturauflösung und benötigt nur sehr wenig Volumen (ca. $3 \mathrm{ml}$ ).

Die Apparaturen zur Wärmekapazitäts- und Schallgeschwindigkeitsmessung zeichnen sich durch äußerst hohe Präzision aus, was zu sehr genauen Meßergebnissen führt. Es sollen nun die beiden Meßverfahren kurz vorgestellt werden und im weiteren wird eine Verknüpfung beider Daten mittels einer Theorie von T. Heimburg [53] [49] angestrebt.

\subsection{Kalorimetrie - Meßverfahren}

Da dem 3. Physikalischen Institut kein Kalorimeter zur Verfügung steht, wurden alle Messungen der Wärmekapazität in enger Zusammenarbeit mit der Arbeitsgruppe Thermodynamik von Membranen, Dr. T. Heimburg am MPI für Biophysikalische Chemie Göttingen durchgeführt.

In der Kalorimetrie wird einer Probe bei konstantem Druck eine bestimmte Wärmemenge $\delta Q$ zugeführt / abgeführt und die darauf resultierende Änderung der Temperatur $\delta T$ gemessen. Die Wärmekapazität $C_{p}$ bei konstantem Druck ergibt sich dann als

$$
C_{p}(T)=\left(\frac{\delta Q}{\delta T}\right)_{p} .
$$

Die Umwandlungsenthalpie ergibt sich entsprechend zu

$$
\Delta H=\int_{T_{1}}^{T_{2}} C_{p}(T) d T .
$$

Da beide Größen nur für den thermodynamischen Gleichgewichtszustand definiert sind, muß die Messung der spezifischen Wärme sehr langsam erfolgen. Ein typischer "Scan" über einen Temperaturbereich von ca. $50^{\circ} \mathrm{C}$ nimmt daher etwa 24 Stunden in Anspruch.

Kalorimetrie ist hier als differentielles Verfahren realisiert. Die Temperaturänderung, die die Zufuhr / Abfuhr von Wärme in einer Meßzelle bewirkt, wird relativ zu der Temperaturänderung, die die gleiche Wärmemenge in der anderen Meßzelle 
verursachte, gemessen. Die so ermittelten Daten für die Meßprobe sind demzufolge Exzeßwerte gegenüber der Referenzsubstanz. Ändert die Probe während des Scans ihren Phasenzustand, wie es bei den untersuchten Lipidsuspensionen der Fall war, so äußert sich dies in einem charakteristischem "Peak" in der $C_{p}$-Kurve.

In einem Differentialkalorimeter befinden sich Referenz und Meßsubstanz in zwei benachbarten Behältern. Beide werden mit derselben Rate beheizt bzw. gekühlt. Findet nun in der einen Zelle ein Umwandlung statt, die zusätzlich gegenüber der Referenz Wärme benötigt, so wird diese Energie durch ein zusätzliches Thermoelement bereitgestellt. Die mittlere elektrische Leistung $\overline{P^{e x}}$, die für den Ausgleich der Temperatur nötig ist, wird als Meßgröße aufgenommen. Mit der daraus resultierenden Scan-Rate (sr), die ein Maß für die Geschwindigkeit ist, mit der das Experiment durchgeführt wird, ergibt sich die Exzeßwärmekapazität:

$$
\overline{P^{e x}} \cdot(s r)^{-1}=\overline{C_{p}^{e x}}
$$

\subsubsection{Aufbau des Differentialkalorimeters}

Die vorliegenden Daten wurden mit Hilfe eines kommerziellen Micro-Kalorimeters der Firma MicroCal Inc., Northampton, MA (USA) gewonnen. Der Fehler in der Temperaturbestimmung liegt bei $\Delta T= \pm 0,2 \mathrm{~K}$. In dem Kalorimeter befinden sich zwei fest eingebaute Behälter für Meß- und Referenzsubstanz.

\subsection{Kalorimetrie - Ergebnisse}

Wie oben schon erwähnt, ist es nötig, die kalorimetrische Messung möglichst langsam durchzuführen, damit sich das System im thermodynamischen Gleichgewicht befindet. Die Messungen wurden alle mit einer Scan-Rate $\mathrm{sr}=5 \mathrm{~K} / \mathrm{h}$ durchgeführt, was eine ausreichend langsame Heizrate darstellt. Um sicher zu gehen wurde zusätzlich exemplarisch eine Messung einer DMPC Suspension auch mit einer Scan-Rate $\mathrm{sr}=1 \mathrm{~K} / \mathrm{h}$ gemessen. Es zeigten sich jedoch keine signifikanten Unterschiede $\mathrm{zu}$ den Ergebnissen mit $\mathrm{sr}=5 \mathrm{~K} / \mathrm{h}$. Vor jeder Messung wurde jede Probe eine Stunde lang bei der jeweiligen Starttemperatur im Kalorimeter temperiert.

Die Kurven der DMPC/DPPC-Mischungen sind von M. Höckel [65], die DMPC/DMG-Mischungen von F. Wente [148] und die DLPC/DPPC-Mischungen von M. Kahle [85] entnommen.

In den Abb. 3.1 bis Abb. 3.4 sind nun die Wärmekapazitätsmessungen aufgetragen. Bei allen Suspensionen zeigt sich ein jeweils charakteristisches Maximum in $\operatorname{der} C_{p^{-}}$ Kurve, das auf einen Schmelzprozeß der Ketten in der Lipiddoppelschicht schließen läßt.

Abbildung 3.1 zeigt ferner, daß sich die Gestalt der Wärmekapazitätskurven der DMPC/DPPC-Suspensionen mit zunehmenden DPPC Anteil deutlich verändern. Zeigt reines DMPC noch einen relativ deutlich ausgeprägten Doppelpeak, so verbreitert sich die Wärmekapazitätskurve mit wachsendem DPPC Anteil. Die Umwandlungstemperatur steigt deutlich an, bis bei reinem DPPC ein relativ scharfer Peak bei ca. $42{ }^{\circ} \mathrm{C}$ zu sehen ist. Besonders hervorzuheben ist an dieser Stelle, daß trotz der sehr unterschiedlichen Umwandlungstemperaturen der beiden beteiligten Lipide, sieht man von dem relativ schwach ausgeprägtem Doppelpeak bei DMPC 


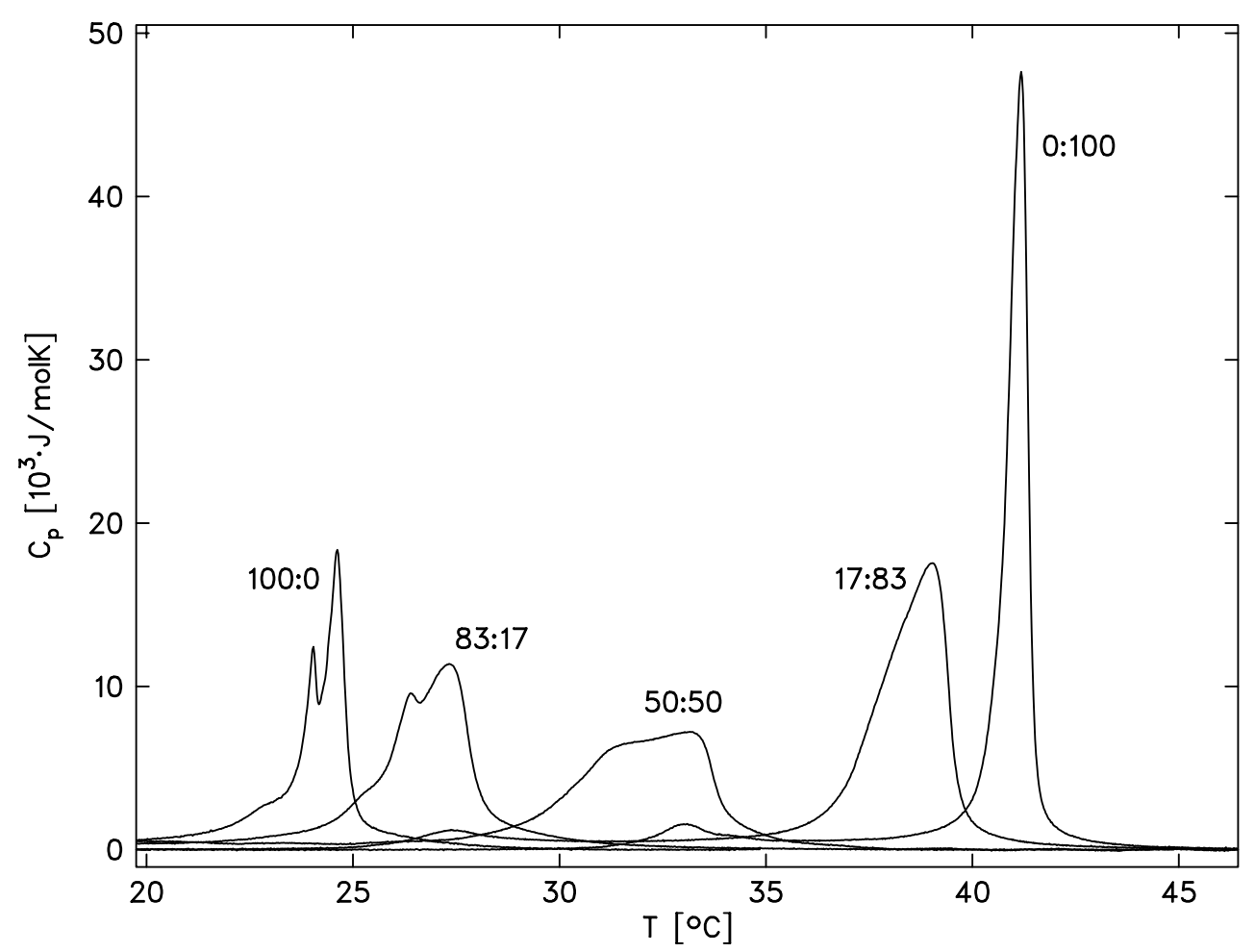

Abb. 3.1: DMPC/DPPC-Mischungen $2 \mathrm{mg} / \mathrm{ml}, \mathrm{sr}=5 \mathrm{~K} / \mathrm{h}$, upscan, der Anteil an DPPC nimmt von links nach rechts zu (Messungen von M. Höckel [65]).

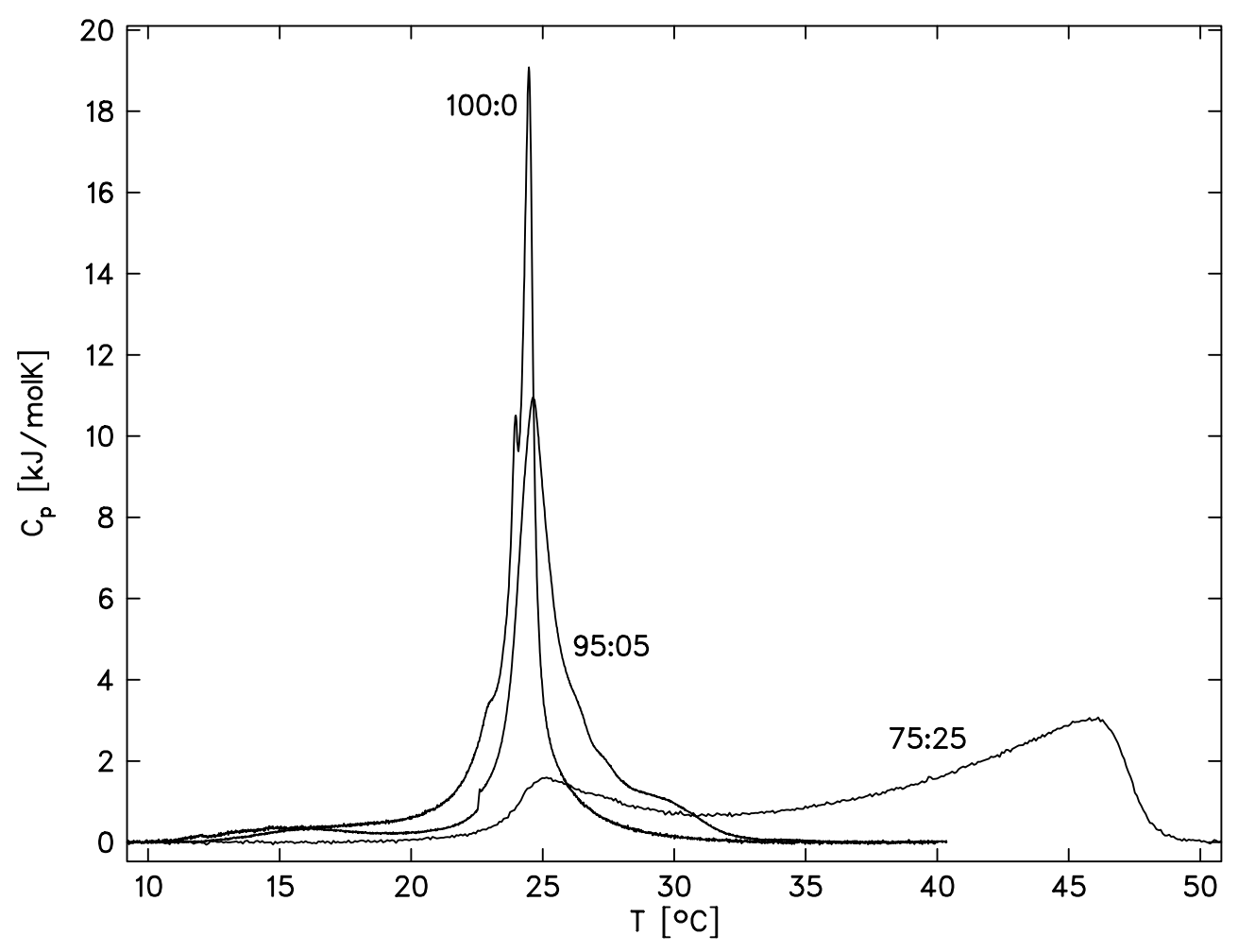

Abb. 3.2: DMPC/DMG-Mischungen $2 \mathrm{mg} / \mathrm{ml}$, sr=5 K/h, upscan, der Anteil an DMG nimmt von links nach rechts zu (Messungen von F. Wente [148]). 


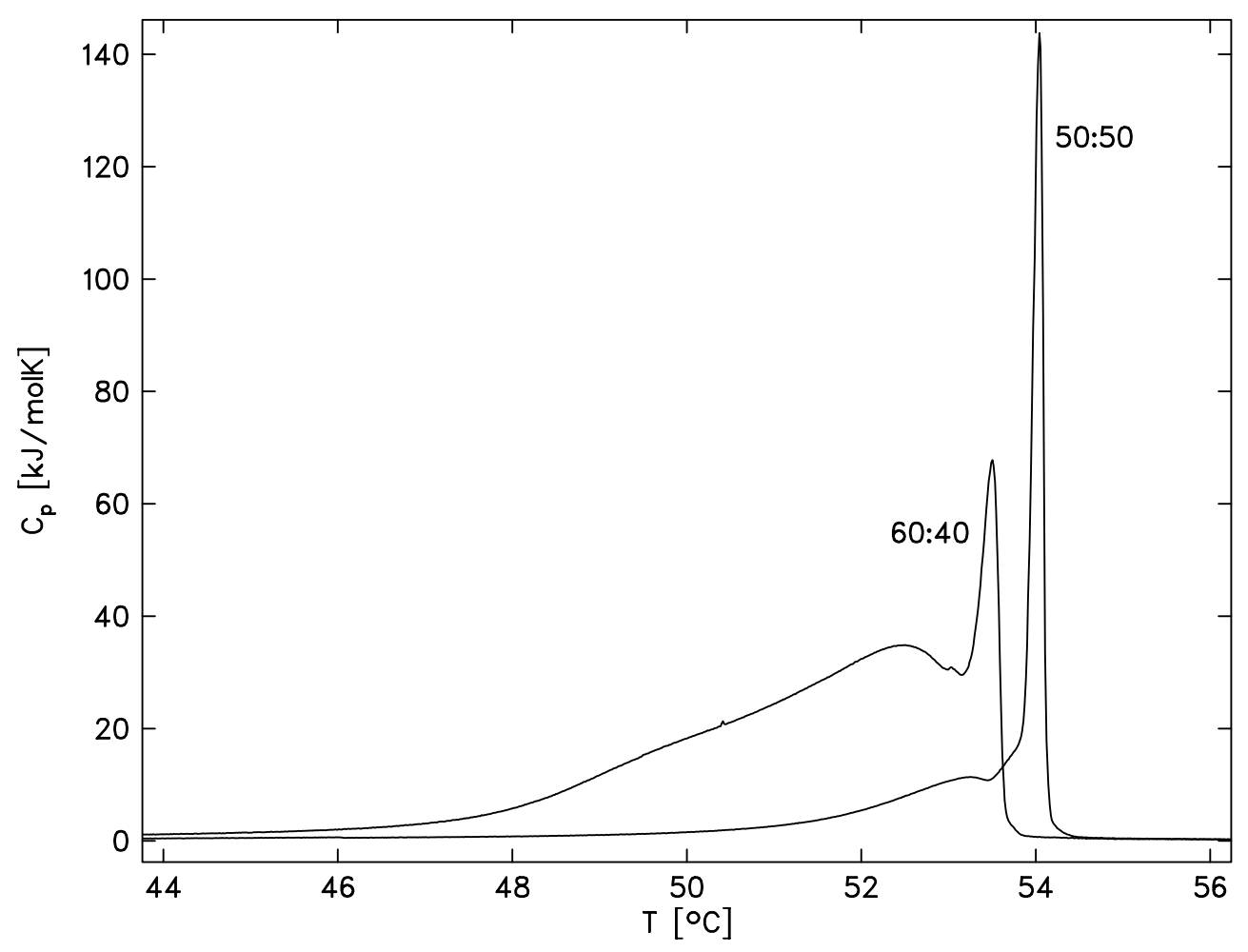

Abb. 3.3: DMPC/DMG-Mischungen $2 \mathrm{mg} / \mathrm{ml}, \mathrm{sr}=5 \mathrm{~K} / \mathrm{h}$, upscan, der Anteil an DMG nimmt von links nach rechts zu (Messungen von F. Wente [148]).

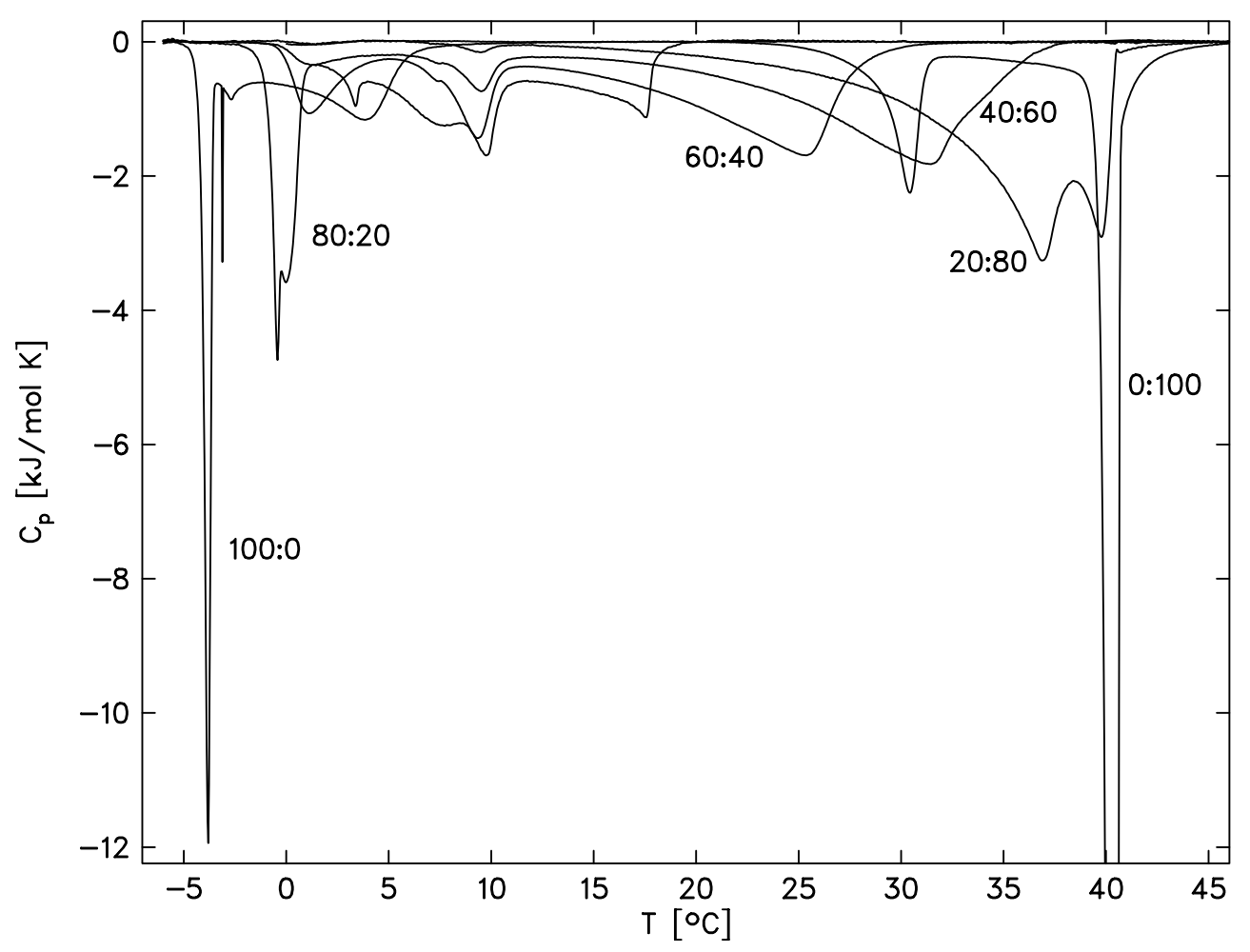

Abb. 3.4: DLPC/DPPC-Mischungen (multilammellare Vesikel) 50 $\mathrm{mg} / \mathrm{ml}, \mathrm{sr}=5 \mathrm{~K} / \mathrm{h}$, downscan, der Anteil an DPPC nimmt von links nach rechts zu (Messungen von M. Kahle [85]). 
ab, sich immer nur ein Umwandlungspeak finden läßt, der den Schmelzvorgang der Alkylketten charakterisiert.

Ein von den DMPC/DPPC Mischungen gänzlich anderes Verhalten zeigen die DMPC/DMG Mischungen. In Abb. 3.2 und Abb. 3.3 sind die gemessenen Wärmekapazitätskurven der DMPC/DMG-Mischungen dargestellt. Während bei relativ kleinem DMG Anteil sich die Umwandlungstemperatur nur sehr wenig von DMPC unterscheidet, ergibt sich bei der 75:25 Mischung eine sehr markante Verbreiterung der Umwandlung. Außerdem lassen sich zwei verschiedene, weit auseinander liegende Umwandlungstemperaturen ausmachen. Steigt der DMG Anteil noch weiter, so verschiebt sich die Umwandlungstemperatur mehr und mehr zu $54{ }^{\circ} \mathrm{C}$. Hier muß beachtet werden, daß keine Mischungen mit eine höherem DMG Gehalt als 50 \% gemessen wurden, im Gegensatz zu den DMPC/DPPC Mischungen, wo Mischungen bis zu reinem DPPC betrachtet wurden. Insbesondere bei den Mischungen mit hohem DMG Anteil gab es erhebliche Konzentrationsprobleme, die sich schon durch große Extrusionsschwierigkeiten äußerten. Während der Messung der Wärmekapazität im Kalorimeter ballten sich zusätzlich die aufgelösten Lipidmoleküle zu einer einzigen großen, kugelförmigen Struktur zusammen, was sicherlich für die fehlerhafte Exzeß-Enthalpie in Tabelle 3.1 verantwortlich ist.

\begin{tabular}{|c|c|c|c|c|c|c|c|c|c|}
\hline $\mathrm{DMPC} / \mathrm{DPPC}$ & $\begin{array}{c}T_{1} \\
{\left[{ }^{\circ} \mathrm{C}\right]}\end{array}$ & $\begin{array}{c}T_{2} \\
{\left[{ }^{\circ} \mathrm{C}\right]}\end{array}$ & $\begin{array}{l}\Delta H^{e z} \\
{\left[\frac{k J}{m o l}\right]}\end{array}$ & \multicolumn{3}{|c|}{ DMPC/DMG } & $\begin{array}{c}T_{1} \\
{\left[{ }^{\circ} \mathrm{C}\right]}\end{array}$ & $\begin{array}{r}T_{2} \\
{\left[{ }^{\circ} \mathrm{C}\right]}\end{array}$ & $\begin{array}{c}\Delta H^{e x} \\
{\left[\frac{k J}{m o l}\right]}\end{array}$ \\
\hline 100:00 & 12 & 30 & 26,4 & \multicolumn{3}{|c|}{ 100:00 } & 12 & 40 & 28,4 \\
\hline $83: 17$ & 12 & 38 & 33,2 & \multicolumn{3}{|c|}{$95: 05$} & 10 & 40 & 30,3 \\
\hline $50: 50$ & 17 & 38 & 33,8 & \multicolumn{3}{|c|}{$75: 25$} & 15 & 50 & 36 \\
\hline $17: 83$ & 23 & 46 & 43,9 & \multicolumn{3}{|c|}{$60: 40$} & 30 & 60 & (153) \\
\hline 00:100 & 31 & 46 & 41,4 & \multicolumn{3}{|c|}{$50: 50$} & 30 & 60 & 53 \\
\hline & \multicolumn{3}{|c|}{ DLPC/DPPC } & $\begin{array}{c}T_{1} \\
{\left[{ }^{\circ} \mathrm{C}\right]}\end{array}$ & $\begin{array}{c}T_{2} \\
{\left[{ }^{\circ} \mathrm{C}\right]}\end{array}$ & \multicolumn{2}{|c|}{$\begin{array}{l}\Delta H^{e x} \\
\left\lceil\frac{k J}{m o l}\right\rceil\end{array}$} & & \\
\hline & \multicolumn{3}{|c|}{ 100:00 } & -6 & 50 & 19,6 & & & \\
\hline & \multicolumn{3}{|c|}{$80: 20$} & -6 & 50 & 27,4 & & & \\
\hline & \multicolumn{3}{|c|}{$60: 40$} & -6 & 50 & 32,8 & & & \\
\hline & \multicolumn{3}{|c|}{ 40:60 } & -6 & 50 & 33,1 & & & \\
\hline & \multicolumn{3}{|c|}{$20: 80$} & -6 & 50 & 41,7 & & & \\
\hline & \multicolumn{3}{|c|}{ 00:100 } & -6 & 50 & 41,4 & & & \\
\hline
\end{tabular}

Tabelle 3.1: Den Wärmekapazitätskurven bei Integration von $T_{1}$ bis $T_{2}$ entnommene Exzeß-Enthalpien

Bei den DLPC/DPPC Suspensionen handelt es sich im Gegensatz zu den anderen obigen Kurven um "downscans" bei denen die Temperatur von einem hohen Startwert ausgehend langsam erniedrigt wird. Die Wärmekapazitätskurven der DLPC/DPPC Mischungen (Abb. 3.4) zeigen im Vergleich zu den DMPC/DPPC Suspensionen ebenso ein deutlich unterschiedliches Verhalten. Es ist bei den ausgeglichenen Mischungsverhältnissen der beiden Komponenten DLPC und DPPC ein relativ komplexes Schmelzverhalten im Gegensatz zu den DMPC/DPPC Mischungen zu sehen. Insbesondere lassen sich ähnlich wie bei den DMPC/DMG Messungen deutlich zwei, bei DLPC/DPPC 80:20 sogar drei verschiedene Umwandlungspeaks 
ausmachen.

Auf kalorimetrische Messungen von DMPC/Cholesterin Suspensionen wurde hier verzichtet, da ein ausreichender Datensatz früherer Messungen vorliegt [49] [38].

Aus den gewonnenen $C_{p}$-Daten läßt sich mittels Gleichung (3.2) die Exzeß-Enthalpie berechnen. Es wird dafür jeweils der volle Integrationsweg von $T_{1}$ bis $T_{2}$ (der gesamte verfügbare Scan der Wärmekapazität) verwendet. Die so gewonnenen ExzeßEnthalpien sind in Tabelle 3.1 aufgeführt.

\subsection{Schallgeschwindigkeitsmessung - Meßverfahren}

\subsubsection{Aufbau der Schallgeschwindigkeitsapparatur}

Das Kernstück der Apparatur zur Messung der Schallgeschwindigkeit [97] besteht aus zwei, in einem einzigen Metallblock eingearbeiteten, zylinderförmigen Hohlraumresonatoren. Beide Resonatoren werden an ihren Ende durch zwei elektroakustische Quarzwandler (biplanar) mit einer Grundresonanz von $5 \mathrm{MHz}$ abgeschlossen. Zur prinzipiellen Funktionsweite eines flüssigkeitsgefüllten Hohlraumresonators siehe Kap. 6.1.

Der Doppelresonator ermöglicht es, gleichzeitig eine Meßprobe und eine Referenzflüssigkeit zu messen. Durch die spezielle Anordnung befinden sich beide Resonatoren im thermischen Gleichgewicht. Darüber hinaus herrschen zur jeder Zeit möglichst gleiche Bedingungen in beiden Hohlräumen. Somit liegen idealer Weise die sich bildenden Resonanzen der beider Resonatoren bei gleichen Frequenzen. Dieses ermöglicht durch direkten Vergleich der Differenz der beiden Resonanzfrequenzen bei sich ändernder Temperatur eine sehr genaue Bestimmung der Variation der Schallgeschwindigkeit und damit der Schallgeschwindigkeit selbst. Die Steuerung der kompletten Apparatur übernimmt ein einfacher Rechner (Intel 486) (Abb. 3.5). Abb. 3.5 zeigt schematisch den Signalverlauf und die einzelnen Steuerelemente des Doppelresonators. Das sinusförmige Signal der Frequenz $\nu$ wird von einem Synthetisiersender (DDS, 2a) bereitgestellt und mittels (2ar) und (2as) verstärkt. Das Signal wird nun an einen der Quarze gegeben und wandelt dort das elektrische Signal in ein akustisches um. Die so erzeugte Schallwelle wird mehrfach an den Wandlern reflektiert und bildet somit ein Stehwellenfeld. Es bilden sich für den Hohlraumresonator (6r, 6s) charakteristische Resonanzen aus, deren Resonanzfrequenz hier die eigentliche Meßgröße darstellt. Das am Empfangswandler abgegriffene Singal wird nun im Mischer (5ar, 5as) mit einem Signal der Frequenz $\nu+10 \mathrm{kHz}$, welches durch einen zweiten Signalgenerator (DDS, 2b) erzeugt wird, gemischt. Das Signal wird weiter durch einen schmalbandigen Verstärker (8ar, 8as) geleitet und gelangt schließlich zum analog/digital Wandler (9ar, 9as). Ebenfalls analog/digital gewandelt wird ein Referenzsignal, welches vom zweiten Synthetisiersender (DDS, 2b) nach Verstärkung (2bs, 2br) und Mischung (5bs, 5br) mit dem Meßsignal bereitgestellt wird. Eine spezielle Prozeßkarte (DSP, 10) speichert während einer Messung einer Resonanzkurve alle anfallenden Daten zwischen und übergibt sie danach dem Computer (11) zur weiteren Bearbeitung. Zur Kontrolle und Stabilität des Systems ist ein $50 \mathrm{MHz}$ Triggersignalgenerator (1) integriert.

Da die Schallgeschwindigkeit indirekt über die Lage einer Resonanzfrequenz ermittelt wird, kommen für diesen Zweck nur einige wenige, der sonst zahlreich vorhan- 


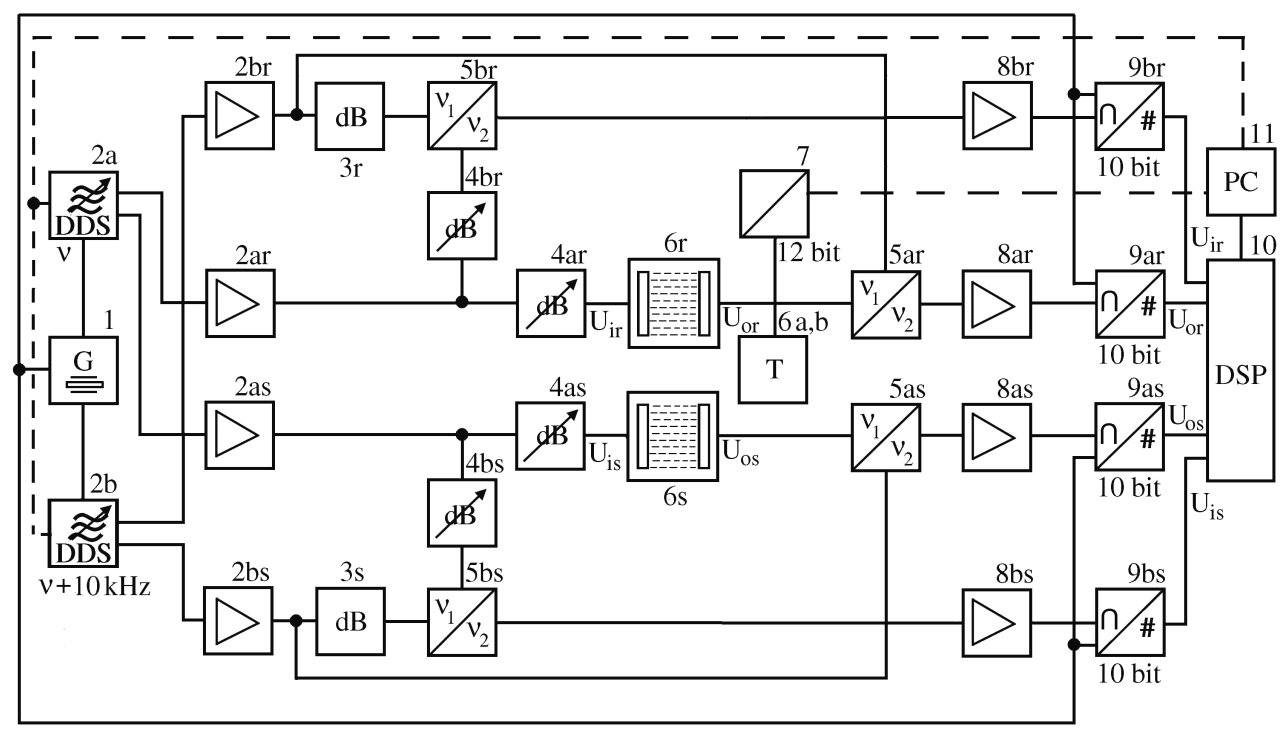

Abb. 3.5: Aufbau der Apparatur zur Messung der Schallgeschwindigkeit als Funktion der Temperatur; (s) Meßzweig, (r) Referenzzweig, (1) $50 \mathrm{MHz}$ Kontollsignalgenerator, (2a, 2b) Direkt Digital Synthetisierer $\nu=25 \mathrm{MHz},(3)$ festes Dämpfungsglied, (4a, 4b) variables Dämpfungsglied, (5a, 5b) Mischer, (6) Resonatordoppelzelle, (6a, 6b) Temperaturkontrolleinheit, (7) 12 bit D/A Wandler, (8) Verstärker $10 \mathrm{kHz}$, (9a, 9b) A/D Wandler, (10) DSP Prozeßkarte, (11) Computer

denen Resonanzen, in Frage. Im Bereich um 2,5 MHz besitzen die Resonanzen die geringste Halbwertsbreite und die Hauptmode wird nur unwesentlich durch Nebenmoden gestört, was die für diesen Zweck höchst mögliche Genauigkeit darstellt.

Für die hier geforderte extrem hohe Genauigkeit der gemessenen Schallgeschwindigkeit ist es nötig, die Temperatur im Resonator entsprechend genau einstellen zu können. Außerdem muß die Temperatur während der Messung konstant gehalten werden. Ferner muß sie mit entsprechender Genauigkeit bekannt sein. Deren Regelung erfolgt über Peltierelemente, um die Zelle zu kühlen oder zu erwärmen. Die Zelltemperatur wird über einen Halbleitertemperaturfühler beobachtet, welcher in Reihe mit einem Potentiometer geschaltet ist, um immer gleichen Stromfluß durch den Fühler zu gewährleisten. Die Spannung, die am Temperaturfühler anliegt, wird mittels eines 12 bit D/A Wandlers dem Computer zu Verfügung gestellt. Zusätzlich wird über einen Differenzverstärker ein entsprechendes Signal auf die Peltierelemente gegeben. Das Beobachten der Drift einer Resonanzfrequenz, überlicherweise in dem Resonator mit der Referenzflüssigkeit, stellt die genauste Methode zur Festlegung der Entscheidung, ob eine bestimmte Temperatur erreicht ist.

Für die Schallgeschwindigkeitsmessungen kann in guter Näherung davon ausgegangen werden, daß sich in den Zylinderresonatoren nur eine Mode in Längsrichtung ausbreitet. Alle auftretenden Nebenmoden können vernachlässigt werden. Wenn 1 die Länge des Resonators ist, gilt

$$
f_{n}=\frac{c \cdot n}{2 \cdot l}
$$

mit c der Schallgeschwindigkeit der Flüssigkeit und einer natürlichen Zahl n. Im Doppelresonator gilt dieses für beide Zellen: 


$$
l_{1}=n \cdot c_{1} \cdot \frac{1}{2 f_{1, n}} \quad, l_{2}=n \cdot c_{2} \cdot \frac{1}{2 f_{2, n}}
$$

Damit folgt weiter

$$
\left(l_{1}-l_{2}\right)=n \cdot\left(\frac{c_{1}}{2 f_{1, n}}-\frac{c_{2}}{2 f_{2, n}}\right)
$$

Es wird hier mit zwei verschiedenen Längen $l_{1}$ und $l_{2}$ gerechnet, da im realen Doppelresonator natürlich nicht beide Seiten vollständig baugleich sind. Dies wirkt sich u.a. darin aus, daß sich die Zellängen ein wenig unterscheiden. Die Zellängendifferenz $l_{\text {diff }}=\left(l_{1}-l_{2}\right)$ wurde in zahlreichen Referenzmessungen, bei denen sich in beiden Zellen Wasser befand, ausreichend genau bestimmt, so daß sie nun in die umgestellte Formel eingesetzt werden kann:

$$
c_{2}=2 f_{2} \cdot\left(\frac{c_{1}}{2 f_{1}}-\frac{l_{\text {diff }}}{n}\right) .
$$

Somit ergibt sich die Schallgeschwindigkeit der Suspension $c_{2}$ unter Zuhilfenahme der bekannten Schallgeschwindigkeit $c_{1}$ der Referenz, der bekannten Zellängendifferenz $l_{\text {diff }}$ und den gemessenen Resonanzfrequenzen $f_{1, n}$ und $f_{2, n}$ bei der entsprechenden Temperatur.

In den bisherigen Überlegungen gehen wir von einem idealen Resonator aus. In Wirklichkeit werden die sich im Resonator ausbreitenden Wellen nicht vollständig an der Grenzschicht zwischen Wandler und Lösung reflektiert. Das Resonanzverhalten der Quarze selbst führt zu einer Frequenzabhängigkeit der Impedanz der Wandler (siehe auch Kapitel 6.1). Somit ändert sich die effektive Länge des Resonators mit der Frequenz, was eine Verschiebung der Resonanzfrequenzen zur Folge hat. Daher ist es notwendig, die gemessenen Werte der Resonanzfrequenzen zu korrigieren. Eggers et al. geben in [24] dafür eine Beziehung an, die unter Verwendung des Impedanzverhältnisses von Wanlder zu Wasser $\frac{Z_{L}}{Z_{T}} \approx 0,1$ und der Quarzresonanz $f_{T_{m}}=5$ $\mathrm{MHz}$ die real gemessenen Resonanzfrequenzen $f_{n}$ darart korrigiert, daß man Werte für Resonanzfrequenzen $f_{L n}$ erhält, die sich auf die geometrische Zellänge bezieht:

$$
f_{L n}=f_{n} \cdot\left(n+\frac{2}{\pi} \cdot \arctan \left(\frac{Z_{L}}{Z_{T}} \cdot \cot \left(\pi \cdot \frac{f_{n}}{f_{T_{m}}}\right)\right)\right)^{-1} .
$$

Bei Lipidsuspensionen liegt es nahe, als Referenz Wasser zu verwenden, da sich die Schallgeschwindigkeit der Suspensionen nur sehr wenig von der des Wassers unterscheidet. Um diesen doch sehr geringen Unterschied gut zu verdeutlichen, führt man deshalb die Schallgeschwindigkeitszahl u ein:

$$
u=\frac{c_{\text {Meßprobe }}-c_{\text {Lösungsmittel }}}{c_{\text {Lösungsmittel }} \cdot k\left[\frac{m g}{m l}\right]}=\frac{c_{\text {Suspension }}-c_{\text {Wasser }}}{c_{\text {Wasser }} \cdot k\left[\frac{m g}{m l}\right]} .
$$

Eine ausführliche Beschreibung der Apparatur zur Messung der Schallgeschwindigkeit findet sich bei Lautscham et al. [97]. 


\subsubsection{Meßfehler}

Durch die spezielle Konstruktion der Doppelresonatormeßzelle ist es möglich, sehr genaue temperaturabhängige Schallgeschwindigkeiten zu messen. Durch zahlreiche Meßreihen mit reinem Wasser als Referenz, als auch durch wiederholtes Messen von Lipidsuspensionen konnte ein statistischer Fehler für die Bestimmung der Schallgeschwindigkeit und der Schallgeschwindigkeitszahl ermittelt werden.

- Für die geforderte hohe Genauigkeit müssen alle möglichen Fehlerquellen vermieden werden. Dabei spielt u.a. das Einfüllen der Flüssigkeiten in die Resonatoren eine entscheidene Rolle. Es besteht ein eklatanter Unterschied in der Genauigkeit der Ergebnisse, je nachdem ob die Flüssigkeit schnell oder sehr langsam in den Resonator gelangt. Bei schneller Befüllung wird nur eine relativ schlechte Benetzung der Quarzwandler erreicht, so daß im Verlauf der Messung die Veränderung der Benetzungseigenschaften sich in den Meßergebnissen widerspiegelt. Aus diesem Grund wird jede Befüllung mit einer speziellen Einfüllaparatur durchgeführt, bei der die mit der Meßflüssigkeit gefüllte Spritze mit definiert langsamer Geschwindigkeit in den Resonator entleert wird. Somit können Fehler dieser Art vermieden werden.

- Evident wichtig für die Genauigkeit der Apparatur ist die Stabilität der Temperatur. Die Stabilität der Temperatur ist bei der verwendeten Apparatur bis auf $0,001{ }^{\circ} \mathrm{C}$ genau, was den hier gestellten Ansprüchen genügt.

- Durch Wassermessungen und den Vergleich mit den Literaturdaten kann die Genauigkeit der Schallgeschwindigkeit mit $10^{-5} \%$ angegeben werden.

- Mittels wiederholter Messungen an einer DMPC-Lösung wurde der statistische Fehler der Schallgeschwindigkeitszahl bestimmt. Da die Schallgeschwindigkeitszahl sich oftmals um Null bewegt gebe ich hier einen absoluten Fehler anstelle eines relativen an. Er liegt bei $\pm 7 \cdot 10^{-6} \frac{\mathrm{ml}}{\mathrm{mg}}$ ).

\subsection{Schallgeschwindigkeitsmessung - Ergebnisse}

Um später direkte Vergleiche anstellen zu können, wurden für die Schallgeschwindigkeitsmessungen die gleichen Mischungsverhältnisse verwendet wie für die kalorimetrischen Messungen. Während die DMPC/DMG Suspensionen sogar bei beiden Meßverfahren dieselben waren, mußten aus organisatorischen Gründen bei den DMPC/DPPC Mischungen und bei den DLPC/DPPC Mischungen verschiedene Ansätze verwendet werden. Wie auch bei den Messungen der Wärmekapazität sollte bei der Messung der Schallgeschwindigkeit die Temperaturänderung möglichst langsam sein. Es hat sich gezeigt, daß eine Heizrate von ca. $3 \mathrm{~K} / \mathrm{h}$ einen guten Kompromiß darstellt. Vor jeder Messung wurde sowohl die Referenz als auch die Meßlösung gut entgast und dann mittels der Einfüllapparatur sehr langsam in den Doppelresonator eingefüllt. Alle Messungen der Temperaturprofile der Schallgeschwindigkeit erfolgten in $1 \mathrm{~K}$ Schritten und jeweils von tiefen Temperaturen über die zu erwartende Umwandlungstemperatur zu der jeweiligen höheren Endtemperatur. Um Vergleiche anstellen zu können, wurde auch eine Meßserie mit DMPC/Cholesterin 


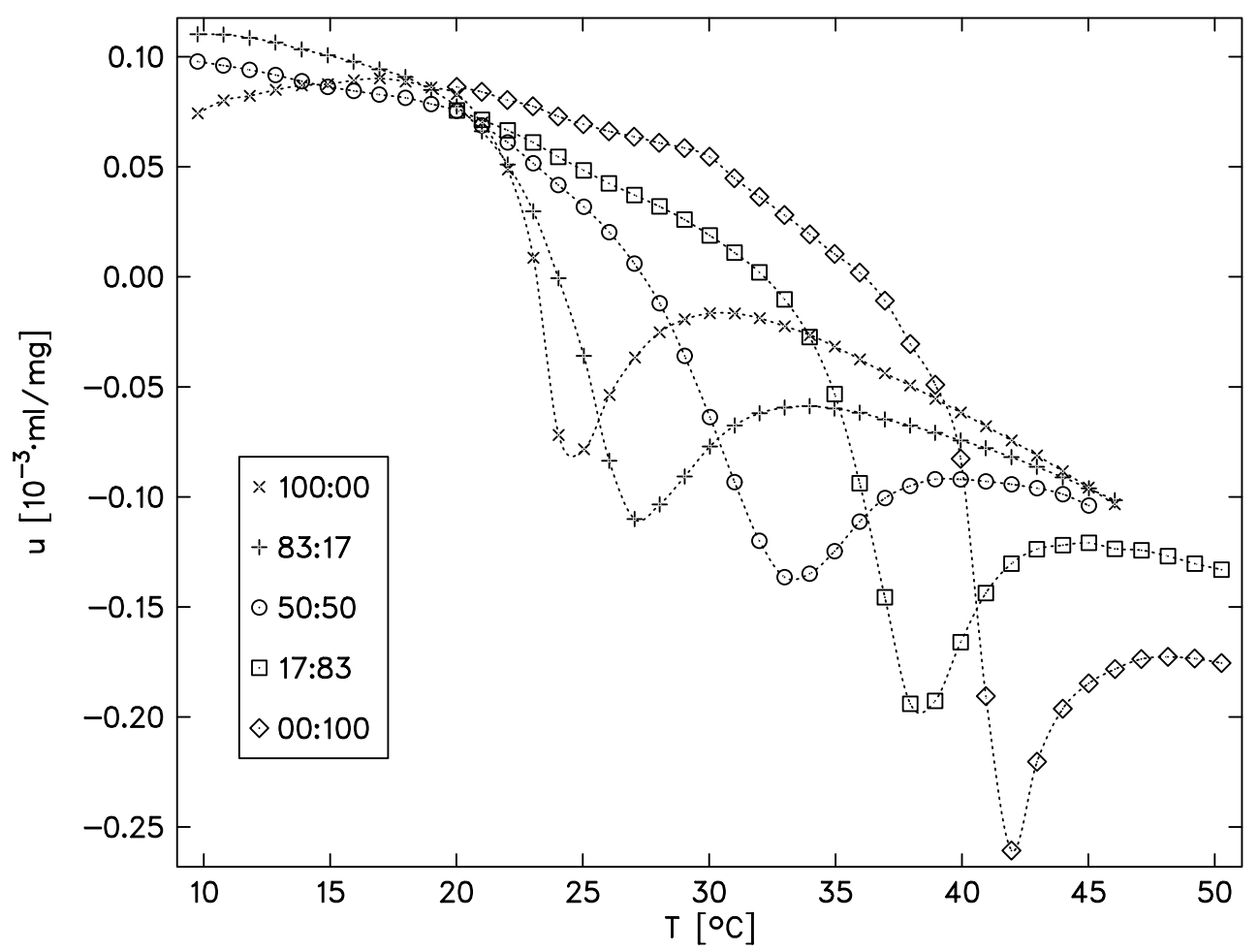

Abb. 3.6: Schallgeschwindigkeitszahlen der DMPC/DPPC Suspensionen als Funktion der Temperatur

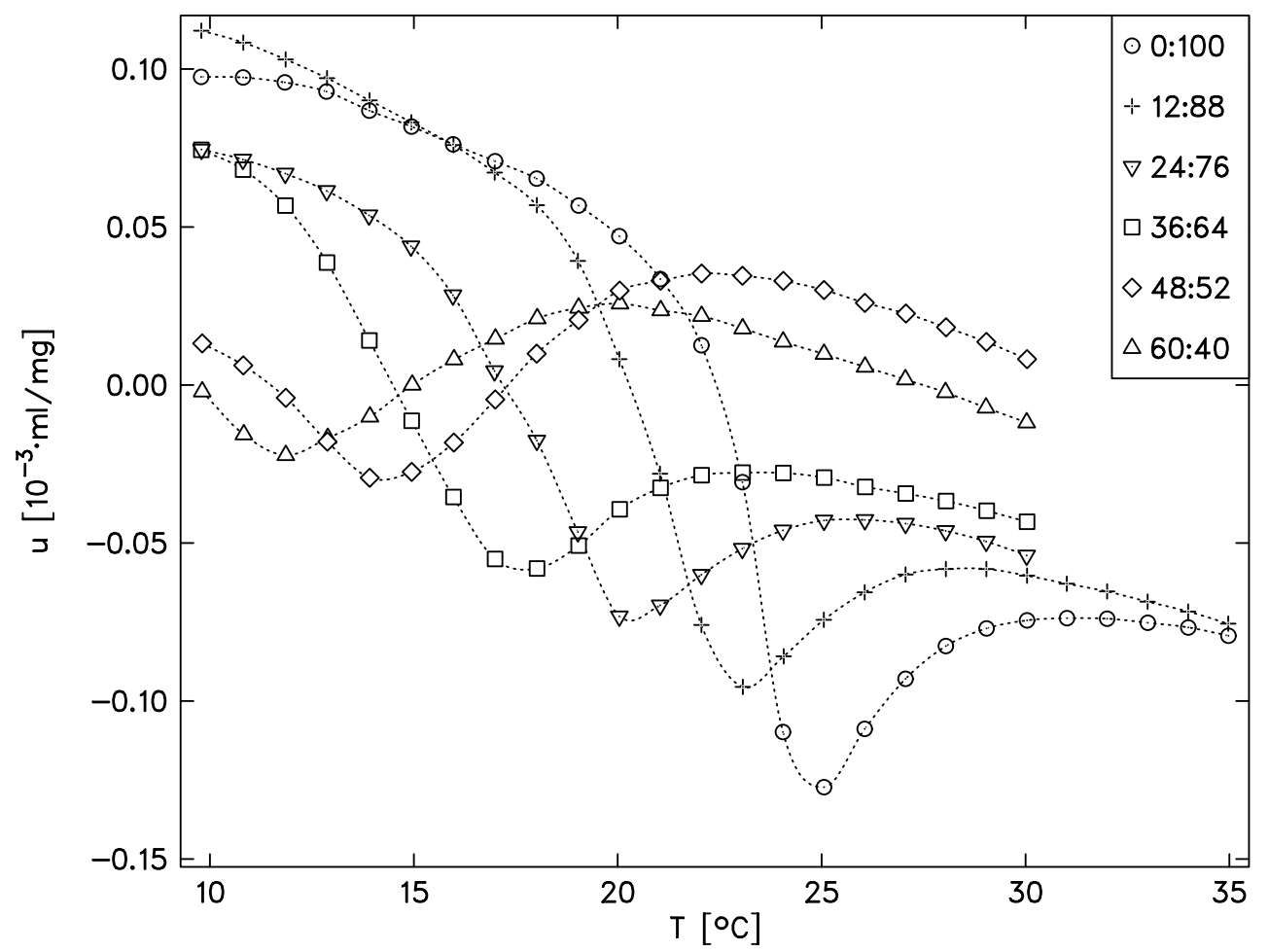

Abb. 3.7: Schallgeschwindigkeitszahlen der DMPC/DLPC Suspensionen als Funktion der Temperatur 


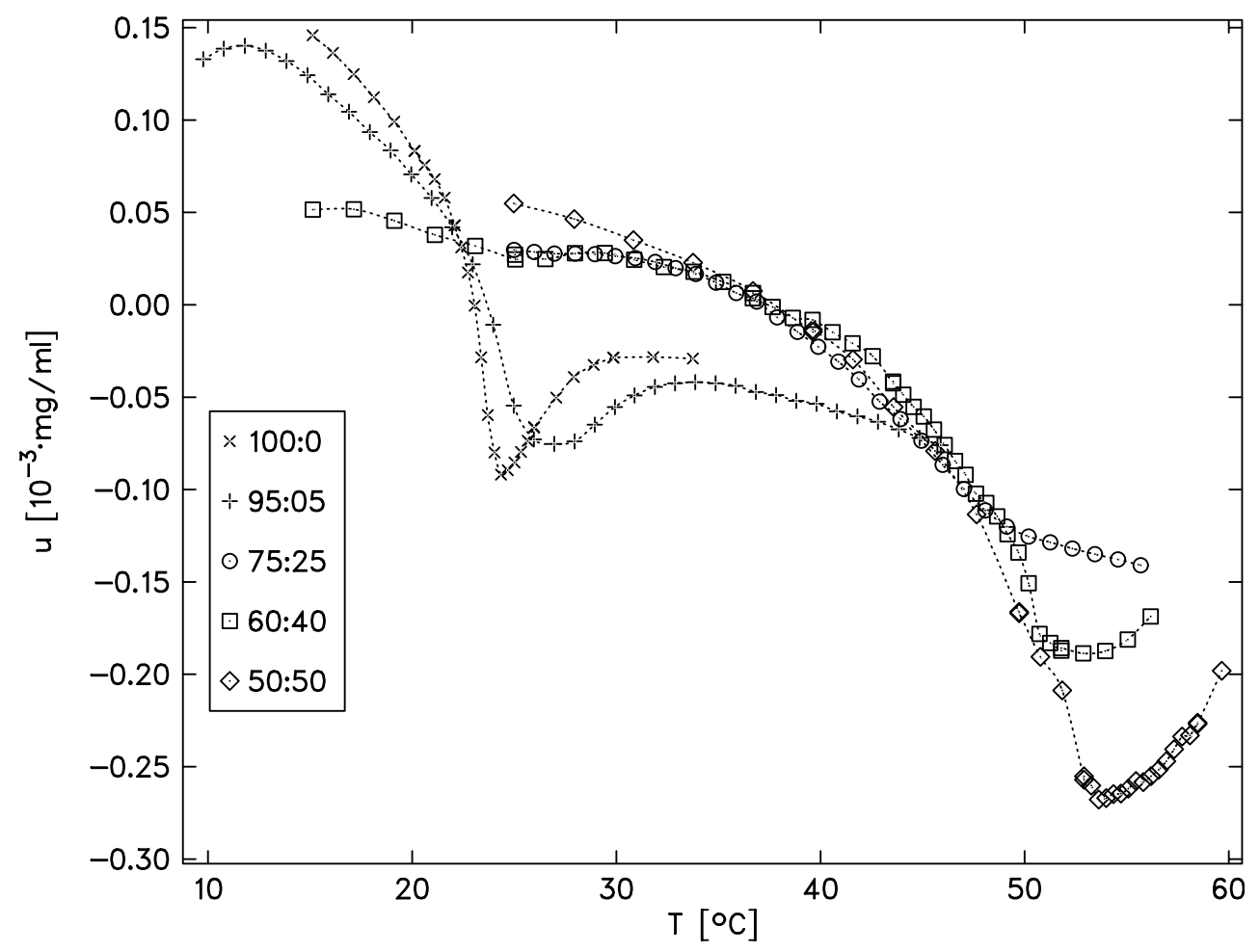

Abb. 3.8: Schallgeschwindigkeitszahlen der DMPC/DMG Suspensionen als Funktion der Temperatur (Messungen teilweise von F. Wente [148])

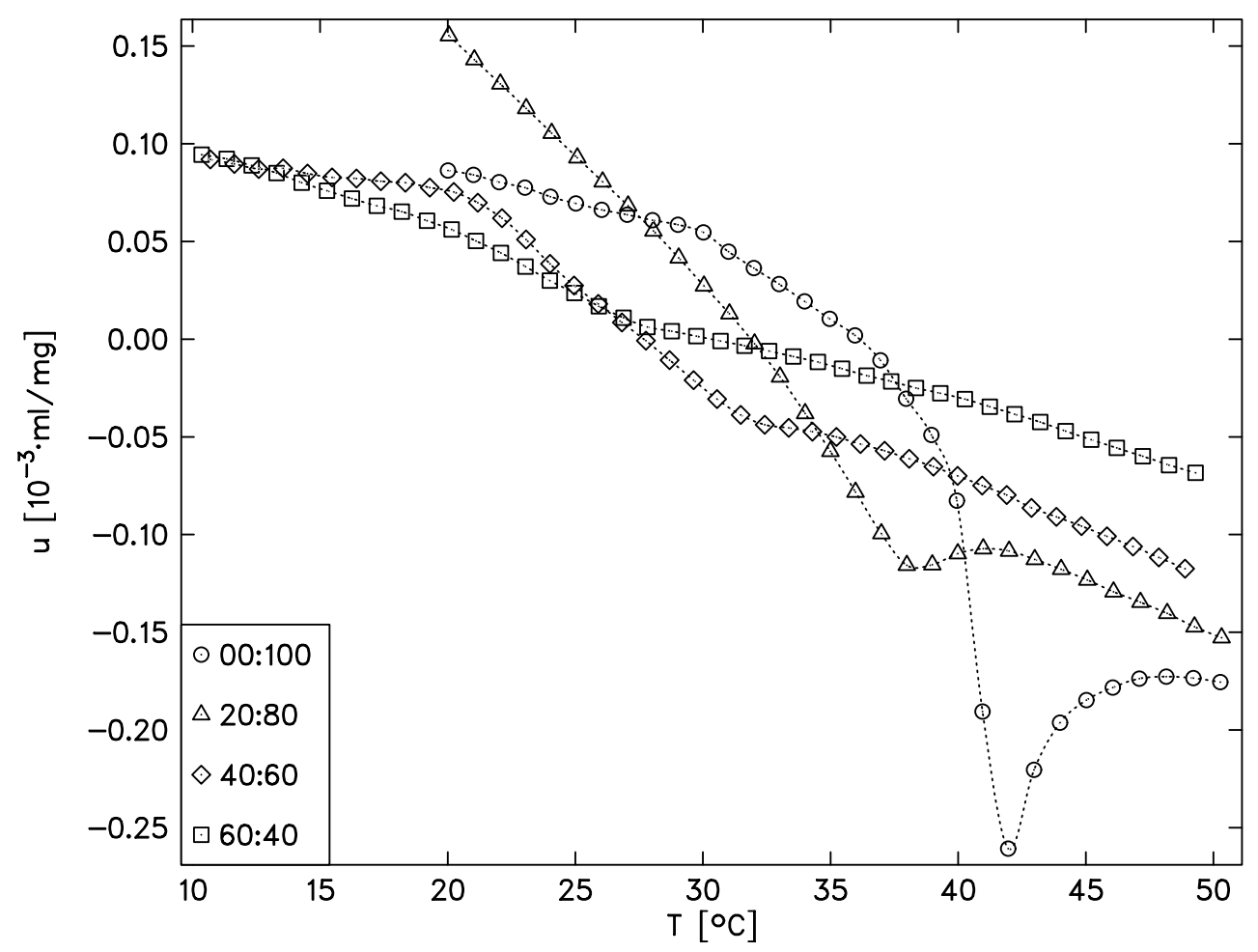

Abb. 3.9: Schallgeschwindigkeitszahlen der DLPC/DPPC Suspensionen als Funktion der Temperatur 


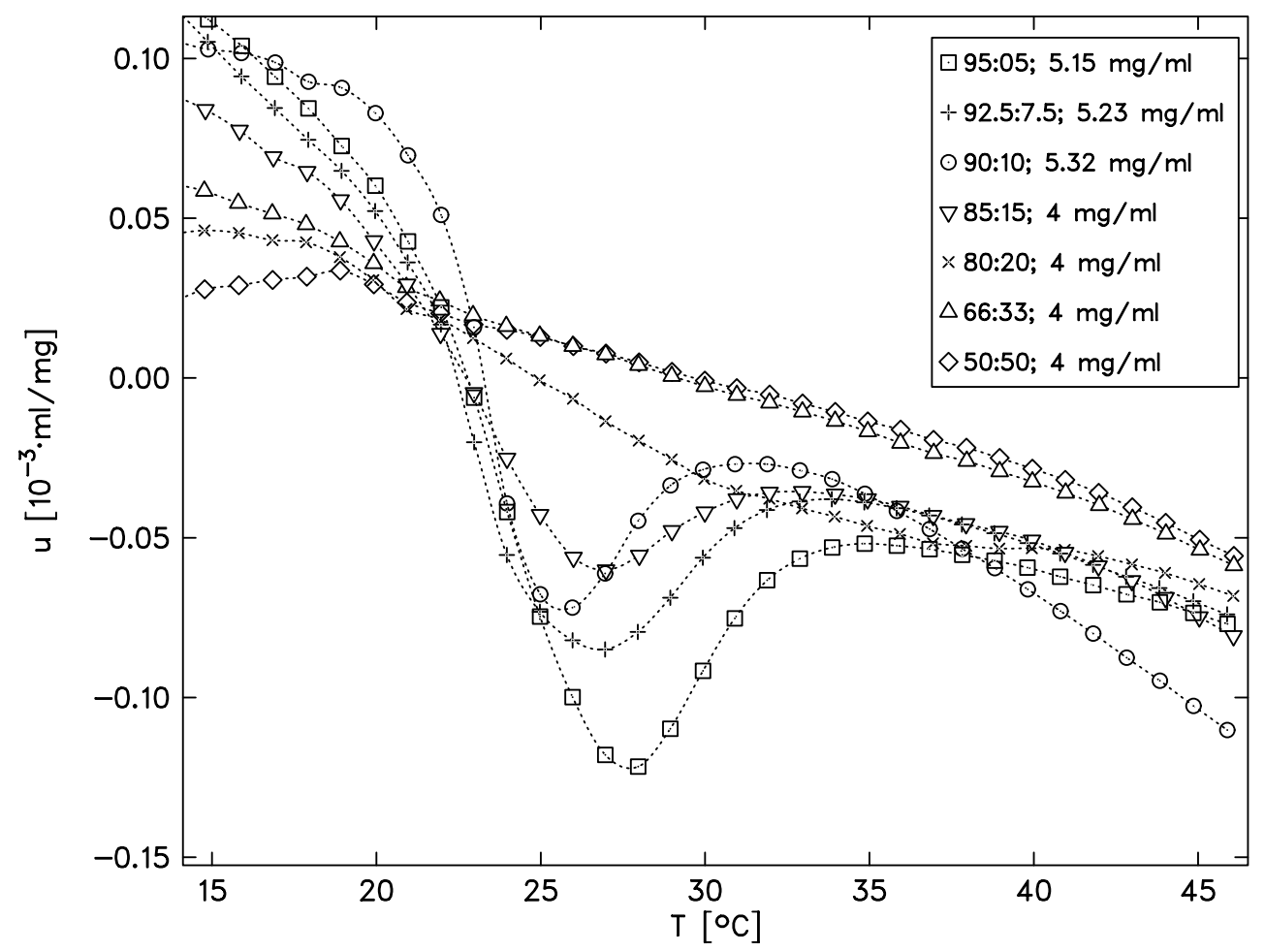

Abb. 3.10: Schallgeschwindigkeitszahlen der DMPC/Cholesterin Suspensionen als Funktion der Temperatur

Mischungen durchgeführt. Die Kurven der DMPC/DMG Suspensionen sind teilweise bei F. Wente [148] entnommen.

In Abb. 3.6 sind die Schallgeschwindigkeitszahlen der DMPC/DPPC Mischungen aufgetragen [106]. Wie auch bei den Wärmekapazitätskurven ist hier deutlich die Verschiebung der Umwandlungstemperatur mit steigendem DPPC Gehalt hin zu $42^{\circ} \mathrm{C}$ zu sehen [22]. Außerdem ist eine Verbreiterung der Umwandlung auszumachen, je ausgeglichener das Mischungsverhältnis ist. Der in den kalorimetrischen Messungen gefundene Doppelpeak bei reinem DMPC ist hier nicht zu finden.

Abb. 3.7 zeigt zu den DMPC/DPPC Messungen ergänzende Messungen von DLPC/DMPC Mischungen. Da DLPC eine entsprechend tiefere Schmelztemperatur als DMPC besitzt, ist zu erwarten, daß sich bei steigendem DLPC Gehalt die Umwandlungstemperatur erniedrigt, was auch in den Schallgeschwindigkeitsmessungen deutlich zu erkennen ist. Da die Schallgeschwindigkeitsapparatur nicht tiefer als $10{ }^{\circ} \mathrm{C}$ zu messen in der Lage ist, konnten die beiden Lösungen mit dem Mischungsverhältnis 52:48 und 40:60 nicht ausreichend genug zu tiefen Temperaturen hin ausgemessen werden.

Wie auch in den kalorimetrischen Messungen zu sehen, zeigt sich bei den Schallgeschwindigkeitskurven der DMPC/DMG Mischungen in Abb. 3.8 deutlich, daß die 75:25 Mischung zwei Umwandlungstemperaturen besitzt. Geringe Beimischung von DMG bewirkt eine leichte Verbreiterung der Umwandlung, während sich bei höherem DMG Gehalt die Umwandlung bei ca. $52^{\circ} \mathrm{C}$ stabilisiert.

Abb. 3.9 zeigt die Schallgeschwindigkeitszahlen der DLPC/DPPC Mischungen. Im Gegensatz zu den DMPC/DPPC bzw. DLPC/DMPC Mischungen ist hier eine nicht so starke Verschiebung der Umwandlungstemperatur mit der Änderung des Mi- 
schungsverhältnisses zu verzeichnen, wie es im Vergleich zur DMPC/DPPC Mischung zu erwarten wäre. Es bilden sich bei den ausgewogenen Mischungsverhältnissen eher zwei charakteristische Umwandlungstemperaturen heraus, die sich gegenüber der ursprünglichen Umwandlungstemperaturen nur relativ gering verschieben. Dies ist u.a. bei der 40:60 Mischung zu sehen, wo sich ein Absinken der Schallgeschwindigkeitszahl bei ca. $11^{\circ} \mathrm{C}$ und $33^{\circ} \mathrm{C}$ ausmachen läßt.

Von Halstenberg et al. [48] [49] lag bereits ein ausreichend guter Satz an Schallgeschwindigkeitsprofilen von DMPC/Cholesterin Mischungen vor. Zur Ergänzung wurden diese Messungen wiederholt und vervollständigt. In Abb. 3.10 sind die Schallgeschwindigkeitskurven der DMPC/Cholesterin Mischungen dargestellt. Deutlich ist eine Verbreiterung der Umwandlung mit steigendem Cholesteringehalt zu sehen, bis bei der 66:33 Suspension eine unmerkliche Umwandlung den Kurven kaum mehr zu entnehmen ist.

\subsubsection{Bestimmung der Breite der Umwandlung}

Die Breite der Umwandlung, wie sie in der Schallgeschwindigkeit auftritt, ist abhängig von der Zusammensetzung der Membran und damit vom Mischungsverhältnis von DMPC zu DPPC bzw. DMPC zu Cholesterin. Um ein Maß für die Breite zu finden, wird zuerst an den Teil der Meßwerte unterhalb der Umwandlungstemperatur $T_{c}$ ein Polynom beliebigen Grades angepaßt (üblicherweise dritten oder vierten Grades). Mit den Meßwerten oberhalb vom $T_{c}$ wird analog verfahren. Die Auswahl und die Beurteilung, welche Punkte noch zu den Temperaturen unterhalb $T_{c}$ bzw. oberhalb $T_{c}$ gehören, liegt dabei im menschlichen Ermessen. Beide so bestimmten Polynome schneiden sich im Idealfall an der Umwandlungstemperatur, so daß dieser Schnittpunkt mittels des Newton-Verfahrens ermittelt werden kann. Ausgehend von diesem Punkt wurde durch einen vorher festgelegten, immer gleichen Abstand in Richtung zu tieferen und höheren Temperaturen die Breite der Umwandlung bestimmt. Die so ermittelten Breiten können selbstverständlich nur zu qualitativen Aussagen herangezogen werden. Jedoch ist ein Vergleich der Werte untereinander sehr gut möglich.

Auf diese Weise wurden die Breiten der Umwandlung der Suspensionen DMPC/DPPC, DMPC/DLPC und die der DMPC/Cholesterol Suspensionen bestimmt, wobei einerseits die Kurven aus Abb. 3.10 herangezogen wurden, andererseits ebenfalls die von S. Halstenberg ermittelten Werte ausgewertet wurden [49].

Abb. 3.11 zeigt die Breiten der DMPC/DPPC und DLPC/DMPC Mischungen. Es ist $\mathrm{zu}$ sehen, daß die Breite der Umwandlung jeweils bei den 50:50 Mischungen maximal wird und zu den 100:0 Mischungsverhältnissen abfällt. Weiter ist durch die erhöhte Kooperativität zu beobachten, daß die Breite der Umwandlung von reinem DPPC geringer ist, als die von reinem DMPC.

Wie zu erwarten, steigt in Abb. 3.12 mit steigendem Cholesteringehalt die Breite an. Der Vergleich mit Abb. 3.11 zeigt einen Ansteig weit über die Werte aus Abb. 3.11. Leider konnten von den Schallgeschwindigkeitszahlen der Mischungen mit Cholesteringehalt größer als $20 \%$ keine Breiten bestimmt werden, da ein charakteristisches Minimum, wie bei den anderen Messungen, nicht mehr auszumachen war. Zudem zeigt die Auftragung der Ergebnisse aus Abb. 3.10 und von S. Halstenberg eine gute Übereinstimmung der Breiten. 


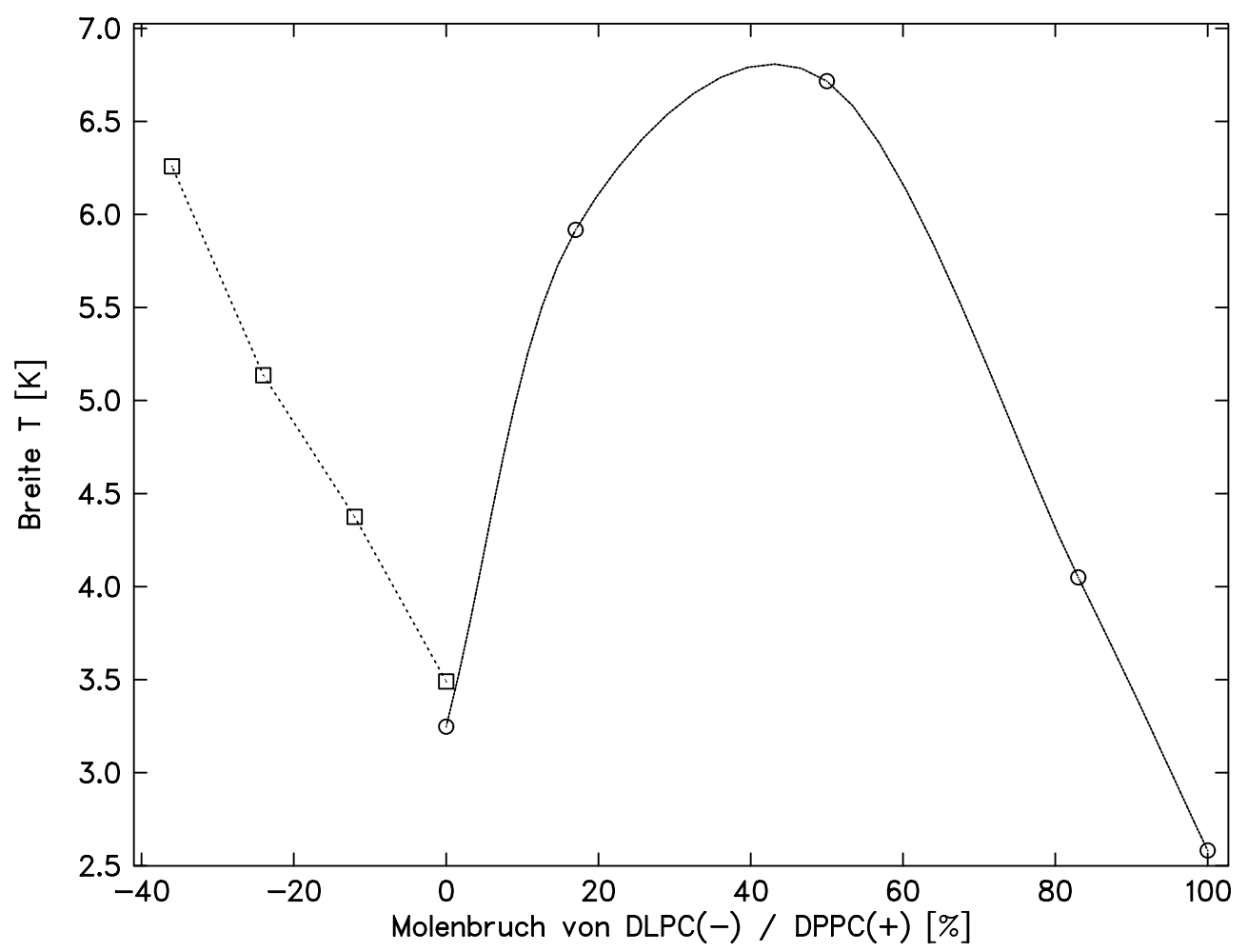

Abb. 3.11: Breite der Umwandlungen der DMPC/DPPC und DMPC/DLPC Mischungen, positive Werte bezeichnen den DPPC Anteil, während negative Werte den DLPC Anteil darstellen

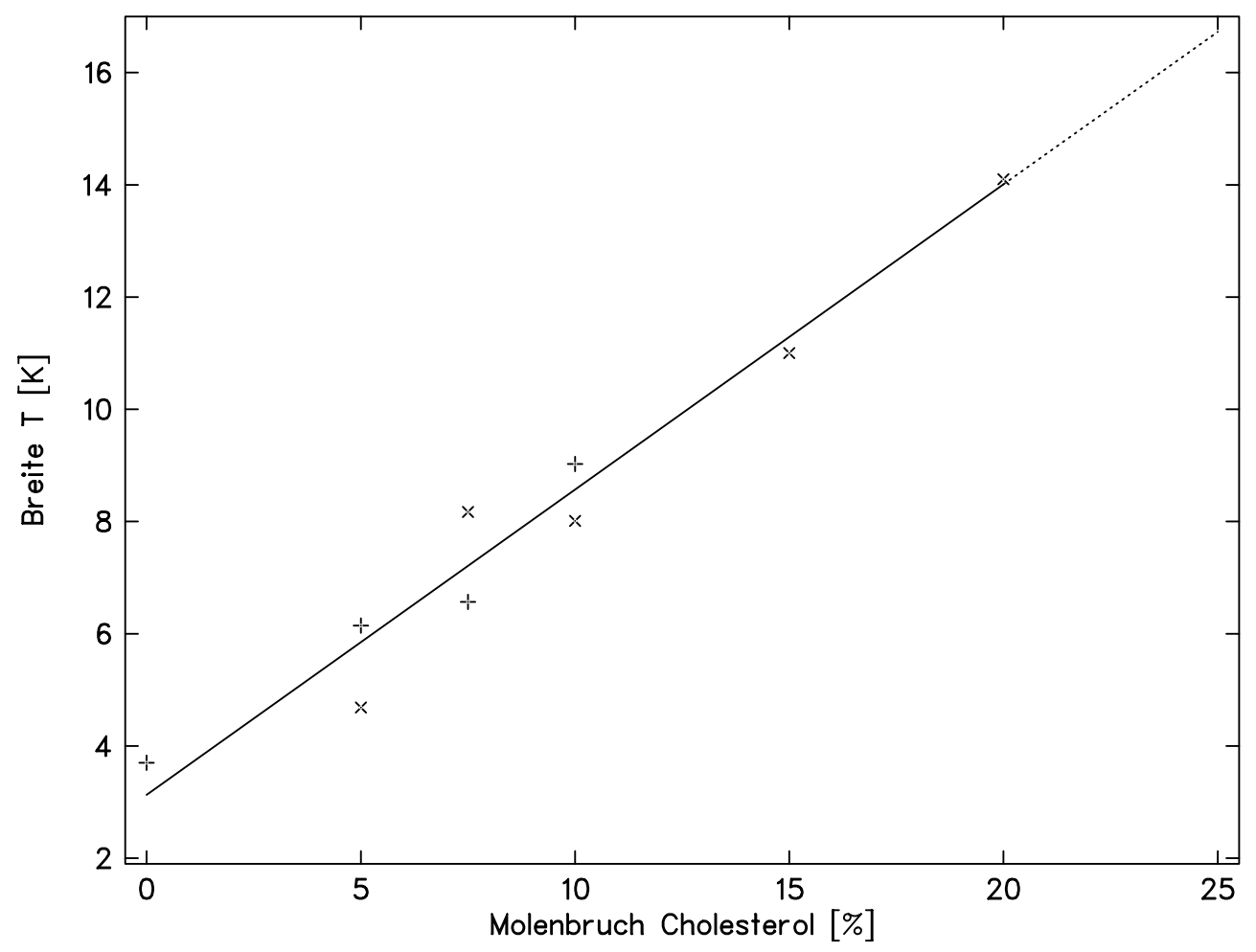

Abb. 3.12: Breite der Umwandlungen der DMPC/Cholesterol Mischungen, + Daten aus Abb. 3.6, × Daten von S. Halstenberg [49] 


\subsection{Die Theorie von T. Heimburg}

Die gemessenen Wärmekapazitätskurven und die Schallgeschwindigkeitskurven sind über die adiabatische Kompressibilität direkt miteinander verbunden. Mittels einiger theoretischer Überlegungen von T. Heimburg [53] [49] soll nun die Verbindung zwischen diesen Größen dargestellt werden und es sollen aus den gemessenen kalorimetrischen Kurven theoretische Kurven der Schallgeschwindigkeitszahl berechnet werden.

Beginnen wir mit der Wärmekapazität und der isothermen Volumenkompressibilität. Durch das Fluktuations-Dissipations-Theorem sind die Wärmekapazität $C_{p}$ und die isotherme Volumenkompressibilität $\kappa_{T}$ mit der Fluktuation von Enthalpie und Volumen eines Ensembles verknüpft:

$$
\begin{aligned}
C_{p} & =\left(\frac{d \bar{H}}{d T}\right)_{p}=\frac{\overline{H^{2}}-\bar{H}^{2}}{R T^{2}}, \\
\kappa_{T} & =-\frac{1}{\bar{V}}\left(\frac{d \bar{V}}{d p}\right)_{T}=\frac{\overline{V^{2}}-\bar{V}^{2}}{\bar{V} \cdot R T} .
\end{aligned}
$$

Stellt man $H$ als Summe der inneren Membranwärme $H_{0}(T)$ und einer durch Kettenumwandlungen erzeugten zusätzlichen Wärme $\Delta H(T)$ dar, so kann man die Wärmekapazität in einen Hintergrundanteil und einen durch die Alkylkettenumwandlungen erzeugten Anteil $\Delta C_{p}$ aufspalten:

$$
C_{p}=\left(\frac{d H_{0}}{d T}\right)_{p}+\left(\frac{d(\Delta H)}{d T}\right)_{p}=C_{p, 0}+\Delta C_{p} .
$$

Ebenso kann man die Kompressibilität in einen Grundanteil und einen mit den Fluktuationen verknüpften Anteil aufspalten:

$$
\kappa_{T}=-\left(\frac{1}{V} \frac{d V_{0}}{d p}\right)_{T}-\left(\frac{1}{V} \frac{\Delta V}{d p}\right)_{T}=\kappa_{T, 0}+\Delta \kappa_{T} .
$$

Mit den Maxwell-Relationen kann man die adiabatische Kompressibilität als Funktion von isothermer Kompressibilität, Wärmekapazität und der Volumenausdehnung darstellen:

$$
\kappa_{s}=\kappa_{T}-\frac{T}{\bar{V} C_{p}}\left(\frac{d \bar{V}}{d T}\right)_{p}^{2} .
$$

Im allgemeinen sind Kompressibilität, Wärmekapazität und Volumenausdehnung unabhängige Größen, die jeweils experimentell ermittelt werden müssen. Bei Lipidmembranen im Umwandlungsbereich ist es möglich, eine Abhängigkeit dieser Größen untereinander zu finden. Die Änderung der Kompressibilität hängt fast ausschließlich von Kettenumwandlungen ab. Anthony et al. [2] zeigten durch Dichtemessungen, daß im Umwandlungsbereich für Lipide und Lipidmischungen die Enthalpieänderung $\Delta H$ proportional zur Volumenänderung $\Delta V$ ist:

$$
\overline{\Delta V(T)}=\gamma_{v o l} \overline{\Delta H(T)}
$$




$$
\frac{d(\bar{\Delta} V)}{d T}=\gamma_{v o l} \frac{d(\bar{\Delta} H)}{d T}=\gamma_{v o l} \Delta C_{p}
$$

Unter der Annahme, daß dieses ebenso in einem größeren Temperaturbereich gegeben ist, zeigt Heimburg sogar die Gültigkeit für alle möglichen Zustände des Systems und schließt daraus, daß auch nicht trivialer Weise $\overline{\Delta V^{2}}=\gamma_{v o l}^{2} \overline{\Delta H^{2}}$ gilt. Daraus folgt mit den Gleichungen (3.10) und (3.13):

$$
\Delta \kappa_{T}=\frac{\gamma^{2} T}{\bar{V}} \Delta C_{p}
$$

Hier wird die empirische Proportionalitätskonstante von $\gamma=8,9 \cdot 10^{-4} \mathrm{~cm}^{3} / \mathrm{J}$ angenommen [53]. Je nach absolutem Wassergehalt der Probe nimmt die adiabatische Kompressibilität des Gesamtsystems eine Größe an zwischen einem Wert, der durch $\Delta C_{p}$ bestimmt wird, und $\infty$ an. Das heißt, um adiabatische Kompressibilität messen zu können, muß die Wärme $\Delta H$ von der Umgebung mit $C_{p}>\Delta C_{p}$ aufgenommen werden. Im Fall von vollständiger Relaxation und großem Wasseranteil gilt $\kappa_{T}=\kappa_{s}$. Nahe der Umwandlungstemperatur entsteht die Wärme hauptsächlich in der Membran, und nur bei sehr langsamer Messung findet ein kompletter Wärmeaustausch statt, die Messung ist also frequenzabhängig. Für sehr hohe Frequenzen ist dementsprechend $C_{p}=\Delta C_{p}$ (und damit $\kappa_{s}=0$ ). Bei hoher Frequenz, wie bei Ultraschallmessungen, kann man die Membran und ihr Lösungsmittel als thermisch entkoppelt getrennt ansehen. Deswegen sind die adiabatisch Kompressibilitäten von Wasser und Lipid additiv, bei Mischungen mit Volumenanteilen $f_{\mathrm{H}_{2} \mathrm{O}}$ und $f_{\text {lipid }}$ gilt:

$$
\kappa_{s}=f_{\mathrm{H}_{2} \mathrm{O}} \cdot \kappa_{s, H_{2} \mathrm{O}}+f_{\text {lipid }} \cdot \kappa_{\text {s,lipid }} .
$$

Um die adiabatische Kompressibilität zu berechnen, kann man Gleichung (3.17) in (3.14) einsetzen. Unter Vernachlässigung des Teils der Ausdehnung des Lipidvolumens, der nicht durch Kettenisomerisierungen verursacht wird, gilt wie folgt:

$$
\begin{aligned}
\kappa_{s}^{l i p i d} & =\kappa_{T, 0}^{l i p i d}+\Delta \kappa_{T}-\frac{T}{\bar{V}^{l i p i d} C_{p}^{l i p i d}}\left(\frac{d \bar{V}^{l i p i d}}{d T}\right)_{p}^{2} \\
\kappa_{s}^{l i p i d} & =(1-f) \cdot \kappa_{T, \text { gel }}+f \cdot \kappa_{T, \text { fluid }}+\Delta \kappa_{T}-\frac{T}{\bar{V}^{l i p i d} C_{p}^{l i p i d}}\left(\frac{d \bar{V}^{l i p i d}}{d T}\right)_{p}^{2} \\
& \approx(1-f) \cdot \kappa_{T, \text { gel }}+f \cdot \kappa_{T, \text { fluid }}+\Delta \kappa_{T}\left(1-\frac{\Delta C_{p}}{C_{p}^{l i p i d}}\right) .
\end{aligned}
$$

Hier ist $f$ der Anteil des Lipides, der sich schon im fluiden Zustand befindet und $C_{p}^{\text {lipid }}$ die Wärmekapazität der hydrophoben Ketten, die als unabhängig vom sonstigen Schmelzprozeß betrachtet werden kann. Berücksichtigt man den Anteil der Volumenausdehnung, der nicht durch die Kettenisomerisierungen verursacht wird, so gilt

$$
\frac{d V}{d T}=\gamma \cdot \Delta C_{p}+\left.\frac{d V}{d T}\right|_{0}
$$


Es zeigt sich, daß die Verwendung von Gleichung (3.22) in Gleichung (3.19) nur einen unwesentlichen zusätzlichen Beitrag zu den Schallgeschwindigkeitskurven liefert, diese jedoch im Bereich der Umwandlung ein wenig "runder" erscheinen läßt und somit zu einer besseren Übereinstimmung mit den gemessenen Kurven führt. Bei den Berechnungen wird $C_{p}^{l i p i d}=1650 \mathrm{~J} /(\mathrm{mol} \mathrm{K})$ angenommen [13]. $\kappa_{T, \text { gel }}$ und $\kappa_{T, \text { fluid }}$ sind die intrinsischen Kompressibilitäten.

Letztendlich soll es das Ziel sein, aus der Wärmekapazität die Schallgeschwindigkeit auszurechnen. Dazu muß zunächst die Enthalpie aus der gemessenen $C_{p}$-Kurve berechnet werden:

$$
\Delta H_{0}=\int_{T_{1}}^{T_{2}} \Delta C_{p}(T) d t \quad .
$$

Dafür wird an kleine Teilbereiche der $C_{p}$-Kurven stückweise ein Polynom zweifachen Grades angepaßt und dieses dann analytisch integriert. Mittels der schon berechneten Enthalpie kann sehr einfach die sogenannte "fluid fraction" $f$, der Anteil, der schon im fluiden Zustand ist, berechnet werden:

$$
f=\frac{\Delta H(T)}{\Delta H_{0}}=\frac{\int_{T_{1}}^{T} \Delta C_{p}(T) d t}{\int_{T_{1}}^{T_{2}} \Delta C_{p}(T) d t} .
$$

Die Schallgeschwindigkeit selbst ergibt sich zu:

$$
c=\sqrt{\frac{1}{\rho \kappa_{s}}} .
$$

Mit Hilfe von Gleichung (3.18) und (3.21) läßt sich nun die Schallgeschwindigkeit berechnen. Die Kompressibilitäten $\kappa_{T, \text { gel }}$ und $\kappa_{T \text {,fluid }}$ sind dabei Parameter die von System zu System verschieden sind. Um die Temperaturabhängigkeit der Kompressibilitäten hinreichend zu berücksichtigen, werden keine konstanten Werte für $\kappa_{T, g e l}$ und $\kappa_{T, \text { fluid }}$ angenommen, sondern eine lineare Abhängigkeit von der Temperatur verwendet.

$$
\begin{gathered}
\kappa_{T, \text { gel }}=\kappa_{B, \text { gel }}+T \cdot \kappa_{m, \text { gel }} \\
\kappa_{T, \text { fluid }}=\kappa_{B, \text { fluid }}+T \cdot \kappa_{m, \text { fluid }} .
\end{gathered}
$$

Es zeigt sich, daß sich die jeweiligen Kompressibilitäten der Gel-Phase bzw. der fluiden Phase zusammenfassend beschreiben lassen, also innerhalb einer Meßserie von der Zusammensetzung der Membran unabhängig sind. Sie werden mittels Anpaßrechungen an die gemessenen Kurven eines Datensatzes bestimmt und sind in Tabelle 3.2 aufgeführt. Danach ergeben sich zwar für die unterschiedlichen Systeme DMPC/DPPC, DMPC/DMG und DLPC/DPPC verschiedene lineare Abhängigkeiten, jedoch sind diese derart gelegen, daß z.B. für die Suspension DMPC/Wasser die Kompressibilitäten aus den entsprechenden Systemen in etwa gleich sind. So ergeben sich über einen weiten Temperaturbereich sinnvolle Werte für die Kompressibilität. Einzig bei den DLPC/DPPC Systemen, bei denen die Abhängigkeiten "nur" aus Schallgeschwindigkeitsmessungen oberhalb von $10^{\circ} \mathrm{C}$ bestimmt werden können, ergeben sich für Temperaturen unterhalb von $5^{\circ} \mathrm{C}$ negative Kompressibilitäten. Der Vollständigkeit halber werden aber die Wärmekapazitätskurven der DLPC/DPPC 


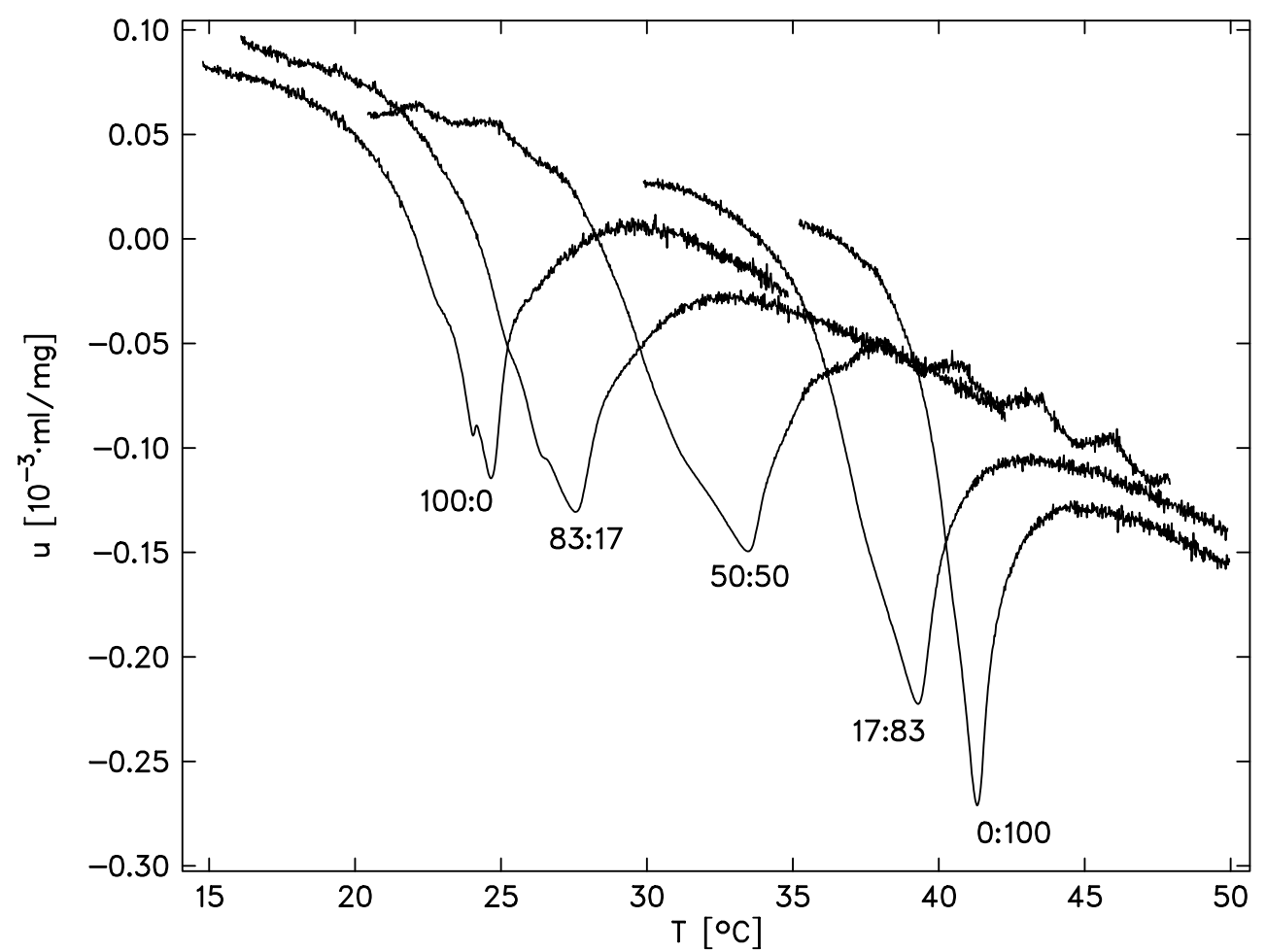

Abb. 3.13: Temperaturprofile der aus den $C_{p}$-Daten berechneten Schallgeschwindigkeitszahlen der DMPC/DPPC Suspensionen

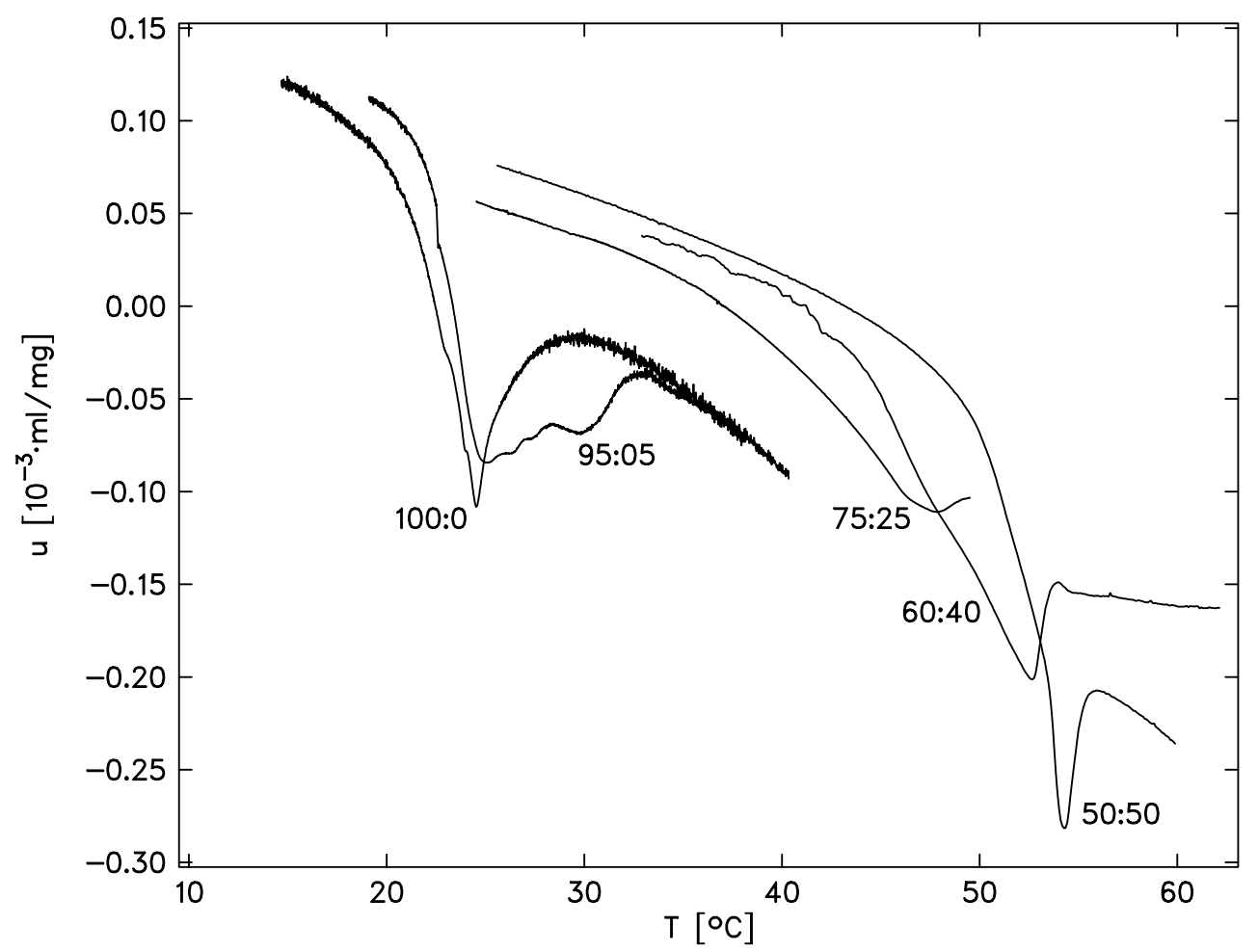

Abb. 3.14: Temperaturprofile der aus den $C_{p}$-Daten berechneten Schallgeschwindigkeitszahlen der DMPC/DMG Suspensionen 


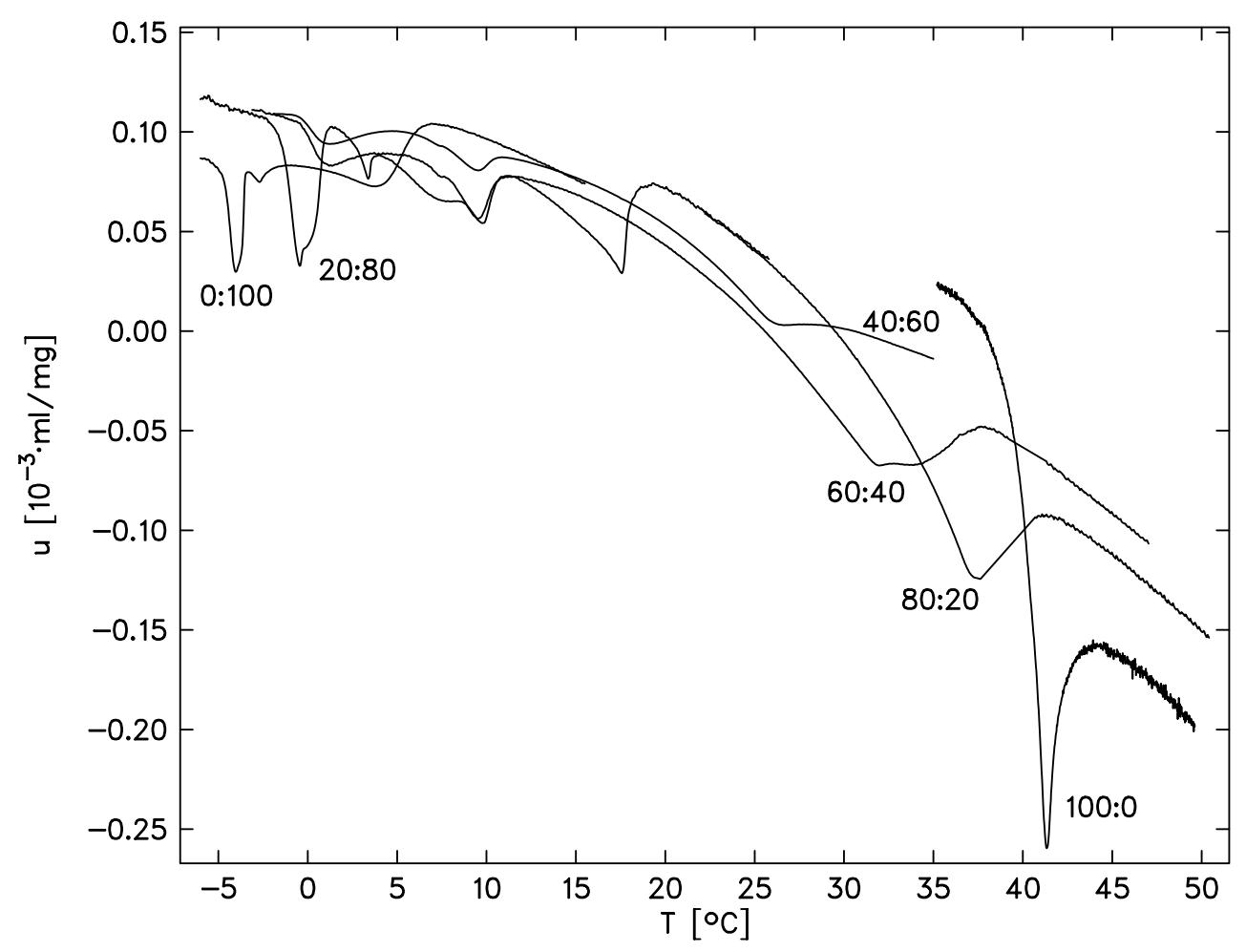

Abb. 3.15: Temperaturprofile der aus den $C_{p}$-Daten berechneten Schallgeschwindigkeitszahlen der DLPC/DPPC Suspensionen

\begin{tabular}{|c|c|c|c|c|}
\hline & $\begin{array}{c}\kappa_{B, \text { gel }} \\
{\left[\mathrm{cm}^{3} / \mathrm{J}\right]}\end{array}$ & $\begin{array}{c}\kappa_{\text {m,gel }} \\
{\left[\mathrm{cm}^{3} / \mathrm{JK}\right]}\end{array}$ & $\begin{array}{c}\kappa_{B, \text { fluid }} \\
{\left[\mathrm{cm}^{3} / \mathrm{J}\right]}\end{array}$ & $\begin{array}{c}\kappa_{\text {m,fluid }} \\
{\left[\mathrm{cm}^{3} / \mathrm{JK}\right]}\end{array}$ \\
\hline DMPC/DPPC & $-7,7 \cdot 10^{-5}$ & $8,45 \cdot 10^{-6}$ & $-2,3 \cdot 10^{-4}$ & $2,1 \cdot 10^{-5}$ \\
DMPC/DMG & $-2,6 \cdot 10^{-4}$ & $1,3 \cdot 10^{-5}$ & $-3,1 \cdot 10^{-4}$ & $2,5 \cdot 10^{-5}$ \\
DLPC/DPPC & $-1,4 \cdot 10^{-4}$ & $8,5 \cdot 10^{-6}$ & $-5,6 \cdot 10^{-4}$ & $3,2 \cdot 10^{-5}$ \\
\hline
\end{tabular}

Tabelle 3.2: Kompressibilitäten der Membranen im gelförmigen und fluiden Zustand. Die Temperaturverläufe werden durch lineare Abhängigkeiten gemäß Gleichung (3.27) und (3.26)beschrieben. 
100:0 und 80:20 auch mit diesen Werten in Schallgeschwindigkeitskurven umgerechnet. Höchstwahrscheinlich sind die negativen Kompressibilitäten als nahe bei Null liegende Werte zu interpretieren oder die lineare Abhängigkeit ist bei diesem System bei Temperaturen nahe Null nicht mehr gegeben.

In Abb. 3.13 sind die so berechneten Schallgeschwindigkeitszahlen für die DMPC/DPPC Suspensionen dargestellt. Der Vergleich mit der gemessenen Daten aus Abb. 3.6 zeigt eine sehr gute Übereinstimmung.

In Abb. 3.14 sind die berechneten Schallgeschwindigkeitszahlen der DMPC/DMG Mischungen dargestellt. Der Vergleich mit den gemessenen Daten aus Abb. 3.8 zeigt auch hier eine sehr gute Übereinstimmung, wenn auch die Daten der 75:25 Mischung eine kleine Verschiebung gegenüber der Meßwerten aufweisen. Auch fällt auf, daß bei den Mischungen 60:40 und 50:50 der Schallgeschwindigkeitseinbruch in der Nähe der Umwandlungstemperatur bei den berechneten Kurven schmaler ausfällt als bei den direkt gemessenen Kurven. Suspensionen aus DMPC und DMG mit hohem DMG Anteil neigen zu starkem Ausfallen und Absinken der Lipidaggregate. Zudem zeigt das Phasendiagramm in Abb. 2.6, daß sich bei derart hohem DMG Anteil die Lipide zunehmend nicht mehr in der $L_{\alpha}$-Phase (Membran-Phase), sondern in der $H_{\alpha}$-Phase, die auch als hexagonale Phase bezeichnet wird, befinden. Dieses führt wahrscheinlich dazu, daß diverse zusätzliche bzw. andere thermodynamische Prozesse ablaufen, als die in den obigen Überlegungen dargestellten, da die laterale Anordnung der Lipiddoppelschicht in dieser Form nicht mehr vorliegt.

Abb. 3.15 zeigt die aus den $C_{p}$-Kurven berechneten Schallgeschwindigkeitszahlen der DLPC/DPPC Suspensionen. Auch hier zeigt der Vergleich mit den real gemessenen Werten eine gute Übereinstimmung (Abb. 3.9), obwohl die kalorimetrischen Messungen mit multilammellaren Vesikeln und die Schallgeschwindigkeitsmessungen mit unilammellaren Vesikeln getätigt wurden.

\subsection{Kompressibilität und Fluktuationen}

Sofern die Dichte in Gleichung (3.25) eine bekannte Größe sein sollte, ist es prinzipiell möglich die adiabatische Kompressibilität aus einer Schallgeschwindigkeitsmessung zu errechnen. Definiert man wie oben in Gleichung (3.9) analog zur Schallgeschwindigkeitszahl für die Dichte $\rho$ bzw. für die adiabatische Kompressibilität $\kappa_{s}$ eine "Dichtezahl" bzw. "Kompressibilitätszahl"

$$
r=\frac{\rho_{\text {Lipid }}-\rho_{\text {Wasser }}}{\rho_{\text {Wasser }} \cdot k}, \quad s=\frac{\kappa_{\text {Lipid }}-\kappa_{\text {Wasser }}}{\kappa_{\text {Lipid }} \cdot k},
$$

so kann man durch Differentation der Gleichung (3.25) nach der Konzentration folgende Beziehung zwischen den Inkrementen erhalten [129]:

$$
s=-2 \cdot u-r
$$

Eine weitere wichtige Größe bei der Betrachtung von Phasenumwandlungen von Membranen ist das "scheinbare molare Partialvolumen" der Komponente A:

$$
V_{A}^{m}=\left(\frac{\partial V}{\partial n_{A}}\right)_{T, P, n_{B}}
$$




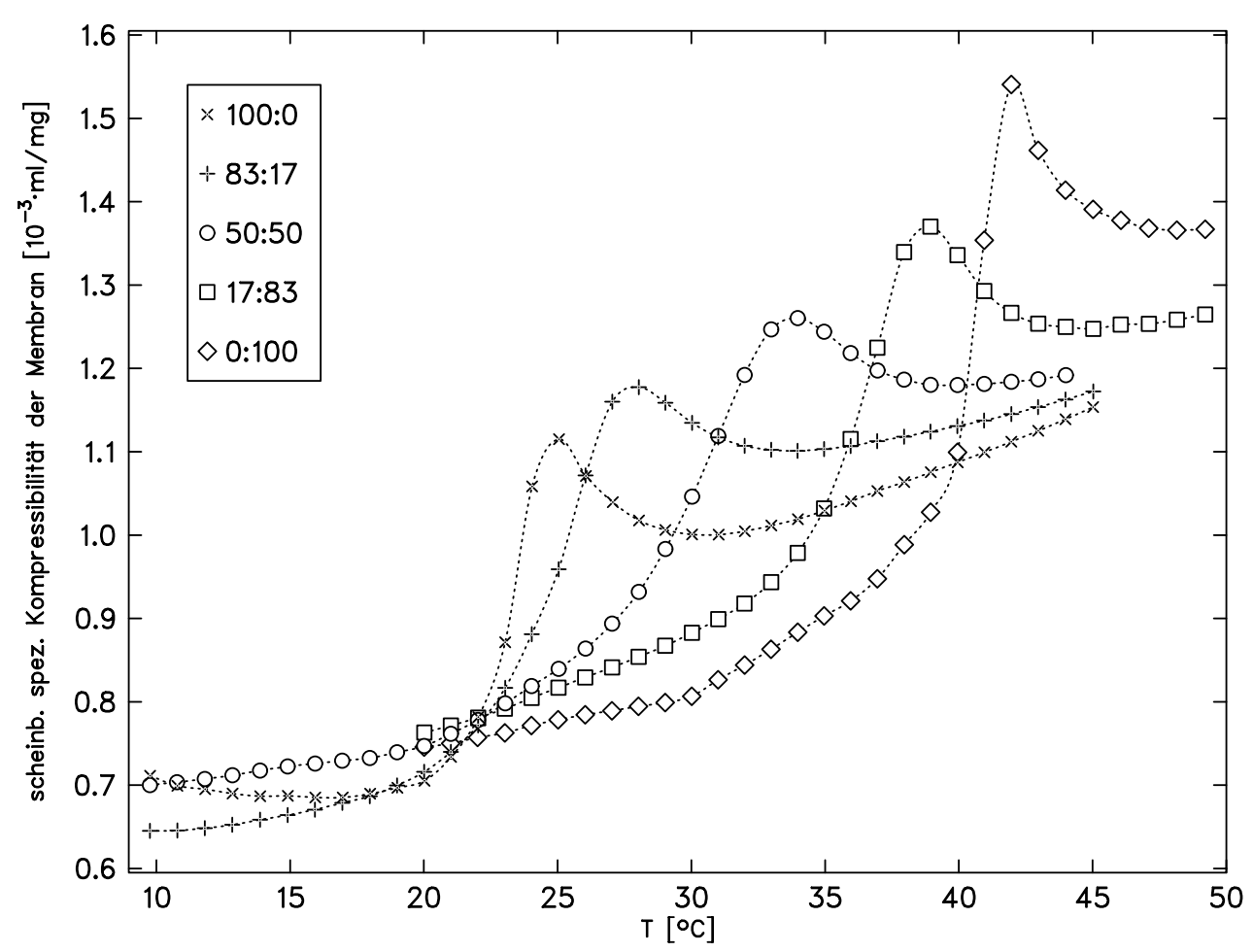

Abb. 3.16: Scheinbare spezifische adiabatische Kompressibilität der DMPC/DPPC Suspensionen

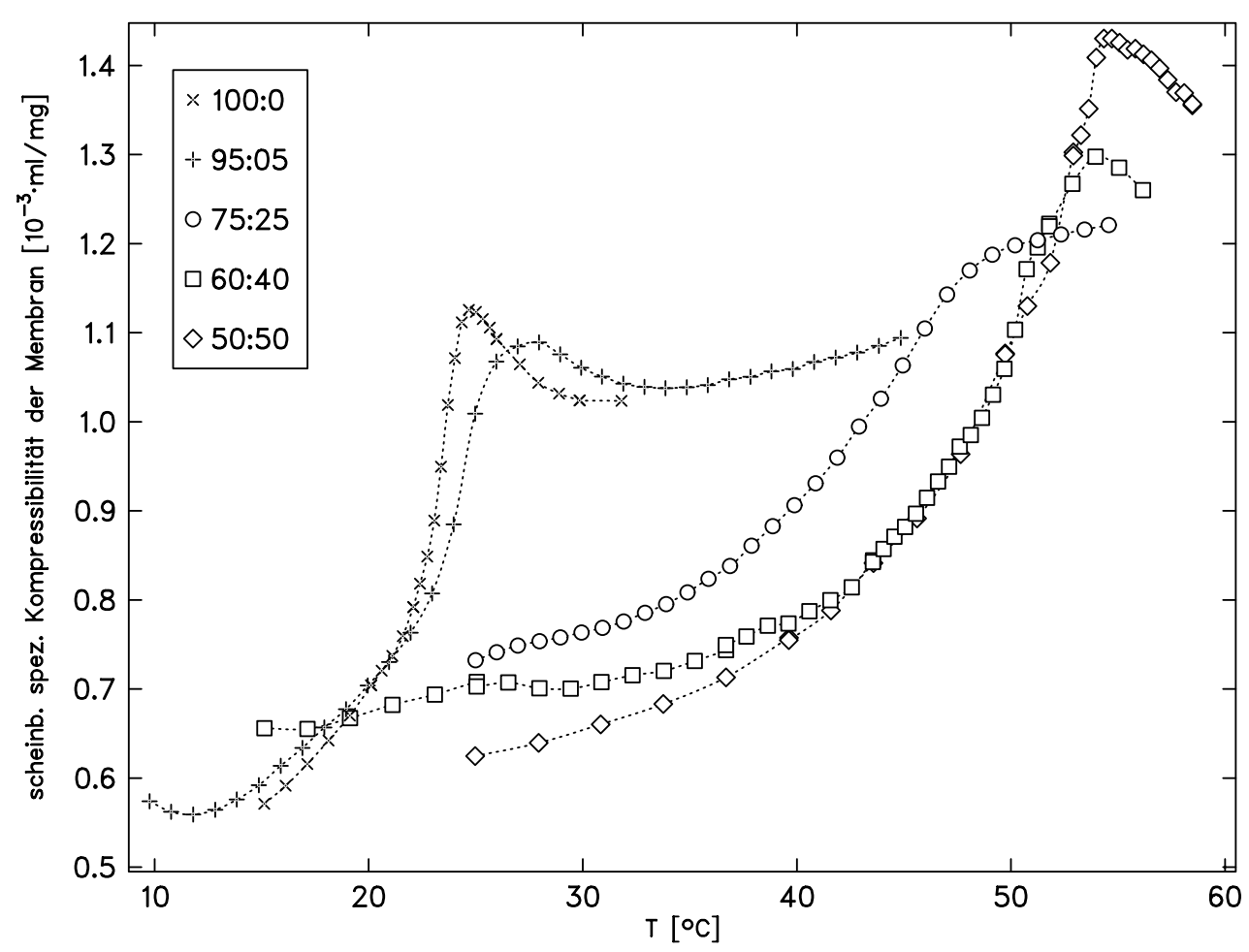

Abb. 3.17: Scheinbare spezifische adiabatische Kompressibilität der DMPC/DMG Suspensionen 


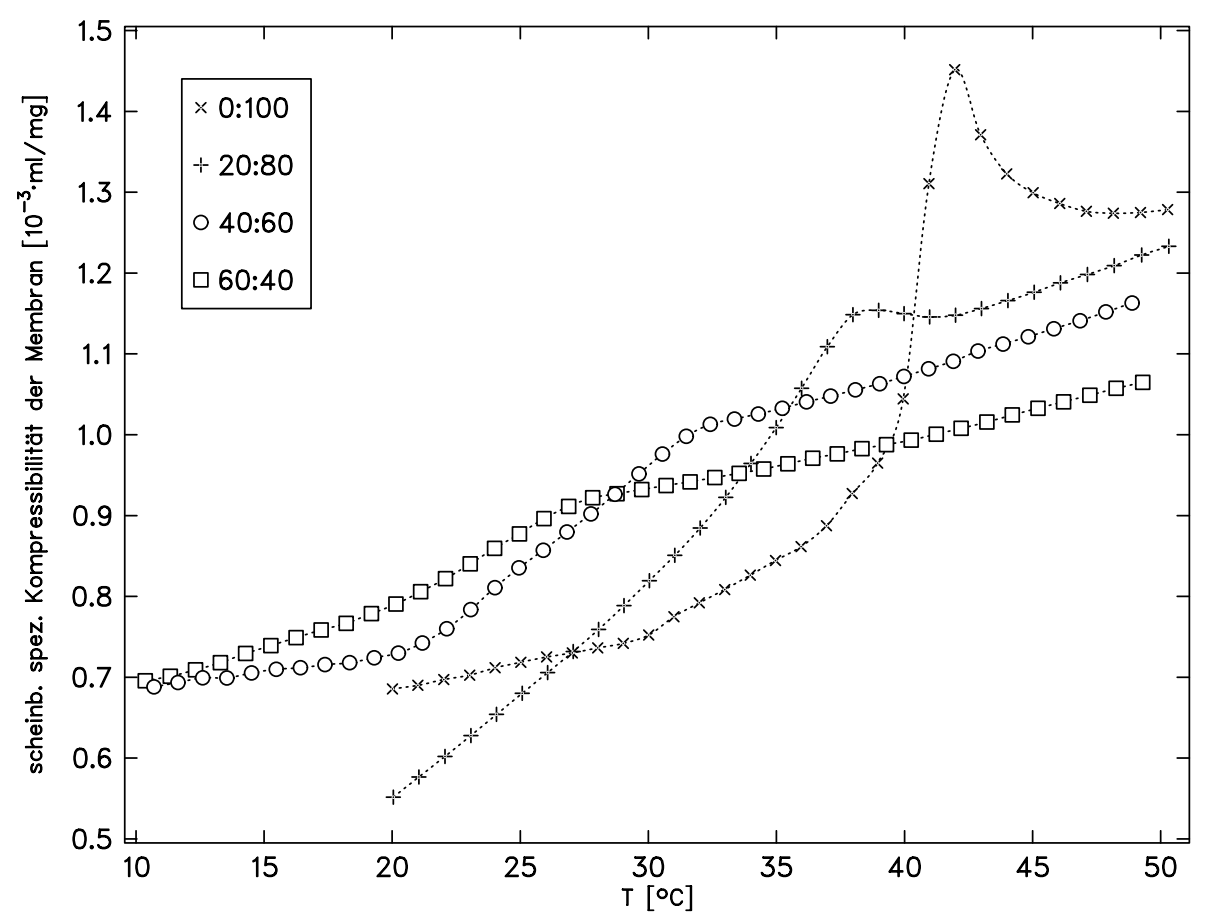

Abb. 3.18: Scheinbare spezifische adiabatische Kompressibilität der DLPC/DPPC Suspensionen

Es beschreibt die Änderung des Volumens einer Lösung bei Zugabe eines Moles der Komponente A. Hieraus läßt sich das auf die Gewichtskonzentration k bezogene "scheinbare spezifische Partialvolumen" $\phi_{V}$ herleiten:

$$
\phi_{V}=\frac{1}{\rho_{\text {Wasser }}}\left(1-\frac{\rho_{\text {Lipid }}-\rho_{\text {Wasser }}}{k}\right)=\frac{1}{\rho_{\text {Wasser }}}-r .
$$

Analog läßt sich mit Hilfe der "adiabatischen Kompressibilität des Volumens V"

$$
K_{\text {Lipid }}=-\left(\frac{\partial V}{\partial P}\right)_{\text {Lipid }}=\kappa_{\text {Lipid }} \cdot V
$$

die scheinbare molare adiabatische Kompressibilität $\Phi K_{\text {Lipid }}$

$$
\Phi K_{\text {Lipid }}=\frac{1}{V}\left(\frac{\partial K_{\text {Lipid }}}{\partial C}\right)_{C \Rightarrow 0}=\frac{K_{\text {Lipid }}-K_{\text {Wasser }}}{C V}
$$

mit der molaren Konzentration C nach A. P. Sarvazyan [129] für kleine Konzentrationen durch

$$
\frac{\Phi K_{\text {Lipid }}}{\kappa_{\text {Wasser }}}=\frac{1}{\kappa_{\text {Wasser }}} \cdot \frac{\kappa_{\text {Lipid }} V_{\text {Lipid }}-\kappa_{\text {Wasser }} V_{\text {Wasser }}}{k V_{\text {Lipid }}}
$$

darstellen. Mit der Gewichtskonzentration k läßt sich dementsprechend die scheinbare spezifische adiabatische Kompressibilität $\phi_{k}$ ausdrücken:

$$
\phi_{k}=\frac{1}{\rho_{\text {Wasser }}}-2 u-2 r
$$

Für die Suspensionen DMPC/DPPC und DMPC/DMG aller Mischungen liegen Dichtemessungen von F. Wente und M. Höckel mit ausreichender Genauigkeit vor 
[148] [65]. Mit Hilfe des daraus folgenden scheinbaren spezifischen Volumens ist es möglich, mittels Gleichung (3.35) die scheinbare spezifische adiabatische Kompressibilität zu errechnen (Abb. 3.16 und 3.17). In Tabelle 3.3 sind die scheinbaren spezifischen Volumina sowohl unterhalb als auch oberhalb der entsprechenden Umwandlungstemperatur der verschiedenen Suspensionen aufgeführt. Für die DLPC/DPPC Mischungen liegen keine entsprechenden Messungen vor und es wird deshalb von $\phi_{V, T<T_{m}}=0,93 \frac{\mathrm{cm}^{3}}{\mathrm{~g}}$ und $\phi_{V, T>T_{m}}=0,98 \frac{\mathrm{cm}^{3}}{\mathrm{~g}}$ ausgegangen. Der Temperaturverlauf wird mittels der "fluid-fraction" anhand eine Wärmekapazitätskurve errechnet.

\begin{tabular}{|c|c|c|c||c|c|c|c|}
\hline DMPC & DPPC & $\begin{array}{c}\phi_{V, T<T_{m}} \\
{\left[\mathrm{~cm}^{3} / \mathrm{g}\right]}\end{array}$ & $\begin{array}{c}\phi_{V, T>T_{m}} \\
{\left[\mathrm{~cm}^{3} / \mathrm{g}\right]}\end{array}$ & DMPC & DMG & $\begin{array}{c}\phi_{V, T<T_{m}} \\
{\left[\mathrm{~cm}^{3} / \mathrm{g}\right]}\end{array}$ & $\begin{array}{c}\phi_{V, T>T_{m}} \\
{\left[\mathrm{~cm}^{3} / \mathrm{g}\right]}\end{array}$ \\
\hline 100 & 0 & 0,930 & 0,986 & 100 & 0 & 0,930 & 0,986 \\
83 & 17 & 0,933 & 0,995 & 95 & 5 & 0,920 & 0,980 \\
50 & 50 & 0,948 & 1,002 & 75 & 25 & 0,890 & 0,980 \\
17 & 83 & 0,958 & 1,008 & 60 & 40 & 0,880 & 0,970 \\
0 & 100 & 0,960 & 1,016 & 50 & 50 & 0,870 & 0,960 \\
\hline
\end{tabular}

Tabelle 3.3: Scheinbare spezifische Volumina unterhalb und oberhalb der entsprechenden Umwandlungstemperaturen [148] [65]

\subsection{Strukturelles Verhalten}

Gleichung (3.10) beschreibt den Schmelzvorgang der Membranen durch kritische Fluktuationen der Enthalpie und des Volumens. Wie die Meßergebnisse zeigen, sind genau in der Umwandlung die Wärmekapazität und die Kompressibilität maximal und damit auch die Fluktuationen. Diese Fluktuationen der Enthalpie und des Volumens lassen sich letztendlich auf Fluktuationen der "fluid fraction", also dem Verhältnis zwischen schon geschmolzenen und noch nicht geschmolzenem Lipid zurückführen. Aus Monte-Carlo-Simulationen des Schmelzvorganges einer Membran ist bekannt, daß sich sogenannte Domänen auf der Membran bilden, die fast ausschließlich aus entweder fluiden oder gelförmigen Lipid bestehen [54] [44] [68] [137]. Diese Domänen zerfallen und bilden sich ständig neu. Das Verhältnis der fluiden Domänen zu den gelförmigen Domänen schwankt dabei fortwährend und ist somit Ausdruck der Fluktuationen.

Wie aus den Wärmekapazitäts- und Schallgeschwindigkeitsmessungen ersichtlich, sinkt die Amplitude der Fluktuationen zunächst ausgehend von reinem DMPC bei Zugabe von DLPC bzw. DPPC. Reines DMPC schmilzt bei etwa $24{ }^{\circ} \mathrm{C}$ und DPPC bei etwa $42{ }^{\circ} \mathrm{C}$ verursacht durch die unterschiedliche Kohlenstoffkettenlänge. Liegt ein Mischungsverhältnis beider Lipide in der Membran vor, so bilden sich bei geeigneter Zwischentemperatur Domänen, die sowohl fluides DMPC, als auch fluides DPPC bzw. DLPC enthalten. Bei reinem DPPC ist die Amplitude der Fluktuationen größer als bei reinem DMPC, was der erhöhten Kooperativität von DPPC zugeschrieben wird.

Bei Zugabe von Cholesterin zu einer Membran, die ursprünglich aus nur einer Lipidsorte besteht, verringert sich die Amplitude der Fluktuationen mit steigendem 
Cholesteringehalt. Es wird vermutet, daß sich die Cholesterinmoleküle an den Grenzen zwischen den fluiden Domänen und den gelförmigen Domänen anlagern [43]. Somit werden die Übergänge zwischen den beiden Zuständen der Lipide zunehmend unterbunden, was zur Minderung der Fluktuationen führt.

Das ungewöhnliche Verhalten der DLPC/DPPC Suspensionen im Gegensatz zu den DMPC/DPPC und DLPC/DMPC Suspensionen deutet auf eine Entmischung der beiden Lipidsorten innerhalb einer Membran hin. Während die Kettenlängen bei den DMPC/DPPC und DLPC/DMPC Mischungen sich nur wenig unterscheiden, ist diese Differenz bei der DLPC/DPPC Mischung doppelt so hoch. Es scheinen fortwährend Phasen reinen DLPCs und DPPCs zu existieren, die in gewisser Unabhängigkeit bei ihren individuellen Umwandlungstemperaturen den Phasenübergang zeigen. Über einen sehr großen Temperaturbereich koexistieren somit gelförmige und fluide Lipide in einer Membran nebeneinander. So ist es zu erklären, daß die DLPC/DPPC 40:60 und DLPC/DPPC 60:40 Suspensionen jeweils zwei Umwandlungspunkte zeigen. Diese Überlegungen stützen sich u.a. auf Untersuchungen an DMPC/DSPC Membranen, bei denen ein ganz ähnliches Phasenverhalten vorliegt. DSPC besitzt eine Länge der Alkylketten von 18 Kohlenstoffatomen und hat somit eine entsprechend höhere Umwandlungstemperatur als DMPC bzw. DPPC, die bei etwa $56{ }^{\circ} \mathrm{C}$ liegt. Somit bildet DMPC/DSPC ein analoges System zu den hier gemessenen DLPC/DPPC Syspensionen, da sich bei beiden Mischungen die beiden Komponenten um $2 \mathrm{CH}_{2}$-Gruppen in der Länge der Alkylketten unterscheiden. Kalorimetrische Messungen und Monte-Carlo-Simulationen bestätigen das Bild der koexistierenden, entmischten Phasen bei DMPC/DSPC Suspensionen [68].

Die Beimischung von DMG zu einer DMPC-Membran verursacht im Gegensatz zu den DMPC/DLPC bzw. DMPC/DPPC Suspensionen ein gänzlich anderes Verhalten. Es zeigt sich mit steigendem DMG-Gehalt kein allmählicher Temperaturanstieg der Hauptumwandlung wie er z.B. bei den DMPC/DPPC Suspensionen zu beobachten ist. Da jedoch die Kohlenwasserstoffkettenlänge von dem verwendeten DMG exakt der von DMPC entspricht, müssen die Lipide in einer neuen Konfiguration vorliegen. Möglicherweise bindet das DMG relativ fest über eine Wasserstoffbrückenbindung an ein benachbartes DMPC-Molekül und bildet so eine neue Einheit, die einen relativ hohen Schmelzpunkt besitzt. So könnten also schon unterhalb der Umwandlungstemperatur Domänen vorliegen, die einerseits DMG und DMPC mit einem relativ festen Mischungsverhältnis beinhalten, und andererseits größere Flächen mit reinem DMPC. Diese beiden Bereiche durchmischen sich nur sehr schlecht und würden folglich getrennt voneinander schmelzen. Dies erklärt u.a. den Verlauf der Kurven der DMPC/DMG 75:25 Suspensionen in denen deutlich zwei getrennte Umwandlungstemperaturen auszumachen sind.

Über die Kalorimetrie und die Schallgeschwindigkeitsmessung ist man in der Lage, Aussagen über die Art und Weise der Fluktuationen und ihre Amplitude zu tätigen. Diese Meßmethoden sind somit eine sehr hilfreiche Methode zur Untersuchung des thermodynamischen Verhaltens von Membranen, insbesondere deren Phasenverhaltens. Jedoch sind Aussagen über die Geschwindigkeit dieser Prozesse bzw. das zeitliche Verhalten molekularer Vorgänge, insbesondere die Separation unterschiedlicher Relaxationsmechanismen nicht möglich. Dies gelingt erst durch den Einsatz der Ultraschallspektroskopie (siehe Kapitel 5). 


\section{Dielektrische Spektroskopie}

Viele Lipide enthalten als einen wesentlichen Bestandteil des Moleküls eine stark polare Kopfgruppe, die ein permanentes Dipolmoment [17] besitzt. Somit sind LipidLösungen sensitiv für die dielektrische Spektroskopie unter der Voraussetzung der Beweglichkeit dieser polaren Kopfgruppe. Das Dipolmoment der Kopfgruppen gestattet ein Studium der Anordnung und Dynamik der Kopfgruppen ohne Zugabe von sogenannten Markern, die die Membran mehr oder weniger beeinflussen. Die polaren Kopfgruppen treten in Wechselwirkung mit dem angelegten elektrischen Wechselfeld und erzeugen so eine makroskopische Polarisation der Lösung, die ihrerseits von der speziellen Anordnung der Lipide und vom thermischen Geschehen beeinflußt wird. Die Relaxation der Kopfgruppenumorientierung ist bereits in [46] [57] [120] [71] [79] [60] eingehend untersucht worden, allerdings mittels zeitaufwendiger Zeitbereichverfahren oder Admittanzbrückenmessungen. Die Möglichkeit in dem hier verwendeten Verfahren, relativ viele dielektrische Spektren in kurzer Zeit mit sehr hoher Genauigkeit aufzunehmen, kann jedoch neue Aspekte eröffnen, vor allem Dingen im Hinblick auf die Temperaturabhängigkeit der auftretenden Relaxationen. Zudem wurden unterschiedliche Zusammensetzungen von Membranen untersucht. Die hier durchgeführte dielektrische Spektroskopie dient auch zur weiteren Aufklärung und identifikation eines molekularen Prozesses, der im weiteren in der akustischen Spektroskopie auftritt (siehe Kapitel 7.3).

\subsection{Flüssigkeiten in elektrischen Wechselfeldern}

In der dielektrischen Spektroskopie von Flüssigkeiten versucht man, mit Hilfe der makroskopisch erfaßbaren Polarisation auf mikroskopische Bewegungen und Strukturbildungen der Moleküle zu schließen [75]. Wird an ein Medium ein elektrisches Wechselfeld

$$
\vec{E}(t)=\vec{E}_{0} \cdot e^{i \omega t}, \quad \omega=2 \pi f
$$

mit der Kreisfrequenz $\omega$ angelegt, so induziert dies im Medium u. a. eine Ausrichtung der vorhandenen permanenten Dipole. Das äußert sich makroskopisch in der Polarisation des Mediums

$$
\vec{P}(t)=\vec{P}_{0} \cdot e^{i \omega t+\varphi},
$$

die wegen zwischenmolekularer Wechselwirkungen zu dem angelegten elektrischen Feld phasenverschoben sein kann. Die Phase ist allgemein frequenzabhängig: $\varphi=$ $\varphi(\omega)$.

Zwei Mechanismen tragen zu Polarisation des Mediums bei. Zum einen werden Moleküle, die ein permanentes Dipolmoment besitzen, im elektrischen Feld umorientiert. Zum anderen werden Elektronen und Atomkerne in einem Molekül verschoben, so daß ein induzierter Dipol entsteht. Dieser zuletzt genannte Mechanismus wird Verschiebungspolarisation genannt. Er führt zu einer Absorption bzw. Dispersion oberhalb von $100 \mathrm{GHz}$ und spielt deshalb für die dielektrische Spektroskopie nur eine untergeordnete Rolle, da die verwendeten Meßapparaturen nicht in der Lage sind, in diesem Bereich zu messen. 
Die makroskopische meßbare Polarisation hat ihre Ursache in der mikroskopischen Ausrichtung der molekularen Dipolmomente $\vec{\mu}_{i}$ und ist gegeben durch

$$
\vec{P}(t)=\frac{1}{V} \cdot \sum_{i=1}^{N} \vec{\mu}_{i}(t)+\vec{P}_{\text {Verschieb }}
$$

N bezeichnet die Anzahl der dipolaren Gruppen $\vec{\mu}_{i}$ im Volumen V. Im statischen Fall, für hinreichend kleine Feldstärken und für isotrope Medien ist die Polarisation proportional zur elektrischen Feldstärke:

$$
\vec{P}=\chi \vec{E}
$$

Die skalare Proprotionalitätskonstante $\chi$ wird elektrische Suszeptibilität genannt. Die Polarisation des Mediums verstärkt im allgemeinen das elektrische Feld und führt zur elektrischen Verschiebungsdichte [35]:

$$
\vec{D}=\vec{E}+\frac{1}{\epsilon_{0}} \vec{P}
$$

Nutzt man die Proportionalität (4.4) aus, wird Gleichung (4.5) zu:

$$
\vec{D}=\vec{E}+\frac{1}{\epsilon_{0}} \vec{P}=\vec{E}+\frac{1}{\epsilon_{0}} \chi \vec{E}=\left(1+\frac{1}{\epsilon_{0}} \chi\right) \vec{E}=\epsilon \vec{E} .
$$

Der Vorfaktor wird zu einer einzigen Größe zusammengefaßt, der Dielektrizitätszahl:

$$
\epsilon=1+\frac{1}{\epsilon_{0}} \chi
$$

Sind die Felder zeitabhängig wie in (4.1) und (4.2), dann werden die Größen $\epsilon, \chi$ frequenzabhängig. Der Zusammenhang zwischen der makroskopischen Polarisation $\vec{P}$ und dem äußeren elektrischen Feld im statischen Fall $(\omega=0)$ ist

$$
\vec{P}=\epsilon_{0} \cdot(\epsilon(0)-1) \cdot \vec{E}
$$

Da die Polarisation bei harmonisch zeitabhängigen, äußeren, elektrischen Feld $(\vec{E}(t)$ $\left.=\vec{E}_{0} e^{i \omega t}\right)$ nicht in Phase mit diesem sein muß, wird dies in (4.8) durch die Einführung einer komplexen Dielektrizitätszahl berücksichtigt [104]:

$$
\begin{gathered}
\epsilon(\omega)=\epsilon^{\prime}(\omega)-i \epsilon^{\prime \prime}(\omega), \\
\tan \delta_{\epsilon}=\frac{\epsilon^{\prime \prime}}{\epsilon^{\prime}} .
\end{gathered}
$$

Der hier eingeführte dielektrische Verlustwinkel $\delta_{\epsilon}$ ist ein Maß für die Abgabe der Energie des externen elektrischen Feldes an die Flüssigkeit. Mit (4.9) wird (4.8) zu

$$
\vec{P}(t)=\epsilon_{0} \cdot(\epsilon(\omega)-1) \cdot \vec{E}(t) .
$$

Auch durch thermische Bewegungen der Moleküle treten Fluktuationen in der makroskopischen Polarisation des Mediums auf [83], da die üblichen Feldstärken von 
angelegten Feldern im Vergleich zu den Feldstärken im Molekularbereich vergleichsweise gering sind. Die Informationen über das Zeitverhalten, die in diesen Fluktuationen enthalten sind, werden prinzipiell durch die normierte Autokorrelationsfunktion der makroskopischen Polarisation zugänglich:

$$
\hat{\Phi}(t)=\frac{<\bar{P}\left(t+t_{0}\right) \cdot \bar{P}\left(t_{0}\right)>}{<\bar{P}\left(t_{0}\right) \cdot \bar{P}\left(t_{0}\right)>} .
$$

Jedoch sind die thermisch induzierten Rauschamplituden sehr klein, so daß sie meßtechnisch nicht zu erfassen sind. Deshalb führt man dem Medium von außen eine geringfügige Störung mittels eines elektrischen Feldes zu und beobachtet das Abklingen der Einstellung der Moleküle auf diese Störung. Ist das System linear, ist $\hat{\Phi}(t)$ gleich der Stufenantwortfunktion [121].

Wird also ein Medium im Zeitraum $t<t_{0}$ polarisiert und zum Zeitpunkt $t=t_{0}$ wieder sich selbst überlassen, beschreibt $\hat{\Phi}(t)$ das Abklingen der Polarisation. Der zeitliche Verlauf der Polarisation $\vec{P}(t)$ ist durch

$$
\vec{P}(t)=\vec{P}\left(t_{0}\right) \cdot \hat{\Phi}(t)
$$

gegeben. Ist das angelegte Feld von der Art (4.1), ergibt sich für die Polarisation

$$
\vec{P}(t)=\epsilon_{0}(\epsilon(0)-1) \cdot \int_{0}^{\infty} \vec{E}\left(t-t^{\prime}\right) \cdot\left(-\frac{\partial \hat{\Phi}}{\partial t^{\prime}}\right) d t^{\prime} .
$$

Setzt man (4.1) ein, läßt sich das Integral in (4.14) als Laplace-Transformation schreiben:

$$
\vec{P}(t)=\epsilon_{0}(\epsilon(0)-1) \cdot \vec{E}(t) \cdot \mathcal{L}_{i \omega}\left(-\hat{\Phi}\left(t^{\prime}\right)\right),
$$

und mit (4.8) erhält man

$$
\epsilon(\omega)=1+(\epsilon(0))-1) \cdot \mathcal{L}_{i \omega}\left(-\hat{\Phi}\left(t^{\prime}\right)\right)
$$

Im folgenden interessiert man sich nur für die Orientierungspolarisation und faßt den Beitrag der Verschiebungspolarisation in einer konstanten (in dem betrachteten Frequenzbereich frequenzunabhängigen) Größe $\epsilon_{\infty}$ zusammen. Es folgt schließlich

$$
\left.\epsilon(\omega)=\epsilon_{\infty}+(\epsilon(0))-\epsilon_{\infty}\right) \cdot \mathcal{L}_{i \omega}\left(-\hat{\Phi}\left(t^{\prime}\right)\right)
$$

\subsection{Relaxationsmodelle}

Die nun im weiteren betrachteten Relaxationsmodelle [104] beschreiben alle molekulare Vorgänge bzw. Relaxationen in einer Flüssigkeit im Frequenzbereich unterhalb von $100 \mathrm{GHz}$. Die schon erwähnte Verschiebungspolarisation spielt für das Relaxationsverhalten nur eine untergeordnete Rolle, da sie nur außerhalb des betrachteten Frequenzbereiches eine Frequenzabhängigkeit aufweist. 


\subsubsection{Die Debye-Funktion}

Das einfachste Modell einer solchen dielektrischen Abklingfunktion ist der exponentielle Abfall der Polarisation nach dem Abschalten des äußeren elektrischen Feldes $(t>1 p s)[52]$ ( $\Phi$ wird im folgenden für den Orientierungspolarisationsbeitrag verwandt):

$$
\Phi(t)=\Phi(0) \exp \left(-\frac{t}{\tau}\right)
$$

Die charakteristische Abklingzeit $\tau$ wird als Relaxationszeit bezeichnet. Mit (4.16) läßt sich die komplexe Dielektrizitätszahl berechnen; wenn man den Anteil der Verschiebungspolarisation $\epsilon_{\infty}$ dazu nimmt, ergibt sich

$$
\epsilon(\omega)=\epsilon_{\infty}+\frac{\epsilon(0)-\epsilon_{\infty}}{1+i \omega \tau} .
$$

Die daraus gewonnene Funktion wird als Debye-Funktion bezeichnet [39]. Zum besseren Verständnis betrachtet man die Aufteilung in Real- und Imaginärteil:

$$
\epsilon^{\prime}(\omega)=\frac{\epsilon(0)-\epsilon_{\infty}}{1+\omega^{2} \tau^{2}}+\epsilon_{\infty} \quad, \quad \epsilon^{\prime \prime}(\omega)=\frac{\left(\epsilon(0)-\epsilon_{\infty}\right) \omega \tau}{1+\omega^{2} \tau^{2}}
$$

\subsubsection{Die Cole-Cole-Funktion}

Faßt man das Abklingverhalten der Polarisation allgemeiner und nimmt man anstatt eines einzelnen exponentiellen Abfalles eine Verteilung von verschiedenen Relaxationszeiten an, so läßt sich die Stufenantwortfunktion, unter der Annahme, daß die Zeiten die Verteilung $G(\tau)$ besitzen, wie folgt darstellen:

$$
\Phi(t)=\int_{0}^{\infty} \exp \left(-\frac{t}{\tau}\right) G(\tau) d(\ln \tau),
$$

wobei die Gewichtsfunktion G durch

$$
\int_{0}^{\infty} G(\tau) d \tau=1
$$

normiert ist. K. S. Cole und R. H. Cole haben eine empirische Relaxationszeitverteilung gefunden [15]:

$$
\tau \cdot G(\tau)=\frac{\sin \pi \alpha}{2 \pi \cdot \cosh \left((1-\alpha) \ln \left(\frac{\tau_{0}}{\tau}\right)\right)-\cos (\pi \alpha)}, \quad \text { mit } 0 \leq \alpha<1 .
$$

Mit (4.16) und (4.21) ist es nun möglich, die komplexe Dielektrizitätszahl mit einer solchen Relaxationszeitverteilung zu beschreiben:

$$
\epsilon(\omega)=\epsilon_{\infty}+\frac{\epsilon(0)-\epsilon_{\infty}}{1+(i \omega \tau)^{1-\alpha}}
$$

Die Funktion wird als Cole-Cole-Spektralfunktion bezeichnet. Man sieht leicht, daß sie sich nur formal durch den Exponenten $1-\alpha$ von der Debye-Funktion unterscheidet. Der Parameter $\alpha(0 \leq \alpha<1)$ gibt die Breite der Relaxationszeitverteilung an. 
Für den Grenzfall $\alpha \rightarrow 0$ geht man erneut zu einer einzelnen Relaxationszeit über und Gleichung (4.24) wird zur Debye-Funktion.

\subsection{Reflexionsfaktormeßplatz}

Der Meßaufbau, im wesentlichen bestehend aus dem Netzwerkanalysator HP 8753 A und einer Reflexionszelle [47], besitzt einen Frequenzbereich von $300 \mathrm{kHz}$ bis 3 GHz. Der komplette Meßaufbau ist in Abb. 4.1 dargestellt.

Der Netzwerkanalysator erzeugt ein Signal am Ausgang (A). Im Leistungsteiler (3) wird das Signal in das Meßsignal und Referenzsignal, welches zurück in den Eingang (R) des Netzwerkanalysators läuft, aufgeteilt. Anschließend wird das Meßsignal in der CutOff-Meßzelle (5) reflektiert, so daß es den Richtkoppler (4) erreicht. Dort wird es aus der ursprünglichen Leitung ausgekop-

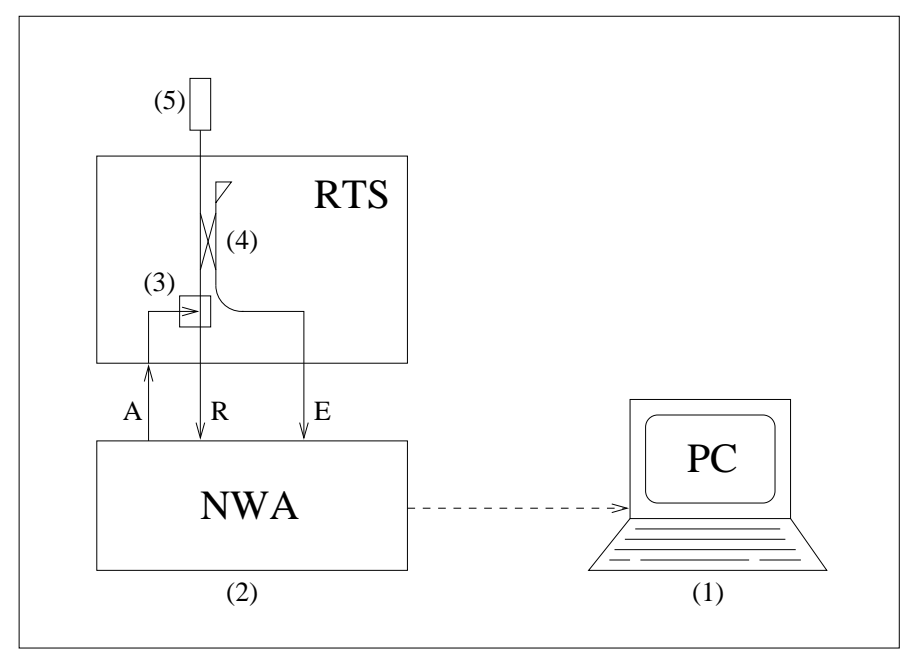

Abb. 4.1: Schematischer Aufbau des NetzwerkanalysatorMeßplatzes pelt und gelangt schließlich an den Meßeingang (E) des Netzwerkanalysators. Der Netzwerkanalysator vergleicht die Signale am Referenzeingang (A) und Meßeingang (E) nach Betrag und Phasendifferenz und errechnet so den komplexen Reflexionsfaktor r. Der Meßablauf wird von einem Computerprogramm gesteuert.

Der Reflexionsfaktor wird bei 51 fest voreingestellten Frequenzen bestimmt und nach einer erfolgreichen Messung über eine IEEE 488 Schnittstelle an den Computer (1) übermittelt. Leistungsteiler (3) und Richtkoppler (4) sind im Reflexionstestset (RTS) HP 85044A zusammengefaßt.

Da zwischen Netzwerkanalysator und Meßzelle das Reflexionstestset geschaltet ist, und da diese drei Komponenten mit flexiblen koaxialen Zuleitungen untereinander verbunden sind, ist vor jeder Messung eine Kalibration bezüglich der Eingangsebene der Meßzelle nötig. Das Relfexion-Test-Set in Abb. 4.1 kann als ein Sechspol betrachtet werden, dessen Übertragungseigenschaften durch eine Streumatrix beschrieben werden. Die Parameter dieser Matrix werden bei der Kalibration bestimmt, indem der Reflexionsfaktor $\mathrm{r}$ bei drei verschiedenen Kalibrierabschlüssen (Kurzschluß, offenes Ende, angepaßter Abschluß) gemessen wird.

\subsubsection{Die Meßzelle vom Typ 1}

Bei der Cut-Off-Meßzelle vom Typ 1 wird das Signal über die Koaxialleitung (1) durch ein Teflonfenster (2) zur Flüssigkeit geführt (Abb. 4.2). Das Teflonfenster ist durch einen erweiterten Außenleiter und durch einen verengten Innenleiter an den 
Wellenwiderstand $Z_{0}$ von $50 \Omega$ der Koaxialleitung angepaßt, um störende Reflexionen schon an dieser Stelle zu vermeiden.

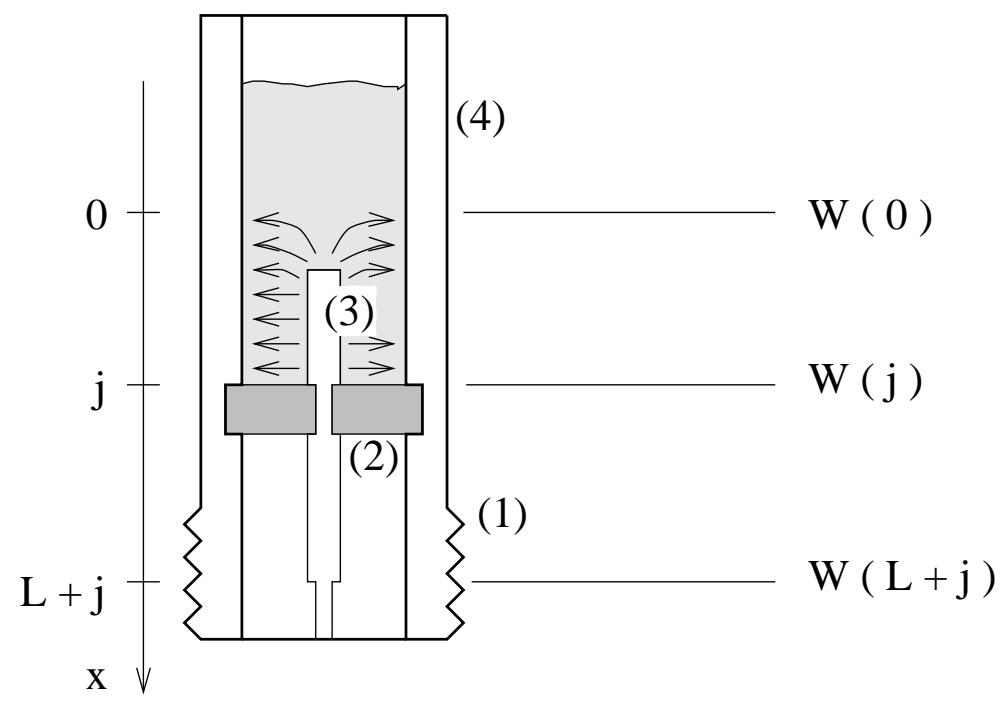

Abb. 4.2: Cut-Off-Meßzelle Typ 1

Der bekannte Abschlußwiderstand $W(0) \rightarrow \infty$ an einer Stelle mit zunächst nicht bekanntem Abstand vom Fenster muß nun mit Hilfe der Widerstandstransformation [104] in zwei Schritten in die Kalibrationsebene transformiert werden. Zunächst wird von der Reflexionsebene bei $\mathrm{x}=0$ in die Ebene Flüssigkeit/Teflon bei j transformiert:

$$
W(j)=Z_{j} \frac{W(0)+Z_{j} \tanh \left(j \gamma_{j}\right)}{W(0) \tanh \left(j \gamma_{j}\right)+Z_{j}}
$$

dabei ist $Z_{j}$ der Wellenwiderstand des mit der Flüssigkeit angefüllten Koaxialstückes (3)

$$
Z_{j}(\omega)=\frac{Z_{0}}{\sqrt{\epsilon(\omega)}},
$$

und $\gamma_{j}$ bezeichnet den Ausbreitungsexponenten

$$
\gamma_{j}(\omega)=\frac{i \omega}{c_{0}} \sqrt{\epsilon(\omega)}
$$

Da die Meßzelle im Cut-Off-Modus betrieben wird, geht $W(0) \rightarrow \infty$. Daher läßt sich (4.25) vereinfachen:

$$
W(j)=Z_{j} \frac{1}{\tanh \left(j \gamma_{j}\right)} .
$$

Weiter muß dieses Zwischenergebnis in die Kalibrationsebene bei $\mathrm{L}+\mathrm{j}$ transformiert werden, für die der Netzwerkanalysator geeicht wird:

$$
W(L+j)=Z_{j} \frac{W(j)+Z_{0} \tanh \left(L \gamma_{0}\right)}{W(j) \tanh \left(L \gamma_{0}\right)+Z_{0}}
$$

$Z_{0}$ ist der Wellenwiderstand der Koaxialleitung und $\gamma_{0}$ der Ausbreitungskoeffizient: 


$$
\gamma_{0}(\omega)=\frac{i \omega}{c_{0}}
$$

Der Netzwerkanalysator mißt, wie oben beschrieben, den Reflexionsfaktor r. Dieser läßt sich durch den Wellenwiderstand $Z_{0}$ und das Transformationsergebnis $W(L+j)$ ausdrücken:

$$
r=\frac{W(L+j)-Z_{0}}{W(L+j)+Z_{0}}
$$

Mit (4.28) und (4.29) ergibt das:

$$
r=\frac{1-\tanh \left(L \gamma_{0}\right)}{1+\tanh \left(L \gamma_{0}\right)} \cdot \frac{1-\sqrt{\epsilon} \tanh \left(j \gamma_{0} \sqrt{\epsilon(\omega)}\right)}{1+\sqrt{\epsilon} \tanh \left(j \gamma_{0} \sqrt{\epsilon(\omega)}\right)} .
$$

Die beiden unbekannten Größen L und j werden durch eine Referenzmessung mit Wasser bestimmt. Dazu wird der Reflexionsfaktor $\mathrm{r}$ von doppelt destilliertem und deionisiertem Wasser bei $25^{\circ}$ bei allen Frequenzen gemessen. Die Dielektrizitätszahl $\epsilon(\omega)$ der Referenzflüssigkeit wird dabei durch eine Debye-Funktion mit den Parametern

$$
\begin{aligned}
\epsilon(0) & =78.36 \\
\epsilon_{\infty} & =5.1 \\
\tau & =8.27 p s \text { und } \\
\sigma & =0.0004 \frac{S}{m}
\end{aligned}
$$

beschrieben [73]. Unter Variation von L und $\mathrm{j}$ werden die theoretisch berechneten Werte $r_{\text {theo }}$ nach einem Verfahren von Marquart (Least-Square-Algorithmus) [98] an die real gemessenen Reflexionsfaktoren $r_{\text {mess }}$ angepaßt und daraus L und j bestimmt.

\subsubsection{Die Meßzelle vom Typ 2}

Der Unterschied von Meßzelle 2 zu Meßzelle 1 liegt in einem nicht angepaßten Teflonfenster. Innen- und Außenleiter sind hier nicht an den $50 \Omega$ Wellenwiderstand der Zuleitung angepaßt. Ein Teil des Signals wird aufgrund des Impedanzsprunges an der Grenzfläche von der Luft zum Teflonfenster schon bei $\mathrm{x}=\mathrm{T}+\mathrm{j}$ reflektiert. Ein weiterer Teil des transmittierten Signals wird wie bei der Zelle vom Typ 1 zusätzlich an der Grenzfläche vom Teflon zur Flüssigkeit reflektiert, da auch hier ein Impedanzsprung vorliegt. Der schließlich in die Flüssigkeit transmittierte Teil des Signals wird durch die Cut-Off-Eigenschaften wegen $W(0) \rightarrow \infty$ dort reflektiert.

Die Transformation von W(0) erfolgt in analoger Weise zur Behandlung zur Meßzelle 1 in zwei Schritten.

$$
W(T+j)=Z_{T} \frac{W(j)+Z_{T} \tanh \left(L \gamma_{T}\right)}{W(j) \tanh \left(L \gamma_{T}\right)+Z_{T}} .
$$

Dabei ist $Z_{T}$ der Wellenwiderstand im Teflon angefüllten Koaxialstück (2) mit der Dielektrizitätszahl $\epsilon_{T}=2.1$ 


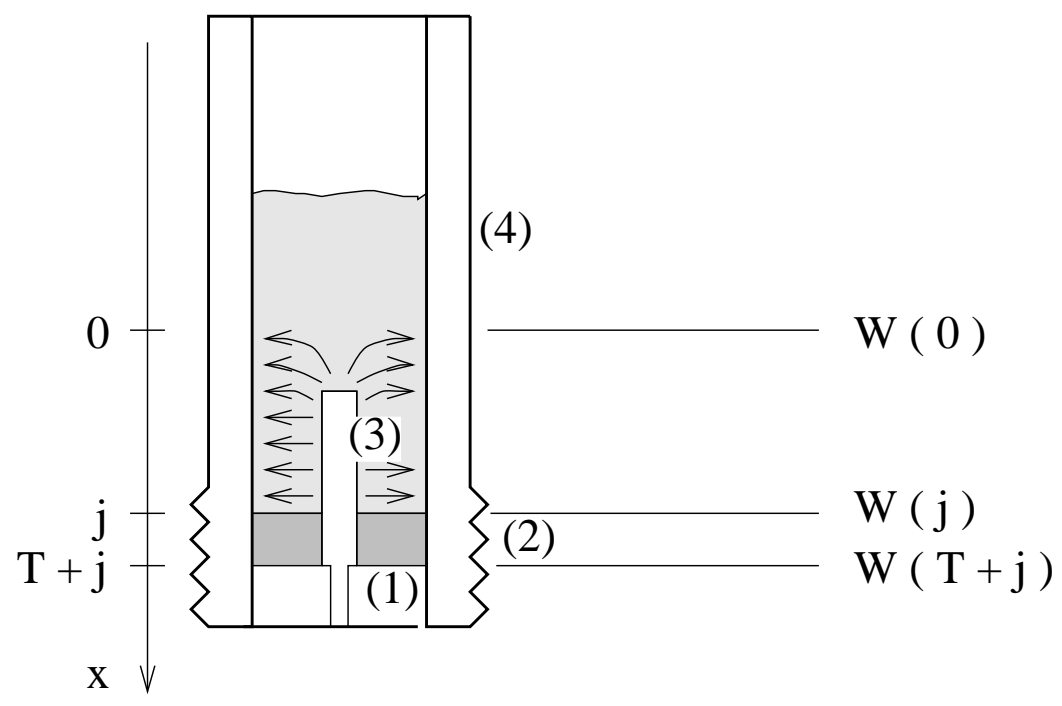

Abb. 4.3: Cut-Off-Meßzelle Typ 2

$$
Z_{T}(\omega)=\frac{Z_{0}}{\sqrt{\epsilon_{T}}}
$$

$\gamma_{T}$ bezeichnet den Ausbreitungsexponenten im Teflonstück:

$$
\gamma_{T}(\omega)=\frac{i \omega}{c_{0}} \sqrt{\epsilon_{T}}
$$

In analoger Rechnung zur Zelle vom Typ 1 erhält man den Reflexionsfaktor:

$$
r=\frac{1+\frac{\sqrt{\epsilon}}{\sqrt{\epsilon_{T}}} \tanh \left(\sqrt{\epsilon} \gamma_{0} j\right) \tanh \left(\gamma_{T} T\right)-\sqrt{\epsilon} \tanh \left(\sqrt{\epsilon} \gamma_{0} j\right)-\sqrt{\epsilon_{T}} \tanh \left(\gamma_{T} T\right)}{1+\frac{\sqrt{\epsilon}}{\sqrt{\epsilon_{T}}} \tanh \left(\sqrt{\epsilon} \gamma_{0} j\right) \tanh \left(\gamma_{T} T\right)+\sqrt{\epsilon} \tanh \left(\sqrt{\epsilon} \gamma_{0} j\right)+\sqrt{\epsilon_{T}} \tanh \left(\gamma_{T} T\right)}
$$

Die Meßzelle des Typs 2 hat den entscheidenden Vorteil, daß wegen des nicht angepaßten Teflonfensters keine Inhomogenitäten des elektrischen Feldes auftreten. Dagegen kommt es insbesondere bei hohen Frequenzen durch die verschiedenen Radien der Zuleitung und des Teflonfensters bei der Meßzelle vom Typ 1 zu Inhomogenitäten im elektrischen Feld. Diese führt zu Meßfehlern, die dann durch eine geeignet geänderte Modellierung der Zelle durch Berücksichtigung von Zusatzkapazitäten korrigiert werden müssen [117]. Die Bestimmung der Größen L und j erfolgt in analoger Weise wie bei der Meßzelle von Typ 1.

\subsubsection{Fehlerabschätzung}

Die Messungen für hohe Frequenzen (>20 MHz) werden mit der Zelle vom Typ 2 und einer geometrischen Innenleiterlänge von Null und im tieffrequenten Bereich $(<20 \mathrm{MHz})$ mit der Zelle vom Typ 1 und einer geometrischen Innenleiterlänge von 16mm durchgeführt. Anschließend werden die gemessenen Spektren dann zu einem einzigen zusammengefügt.

Die Fehler der Meßwerte sind in der folgenden Tabelle aufgeführt. 


\begin{tabular}{|l|l|l|}
\hline Frequenzbereich & Fehler von $\epsilon^{\prime}$ & Fehler von $\epsilon^{\prime \prime}$ \\
\hline \hline $300 \mathrm{kHz}-800 \mathrm{kHz}$ & $1,5 \%$ & $2 \%$ \\
$800 \mathrm{kHz}-1 \mathrm{GHz}$ & $0,2 \%$ & $1 \%$ \\
$1 \mathrm{GHz}-3 \mathrm{GHz}$ & $2,5 \%$ & $5 \%$ \\
\hline
\end{tabular}

Tabelle 4.1: Fehler des Netzwerkanalysators in den verschiedenen Meßbereichen

\subsection{Die Spektren}

Im folgenden werden zunächst die Spektren der Lipidlösungen vorgestellt. Die aufgenommenen Spektren der verschiedenen Suspensionen zeigen untereinander einen höchst unterschiedlichen Verlauf. Da der Effekt, der durch die Umorientierung der Kopfgruppen hervorgerufen wird, relativ gering ist, wurde zunächst eine Konzentrationsreihe von reinem DMPC aufgenommen, um somit den Effekt eindeutig der Kopfgruppenumorientierung zuordnen zu können. Aus Gründen der Vereinfachung wird in den folgenden Abbildungen nur der Realteil $\epsilon^{\prime}$ dargestellt.

Es stellte sich bei den ersten Messungen heraus, daß eine Konzentration von 60 $\mathrm{mg} / \mathrm{ml}$ ausreichend hoch ist, um eine genügend gute Genauigkeit zu gewährleisten. Somit wurde diese Konzentration bei allen Lipidmischungen verwendet.

Problematisch ist im Zusammenhang mit den dielektrischen Messungen das Vorhandensein von freien Ionen in der Lösung bzw. Gegenionen auf der Oberfläche der Vesikel. Freie Ionen können mittels des Maxwell-Wagner-Effektes zu einem lokal relativ hohen Dipolmoment innerhalb der Flüssigkeit führen, was sich in einer entsprechend hohen Polarisierbarkeit und damit in einer hohen Dielektrizitätszahl niederschlägt. Gegenionen können durch Bewegungen an der Oberfläche und die Änderung ihrer Konzentration auf der Oberfläche der Vesikel ebenfalls zu einer starken Beeinflussung der Spektren beitragen. In [131] wird dieser Effekte ausführlich mittels einer entsprechenden Theorie von C. Grosse [45] behandelt und soll hier nicht weiter betrachtet werden. Jedoch sind diese Effekte möglicherweise derart stark, daß der Einfluß der Kopfgruppenumlagerung fast völlig verdeckt wird. Solche Ionen können durch Verunreinigungen des Lösungsmittels, durch die Extrusionsprozedur oder durch Verunreinigungen des trockenen Lipidpulvers selbst, mittels entsprechender Isomere, in die Lösung gelangen. Beide zuerst genannten Faktoren können hier jedoch weitestgehends ausgeschlossen werden. Das verwendete Wasser hat eine Leitfähigkeit von nur 0,0004 $\frac{S}{m}$ und der Extruder wird vor und nach jeder Benutzung gründlich gereinigt. Viel wahrscheinlicher ist eine Verunreinigung durch ionische Lipide oder Fettsäurereste. Zum Vergleich der vom Hersteller angegebenen Reinheitsgrade siehe Kapitel 2.6.1.

\subsubsection{DMPC}

In Abb. 4.4 ist eine Konzentrationsreihe von reinem DMPC/Wasser bei $30^{\circ} \mathrm{C}$ dargestellt, jedoch nur die Meßdaten der hochfrequenten Meßzelle von $2 \mathrm{kHz}$ bis $2 \mathrm{GHz}$. Es ist zum besseren Vergleich der Relaxationsstufe $\epsilon^{\prime}-\delta \epsilon_{\text {Wasser }}\left(\delta \epsilon_{\text {Wasser }}=\delta \epsilon_{3}\right.$ aus Gleichung (4.37)) aufgetragen. Deutlich ist mit höherer Konzentration ein starkes Ansteigen einer Relaxationsstufe bei ca. $60 \mathrm{MHz}$ zu sehen. Allerdings zeigt sich erst 


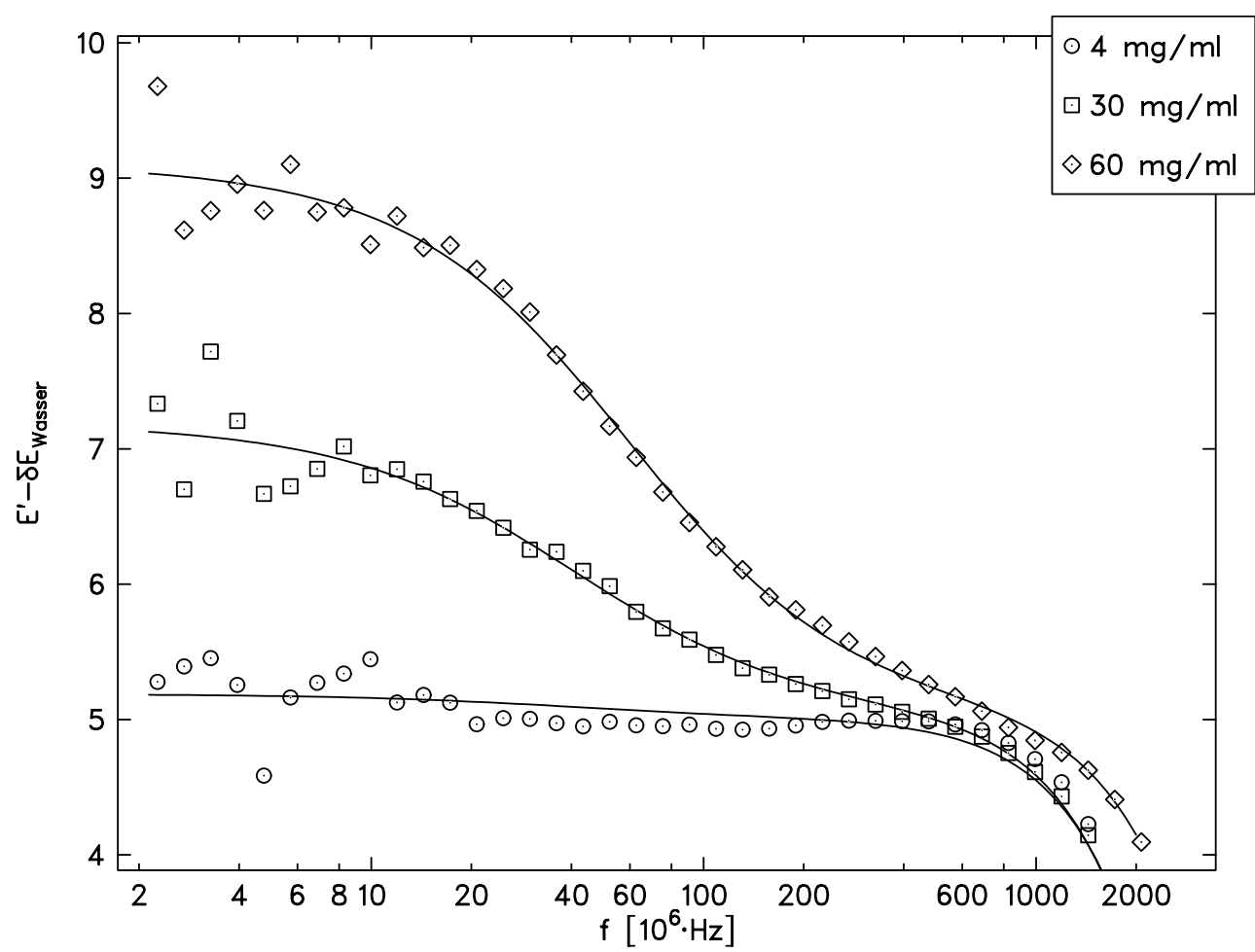

Abb. 4.4: Realteil des dielektrischen Spektrums drei verschieden konzentrierter DMPC/Wasser Suspensionen bei $30{ }^{\circ} \mathrm{C}$ mit angepaßter Spektralfunktion abzüglich der Wasserrelaxationsstufe $\left(\delta \epsilon_{\text {Wasser }}=\right.$ $\delta \epsilon_{3}$ aus Gleichung (4.37); dargestellt sind hier nur die Daten der tieffrequenten Meßzelle

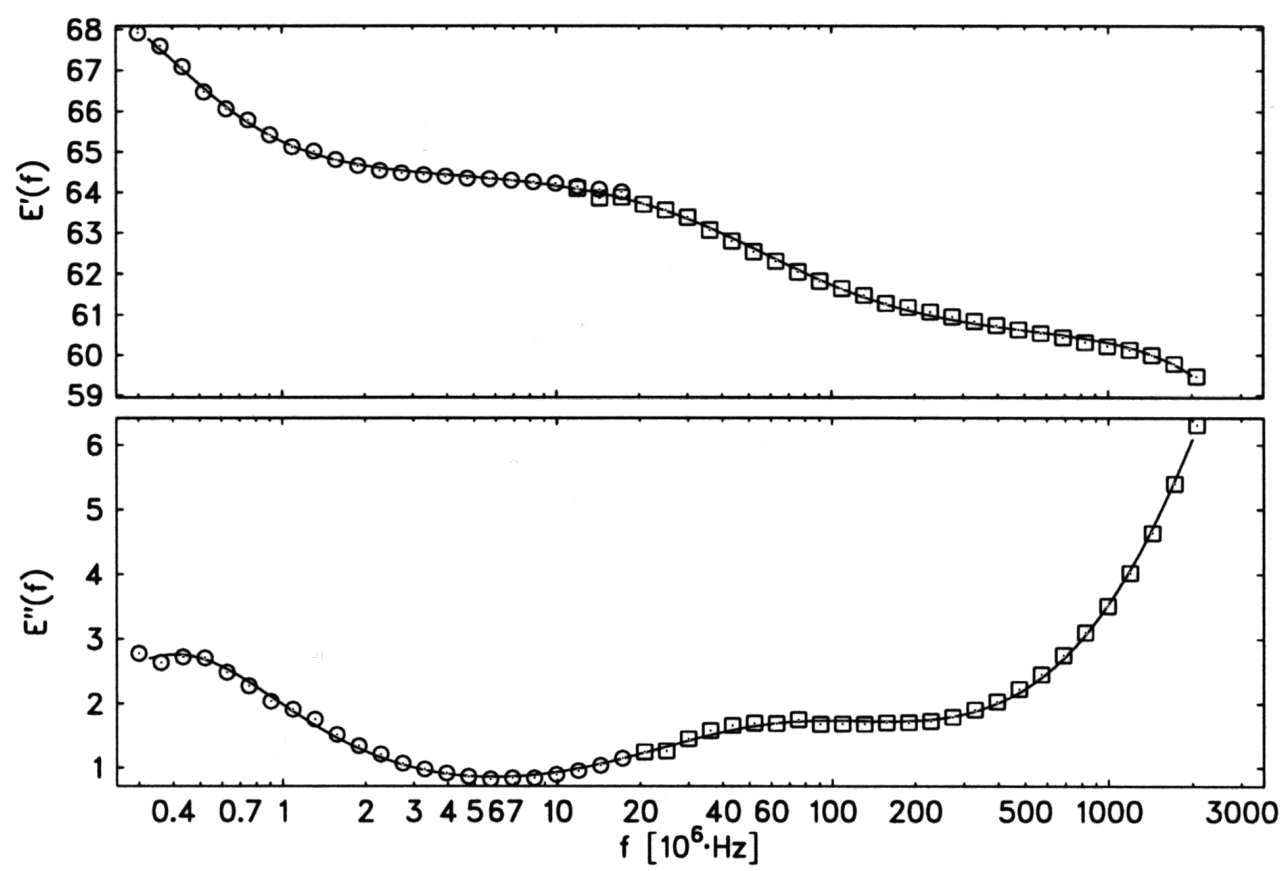

Abb. 4.5: Dielektrisches Spektrum der DMPC/Wasser Suspensionen $60 \mathrm{mg} / \mathrm{ml}$ bei $31{ }^{\circ} \mathrm{C}$ mit angepaßter Spektralfunktion Gleichung (4.37); oben: $\epsilon^{\prime}(f)$, unten: $\epsilon^{\prime \prime}(f)$ ohne Leitfähigkeitsanteil; $\bigcirc$ : Daten der tieffrequenten Meßzelle; $\square$ : hochfrequente Meßzelle 


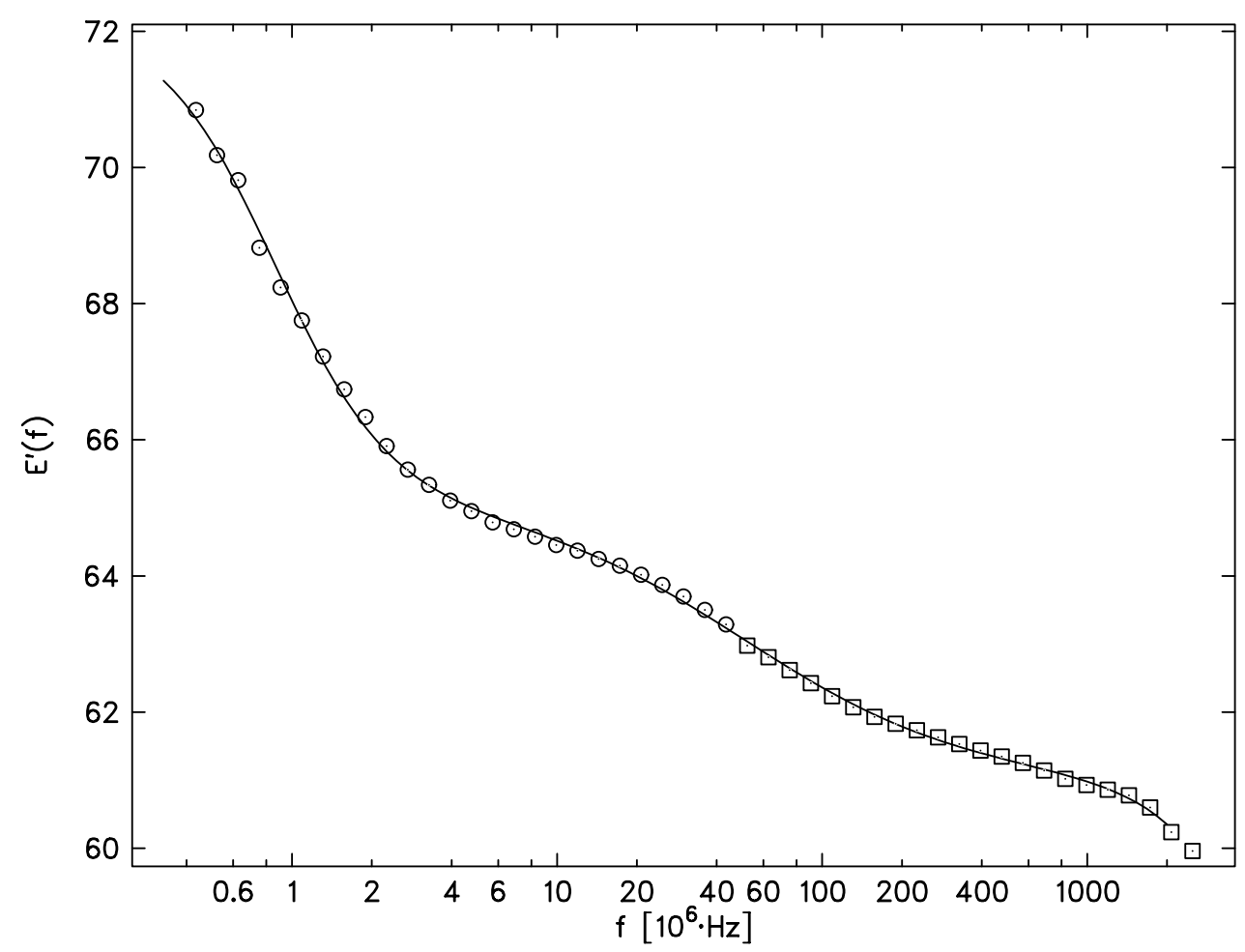

Abb. 4.6: Realteil des dielektrischen Spektrums der DMPC/Cholesterin 85:15 Suspensionen bei $31{ }^{\circ} \mathrm{C}$ mit angepaßter Spektralfunktion Gleichung (4.37)

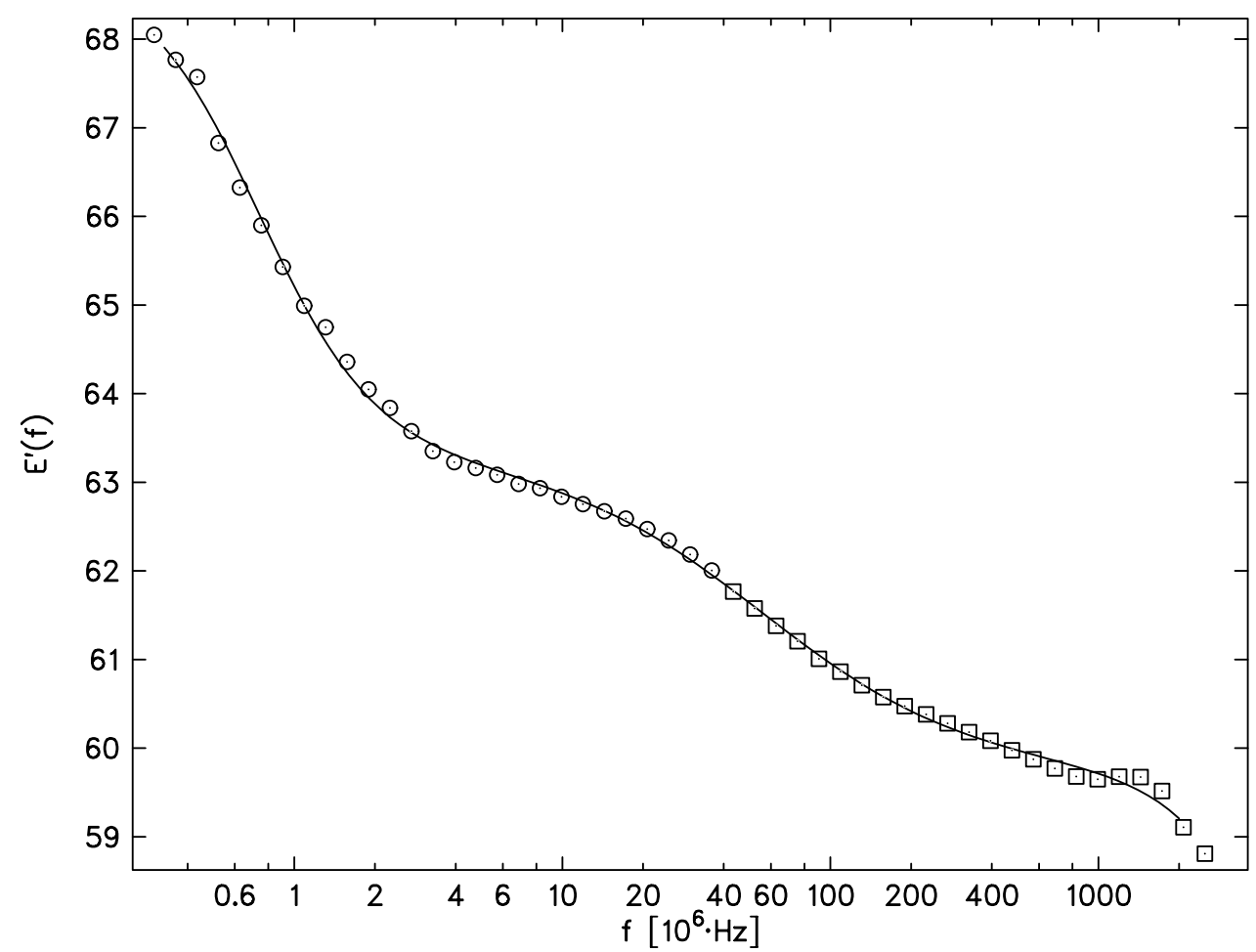

Abb. 4.7: Realteil des dielektrischen Spektrums der DMPC/Cholesterin 70:30 Suspensionen bei $31{ }^{\circ} \mathrm{C}$ mit angepaßter Spektralfunktion Gleichung (4.37) 
bei vergleichsweise sehr hohen Lipidkonzentrationen ein definitiv meßbarer Effekt. Abb. 4.5 zeigt nun ein Spektrum von reinem DMPC bei $31^{\circ} \mathrm{C}$, welches sich aus den Meßdaten beider Meßzellen zusammensetzt. Im Bereich von etwa 10 bis $20 \mathrm{MHz}$ wurde mit beiden Zellen gemessen. Deutlich ist ein stufenförmiger Abfall von $\epsilon^{\prime}$ mit steigender Frequenz zu beobachten. Insgesamt können die Spektren der DMPC/Wasser Suspension unter Vorgabe von $\epsilon_{\infty}$ aus [73] durch einen tieffrequenten $(\nu \leq 2 \mathrm{MHz})$ Debye-Term, einen mittelfrequenten $(2 \mathrm{MHz} \leq \nu \leq 200 \mathrm{MHz})$ Cole-Cole-Term und einen hochfrequenten $(\nu \geq 200 \mathrm{MHz})$ Debye-Term beschrieben werden. Dabei ordnet man nun den tieffrequenten Relaxationsbeitrag der Diffusionsbewegung der Ionen in der Lösung um die Vesikel herum zu, den mittelfrequenten Beitrag der Kopfgruppenumlagerung und den hochfrequenten Beitrag der Wasserrelaxation. Zusätzlich wird im Imaginärteil die Dielektrizitätszahl noch ein Leitfähigkeitsbeitrag berücksichtigt.

$$
\epsilon(\omega)=\epsilon_{\infty}+\frac{\delta \epsilon_{1}}{1+i \omega \tau_{1}}+\frac{\delta \epsilon_{2}}{1+\left(i \omega \tau_{2}\right)^{1-\alpha}}+\frac{\delta \epsilon_{3}}{1+i \omega \tau_{3}}-\frac{i \sigma(0)}{\epsilon_{0} \omega}
$$

Bis auf den tieffrequenten Debye-Term wird diese Spektralfunktion ebenfalls zur Anpassung bei der konzentrationsabhängigen Messung (Abb. 4.4) verwendet.

\subsubsection{DMPC/Cholesterin}

Die Spektren der Suspensionen DMPC/Cholesterin 85:15 und 70:30 zeigen qualitativ (oftmals auch quantitativ) den gleichen Verlauf, wie die Spektren von reinem DMPC. Allein der höhere tieffrequente Relaxationsbeitrag fällt im ersten Augenblick auf. Eine genauere Betrachtung der Ergebnisse aus den Anpaßrechnungen zeigt jedoch auch einen Unterschied im Temperaturverhalten des mittelfrequenten Relaxationsterms im Vergleich zu reinem DMPC und auch untereinander.

\subsubsection{DMPC/DPPC}

Die Anpassung der Spektralfunktion (4.37) an die Spektren der DMPC/DPPC 50:50 Suspension führt zu keinem zufriedenstellenden Ergebnis. Es zeigte sich jedoch, daß die Ersetzung des tieffrequenten Debye-Terms durch einen entsprechenden ColeCole-Term die gewünschte gute Beschreibung der Meßergebnisse ermöglicht. Somit wird folgende Spektralfunktion benutzt:

$$
\epsilon(\omega)=\epsilon_{\infty}+\frac{\delta \epsilon_{1}}{1+\left(i \omega \tau_{1}\right)^{1-\alpha_{1}}}+\frac{\delta \epsilon_{2}}{1+\left(i \omega \tau_{2}\right)^{1-\alpha_{2}}}+\frac{\delta \epsilon_{3}}{1+i \omega \tau_{3}}-\frac{i \sigma(0)}{\epsilon_{0} \omega}
$$

In Abb. 4.8 ist das Spektrum der DMPC/DPPC 50:50 dargestellt. Auffällig ist vor allem das im Vergleich zum Spektrum von DMPC sehr starke Ansteigen von $\epsilon^{\prime}(f)$ zu tiefen Frequenzen hin. Dieser Effekt wird dem verstärkten Vorhandensein von freien Ionen durch Verunreinigungen des DPPC durch Lipide mit Gegenionen zugeschrieben. Trotz der dadurch resultierenden hohen Leitfähigkeit kann noch immer die mittelfrequente Relaxationsstufe um $60 \mathrm{MHz}$, die der Kopfgruppenumlagerung zugeschrieben wird, ausgemacht werden. Betrachtet man jedoch die Spektren unterhalb von $32{ }^{\circ} \mathrm{C}$, so verlagert sich der mittelfrequente Relaxationsspektralterm derart stark zu tieferen Frequenzen, daß bei den Anpaßrechnungen die Amplitude 


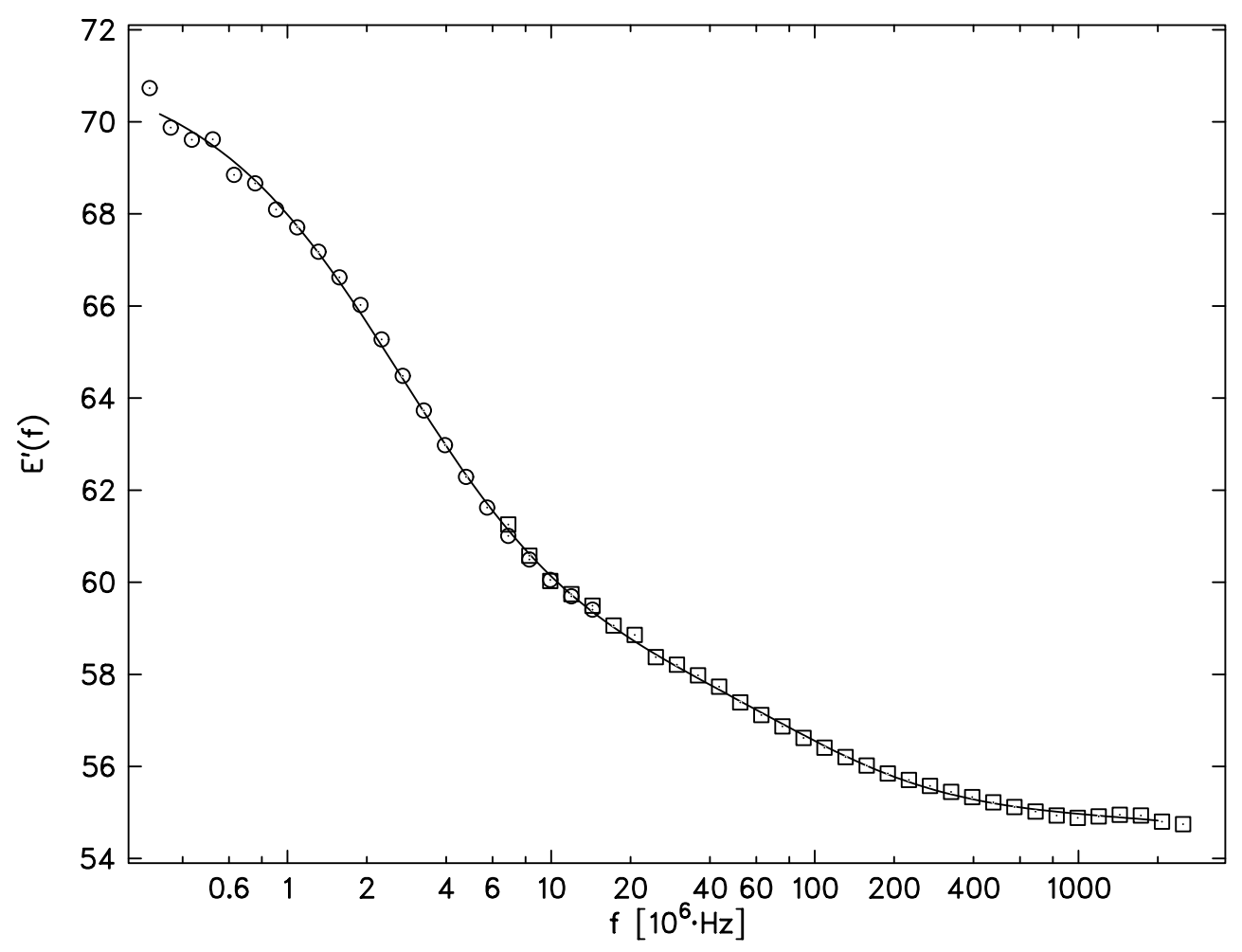

Abb. 4.8: Realteil des dielektrischen Spektrums der DMPC/DPPC 50:50 Suspensionen bei $40{ }^{\circ} \mathrm{C}$ mit angepaßter Spektralfunktion Gleichung (4.38)

$\delta \epsilon_{2}$ und der Verteilungsparameter $\alpha$ auf einem konstanten Wert festgehalten werden mussten. So können aber dennoch wenigstens die Relaxationszeiten einigermaßen sinnvoll ermittelt werden.

\subsubsection{DMPC/DMG}

Die Spektren der DMPC/DMG Suspension zeigen im tieffrequenten ein vollständig anderes Verhalten als die bisher vorgestellten Spektren. Alle Versuche den tieffrequenten Relaxationsbeitrag mit Hilfe eines Debye-Terms, eines Cole-Cole-Terms oder eines Davidson-Cole-Terms, eines Havriliak-Negami-Terms hinreichend zu beschreiben schlagen fehl. Erst die Verwendung von zwei tieffrequenten Debye-Termen bringt eine Anpaßgenauigkeit, wie sie bei den bisher behandelten Spektren gegeben ist.

$$
\epsilon(\omega)=\epsilon_{\infty}+\frac{\delta \epsilon_{1 a}}{1+i \omega \tau_{1 a}}+\frac{\delta \epsilon_{1 b}}{1+i \omega \tau_{1 b}}+\frac{\delta \epsilon_{2}}{1+\left(i \omega \tau_{2}\right)^{1-\alpha}}+\frac{\delta \epsilon_{3}}{1+i \omega \tau_{3}}-\frac{i \sigma(0)}{\epsilon_{0} \omega}
$$

In Abb. 4.9 und 4.10 ist beispielhaft ein Spektrum der DMPC/DMG Suspension bei $60{ }^{\circ} \mathrm{C}$ dargestellt. Deutlich ist ein vergleichsweise sehr starkes Ansteigen im tieffrequenten Bereich zu sehen, welches nur durch zwei Debye-Terme ausreichend beschrieben werden kann. Um die Relaxationsstufe der Kopfgruppenumlagerung deutlich zu machen, ist in Abb. 4.10 ein kleinerer Ausschnitt des Spektrums dargestellt. 


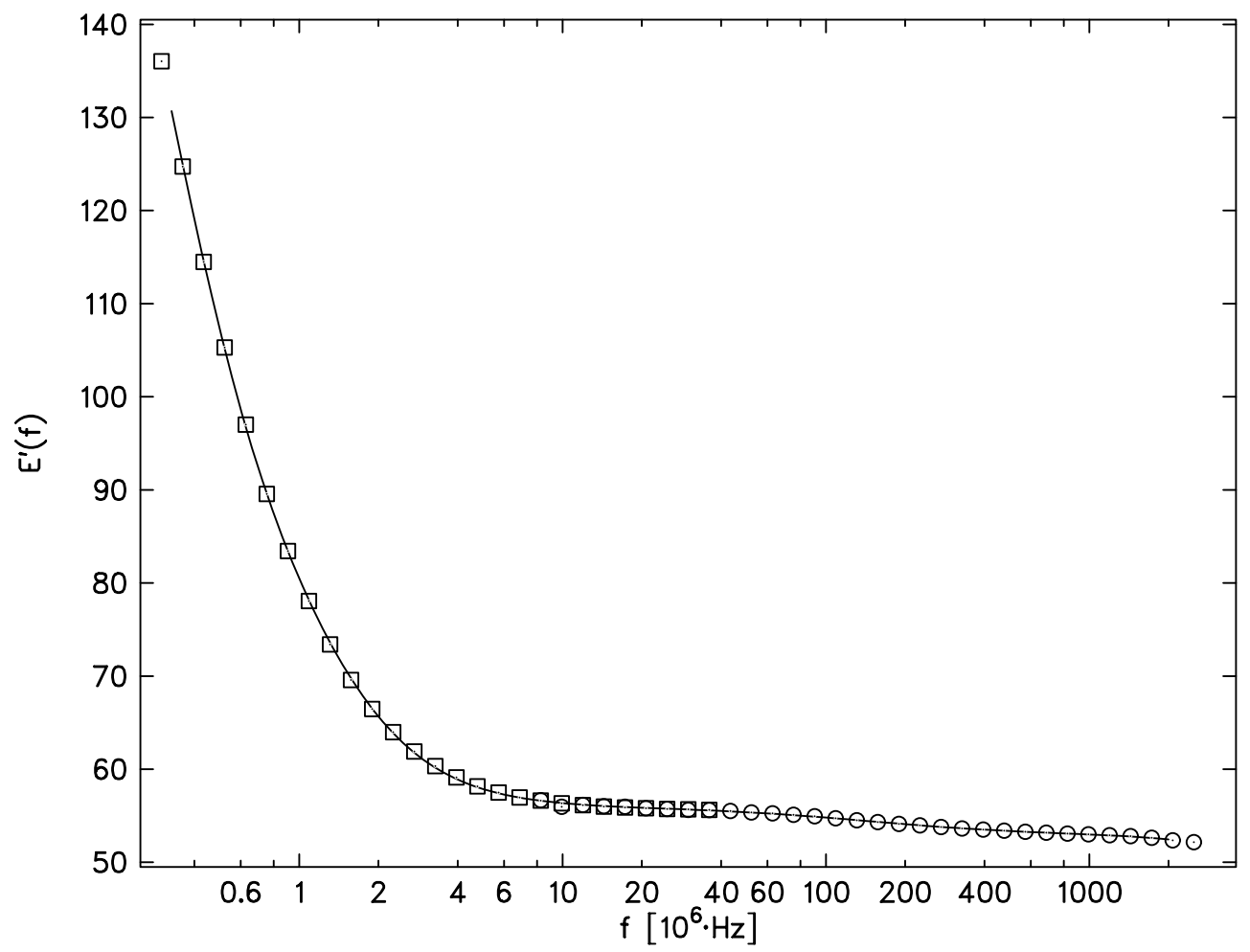

Abb. 4.9: Realteil des dielektrischen Spektrums der DMPC/DMG 75:25 Suspensionen bei $60{ }^{\circ} \mathrm{C}$ mit angepaßter Spektralfunktion Gleichung (4.39)

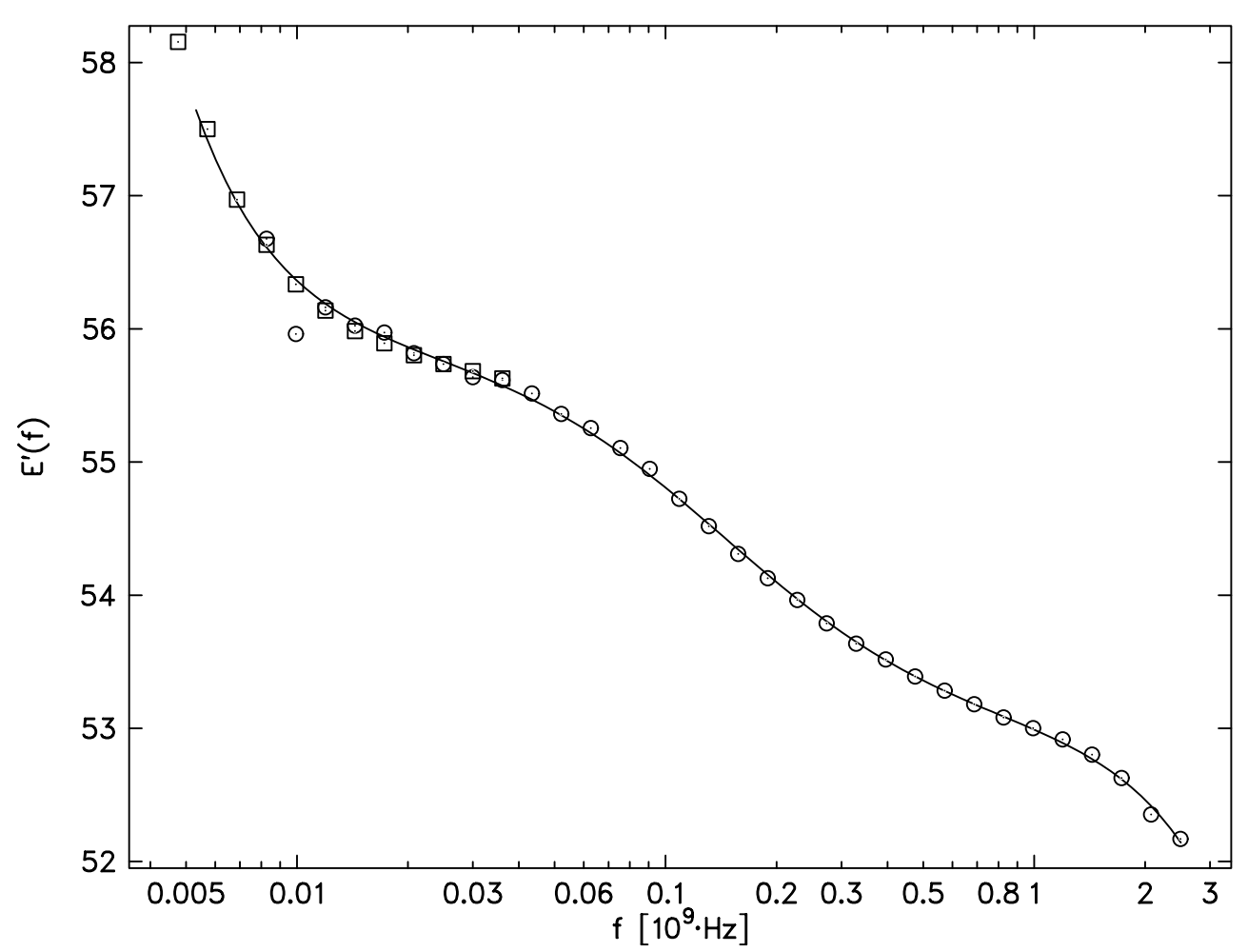

Abb. 4.10: Ausschnitt aus Abb. 4.9 der Suspension DMPC/DMG $75: 25$ bei $60{ }^{\circ} \mathrm{C}$ 
reines DMPC

\begin{tabular}{|c|c|c|c|c|c|c|c|c|}
\hline $\mathrm{T}\left[{ }^{\circ} C\right]$ & $\delta \epsilon_{3}$ & $\tau_{3}[\mathrm{~ns}]$ & $\delta \epsilon_{2}$ & $\tau_{2}[\mathrm{~ns}]$ & $\alpha$ & $\delta \epsilon_{1}$ & $\tau_{1}[\mathrm{~ns}]$ & $\sigma \frac{m S}{m}$ \\
\hline 18 & $63,13(3)$ & $10,52(5)$ & $5,2(1)$ & $12,7(6)$ & $0,39(1)$ & $33(21)$ & $2226(784)$ & $1,08(4)$ \\
21 & $62,48(4)$ & $10,25(5)$ & $4,9(2)$ & $11,2(8)$ & $0,37(2)$ & $0,14(14)$ & $535(636)$ & $1,31(3)$ \\
23 & $61,37(5)$ & $9,91(7)$ & $4,9(2)$ & $8,2(7)$ & $0,36(2)$ & $0,4(1)$ & $202(96)$ & $1,347(2)$ \\
23,5 & $60,55(4)$ & $9,77(6)$ & $4,6(1)$ & $6,2(3)$ & $0,31(2)$ & $3,0(3)$ & $400(39)$ & $1,313(3)$ \\
24 & $59,39(5)$ & $9,63(7)$ & $4,7(1)$ & $4,6(2)$ & $0,29(2)$ & $3,0(2)$ & $367(33)$ & $1,325(3)$ \\
24,5 & $58,99(4)$ & $9,56(7)$ & $4,61(8)$ & $3,9(1)$ & $0,25(1)$ & $4,3(4)$ & $485(42)$ & $1,346(4)$ \\
25 & $58,72(5)$ & $9,46(7)$ & $4,68(8)$ & $3,8(1)$ & $0,26(2)$ & $6,9(8)$ & $623(56)$ & $1,335(6)$ \\
26 & $58,43(4)$ & $9,39(7)$ & $4,45(7)$ & $3,35(9)$ & $0,23(1)$ & $4,9(4)$ & $488(37)$ & $1,382(4)$ \\
28 & $57,81(4)$ & $9,16(7)$ & $4,38(7)$ & $3,02(9)$ & $0,22(1)$ & $4,2(3)$ & $401(27)$ & $1,442(3)$ \\
31 & $56,92(4)$ & $8,80(7)$ & $4,17(7)$ & $2,58(8)$ & $0,20(2)$ & $5,6(4)$ & $435(26)$ & $1,516(3)$ \\
\hline
\end{tabular}

DMPC/Cholesterin 85:15

\begin{tabular}{|c|c|c|c|c|c|c|c|c|}
\hline $\mathrm{T}\left[{ }^{\circ} C\right]$ & $\delta \epsilon_{3}$ & $\tau_{3}[\mathrm{~ns}]$ & $\delta \epsilon_{2}$ & $\tau_{2}[\mathrm{~ns}]$ & $\alpha$ & $\delta \epsilon_{1}$ & $\tau_{1}[\mathrm{~ns}]$ & $\sigma \frac{m S}{m}$ \\
\hline 18 & $60,18(5)$ & $10,61(6)$ & $5,4(2)$ & $10,0(7)$ & $0,39(2)$ & $4,8(1)$ & $246(11)$ & $4,559(2)$ \\
21 & $59,30(5)$ & $10,16(6)$ & $5,0(2)$ & $6,8(4)$ & $0,37(2)$ & $5,0(1)$ & $216(7)$ & $4,880(1)$ \\
23 & $58,67(7)$ & $9,85(8)$ & $4,6(2)$ & $5,8(6)$ & $0,31(2)$ & $6,5(2)$ & $231(12)$ & $5,035(3)$ \\
23,5 & $58,24(5)$ & $9,69(6)$ & $4,6(1)$ & $4,9(3)$ & $0,31(2)$ & $5,7(1)$ & $205(6)$ & $5,131(2)$ \\
24 & $58,00(6)$ & $9,65(8)$ & $4,5(2)$ & $4,5(3)$ & $0,28(2)$ & $5,7(1)$ & $189(8)$ & $5,209(3)$ \\
24,5 & $57,74(6)$ & $9,54(7)$ & $4,5(2)$ & $4,5(3)$ & $0,28(2)$ & $5,9(1)$ & $203(8)$ & $5,246(2)$ \\
25 & $57,54(8)$ & $9,47(9)$ & $4,5(2)$ & $4,4(3)$ & $0,29(2)$ & $6,1(2)$ & $201(10)$ & $5,308(3)$ \\
26 & $57,13(9)$ & $9,34(9)$ & $4,6(3)$ & $4,5(5)$ & $0,32(3)$ & $6,9(3)$ & $229(14)$ & $5,397(4)$ \\
28 & $56,73(6)$ & $9,14(8)$ & $4,2(1)$ & $3,7(2)$ & $0,27(2)$ & $6,5(2)$ & $193(8)$ & $5,619(4)$ \\
31 & $55,91(8)$ & $8,77(9)$ & $4,3(2)$ & $3,1(2)$ & $0,28(3)$ & $7,1(2)$ & $186(8)$ & $5,936(5)$ \\
\hline
\end{tabular}

DMPC/Cholesterin 70:30

\begin{tabular}{|c|c|c|c|c|c|c|c|c|}
\hline $\mathrm{T}\left[{ }^{\circ} C\right]$ & $\delta \epsilon_{3}$ & $\tau_{3}[\mathrm{~ns}]$ & $\delta \epsilon_{2}$ & $\tau_{2}[\mathrm{~ns}]$ & $\alpha$ & $\delta \epsilon_{1}$ & $\tau_{1}[\mathrm{~ns}]$ & $\sigma \frac{m S}{m}$ \\
\hline 18 & $60,91(6)$ & $10,71(8)$ & $4,3(2)$ & $7,2(5)$ & $0,29(2)$ & $5,5(3)$ & $321(22)$ & $3,698(3)$ \\
21 & $59,86(7)$ & $10,22(9)$ & $3,9(2)$ & $5,0(4)$ & $0,26(3)$ & $3,4(1)$ & $191(13)$ & $3,951(2)$ \\
23 & $58,99(5)$ & $9,80(7)$ & $3,7(1)$ & $4,3(3)$ & $0,22(2)$ & $3,8(1)$ & $178(10)$ & $4,098(2)$ \\
23,5 & $58,47(8)$ & $9,74(9)$ & $4,0(2)$ & $4,4(5)$ & $0,27(3)$ & $4,8(3)$ & $243(25)$ & $4,114(4)$ \\
24 & $58,20(5)$ & $9,62(7)$ & $3,7(1)$ & $4,2(2)$ & $0,22(2)$ & $4,5(1)$ & $202(9)$ & $4,168(2)$ \\
24,5 & $57,67(4)$ & $9,59(6)$ & $4,1(1)$ & $4,2(2)$ & $0,28(2)$ & $4,7(1)$ & $241(8)$ & $4,199(2)$ \\
25 & $57,25(5)$ & $9,46(7)$ & $3,6(1)$ & $3,9(2)$ & $0,23(2)$ & $4,7(1)$ & $203(9)$ & $4,217(2)$ \\
26 & $56,45(6)$ & $9,36(9)$ & $3,7(2)$ & $3,9(3)$ & $0,24(3)$ & $5,2(2)$ & $239(13)$ & $4,267(2)$ \\
28 & $55,75(5)$ & $9,03(7)$ & $3,6(1)$ & $3,5(2)$ & $0,23(2)$ & $5,1(1)$ & $218(8)$ & $4,425(2)$ \\
31 & $54,66(6)$ & $8,68(9)$ & $3,7(1)$ & $2,8(2)$ & $0,25(3)$ & $5,5(1)$ & $216(9)$ & $4,659(2)$ \\
\hline
\end{tabular}

Tabelle 4.2: Parameter aus der Anpassung der Gleichung (4.37) an die Spektren der Suspensionen DMPC, DMPC/Cholesterin 85:15 und DMPC/Cholesterin 70:30 
DMPC/DPPC 50:50

\begin{tabular}{|c|c|c|c|c|c|c|c|}
\hline $\mathrm{T}\left[{ }^{\circ} C\right]$ & $\delta \epsilon_{1}$ & $\tau_{1}[\mathrm{~ns}]$ & $\alpha$ & $\delta \epsilon_{2}$ & $\tau_{2}[\mathrm{~ns}]$ & $\alpha$ & $\sigma \frac{m S}{m}$ \\
\hline 25 & 3 & $65(60)$ & 0,3 & $10,7(7)$ & $57(57)$ & $0,33(7)$ & $16,424(5)$ \\
28 & 3 & $61(60)$ & 0,3 & $12(2)$ & $69(69)$ & $0,38(8)$ & $17,28(1)$ \\
30 & 3 & $25(22)$ & 0,3 & $12,7(6)$ & $82(80)$ & $0,38(8)$ & $17,75(1)$ \\
31 & 3 & $6,9(15)$ & 0,3 & $11,9(4)$ & $84(6)$ & $0,27(3)$ & $17,949(5)$ \\
32 & $2(1)$ & $2,4(10)$ & $0,1(3)$ & $13(2)$ & $64(8)$ & $0,27(8)$ & $17,925(9)$ \\
33 & $3,3(8)$ & $3,8(6)$ & $0,3(2)$ & $12(1)$ & $68(5)$ & $0,23(5)$ & $17,952(6)$ \\
34 & $3,0(6)$ & $3,0(5)$ & $0,3(2)$ & $12,5(8)$ & $62(3)$ & $0,24(4)$ & $17,731(4)$ \\
35 & $3,6(6)$ & $3,1(5)$ & $0,2(1)$ & $9,8(8)$ & $60(3)$ & $0,09(4)$ & $17,8833(5)$ \\
37 & $3,4(7)$ & $2,1(3)$ & $0,2(2)$ & $11,9(9)$ & $60(5)$ & $0,18(6)$ & $18,378(9)$ \\
40 & $3(1)$ & $1,7(6)$ & $0,2(2)$ & $14(2)$ & $60(11)$ & $0,2(1)$ & $19,28(2)$ \\
\hline
\end{tabular}

DMPC/DMG 75:25

\begin{tabular}{|c|c|c|c|c|c|c|c|c|}
\hline $\mathrm{T}\left[{ }^{\circ} C\right]$ & $\delta \epsilon_{1 a}$ & $\tau_{1 a}[\mathrm{~ns}]$ & $\delta \epsilon_{1 b}$ & $\tau_{1 b}[\mathrm{~ns}]$ & $\delta \epsilon_{2}$ & $\tau_{2}[\mathrm{~ns}]$ & $\alpha$ & $\sigma \frac{m S}{m}$ \\
\hline 20 & $12(5)$ & $188(20)$ & $52(5)$ & $560(60)$ & $5(5)$ & $27(19)$ & $0,4(4)$ & $4(2)$ \\
25 & $6(5)$ & $135(20)$ & $54(5)$ & $444(60)$ & $5(5)$ & $23(19)$ & $0,39(3)$ & $4(1)$ \\
30 & $20(3)$ & $201(20)$ & $57(7)$ & $624(60)$ & $5(5)$ & $19(19)$ & $0,38(3)$ & $4,31(4)$ \\
35 & $16(3)$ & $152(20)$ & $61(7)$ & $499(60)$ & $4(3)$ & $9(8)$ & $0,32(3)$ & $4,6(3)$ \\
40 & $22(2)$ & $166(9)$ & $79(4)$ & $614(55)$ & $4(5)$ & $6(5)$ & $0,3(1)$ & $4,88(3)$ \\
45 & $19(2)$ & $141(9)$ & $95(4)$ & $535(36)$ & $3,3(3)$ & $2,9(3)$ & $0,23(9)$ & $5,27(3)$ \\
50 & $25(3)$ & $146(11)$ & $110(7)$ & $598(57)$ & $3,6(4)$ & $2,3(2)$ & $0,3(1)$ & $5,54(5)$ \\
55 & $23(4)$ & $130(11)$ & $109(7)$ & $533(59)$ & $3,5(4)$ & $1,5(2)$ & $0,2(1)$ & $5,93(6)$ \\
60 & $23(4)$ & $120(10)$ & $113(11)$ & $520(69)$ & $3,1(4)$ & $1,1(2)$ & $0,2(1)$ & $6,24(7)$ \\
\hline
\end{tabular}

DMPC/DPPC 50:50

DMPC/DMG 75:25

\begin{tabular}{|c|c|c||c|c|c|}
\hline $\mathrm{T}\left[{ }^{\circ} C\right]$ & $\delta \epsilon_{3}$ & $\tau_{3}[\mathrm{~ns}]$ & $\mathrm{T}\left[{ }^{\circ} C\right]$ & $\delta \epsilon_{3}$ & $\tau_{3}[\mathrm{~ns}]$ \\
\hline 25 & $60,62(5)$ & $9,6(1)$ & 20 & $56,3(1)$ & $9,61(9)$ \\
28 & $59,3(1)$ & $9,2(3)$ & 25 & $55,44(1)$ & $9,1(1)$ \\
30 & $57,9(1)$ & $8,9(1)$ & 30 & $54,23(6)$ & $8,2(2)$ \\
31 & $57,0(1)$ & $8,8(2)$ & 35 & $53,11(6)$ & $7,8(2)$ \\
32 & $55,7(2)$ & $8,7(2)$ & 40 & $51,7(6)$ & $7,0(2)$ \\
33 & $54,4(2)$ & $8,5(3)$ & 45 & $50,8(2)$ & $6,9(3)$ \\
34 & $52,8(1)$ & $8,2(9)$ & 50 & $50,1(3)$ & $6,6(4)$ \\
35 & $52,1(1)$ & $8,4(3)$ & 55 & $48,9(3)$ & $6,3(5)$ \\
37 & $50,8(2)$ & $8,1(5)$ & 60 & $47,9(3)$ & $6,0(6)$ \\
40 & $49,8(6)$ & $8(1)$ & & & \\
\hline
\end{tabular}

Tabelle 4.3: Parameter aus der Anpassung der Gleichung (4.38) bzw. (4.39) an die Spektren der Suspensionen DMPC/DPPC 50:50 und DMPC/DMG 75:25. Die kursiv gedruckten Werte wurden bei der Anpassung festgehalten. 


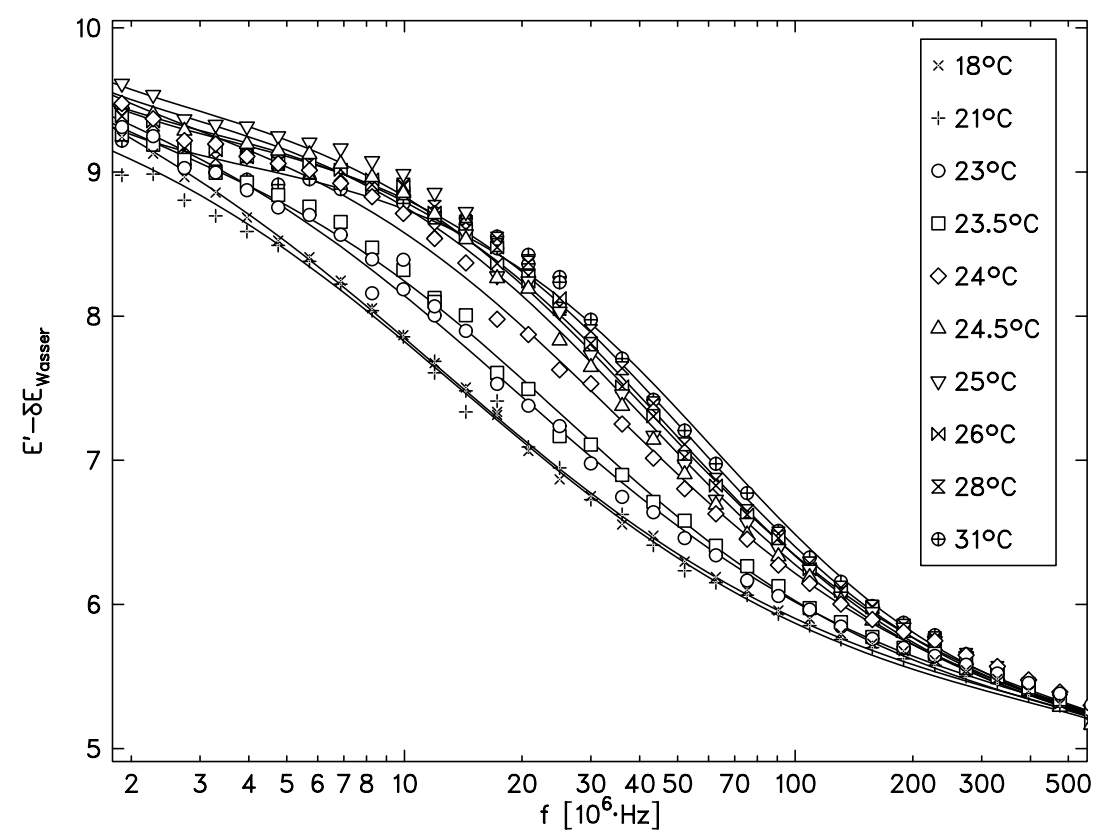

Abb. 4.11: Realteil des dielektrischen Spektrums aller DMPC Suspensionen zur Verdeutlichung der Temperaturabhängigkeit der Relaxationsstufe jeweils mit angepaßter Spektralfunktion Gleichung (4.37)

\subsection{Parameter der Anpassung}

\subsubsection{Die elektrische Leitfähigkeit}

Die elektrische Leitfähigkeit der Suspensionen $\sigma(0)$ wird mit Hilfe der Anpaßrechnungen der jeweiligen Spektralfunktion (4.37) (4.38) (4.39) an die gemessenen Spektren gewonnen. Da das als Lösungsmittel dienende Wasser eine Leitfähigkeit von ca. $\sigma(0)=0,0004 \frac{S}{m}$ besitzt, kann aufgrund der gemessenen Leitfähigkeiten der Suspensionen, die in etwa eine Größenordnung höher liegen, darauf geschlossen werden, daß die resultierenden Leitfähigkeiten auf unerwünschte Verunreinigungen zurückzuführen sind. Diese Verunreinigungen können, wie schon erwähnt, durch direkte Unreinheiten des Trockenpulvers oder durch die Extrusionsprozedur bedingt sein. Abb. 4.12 zeigt den Verlauf der Leitfähigkeiten der Suspensionen DMPC, DMPC/Cholesterin 85:15, DMPC/Cholesterin 70:30 und DMPC/DMG 75:25. Bei allen Suspensionen ist eindeutig ein linearer Verlauf mit der Temperatur auszumachen und spezifische Effekte, die eventuell auf die Membranumwandlung zurückzuführen wären, sind nicht auszumachen. Dagegen zeigt die Suspension DMPC/DPPC 50:50 in Abb. 4.13, deren Leitfähigkeit etwa eine Größenordnung über der der anderen Suspensionen liegt, eindeutig einen Verlauf, bei dem die Hauptumwandlung $T_{m}=33{ }^{\circ} \mathrm{C}$ mit eine Rolle spielt. Dieser charakteristische Verlauf findet sich ebenfalls in [131], wo Suspensionen von DMPC/DMPG untersucht werden, also ganz bewußt Lipide mit Gegenionen in die Membran eingebracht werden. Zusammenfassend läßt sich folglich sagen, daß der beobachtete Effekt bei der DMPC/DPPC 50:50 Lösung auf Verunreinigungen der Trockenpulvers durch Lipide mit Gegenionen (also hier DPPG) zurückzuführen ist. 


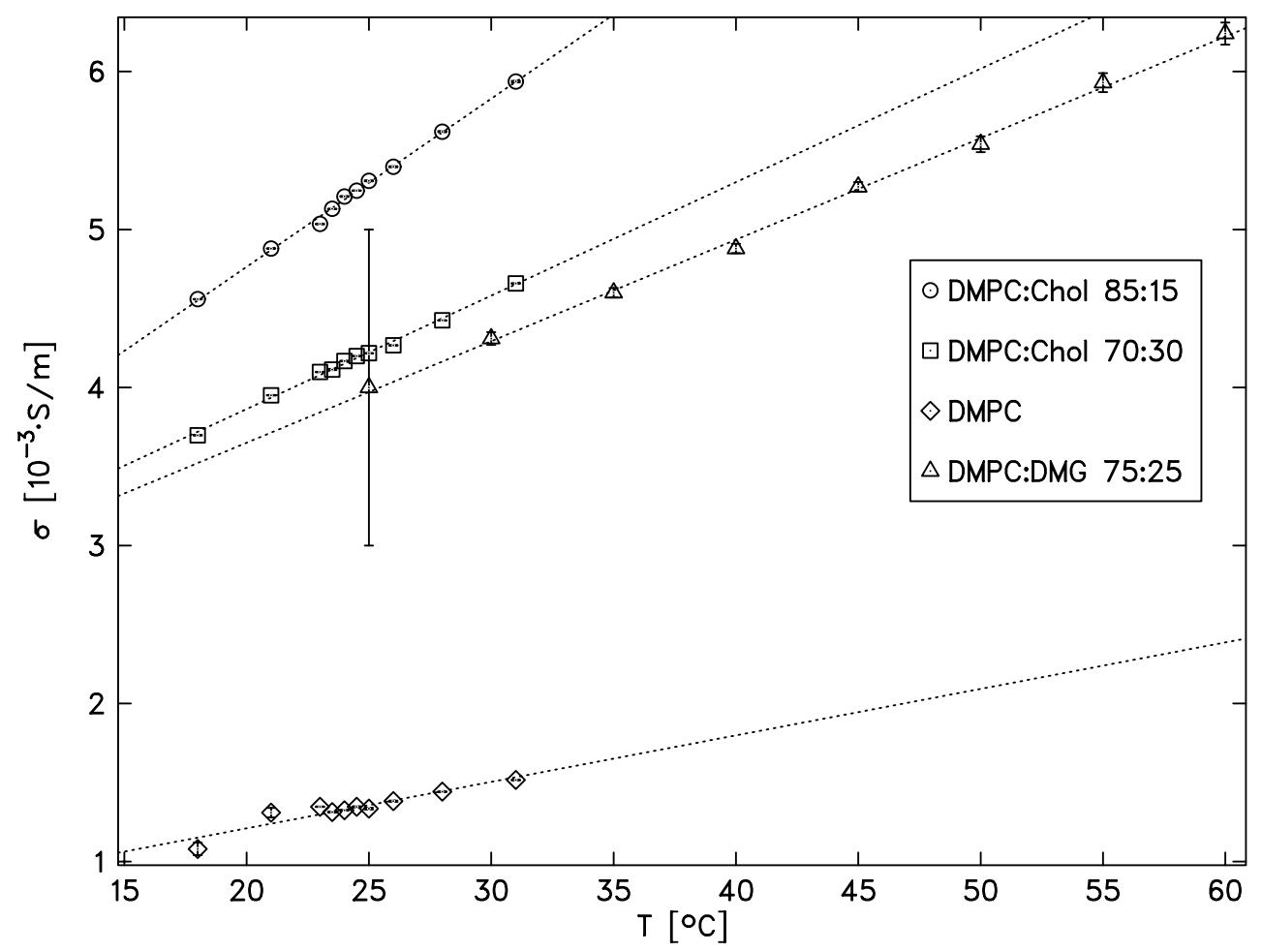

Abb. 4.12: Verlauf der Leitfähigkeiten der Suspensionen DMPC, DMPC/Cholesterin 85:15, DMPC/Cholesterin 70:30 und DMPC/DMG 75:25 über der Temperatur

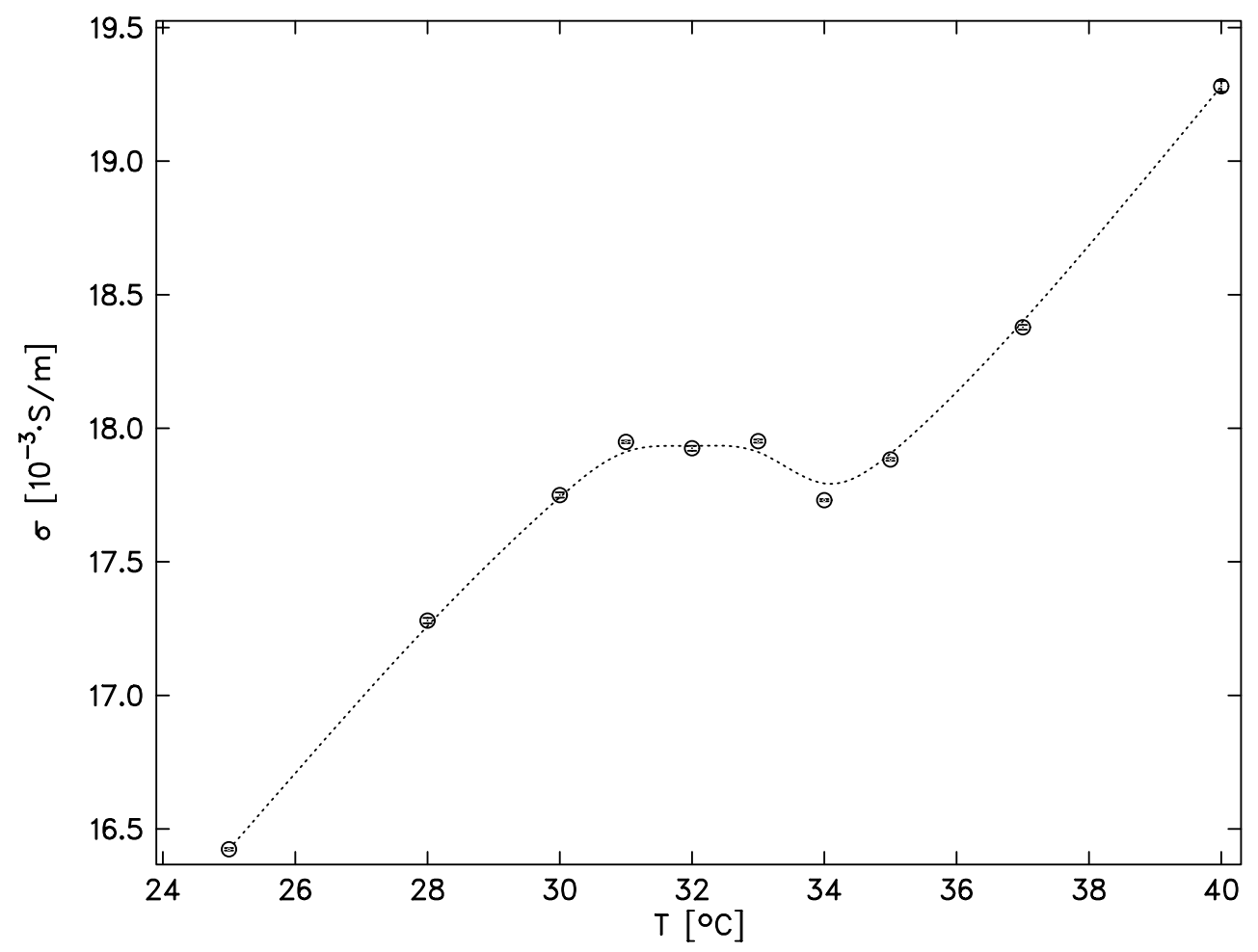

Abb. 4.13: Verlauf der Leitfähigkeiten der Suspensionen DMPC/DPPC 50:50 über der Temperatur 


\subsubsection{Der tieffrequente Relaxationsterm}

Aufgrund von Ionen, die sich zusätzlich zu den Lipiden in der Lösung befinden, ergibt sich durch die begrenzte Bewegung der Ionen in lateraler Richtung auf der Membran der Vesikel ein tieffrequenter Relaxationsterm. Er hängt u.a. von der Ionenkonzentration, der Dicke der Debye-Hückel-Schicht, der Größe der Vesikel und dem thermodynamischen Zustand der Membran ab. In [45] entwickelt C. Grosse eine entsprechende theoretische Beschreibung und kommt zu dem Ergebnis, daß eine symmetrische Relaxationszeitverteilung diesen Effekt sehr gut beschreibt. Da jedoch in den hier durchgeführten Messungen dieser Relaxationsbeitrag nur am unteren Ende des gemessenen Frequenzbereiches auftritt, kann zumeist auf die Anpassung eines Cole-Cole-Terms verzichtet werden und es wird stattdessen ein Debye-Term verwendet. Bei der DMPC/DPPC 50:50 Suspension ist der Gegenionendiffusionseffekt aufgrund der hohen Leitfähigkeit relativ gut ausgeprägt, und es kann ein Cole-ColeTerm zur Anpassung verwendet werden. Dieses deutet entweder auf eine zu den anderen Suspensionen verschiedene Beweglichkeit der Ionen oder auf eine andere Größenverteilung der Vesikel hin. Eine Sonderrolle spielt die DMPC/DMG 75:25 Messung, die trotz vergleichsweise niedriger Leitfähigkeit einen starken Anstieg des Realteils der Dielektrizitätszahl im tieffrequenten Bereich aufweist. Aufgrund des qualitativen Verlaufs der Parameter aus Tabelle 4.2 und 4.3 im Vergleich zu den Daten in [131] wird nun jeweils der tieffrequente Relaxationsbeitrag dieser Ionenbewegung um die Vesikel herum zugeordnet. Da jedoch die Aufmerksamkeit in dieser Arbeit bei der Umorientierung der Kopfgruppen liegt, soll auf den tieffrequenten Relaxationsbeitrag hier nicht detailliert eingegangen werden.

\subsubsection{Der hochfrequente Relaxationsterm}

In den dielektrischen Spektren in den Abb. 4.4 bis 4.10 läßt sich oberhalb von ca. 1 GHz der tieffrequente Ausläufer der Wasserrelaxation ausmachen [74]. Aufgrund der Tatsache, daß sich die Wasserrelaxation im Imaginärteil deutlicher zeigt (Abb. 4.5), kann mittels der Anpassung eines Debye-Terms an diesen hochfrequenten Teil der Spektren grob die Amplitude und die Relaxationszeit der Wasserrelaxation unter dem Einfluß der Phospholipidvesikel bestimmt werden. Die Werte sind in Tabelle 4.2 und 4.3 angegeben. Um jedoch verläßliche Aussagen über die molekularen Vorgänge machen zu können, müßte die Wasserrelaxationsstufe bis ca. $80 \mathrm{GHz}$ voll ausgemessen werden, was im Rahmen dieser Arbeit nicht möglich war.

\subsubsection{Der mittelfrequente Relaxationsterm}

In allen oben dargestellten dielektrischen Spektren läßt sich etwa in der Mitte des gemessenen Frequenzbereiches bei ca. 50 bis $100 \mathrm{MHz}$ ein weiterer Relaxationsprozeß ausmachen, der der Umlagerung der polaren Kopfgruppen der Lipide zugeordnet wird [120] [71] [79] [46] [57]. Abb. 4.14 und 4.15 zeigen den Verlauf der Höhe dieser Relaxationsstufe über der Temperatur, während in Abb. 4.16, 4.17 und 4.18 der Verlauf der jeweiligen Relaxationszeit dargestellt ist. Der Verlauf der Relaxationsstufe zeigt keine besonderen Merkmale im Hinblick auf die charakteristische Umwandlungstemperatur der Lipidmembranen. Jedoch ist diese bei der Relaxationszeit eindeutig auszumachen, welche sich im Verlauf der Temperatur um eine bis anderthalb 


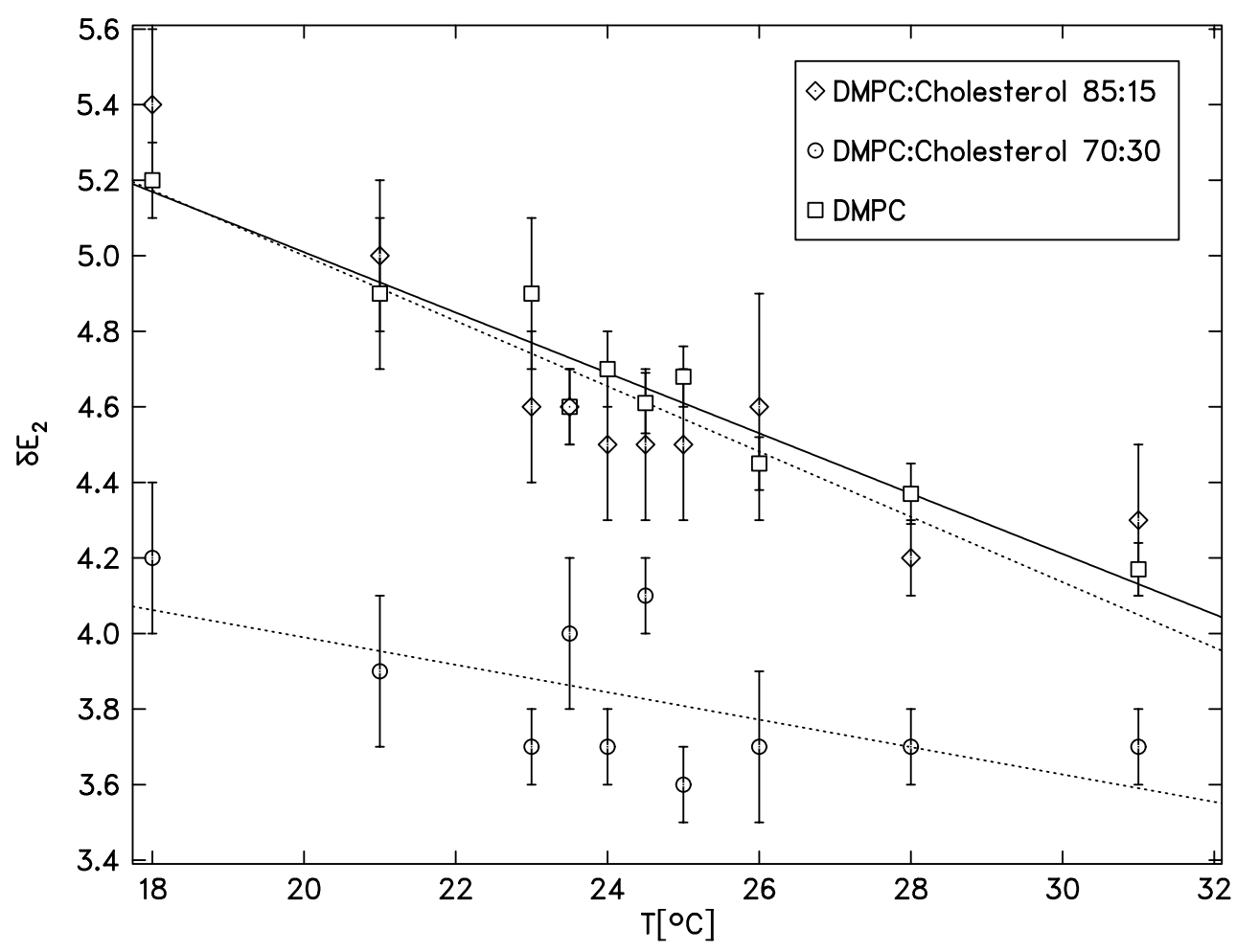

Abb. 4.14: Relaxationsstufe $\delta \epsilon_{2}$ der Kopfgruppenumlagerung der gemessenen Suspensionen DMPC, DMPC/Cholesterin 85:15 und DMPC/Cholesterin 70:30 als Funktion der Temperatur

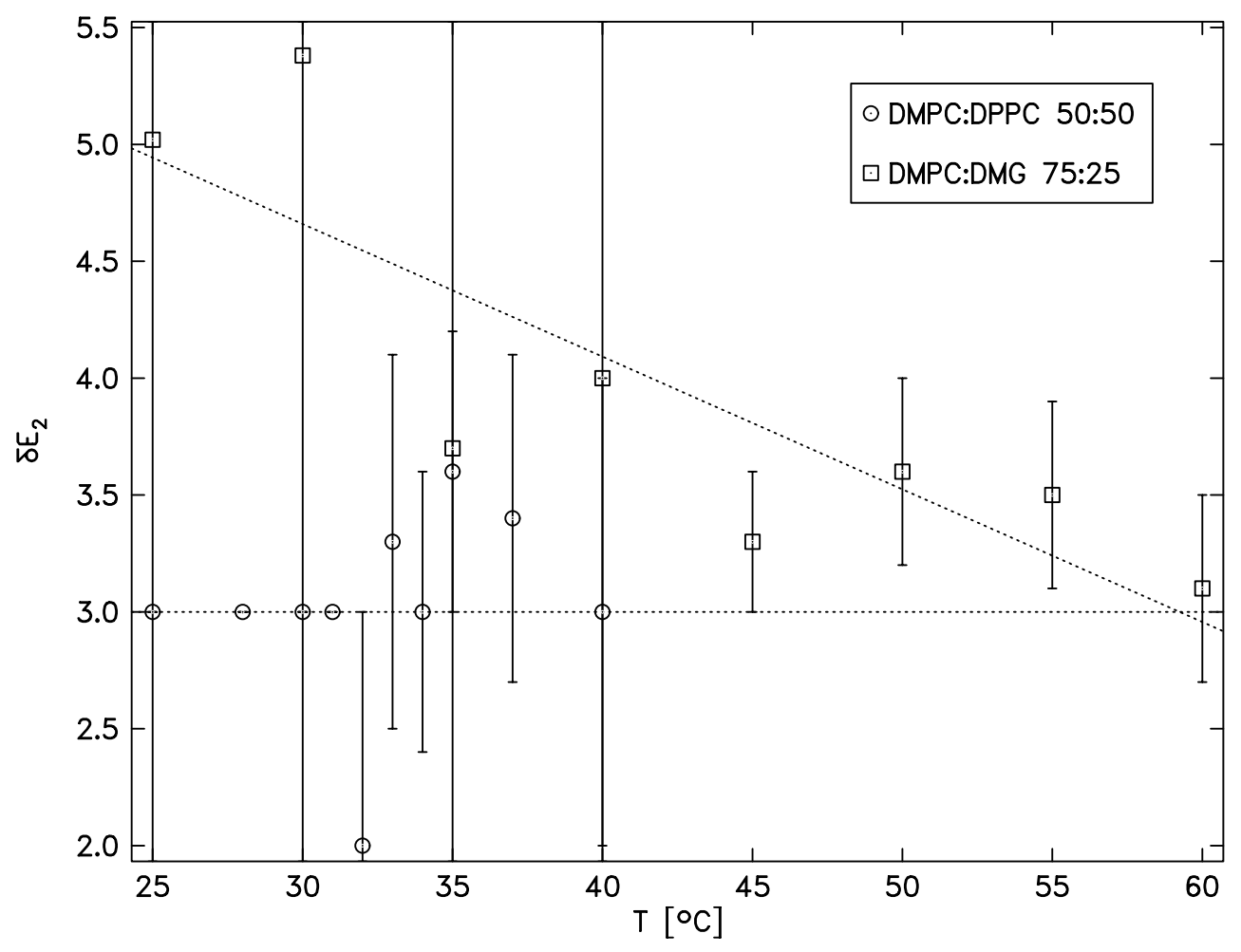

Abb. 4.15: Relaxationsstufe $\delta \epsilon_{2}$ der Kopfgruppenumlagerung der gemessenen Suspensionen DMPC/DPPC 50:50 und DMPC/DMG 75:25 als Funktion der Temperatur 


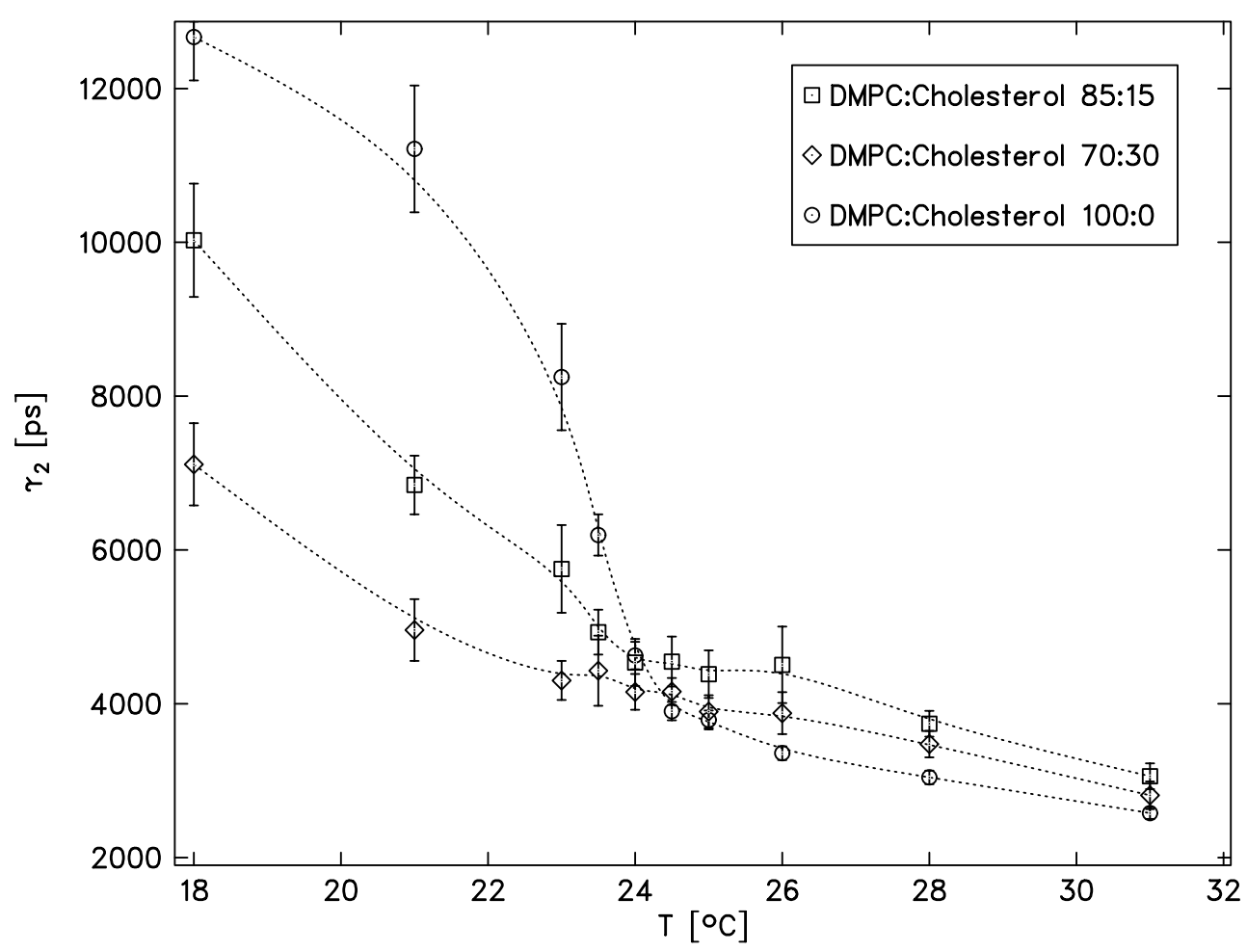

Abb. 4.16: Relaxationszeit $\tau_{2}$ der Kopfgruppenumlagerung der gemessenen Suspensionen DMPC, DMPC/Cholesterin und DMPC/Cholesterin 70:30 als Funktion der Temperatur

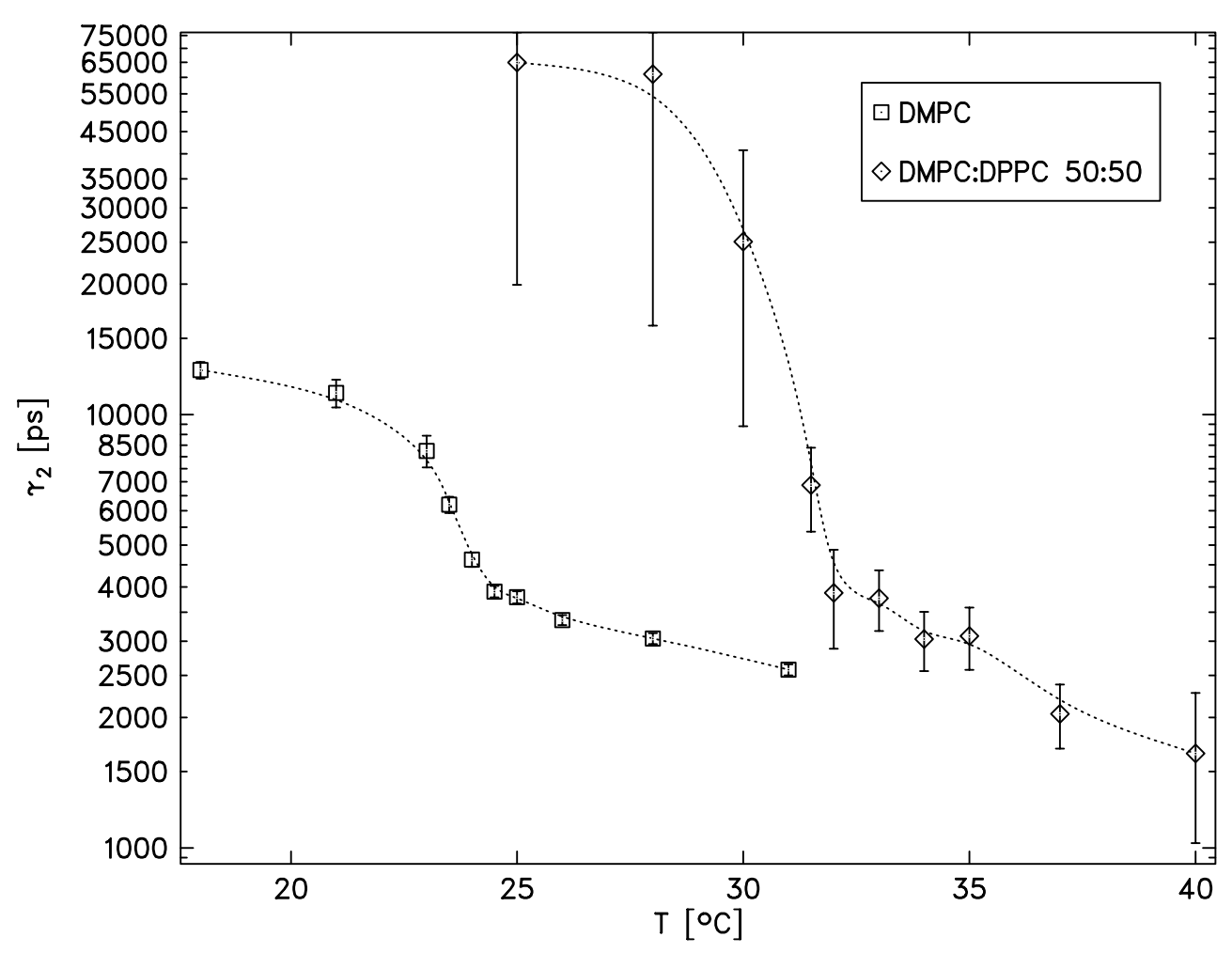

Abb. 4.17: Relaxationszeit $\tau_{2}$ der Kopfgruppenumlagerung der gemessenen Suspensionen DMPC, DMPC/DPPC 50:50 als Funktion der Temperatur 


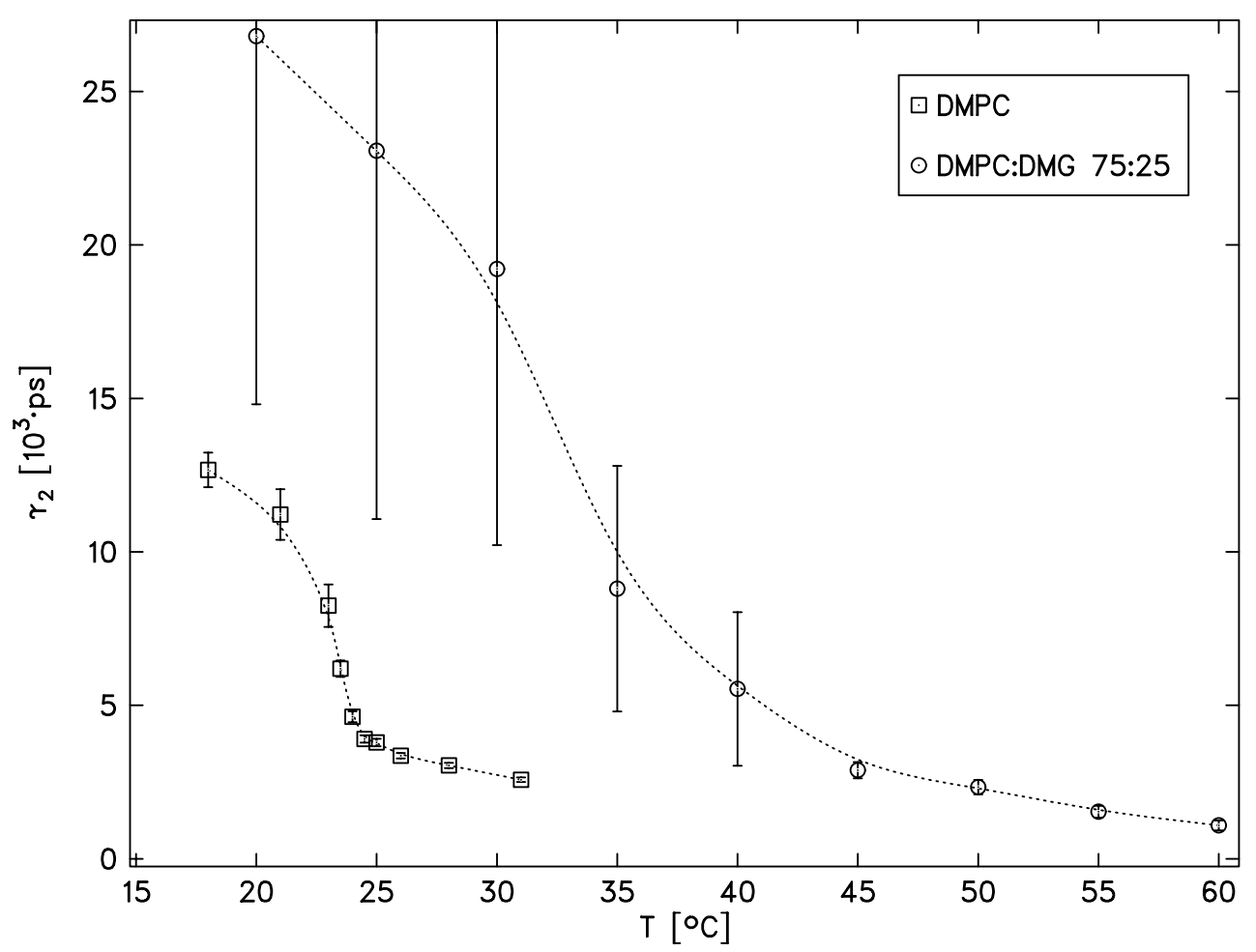

Abb. 4.18: Relaxationszeit $\tau_{2}$ der Kopfgruppenumlagerung der gemessenen Suspensionen DMPC, DMPC/DMG 75:25 als Funktion der Temperatur

Größenordnungen ändert. Unterhalb der Hauptumwandlung der Membran verläuft die Relaxation der Kopfgruppen deutlich langsamer als oberhalb, und dies ist bei allen fünf gemessenen Suspensionen sehr ausgeprägt sichtbar. Da jedoch die Höhe der Relaxationsstufe fast keine Änderung bei steigender Temperatur erfährt, läßt das den Schluß zu, daß grundsätzlich alle möglichen Kopfgruppen an der Umlagerung bei jeder Temperatur beteiligt sind. Verschiedene Suspensionen unterscheiden sich aber darin, wieviel Zeit diese Umlagerung in Anspruch nimmt.

\subsubsection{Besonderheiten im tieffrequente Relaxationsterm der DMPC/DMG 75:25 Suspension}

Bemerkenswert ist die Höhe des tieffrequenten Relaxationsterms $\delta \epsilon_{1}=\delta \epsilon_{1 a}+\delta \epsilon_{1 b}$ (Abb. 4.9, Abb. 4.10 und Tabelle 4.3) bei relativ geringer Leitfähigkeit (Abb. 4.12). Die Leitfähigkeit von ca. $4 \mathrm{mS} / \mathrm{m}$ läßt eigentlich eher auf einen ähnlichen tieffrequenten Relaxationsbeitrag wie bei der reinen DMPC Suspension schließen. Dennoch deutet der Verlauf von $\delta \epsilon_{1 b}$, der mit der Membranumwandlung stark ansteigt, auf einen Ionendiffusionsprozeß. Allerdings sind alle anderen zugehörigen Parameter über der Temperatur quasi konstant. Denkbar ist aber auch eine Ionenpaarbildung innerhalb der Membran, verursacht durch das zusätzliche DMG. Da das DMG eine sehr viel kleinere Kopfgruppe als das DMPC besitzt, fügt es sich in anderer Form in die DMPC-Membran als beispielsweise das DPPC. Möglicherweise bindet das DMG in irgendeiner Form an das Glyceringerüst des DMPC und verursacht so eine merkliche Ladungsverschiebung, die dann im dielektrischen Spektrum sichtbar wäre. Da jedoch die Zeiten der in Betracht kommenden Relaxationsprozesse kei- 
nerlei signifikante Änderungen an den Umwandlungstemperaturen der betrachteten DMPC/DMG Suspension zeigen, muß man von dieser Spekulation Abstand nehmen. Möglicherweise ist eine fehlerbehaftete Anpassung aufgrund des unzureichend ausgemessenen Frequenzbereiches für diese Diskrepanzen verantwortlich. In jedem Fall müßte, um aussagekräftige Daten zu erhalten, der Frequenzbereich der Relaxationen vollständig ausgemessen werden. Zusammenfassend läßt sich festhalten, daß sicherlich einer der beiden Relaxationsprozesse einer begrenzten Bewegung von Ionen zuzuordnen ist. Möglicherweise bilden sich größere Cluster von Vesikeln in der Suspension und folglich sind die Diffusionswege der Ionen auf der Membranoberfläche größer, was schließlich in einer höheren Polarisation und Relaxationszeit resultiert.

\subsection{Die Theorie von R. Pottel}

Um von den gemessenen Spektren Rückschlüsse auf molekulare Größen und Ursachen ziehen zu können, muß man mittels entsprechend geeigneter Modelle die spezielle Form der Aggregate berücksichtigen. Pottel et al. stellt in [120] ein passendes Modell multilammelarer Vesikel vor [57]. Es soll nun zunächst die für unilammelare Vesikel vereinfachte Theorie vorgestellt und anschließend durch einen zusätzlichen Relaxationsbeitrag entsprechend erweitert werden.

Die Vesikel werden mit Hilfe einer kugelschalenförmigen Membran gebildet, die aus den Phospholipiden zusammengesetzt ist. Sowohl innerhalb, als auch außerhalb der Schale befindet sich Wasser. Der Durchmesser der hier betrachteten Vesikel liegt in der Größenordnung von ca. $100 \mathrm{~nm}$. Die zu den Phospholipidkopfgruppen gehörenden Trimethylammoniumgruppen (TMA-Gruppen) können nun aufgrund ihrer Beweglichkeit mit dem von außen anliegenden elektrischen Wechselfeld interagieren. Die positive Ladung $e_{0}$ der TMA-Gruppen, wird in dem Modell durch eine mittlere Flächenladungsdichte $\bar{n} \cdot e_{0}$ ersetzt. Die TMA-Ionen sollten mit der Beweglichkeit $u_{I}$ eine Diffusionsbewegung relativ zu der negativ geladenen Phosphatidylgruppe auf einer Kreisbahn mit dem Radius $\xi$ auf der Membranoberfläche ausführen können und so die Orientierungspolarisierbarkeit der Oberfläche erzeugen. Dabei sei die Bewegung einer mittleren Anzahl $g_{I}$ vollständig miteinander korreliert. Nun kann der Wasserkern und die polarisierbare Innenfläche der Membran zu einer Kugel aus einen homogenen Ersatzdielektrikum mit folgender Dielektrizitätszahl zusammengefaßt werden:

$$
\begin{gathered}
\epsilon_{i}=\epsilon_{w}(\omega)+\epsilon_{s}(\omega) \\
\operatorname{mit} \epsilon_{s}(\omega)=g_{I} \bar{n} \cdot \frac{4 \pi}{r_{w}} \cdot \frac{\left(e_{0} \xi\right)^{2} / k T}{1+i \omega \tau_{s}}=\frac{\Delta \epsilon_{s}}{1+i \omega \tau_{s}} \\
\operatorname{mit} \tau_{s}=\frac{\xi^{2}}{u_{I} k T} .
\end{gathered}
$$

Dabei ist $\epsilon_{w}(\omega)$ die Dielektrizitätszahl des Wasserkerns, k die Bolzmannkonstante, $\mathrm{T}$ die absolute Temperatur und $r_{w}$ der Innenradius der Vesikel.

Der innere Bereich der Membran wird maßgeblich durch die Kohlenwasserstoffketten dominiert. Die Einbeziehung dieses Teils und der äußeren polarisierbaren Oberfläche 
mit dem Radius $r_{a}$ ergibt für die Dielektrizitätszahl des homogenen Dielektrikums der dielektrisch äquivalenten Ersatzkugel:

$$
\epsilon_{v}=\epsilon_{c} \cdot \frac{\left(2 \epsilon_{c}+\epsilon_{i}\right) r_{a}^{3}-2\left(\epsilon_{c}-\epsilon_{i}\right) r_{w}^{3}}{\left(2 \epsilon_{c}+\epsilon_{i}\right) r_{a}^{3}+\left(\epsilon_{c}-\epsilon_{i}\right) r_{w}^{3}}+\frac{r_{w}}{r_{a}} \cdot \epsilon_{s}
$$

$\epsilon_{c}$ ist dabei die Dielektrizitätszahl des Kohlenwasserstoffbereiches innerhalb der Membran, die, entsprechend der Dielektrizitätszahl flüssiger Alkane, $\epsilon_{c}=2$ gesetzt wird.

Nun muß noch die Tatsache berücksichtigt werden, daß sie gemessene Flüssigkeit eine Mischung aus Wasser und kugelförmigen Vesikeln ist. Durch Verwendung der Maxwell-Wagner-Formel für die Dielektrizitätszahl eines Mischungsdielektrikums aus sphärischen, homogenen Teilchen mit der Dielektrizitätszahl $\epsilon_{v}$ in einem Kontinuum $\epsilon_{w}$ [15], erhält man schließlich die resultierende Dielektrizitätszahl der Modellösung:

$$
\epsilon(\omega)=\epsilon_{w}+3 b_{e} \epsilon_{w} \cdot \frac{\epsilon_{v}-\epsilon_{w}}{2 \epsilon_{w}+\epsilon_{v}+f_{e}\left(\epsilon_{w}-\epsilon_{v}\right)} .
$$

Dabei ist $b_{e}$ der Volumenbruchteil der Aggregate mit der Dielektrizitätszahl $\epsilon_{a}$, welche alle Vesikel zusätzlich dem Hydratwasser beinhaltet.

$$
b_{e}=\frac{V_{z}}{V}=\frac{V_{z}}{V_{l}} \cdot \frac{V_{l}}{V}=\frac{1}{f_{i}} \cdot b
$$

$V, V_{l}$ und $V_{z}$ sind die Volumina der Lösung, des Lipids und der Aggregate. $b$ und $b_{i}$ sind die Volumenbrüche, Lipidvolumen pro Lösungsvolumen bzw. Lipidvolumen pro Aggregatvolumen. $b$ läßt sich recht einfach aus der Wasserkonzentration berechnen:

$$
b=1-\frac{C_{w}}{C_{w 0}} \quad, \quad \text { mit } C_{w 0}=55,34 \mathrm{~mol} / 1 \text { bei } 25^{\circ} \mathrm{C} .
$$

$b_{i}$ berechnet sich mit Hilfe der Volumina der Vesikel bzw. der Kugelschalen bestehend aus der Membran:

$$
b_{i}=\frac{V_{l}}{V_{z}}, \quad V_{z}=\frac{4}{3} \pi r_{a}^{3}, \quad V_{l}=\frac{4}{3} \pi\left(r_{a}^{3}-r_{w}^{3}\right)
$$

\subsubsection{Die Oberflächenpolarisierbarkeit}

In der oben dargestellten theoretischen Berschreibung der Relaxation wird ein Debyeähnlicher Verlauf für die Relaxation der Kopfgruppen angenommen. Die empirischen Anpassungen ergaben jedoch, daß nur eine Relaxationszeitverteilung die Spektren hinreichend beschreiben kann.

Bis jetzt wird vereinfachend angenommen, daß sich die TMA-Gruppen an der Oberfläche der Membran auf einer starren Kreisbahn mit dem Radius $\xi$ um den negativen Ladungsschwerpunkt der Kopfgruppe bewegen können. Es ist jedoch leicht denkbar, daß auch Bewegungsmöglichkeiten senkrecht zur lateralen Ebene der Membran vorhanden sind. Dabei bewegen sich die ionischen TMA-Gruppen etwa parallel zur Membrannormalen. Die polare Kopfgruppe kippt dann unter dem Einfluß des elektrischen Wechselfeldes leicht gegen die laterale Ebene der Membran (siehe Abb. 4.19) und erzeugt so einen zusätzlichen Beitrag zu Oberflächenpolarisierbarkeit. 


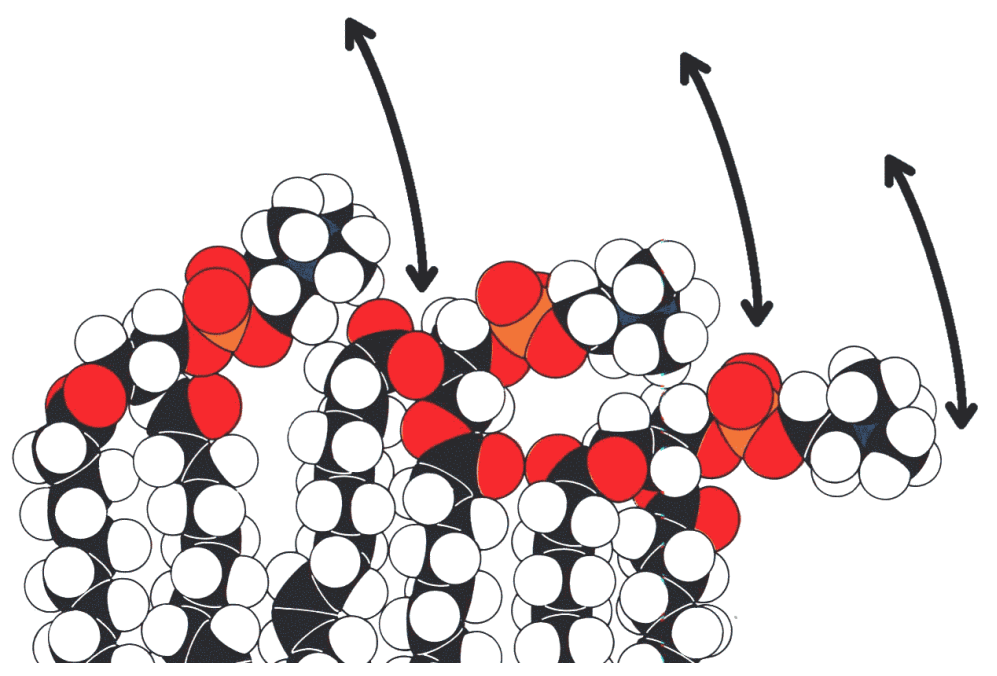

Abb. 4.19: Die Kopfgruppen können gegen die Ebene der Membran kippen.

Für diesen Prozeß ist es sinnvoll, eine entsprechende Beweglichkeit der Kippbewegung einzuführen. Weiter gibt es sicherlich eine maximale Auslenkung der TMAGruppe aus der Membranoberfläche heraus. Die Korrelation der Bewegung der Kopfgruppen der einzelnen Moleküle untereinander wird durch die schon eingeführte Größe $g_{I}$ beschrieben. Letztendlich resultieren diese Annahmen in einer weiteren Relaxationsstufe:

$$
\epsilon_{s 2}(\omega)=\frac{\Delta \epsilon_{s 2}}{1+i \omega \tau_{s 2}}
$$

Somit ergibt sich für die gesamte Dielektrizitätszahl Oberfläche:

$$
\epsilon_{\text {Oberfläche }}=\epsilon_{s}(\omega)+\epsilon_{s 2}(\omega) \text {. }
$$

Es tragen also zwei im Frequenzbereich voneinander getrennte Relaxationsprozesse zur Oberflächenpolarisierbarkeit und damit zum dielektrischen Spektrum bei. Je nach Winkel relativ zu den elektrischen Feldlinien liegt demnach vornehmlich die Kopfgruppenrotation oder die Kippbewegung vor. Da jedoch beide Prozesse im Frequenzbereich sehr dicht beinander liegen, ist es nur in Einzelfällen möglich, zwei Prozesse an das Spektrum anzupassen. Dieses gelingt nur bei der reinen DMPC Suspension und dabei auch nur oberhalb der Umwandlungstemperatur. Unter der Umwandlungstemperatur überlagert der Prozeß der Ionenbewegung um die Vesikel herum zu stark den Prozeß der Kopfgruppenumlagerung, so daß eine Anpassung mit zwei Relaxationsprozessen für die Oberflächenpolarisierbarkeit nicht gelingt. Aus diesen Gründen, wird insgesamt darauf verzichtet, zwei getrennte Prozesse anzupassen, und es wird alternativ ausgehend von Gleichung (4.41) von einer symmetrischen Relaxationszeitverteilung ausgegangen:

$$
\epsilon_{\text {Oberfläche }}(\omega)=g_{I} \bar{n} \cdot \frac{4 \pi}{r_{w}} \cdot \frac{\left(e_{0} \xi\right)^{2} / k T}{1+\left(i \omega \tau_{s}\right)^{1-\alpha}}=\frac{\Delta \epsilon_{s}}{1+\left(i \omega \tau_{s}\right)^{1-\alpha}} .
$$

Dabei nehmen nun die Variablen $u_{I}$ und $\xi$ die Rolle von mittleren Größen an, die die Beweglichkeiten und den Diffusionswegradien zusammenfassen, und sind somit 
nicht in der Lage, Aussagen über die einzelnen Relaxationsprozesse zu machen. Der Vergleich der Anpaßrechnungen an die Spektren der DMPC Suspensionen oberhalb der Umwandlungstemperatur zeigt, daß die aus (4.50) resultierende Spektralfunktion nur geringfügig schlechter an die gemessenen Spektren paßt, als die Spektralfunktion bestehend aus zwei getrennten Deybe-Relaxationen.

Letztendlich gibt es viele unterschiedliche Möglichkeiten für die Existenz der Relaxationszeitverteilung. Es ist z.B. denkbar, daß sich die TMA-Gruppen auf Kreisbahnen mit leicht unterschiedlichem Radius bewegen, was zu unterschiedlichen Relaxationszeiten führt. Diese unterschiedlichen Radien könnten durch verschiedene Abstände zwischen den einzelnen Lipidmolekülen oder durch verschiedene normale Postitionen der Lipidmoleküle verursacht sein. Eine andere Möglichkeit besteht in unterschiedlichen Relaxationszeiten zwischen der Innen- und Außenseite der Vesikelmembran. Sicherlich ist durch den geringeren Platz an der Innenseite der Vesikel die Wechselwirkung zwischen der Lipiden, insbesondere deren Kopfgruppen, leicht verschieden gegenüber den Abständen auf der Außenseite der Membran, so daß unterschiedliche Diffusionswegradien, bzw. Relaxationszeiten resultieren. Jedoch ist eine abschließende Klärung der Ursache der Relaxationszeitverteilung aufgrund der begrenzten Aussagekraft der Messungen und der Natur der Relaxationen nicht möglich.

Für die Anpaßrechnungen wird Gleichung (4.44) mit $\epsilon_{\text {Oberfläche }}$ (4.50) statt $\epsilon_{s}$ (4.41) verwendet. Freie Parameter sind dabei die Höhe der Relaxationsstufe $\Delta \epsilon_{s}$, die Relaxationszeit $\tau_{s}$, der Verteilungsparameter $\alpha$ und der Innenradius der Vesikel $r_{w}$. Vorgegeben wird die Relaxationsstufe des Wassers $\Delta \epsilon_{w}$ nach [73] und die Dicke der Lipidschicht $d_{l}$ [53]. In Tabelle 4.4 sind die entsprechenden Größen aufgeführt.

\begin{tabular}{|c|c|c|c|c|}
\hline Suspension & \multicolumn{2}{|c|}{$d_{l}[\mathrm{~nm}]$} & \multicolumn{2}{c|}{$\Phi_{v}[\mathrm{ml} / \mathrm{g}]$} \\
& $T<T_{m}$ & $T>T_{m}$ & $T<T_{m}$ & $T>T_{m}$ \\
\hline DMPC & 4,35 & 3,47 & 0,93 & 0,97 \\
DMPC/Chol 85:15 & 4,35 & 3,47 & 0,93 & 0,97 \\
DMPC/Chol 70:30 & 4,35 & 3,47 & 0,93 & 0,97 \\
DMPC/DPPC 50:50 & 4,57 & 3,70 & 0,959 & 1,001 \\
DMPC/DMG 75:25 & 4,35 & 3,47 & 0,89 & 0,98 \\
\hline
\end{tabular}

Tabelle 4.4: Vorgegebene Größen bei der Anpaßrechnung; jeweils weit unterhalb bzw. weit oberhalb der Phasenumwandlung

Zur Berechnung der Wasserkonzentration in der Lösung wird das scheinbar spezifische Volumen $\Phi_{v}$ der Lipide verwendet. Für den Werteverlauf über der Temperatur der Parameter aus Tabelle 4.4 wird die sog. "fluid fraction" $f$, die aus einer $C_{p^{-}}$ Messung berechnet wird, herangezogen, so daß sich der Temperaturgang als scheinbar stetig und relativ realistisch berechnen läßt.

$$
a(T)=(1-f) \cdot a_{T<T_{m}}+f \cdot a_{T>T_{m}}
$$

Sofern vorhanden, werden für das scheinbar spezifische Volumen $\Phi_{v}$ Meßdaten der Proben verwendet. Dies ist bei den Suspensionen DMPC, DMPC/DMG und DMPC/DPPC der Fall [65] [148]. Bei den anderen Suspensionen muß der Temperaturverlauf nach Gleichung (4.51) berechnet werden [53]. 


\subsection{Ergebnisse der Anpassung der Theorie}

In den Tabellen 4.5, 4.6 und 4.7 sind die Ergebnisse aus den Anpaßrechnungen der theoretischen Spektralfunktion an die gemessenen Spektren dargestellt. Aufgeführt sind alle freien Parameter. Der Radius $r_{w}$ liegt bei den DMPC und DMPC/DPPC Suspensionen unterhalb von $T_{m}$ in der erwarteten Größenordnung von ca. 75 bis 100 $\AA$. Es ist jedoch deutlich eine Vergrößerung des Radius mit der Temperatur auszumachen, was auf ein quellen der Lipidmembranen und damit einer Veränderung der Geometrie der Vesikel zu einer mehr ellipsoiden Form zurückzuführen ist (siehe auch Kapitel 4.7.1). Jedoch sind die Radien zum Teil doppelt bis dreifach so hoch wie erwartet. Möglicherweise ist die Ursache in den sich ergebenden Schwierigkeiten beim Extrudieren zu suchen. Es könnte, verursacht durch die hohe Konzentration, zu einer Bildung von Vesikeln mit grundsätzlich größeren Ausmaßen gekommen sein, oder es liegen in noch signifikanter Konzentration multilammelare Vesikel in der Lösung vor. Insbesondere ist die ungewöhnliche Größe der Vesikel bei den Suspensionen DMPC/DMG und DMPC/DPPC zu sehen. Auffällig ist hier, daß gerade diese Lösungen besonders schwierig und nur mit hohen Aufwand zu extrudieren waren, was sich u.a. in mehrfachem Reißen des Filters aufgrund der relativ hohen Umwandlungstemperatur und der ungewöhnlich hohen Konzentration äußerte. Dieses stützt die Vermutung, daß die zu großen Radien auf eine unzureichende Extrusion zurückzuführen sind.

Sowohl die Relaxationszeiten, als auch die Relaxationsbeiträge entsprechen ziemlich genau den Werten aus der empirischen Anpassung. Auch der Verteilungsparameter $\alpha$ zeigt ein sehr ähnliches Verhalten zu den Werten aus der empirischen Anpassung. Aus der Relaxationszeit $\tau_{s}$ läßt sich nach Gleichung (4.42) der Quotient $u_{I} / \xi^{2}$ aus der Beweglichkeit $u_{I}$ und dem Quadrat des Radius $\xi$ berechnen. Da der Temperaturverlauf von $\xi$ nicht bekannt ist, soll darauf verzichtet werden, die Beweglichkeit selbst zu errechnen. Da der Radius $\xi$ in dem betrachteten Temperaturbereich voraussichtlich keine gravierende Veränderung widerfährt, läßt sich der Verlauf von $u_{I} / \xi^{2}$ im wesentlichen durch die Beweglichkeit $u_{I}$ beschreiben. Bei T. Heimburg [53] wird die Fläche, die ein Lipidmolekül in der Membran belegt, bei DPPC mit 47,4 $\AA^{2}$ für $25{ }^{\circ} \mathrm{C}$ und mit $62,9 \AA^{2}$ für $50{ }^{\circ} \mathrm{C}$ angegeben. Da diese Fläche im Mittel die Fläche ist, auf der sich die Rotationsbewegung der Kopfgruppen abspielen muß oder zumindest ein grobes Maß für die Änderung von $\xi$ ist, kann gefolgert werden, daß der Diffusionswegradius $\xi$ zwar nicht über der Temperatur konstant sein kann, jedoch seine Änderung auch nicht allein für den Werteverlauf des Quotienten $u_{I} / \xi^{2}$ über der Temperatur in Abb. 4.20 verantwortlich ist. Deutlich ist ein Ansteigen dieses Wertes bei allen Suspensionen noch vor der Hauptumwandlung der Lipide zu sehen. Bei DMPC steigt der Quotient ab ca. $23^{\circ} \mathrm{C}$ um etwa das vierfache an, während er bei der DMPC/DPPC 50:50 Suspension sogar ab ca. $30{ }^{\circ} \mathrm{C}$ um eine Größenordnung ansteigt. Die DMPC/DMG 75:25 Suspension zeigt naturgemäß einen flacheren Anstieg des Quotienten und damit der Beweglichkeit. Es sind aber sogar aus der Steigung der Kurve zwei Umwandlungspunkte bei $35{ }^{\circ} \mathrm{C}$ und $55{ }^{\circ} \mathrm{C}$ auszumachen. Die Beweglichkeit der Kopfgruppen der DMPC/Cholesterin Suspensionen zeigt im Vergleich zur reinen DMPC Lösung keine signifikante Änderung. Alle diese Beobachtungen korrespondieren mit dem Modell eines Flüssigkeitskristalls der Membranen. Dabei sind die Beweglichkeiten der einzelnen Moleküle im "festen" also gel-förmigen Zustand 


\section{DMPC}

\begin{tabular}{|c|c|c|c|c|}
\hline $\mathrm{T}\left[{ }^{\circ} \mathrm{C}\right]$ & $\alpha$ & $\delta \epsilon_{s}$ & $\tau_{s}[\mathrm{ps}]$ & $r_{w}[\mathrm{~nm}]$ \\
\hline 18 & $0,40(1)$ & $13,5(3)$ & $15195(738)$ & $87,7(4)$ \\
21 & $0,36(2)$ & $12,4(4)$ & $12145(970)$ & $85,7(4)$ \\
23 & $0,35(1)$ & $11,4(1)$ & $9033(297)$ & $95,3(5)$ \\
23,5 & $0,30(2)$ & $9,5(2)$ & $6643(284)$ & $105,6(5)$ \\
24 & $0,28(2)$ & $8,2(1)$ & $4918(187)$ & $126,3(7)$ \\
24,5 & $0,25(2)$ & $7,2(1)$ & $4109(128)$ & $141,1(9)$ \\
25 & $0,25(2)$ & $6,68(9)$ & $3973(129)$ & $155(1)$ \\
26 & $0,22(1)$ & $6,13(7)$ & $3502(93)$ & $160(1)$ \\
28 & $0,22(2)$ & $5,78(7)$ & $3139(90)$ & $167(1)$ \\
31 & $0,19(2)$ & $5,31(6)$ & $2670(79)$ & $171(1)$ \\
\hline
\end{tabular}

DMPC/Cholesterin 85:15

\begin{tabular}{|c|c|c|c|c|}
\hline $\mathrm{T}\left[{ }^{\circ} \mathrm{C}\right]$ & $\alpha$ & $\delta \epsilon_{s}$ & $\tau_{s}[\mathrm{ps}]$ & $r_{w}[\mathrm{~nm}]$ \\
\hline 18 & $0,41(2)$ & $10,6(4)$ & $12359(1084)$ & $123,8(7)$ \\
21 & $0,37(2)$ & $9,1(3)$ & $7276(461)$ & $127,0(7)$ \\
23 & $0,31(2)$ & $7,9(3)$ & $6038(426)$ & $130,9(9)$ \\
23,5 & $0,31(2)$ & $7,8(2)$ & $5170(279)$ & $136,7(8)$ \\
24 & $0,28(2)$ & $7,3(2)$ & $4735(315)$ & $139(1)$ \\
24,5 & $0,28(2)$ & $7,1(2)$ & $4744(252)$ & $142,6(9)$ \\
25 & $0,29(2)$ & $7,0(2)$ & $4570(235)$ & $144,8(9)$ \\
26 & $0,32(2)$ & $7,1(2)$ & $4702(230)$ & $150(1)$ \\
28 & $0,27(2)$ & $5,7(1)$ & $3861(202)$ & $165(1)$ \\
31 & $0,28(2)$ & $5,2(1)$ & $3143(200)$ & $182(2)$ \\
\hline
\end{tabular}

DMPC/Cholesterin 70:30

\begin{tabular}{|c|c|c|c|c|}
\hline $\mathrm{T}\left[^{\circ} \mathrm{C}\right]$ & $\alpha$ & $\delta \epsilon_{s}$ & $\tau_{s}[\mathrm{ps}]$ & $r_{w}[\mathrm{~nm}]$ \\
\hline 18 & $0,29(2)$ & $8,6(3)$ & $7585(497)$ & $112,6(7)$ \\
21 & $0,26(1)$ & $7,5(2)$ & $5181(217)$ & $117,9(5)$ \\
23 & $0,22(2)$ & $6,6(2)$ & $4466(254)$ & $124,9(8)$ \\
23,5 & $0,27(2)$ & $6,9(2)$ & $4614(264)$ & $131,8(8)$ \\
24 & $0,22(2)$ & $6,1(2)$ & $4297(239)$ & $134,9(5)$ \\
24,5 & $0,28(2)$ & $6,5(1)$ & $4319(180)$ & $142,8(7)$ \\
25 & $0,23(2)$ & $5,6(2)$ & $4017(218)$ & $149(1)$ \\
26 & $0,24(3)$ & $5,2(2)$ & $3986(281)$ & $163(1)$ \\
28 & $0,23(2)$ & $4,8(1)$ & $3560(177)$ & $174(1)$ \\
31 & $0,25(3)$ & $4,2(1)$ & $2865(185)$ & $198(2)$ \\
\hline
\end{tabular}

Tabelle 4.5: Ergebnisse der Anpassung an die Spektren von DMPC, DMPC/Cholesterin 85:15 und DMPC/Cholesterin 70:30 


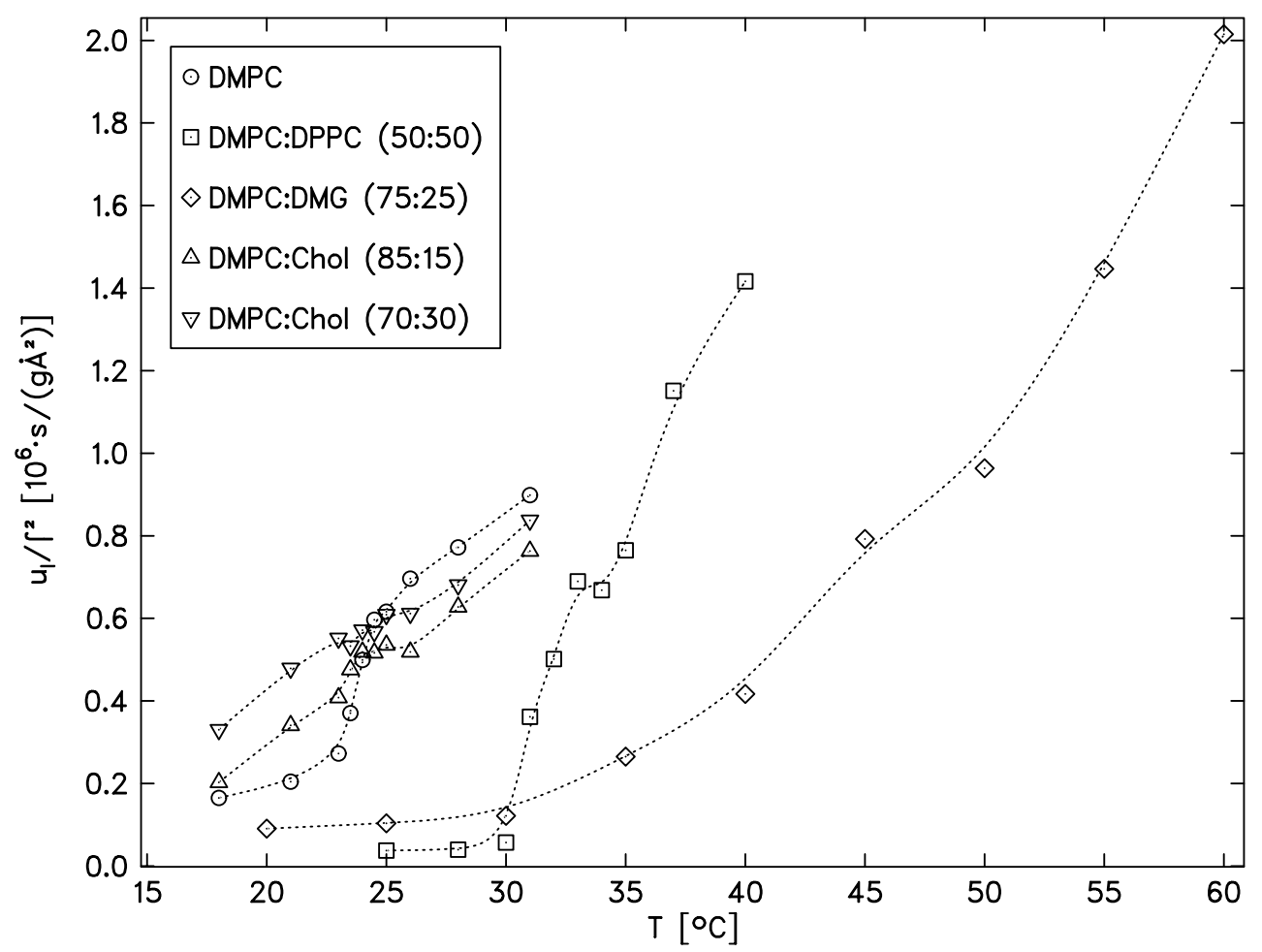

Abb. 4.20: Temperaturverlauf des Quotienten $u / \xi^{2}$ mit der Beweglichkeit $u_{I}$ und dem Radius $\xi$

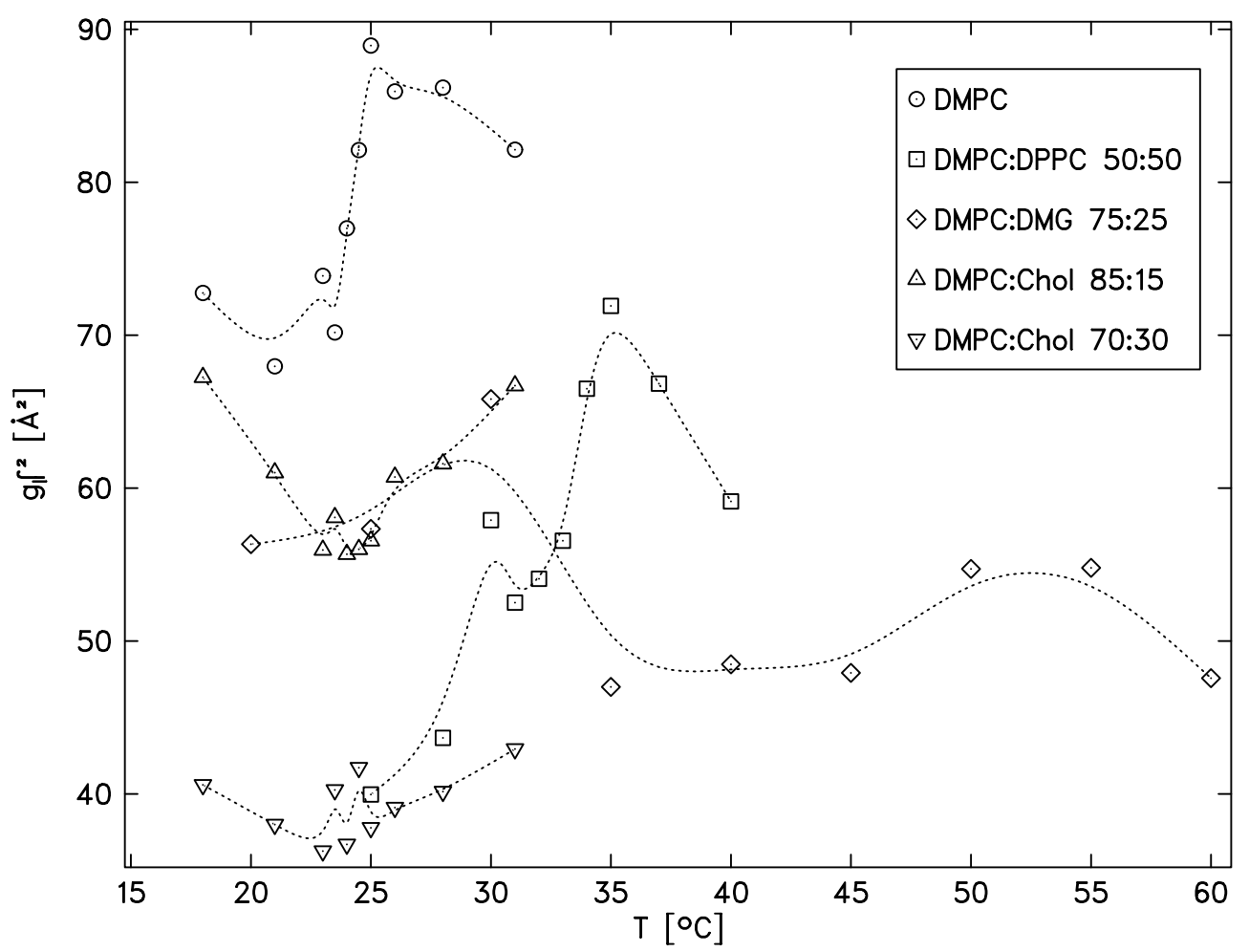

Abb. 4.21: Temperaturverlauf des Produktes $g \cdot \xi^{2}$ mit der Korrelationszahl $g_{I}$ und dem Radius $\xi$ 
DMPC/DMG 75:25

\begin{tabular}{|c|c|c|c|c|}
\hline $\mathrm{T}\left[{ }^{\circ} \mathrm{C}\right]$ & $\alpha$ & $\delta \epsilon_{s}$ & $\tau_{s}[\mathrm{ps}]$ & $r_{w}[\mathrm{~nm}]$ \\
\hline 20 & $0,40(2)$ & $5,9(20)$ & $27443(4000)$ & $213(20)$ \\
25 & $0,39(2)$ & $5,6(20)$ & $23652(4000)$ & $216(20)$ \\
30 & $0,38(2)$ & $5,5(10)$ & $19748(2000)$ & $233(30)$ \\
35 & $0,32(1)$ & $3,5(5)$ & $8931(1000)$ & $247(24)$ \\
40 & $0,29(1)$ & $3,0(2)$ & $5589(298)$ & $271(20)$ \\
45 & $0,24(1)$ & $2,2(2)$ & $2894(298)$ & $330(20)$ \\
50 & $0,26(1)$ & $2,2(2)$ & $2343(270)$ & $350(30)$ \\
55 & $0,24(1)$ & $1,9(1)$ & $1537(222)$ & $394(43)$ \\
60 & $0,16(2)$ & $1,6(1)$ & $1087(189)$ & $400(49)$ \\
\hline
\end{tabular}

Tabelle 4.6: Ergebnisse der Anpassung an die Spektren von DMPC/DMG 75:25

DMPC/DPPC 50:50

\begin{tabular}{|c|c|c|c|c|}
\hline $\mathrm{T}\left[{ }^{\circ} \mathrm{C}\right]$ & $\alpha$ & $\delta \epsilon_{s}$ & $\tau_{s}[\mathrm{ps}]$ & $r_{w}[\mathrm{~nm}]$ \\
\hline 25 & $0,28(20)$ & $6,85(81)$ & $64885(60000)$ & $92(8)$ \\
28 & $0,28(20)$ & $6,74(95)$ & $61159(60000)$ & $99(10)$ \\
30 & $0,43(20)$ & $7,25(90)$ & $42029(21000)$ & $116(11)$ \\
31 & $0,37(20)$ & $5,59(70)$ & $6636(1500)$ & $129(13)$ \\
32 & $0,47(22)$ & $4,15(89)$ & $4769(998)$ & $166(15)$ \\
33 & $0,30(15)$ & $3,36(70)$ & $3455(600)$ & $199(20)$ \\
34 & $0,33(15)$ & $2,63(46)$ & $3554(470)$ & $283(28)$ \\
35 & $0,22(11)$ & $2,43(45)$ & $3095(500)$ & $326(32)$ \\
37 & $0,20(15)$ & $1,72(49)$ & $2043(340)$ & $422(42)$ \\
40 & $0,16(16)$ & $1,28(99)$ & $1645(600)$ & $496(50)$ \\
\hline
\end{tabular}

Tabelle 4.7: Ergebnisse der Anpassung an die Spektren von DMPC/DPPC 50:50

naturgemäß sehr viel geringer als im "flüssigem" also fluidem Zustand.

Aus dem Relaxationsbeitrag $\Delta \epsilon_{s}$ läßt sich nach Gleichung (4.41) das Produkt $g_{I} \cdot \xi^{2}$ aus der Korrelationszahl und dem Quadrat des Radius $\xi$ berechnen. Für die mittlere Flächenanzahldichte $\bar{n}$ der positiv geladenen Gruppen werden dabei die Werte von Phillips et al. [118] $\left(1 / \bar{n}=48 \AA^{2}\right.$ für $T<T_{m}$ und $1 / \bar{n}=70 \AA^{2}$ für $\left.T>T_{m}\right)$ angenommen. Der Temperaturverlauf dieses Parameters wird ebenfalls mittels der "fluid fraction" berechnet. In Abb. 4.21 ist der Verlauf des so ermittelten Produktes $g_{I} \cdot \xi^{2}$ über der Temperatur dargestellt. Auch hier gilt die Annahme, daß sich der Diffusionswegradius $\xi$ wahrscheinlich nicht wesentlich über der Temperatur variiert, so daß Änderungen im betrachteten Produkt $g_{I} \cdot \xi^{2}$ zumeist auf Änderungen in der Korrelationszahl zurückzuführen sind. Es zeigt sich nun, daß bei allen Suspensionen das betrachtete Produkt in der Nähe der Umwandlungstemperatur stark ansteigt und kurz nach der Umwandlungstemperatur ein Maximum besitzt. Oberhalb von $T_{m}$ sinkt der Wert dann wieder mehr oder weniger ab. So ist bei DMPC und DMPC/DPPC 50:50 jeweils ein Maximum bei $25{ }^{\circ} \mathrm{C}$ bzw. $35^{\circ} \mathrm{C}$ zu sehen. Bei der DMPC/DMG 75:25 Suspension sind auch hier zwei ausgeprägte Maxima zu 
entdecken, bei denen $g_{I} \cdot \xi^{2}$ besonders groß ist.

Bemerkenswert ist ferner die Abnahme der Korrelation der Kopfgruppen untereinander bei den DMPC/Cholesterin Suspensionen mit steigendem Cholesteringehalt. Es wird schon seit langem vermutet, daß die Zugabe von Cholesterin die Kooperativität der Lipide beeinträchtigt und vermindert. Dieses zeigt sich unter anderem in cholesterinabhängigen Wärmekapazitätsmessungen [49]. Alle Beobachtungen scheinen mit der Annahme des kritischen Verhaltens bei der Umwandlung übereinzustimmen. Die Membran durchläuft bei der Hauptumwandlung eine Phasenumwandlung zweiter Ordnung. Die Korrelation der lateralen Bewegungen untereinander steigt durch die kritische Verlangsamung und die dadurch entsprechend vergrößerten Korrelationslängen stark an. Die dielektrischen Messungen zeigen einen Geltungsbereich dieser Eigenschaften in gewissem Maße auch für die Orientierung der Kopfgruppen.

Während die DMPC/DPPC 50:50 und die DMPC/DMG 75:25 Mischungen nur aus Lipiden bestehen, müssen die DMPC/Cholesterin Suspensionen getrennt von diesen betrachtet werden, da das Cholesterinmolekül im strengen Sinn kein Lipid ist. Es läßt sich lediglich sehr gut in Membranen lösen. Dabei stellt sich die Frage, wie sich im einzelnen die gelösten Cholesterinmoleküle in der Membran anordnen. Bei Finean [30] und McMullen et al. [102] wird aufgrund von Röntgenstreuung und Kalorimetrie an DMPC/Cholesterin Mischungen die Vermutung angestellt, daß das Cholesterinmolekül eine Wasserstoffbrückenbindung zwischen der (OH)Gruppe des Cholesterins und einem Sauerstoffatom des Glyceringerüstes der Phospholipides eingeht. Abb. 4.22 zeigt schematisch diesen Sachverhalt. Da die Wasserstoffbrückenbindung jedoch direkt unterhalb der polaren Kopfgruppe ansetzt und so das Cholesterinmolekül etwa gleich lang wie die Kohlenstoffketten des Lipides nach unten hin reicht, liegt
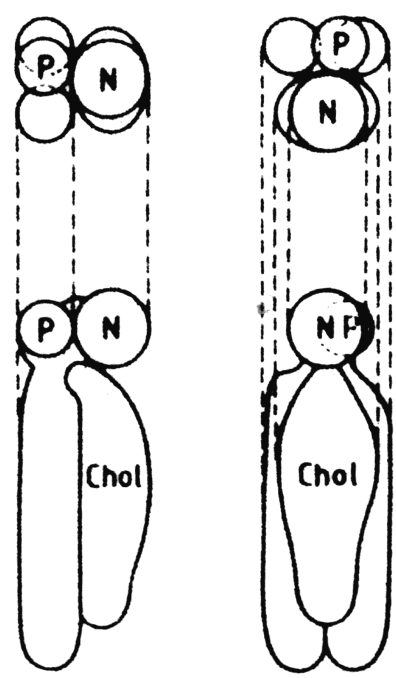

$1 \oplus$

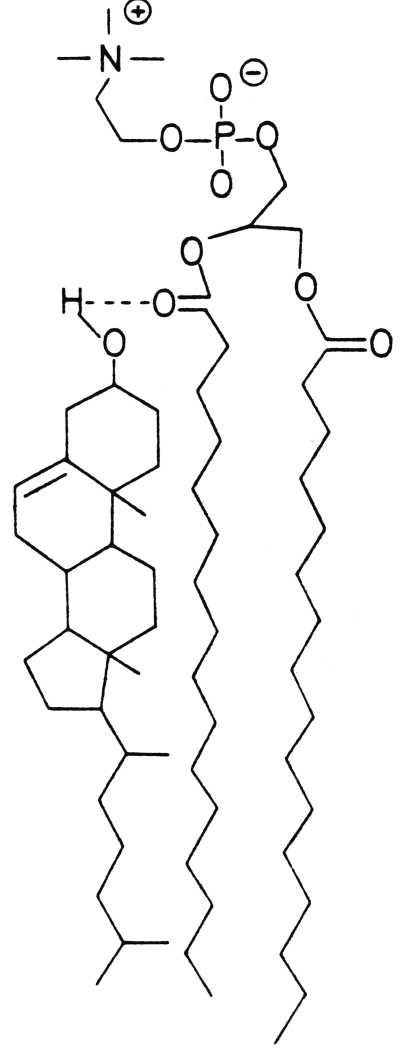

Abb. 4.22: Cholersterin bindet an DMPC der Schluß nahe, daß die Beweglichkeit der Kopfgruppe weitestgehends unbeeinflußt bleibt. Diese Vermutung wird von dem Verlauf des Quotienten $u / \xi^{2}$ in Abb. 4.20 stark gestützt. Der Quotient bzw. die Beweglichkeit der Kopfgruppen bleibt auch bei Zugabe von $30 \%$ Cholesterin unbeeinflußt. Im Rahmen der Meßgenauigkeit ist die Beweglichkeit der Kopfgruppen der Suspensionen mit Zugabe von Cholesterin genauso groß wie die von reinem DMPC.

Aus kalorimetrischen Messungen und aus Kompressibilitätsmessungen (siehe Kap. 3.4) ist bekannt, daß sich die Kooperativität der Umwandlung der Membran bei Zu- 
gabe von Cholesterin zunehmend vermindert. Dies äußert sich u.a. in einer breiteren Verlauf in der Wärmekapazitätskurve bzw. in einem flacheren Verlauf im Temperaturverlauf der Schallgeschwindigkeitszahl (siehe Abb. 3.10). In Abb. 4.21 ist ein deutliches Absinken des Produktes $g_{I} \cdot \xi^{2}$ also die Anzahl der miteinander korrelierten Moleküle zu sehen. Dieses entspricht demnach qualtitativ der Verminderung der Kooperativität der Membran bei Zugabe von Cholesterin.

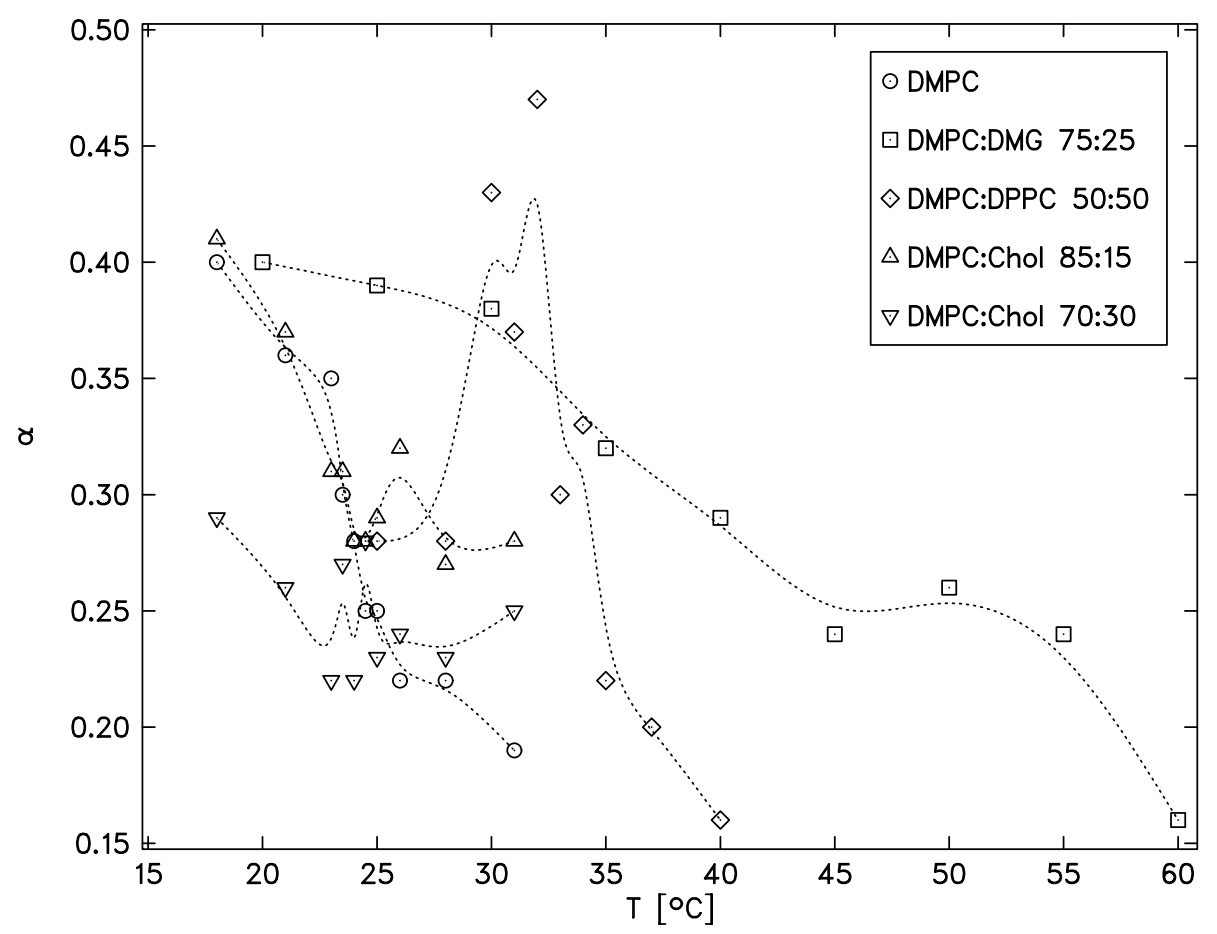

Abb. 4.23: Temperaturverlauf des Verteilungsparameters $\alpha$

Abb. 4.23 zeigt den Verlauf des Verteilungsparameters $\alpha$ aus den Anpassungsrechnungen. Da zwei debyeartige Prozesse im betrachteten Frequenzbereich diskutiert wurden, jedoch nicht ausreichend genau genug angepaßt werden können, muß der Verteilungsparameters $\alpha$ als Maß dafür angesehen werden, wie weit diese beiden Prozesse, falls überhaupt zwei getrennte Relaxationsprozesse vorliegen, im Frequenzbereich auseinander liegen. Es zeigt sich, daß bei allen Suspensionen $\alpha$ von einem relativ hohen Wert von etwa 0,4 auf etwa 0,2 abfällt. Nur die DMPC/DPPC 50:50 Suspension zeigt diesbezüglich ein abnormes Verhalten. Dabei muß jedoch erwähnt werden, daß bei der Anpassung mittels der empirischen Relaxationsfunktionen dieser Verlauf in dieser Ausprägung nicht gefunden wird. Beide Prozesse zeigen jedoch die Tendenz, unterhalb der Umwandlung relativ weit auseinander zu liegen, und oberhalb der Hauptumwandlung fast zu einem debyeartigen Prozeß zu verschmelzen.

Um die Größenordung der Paramter dieser zwei Prozesse zu verdeutlichen ist in Tabelle 4.8 das Ergebnis einer Anpassung einer empirischen Spektralfunktion mit zwei Debye-Termen anstelle des Cole-Cole-Terms zur Beschreibung der Kopfgruppenumlagerung aufgeführt. 


\begin{tabular}{|c|c|}
\hline$\Delta \epsilon_{1}$ & $1,13(9)$ \\
$\tau_{1}[\mathrm{ps}]$ & $542(70)$ \\
$\Delta \epsilon_{2}$ & $2,79(9)$ \\
$\tau_{2}[\mathrm{ps}]$ & $3880(168)$ \\
\hline
\end{tabular}

Tabelle 4.8: Parameter aus der Anpassung einer Spektralfunktion mit zwei Debye-Termen anstelle einer Relaxationszeitverteilung zur Beschreibung der Kopfgruppenumlagerung von DMPC bei $31^{\circ} \mathrm{C}$.

\subsubsection{Die Radien der Vesikel}

Es sei hier noch einmal detailliert auf die Radien der Vesikel, wie sie aus den Anpaßrechnungen gewonnen werden, eingegangen.

Aus Lichtstreumessungen an Lösungen vor DMPC/Wasser Systemen geringer Konzentrationen $\left(<1 \frac{\mathrm{mg}}{\mathrm{ml}}\right)$ ist bekannt, daß die Vesikel bedingt durch den höheren Platzbedarf eines fluiden Lipids, in der Hauptumwandlung quellen und einen leicht größeren Radius bekommen. Die Dimension dieses Vorganges liegt in etwa bei einer Vergrößerung des Radius von 6-7 \%. Dieser Effekt kann somit nicht den dramatischen Anstieg der Radien, wie sie durch die dielektrische Spektroskopie ermittelt werden, erklären. Ein möglicher Erklärungsansatz wäre, wie oben schon erwähnt, in der mangelnden Extrusion

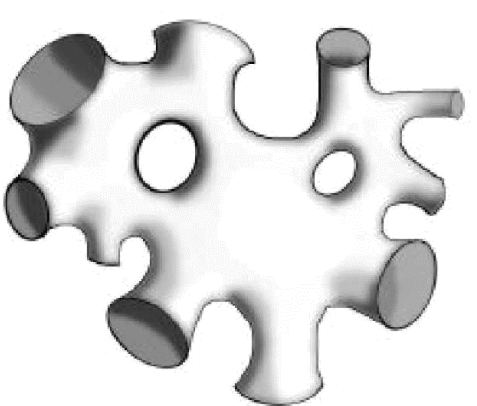

Abb. 4.24: mögliche Struktur der Lipidmembran in der Umwandlung zu suchen. Allerdings könnte auch die vergleichsweise hohe Konzentration von $60 \mathrm{mg} / \mathrm{ml}$ die Ursache sein. Möglicherweise sind Wechselwirkungen zwischen den einzelnen Vesikeln nicht mehr zu vernachlässigen, und es bilden sich in der Umwandlung andere Aggregate als die bisher angenommenen kugelförmigen Strukturen. Denkbar ist hierbei eine Struktur, die als durchgehendes Netzwerk einer Phospholipidmembran bezeichnet wird [55] (Abb. 4.24). Im weiteren ist die Möglichkeit der Fusion der Vesikel gegeben, so daß sich beim Durchfahren der Temperatur aufgrund der extrem hohen Konzentration mit der Zeit größere Vesikel bilden. Verdünnt man eine solche Lösung nach dem Überschreiten der Phasenumwandlung, und mißt den Radius der Vesikel mittels Lichtstreuung, so bekommt man durchaus nicht die relativ homogene Größenverteilung, wie es bei den Konzentration um 2-5 $\frac{\mathrm{mg}}{\mathrm{ml}}$ der Fall ist. Es existieren in einer nicht verschwindenden Konzentration größere Vesikel mit einem Durchmesser von einigen $100 \mathrm{~nm}$ in der verdünnten Lösung.

Mit Hilfe der oben dargelegten Theorie von R. Pottel wird der Radius mittels einer Anpaßrechnung an die dielektrischen Spektren bestimmt. Maßgeblich für die Größe dieses Parameters ist der Unterschied zwischen der Relaxationsstufe reines Wassers und der in den Spektren auftretenden Wasserrelaxationsstufe (siehe Gleichung (4.44)). Um zu zeigen, daß die berechneten Radien keine Artefakte, sondern Ausdruck von real gemessenen Werten sind, ist hier in Abb. 4.25 das Verhältnis der Relaxationsstufe von reinen Wasser zu der Wasserrelaxationsstufe der Lipidsuspensionen aufgetragen. Deutlich ist bei gleicher Konzentration die höhere Absenkung der 
Wasserrelaxationsstufe der DMPC/DPPC 50:50 Suspension gegenüber der DMPC Suspension zu sehen. Somit sind zumindest die berechneten Radien direkt anhand der gemessenen Spektren erklärbar.

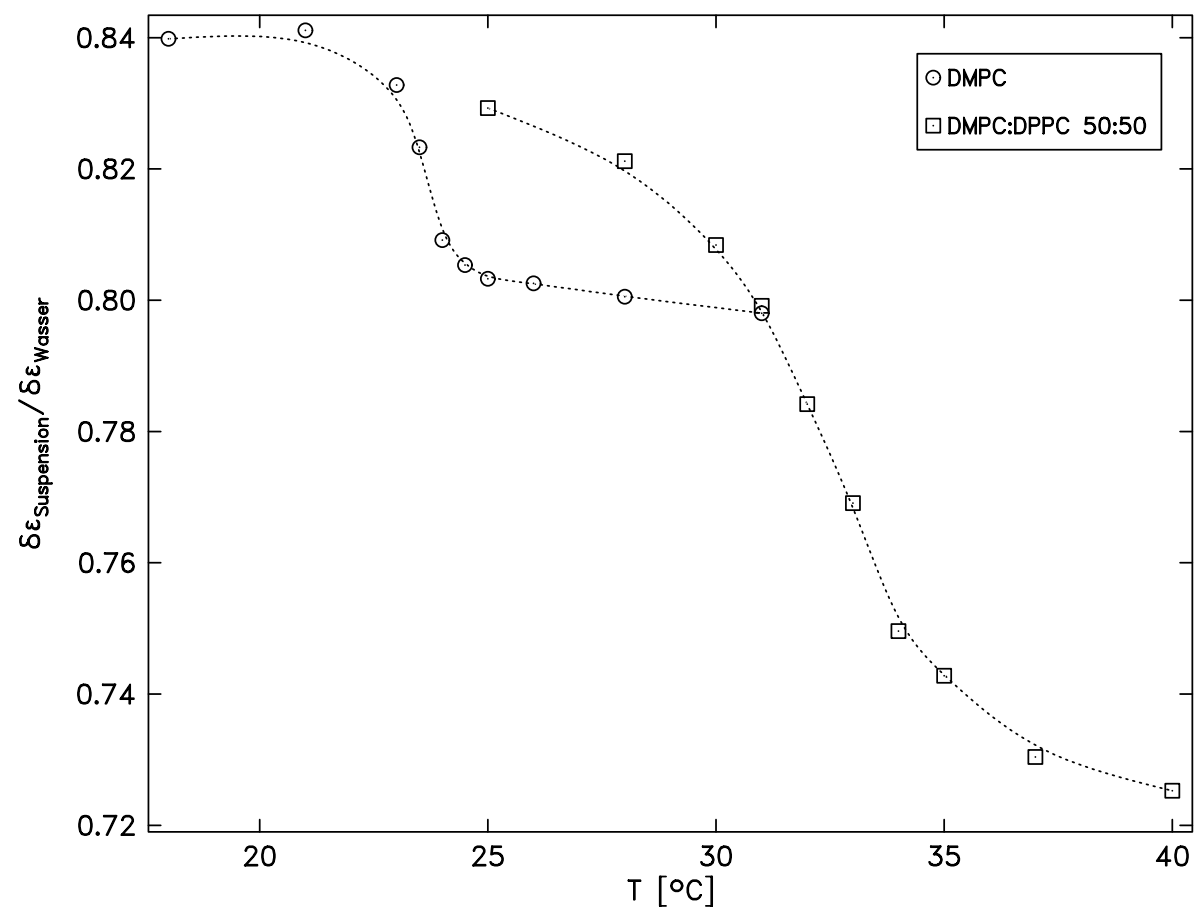

Abb. 4.25: Verhältnis der Relaxationsstufen von reinem Wasser zu der Wasserrelaxationsstufe der Lipidsuspensionen

Bei den hier sehr hohen Konzentrationen kann es zusätzlich zu einer leichten Verformung der Geometrie der Vesikel kommen. Möglicherweise liegen die Vesikel ab einer bestimmten Temperatur nicht mehr in reiner Kugelform vor, sondern bilden Ellipsoide. Da die angewandte Theorie jedoch nur von reinen Kugelschalen ausgeht, führt eine solche Verformung zu einem Fehler, der sich vornehmlich in Gleichung (4.44) bemerkbar macht und somit zu einem scheinbar höheren Radius führen kann. Zum anderen existieren in der Literatur sehr viele Mischungsformeln, die ein Dielektrikum beschreiben, welches aus kugelförmigen Teilchen in einem homogenen Dielektrikum besteht. Es existiert nach wie vor eine gewisse Unsicherheit bei der Wahl der richtigen Mischungsformel, jedoch ist keine entsprechende Formel in der Lage, die sehr ausgeprägte Diskrepanz zwischen der DMPC und DMPC/DPPC VesikelLösung im Bezug auf die Wasserrelaxationsstufe zu erklären (siehe Abb. 4.25). Die Verwendung der Bruggemann Mischungsformel

$$
\epsilon=\epsilon_{w}+\left(\epsilon_{w}-\epsilon_{a}\right)\left[(1-f)\left(\frac{\epsilon}{\epsilon_{w}}\right)^{\frac{1}{3}}-1\right]
$$

bringt im Rahmen der Fehler keine Änderung der Parameter im Vergleich zur Mischungsformel (4.44), was als Bestätigung der in der Theorie verwendeten Mischungsformel betrachtet werden muß.

Abschließend ist noch zu sagen, daß ein geringer Teil des Wassers scheinbar gebunden ist [80] und somit ebenfalls zu einem höheren Radius führen kann. 


\section{$5 \quad$ Grundlagen der Ultraschallspektroskopie}

\subsection{Klassische Dämpfung}

Mit Hilfe der Navier-Stokes-Gleichung und der Kontinuitätsgleichung läßt sich, unter Annahme eines kleinen Wechseldruckes gegenüber dem sonst in dem Medium herrschenden Druck, eine Wellengleichung für den Schalldruck $\tilde{p}$ herleiten [6]. Mit dem Ansatz einer ebenen, harmonischen, gedämpften Welle, die sich in $x$-Richtung ausbreitet, also

$$
\begin{aligned}
& \gamma=\alpha+i \beta \text { und } \beta=2 \pi / \lambda \\
& \tilde{p}=\hat{\tilde{p}} \cdot e^{i \omega t-\gamma x} \quad \text { mit } \quad \lambda=\text { Wellenlänge } \\
& \quad \omega=\text { Kreisfrequenz, }
\end{aligned}
$$

ergibt sich unter der Beachtung der Bedingung $\left((4 / 3) \eta_{s}+\eta_{v}\right) \cdot \omega /\left(\varrho \cdot c_{s}^{2}\right) \ll 1$, für den Dämpfungsexponenten der Schallwelle:

$$
\alpha=\frac{\omega^{2}}{2 c_{s}^{3} \varrho_{o}}\left(\frac{4}{3} \eta_{s}+\eta_{v}\right) \quad \text { mit } \quad c_{s}=\frac{\omega}{\beta}=\text { Schallgeschwindigkeit. }
$$

Die durch die Schallwelle verursachten Druckschwankungen in dem betrachteten Medium führen zu einer Deformation der Volumenelemente des Fluids, die sich einerseits in einer Scherung eines Volumenelementes und einer Volumenänderung niederschlägt. Die Verluste, die die Schallwelle durch diese beiden Effekte widerfährt, ist durch die entsprechenden Viskositäten, die Scherviskosität $\eta_{s}$ und die Volumenviskosität $\eta_{v}$ bedingt.

Im allgemeinen sind $\eta_{s}$ und $\eta_{v}$ veränderlich über der Frequenz. Bei sehr einfachen Flüssigkeiten, wie bei einigen monoatomaren Fluiden, ist jedoch $\eta_{s}$ und $\eta_{v}$ über der Frequenz konstant $\left(\eta_{s}=\eta_{s \infty}\right.$ und $\left.\eta_{v}=\eta_{v \infty}\right)$.

In dem sogenannten B-Wert ist alles zusammengefaßt, was für sehr hohe Frequenzen $\omega \rightarrow \infty$ noch Beiträge liefert.

$$
B=\frac{2 \cdot \pi^{2}}{2 c_{s}^{2} \varrho_{o}}\left(\frac{4}{3} \eta_{s \infty}+\eta_{v \infty}\right)
$$

Gleichung (5.3) wird sehr oft als der klassische Beitrag zur Dämpfung der Schallwelle betrachtet [58] [7].

$$
(\alpha \lambda)_{k l a s s}=B \cdot f
$$

Bhatia [7] führt in seinen Überlegungen im folgenden einen weiteren Beitrag zur klassischen Dämpfung ein, der auf die endliche Wärmeleitfähigkeit zurückgeführt wird. Jedoch kann dieser Term hier gut vernachlässigt werden, da er gegenüber den viskosen Verlusten verschwindend gering ist. Er spielt bei anderen Medien wie z.B. flüssigen Metallen eine Rolle.

Die Dämpfung, verursacht durch in dem Meßbereich beobachteten Relaxationsprozeße, wird als zusätzliche Dämpfung oder Exzeßdämpfung $(\alpha \lambda)_{e x}$ bezeichnet. Diese Zusatzdämpfung wird durch Anpassung von Spektralfunktionen und dem Parameter $B$ an die gemessenen Absorptionsspektren zugänglich:

$$
(\alpha \lambda)_{e x}=(\alpha \lambda)_{g e s}-B \cdot f
$$


Ebenfalls gebräuchlich ist die $\frac{\alpha}{f^{2}}$-Darstellung:

$$
\begin{gathered}
\left(\frac{\alpha}{f^{2}}\right)=\left(\frac{\alpha}{f^{2}}\right)_{e x}+\left(\frac{\alpha}{f^{2}}\right)_{g e s}= \\
\left(\frac{\alpha}{f^{2}}\right)_{e x}=\left(\frac{\alpha}{f^{2}}\right)_{g e s}-\frac{B}{\lambda f}=\left(\frac{\alpha}{f^{2}}\right)_{g e s}-\frac{B}{c_{s}} .
\end{gathered}
$$

\subsection{Zusätzliche Dämpfung}

Besteht die betrachtete Flüssigkeit aus mehr oder wenig komplexen Molekülen, die zwei definierte Zustände einnehmen können und liegt zwischen diesen Zuständen ein Gleichgewicht mit bestimmter Konzentrationsverteilung vor, so führt dies oftmals zu einer Dämpfung, die einen zusätzlichen Betrag zu der oben eingeführten klassischen Dämpfung liefert.

Betrachtet sei nun ein Druck(Temperatur)sprungexperiment, bei dem ein definiert eingestellter Druck auf der Flüssigkeit lastet. Zwischen den zwei möglichen Zuständen der Moleküle wird sich ein bestimmter Gleichgewichtszustand einstellen, der sich jedoch bei plötzlicher Änderung des Druckes in eine bestimmte Richtung verschieben wird. Es stellt sich ein Zustand ein, der den thermodynamischen Parametern entspricht. Im Zeitbereich erhält man ein exponentielles Abklingverhalten mit der Abklingkonstanten $\tau$, der Relaxationszeit, für die Änderung des chemischen Gleichgewichtes von der Konzentration $c$ zu der neuen Gleichgewichtskonzentration $c^{\prime}$.

$$
-\frac{d c}{d t}=\frac{c-c^{\prime}}{\tau}
$$

Eine sich durch das Medium bewegende Schallwelle ist eine Folge von Druckminima bzw. Druckmaxima. Bei Verwendung von harmonischen Wellen $\sim e^{i \omega t}$ ergibt sich unter der Annahme eines exponentiellen Abfalles beim Drucksprungexperiment die Debye Spektralfunktion:

$$
(\alpha \cdot \lambda)\left(\frac{c_{s, \infty}}{c_{s}}\right)^{2}=A \cdot \frac{\omega \tau}{1+(\omega \tau)^{2}},
$$

wobei $c_{s, \infty}=c_{s}(\omega \rightarrow \infty)$ und $A$ die Relaxationsamplitude ist:

$$
A=\frac{\pi \Gamma c_{s, \infty}^{2} \varrho_{o}}{R \cdot T}\left(\frac{\mathcal{A}_{\infty}}{\varrho_{o} \cdot C_{p, \infty}} \cdot \Delta H-\Delta V\right)^{2},
$$

mit

$\begin{array}{rll}R & : & \text { Gaskonstante } \\ T & : & \text { absolute Temperatur } \\ c_{s, \infty}: & \text { Schallgeschwindigkeit für } \omega \gg 1 / \tau \\ \varrho_{o}: & \text { Dichte der Flüssigkeit. }\end{array}$

Der Gamma-Faktor $\Gamma$ wird über Stöchiometriefaktoren $n_{i}$ und die Konzentrationen $c_{i}$ der an dem chemischen Gleichgewicht beteiligten Moleküle bestimmt: 


$$
\frac{1}{\Gamma}=\sum_{i=1}^{m} \frac{n_{i}^{2}}{c_{i}}
$$

Der effektive thermische Ausdehnungskoeffizient bei hohen Frequenzen $\mathcal{A}_{\infty}$ ist gegeben durch:

$$
\mathcal{A}_{\infty}=\left[\frac{1}{V}\left(\frac{\partial V}{\partial T}\right)_{p}\right]_{\omega \rightarrow 0}-\frac{\Gamma \cdot \Delta H \cdot \Delta V}{R \cdot T},
$$

und die effektive Wärmekapazität bei konstantem Druck und für $\omega \gg 1 / \tau$ gilt:

$$
c_{p, \infty}=c_{p, o}-\frac{\Gamma(\Delta H)^{2}}{R \cdot T^{2} \varrho_{o}} .
$$

Die Debye Spektralfunktion beschreibt den Relaxationsvorgang im Frequenzbereich, im Gegensatz zum exponentiellen Abklingen im Zeitbereich. Gemäß der KramersKronig-Relationen führt die zusätzliche Absorption auch zu einer Dispersion der Schallgeschwindigkeit:

$$
\left(\frac{c_{s, \infty}}{c_{s}}\right)^{2}=1+\frac{1}{\pi} A \cdot \frac{1}{1+(\omega \tau)^{2}}
$$

\subsection{Kritisches Verhalten}

Befindet sich eine Flüssigkeit in der Nähe eines kritisches Punktes, so zeigt sie in Bezug auf die Ultraschallabsorption ein gänzlich anderes Verhalten, als es durch die einfache Relaxationsdynamik beschrieben werden kann. Es treten starke Fluktuationen diverser thermodynamischer Parameter auf, insbesondere der Dichte. Die Dichtefluktuationen koppeln an die einfallende Schallwelle und entziehen ihr Energie, was einen zusätzlichen Beitrag zu $(\alpha \lambda)_{e x}$ liefert. Bei der Beschreibung dieser Eigenschaften muß das spezielle Verhalten der Flüssigkeit in der Nähe des kritischen Punktes berücksichtigt werden. Am kritischen Punkt folgt die spezifische Wärme einem Potenzgesetz:

$$
C_{p} \sim\left(\frac{T-T_{c}}{T_{c}}\right)^{-\tilde{\alpha}}
$$

wobei $T_{c}$ die kritische Temperatur darstellt und $\tilde{\alpha}$ als der kritische Exponent der spezifischen Wärme bezeichnet wird. Am kritischen Punkt werden viele Prozesse sehr langsam, insbesondere der Ordnungsparameterfluktuationen, was als "critical slowing down" bezeichnet wird. Einher geht das Anwachsen der Korrelationslänge $\xi$ und der charakteristischen Zeit $\tau$ für das Abklingen der Ordnungsparameterfluktuationen:

$$
\xi=\xi_{0} \cdot\left(\frac{T-T_{c}}{T_{c}}\right)^{-\nu} \quad, \quad \tau \sim\left(\frac{T-T_{c}}{T_{c}}\right)^{-z \nu} .
$$

Dabei wird $z$ als Dynamikexponent und $\nu$ als kritischer Exponent der Korrelationslänge bezeichnet. 


\subsection{Theorie von Bhattacharjee und Ferrell}

Bhattacharjee und Ferrell haben nun eine Theorie zur Beschreibung der Ultraschallabsorption in dreidimensional fluktuierenden binären Flüssigkeiten entwickelt [9] [28] [29] [10] [11]. Zentraler Ausgangspunkt dabei ist die Annahme einer frequenzabhängigen spezifischen Wärme $C_{p}(\omega)$. Die spezifische Größe wird in einen nichtkritischen frequenzunabhängigen Teil $C_{0}$ und einen kritischen Anteil $C(\omega)$ aufgeteilt. Dabei hängt der kritische Anteil der Wärmekapazität in folgender Weise von der reduzierten Temperatur ab:

$$
C_{p}(0) \sim\left(\frac{T-T_{c}}{T_{c}}\right)^{-\tilde{\alpha}}
$$

Wegen der Diffusion, deren Diffusionskonstante durch

$$
D=\frac{k_{B} T}{6 \pi \eta \xi}
$$

gegeben ist, klingen die Konzentrationsfluktuationen mit einer charakteristischen Relaxationsrate ab:

$$
\omega_{B F}(T)=\frac{2 D}{\xi^{2}}=\bar{\gamma}_{0}\left(T-T_{c}\right)^{z \nu}
$$

Bhattacharjee und Ferrell drücken den kritischen Anteil der Wärmekapazität und damit auch die Schallabsorption durch die Relaxationsrate $\omega_{B F}$ aus. Sie bedienen sich dabei der sogenannten dynamische Skalierungshypothese [31] [84] [62] bei der alle Prozesse in der Nähe des kritischen Punktes mit $\omega_{B F}$ skalieren. Mit Hilfe der Renormierungsgruppentheorie und einer $\epsilon$-Entwicklung legen Bhattacharjee und Ferrell schließlich zu einem Ausdruck für die Schallabsorption dar:

$$
\alpha \sim \omega \operatorname{Im}\left(\tilde{C}_{p}(\omega)^{-1}\right) \approx \frac{-a_{0} \pi}{2 z_{0} \nu a} \frac{\omega^{2}}{\left(\operatorname{Re}\left(\tilde{C}_{p}\right)\right)^{2}}\left(\frac{a}{\omega}\right)^{1+a_{0} /\left(z_{0} \nu\right)}
$$

mit

$$
\omega_{B F}=\frac{-i \omega}{a} \quad a=k o n s t
$$

Im weiteren entwickeln die beiden zu einem Ausdruck, der die Absorption nur mittels einer Amplitude und einer Skalierungsfunktion $F(\Omega)$ beschreibt, die nur von der reduzierten Frequenz $\Omega=\omega / \omega_{B F}$ abhängt:

$$
(\alpha \lambda)=A(T) \cdot F(\Omega), \quad \text { mit der Amplitude } \quad A(T)=\hat{A}(T) \cdot \omega_{0}^{-\frac{\tilde{\alpha}}{z \nu}} .
$$

Dabei stellt die Funktion $F(\Omega)$ für diese Arbeit das Hauptergebnis der Untersuchungen dar. Die sogenannte Skalierungsfunktion gibt das Verhältnis der Exzeßdämpfung $(\alpha \lambda)_{e x}$ in der Umgebung um den kritischen Punkt zur Dämpfung am kritischen Punkt selbst $(\alpha \lambda)_{c}$ an:

$$
\frac{(\alpha \lambda)_{e x}}{(\alpha \lambda)_{c}}=F(\Omega)
$$


Bhattacharjee und Ferrell geben in früheren Veröffentlichungen [9] [28] für $F(\Omega)$ ein Integral für die Beschreibung der Absorption an:

$$
F(\Omega)_{(1981)}=\int_{0}^{\infty} \frac{x^{3}}{\left(1+x^{2}\right)^{2}} \frac{K_{B F}(x)}{K_{B F}^{2}(x)+\Omega^{2}} d x
$$

mit

$$
K_{B F}(x)=x^{2}\left(1+x^{2}\right)^{p} .
$$

Der Parameter $\mathrm{p}$ ist abhängig vom System und liegt zwischen null und eins. In späteren Veröffentlichungen [29] wird jedoch eine empirische Skalierungsfunktion angegeben, die die obige Funktion annähert:

$$
F(\Omega)_{(1985)}=\frac{1}{(1+\sqrt{1 / \Omega})^{2}}
$$

Eine Verbesserung stellt auch die folgende empirische Skalierungsfunktion dar [29] [10]:

$$
F(\Omega)_{(1985)}=\frac{1}{\left(1+0,414 \cdot \sqrt{\Omega_{\frac{1}{2}} / \Omega}\right)^{2}} \quad \text { mit } \Omega_{\frac{1}{2}} \approx 2.1
$$

In [11] berechnen Bhattacharjee und Ferrell schließlich eine Skalierungsfunktion für einen Flüssigkristall in der Umgebung eines Phasenüberganges, wie er auch bei den Membranen aus Lipiden vorkommt:

$$
F(\Omega)_{(1997)}=\sqrt{\frac{2}{\Omega}} \cdot\left((1+\Omega)^{1 / 4} \cdot \cos \left(\frac{1}{2} \arctan \Omega\right)-1\right) .
$$

Alle diese Skalierungsfunktionen haben die gleiche spektrale Form. Die letzte Form ist die Funktion, wie sie in den folgenden Berechnungen zur Anpassung an die gewonnenen Ultraschallspektren verwendet wird. An die Spektren wird also die Funktion

$$
(\alpha \lambda)_{e x}=A_{B F}(T) F(\Omega) f^{-\frac{\tilde{\alpha}}{z \nu}},
$$

mit der Skalierungsfunktion (5.28) angepaßt. Freie Parameter dabei sind die Amplitude $A_{B F}(T)$ und die charakteristische Relaxationsrate $\omega_{B F}$.

In der Theorie von Bhattacharjee und Ferrell spielen, wie oben gesehen, kritische Exponenten eine entscheidende Rolle. Die realen Zahlenwerte dieser Konstanten sind durch die Dimensionalität festgelegt. Die Bhattacharjee-Ferrell-Theorie gilt für dreidimensionale, binäre Flüssigkeiten in der Nähe des kritischen Entmischungspunktes. Die drei hier auftretenden kritischen Exponenten sind:

$$
\tilde{\alpha}=0.11 \quad z=3.05 \quad \nu=0.63 .
$$

Mittels diverser thermodynamischer Größen, läßt sich auch die Amplitude aus (5.29) analytisch darstellen. Am kritischen Punkte gilt:

$$
\left(\frac{\alpha}{f^{2}}\right)_{c}=\frac{\pi^{2} \delta \Delta C}{2 T_{c}}\left(\frac{\Omega_{\frac{1}{2}} \omega_{0}}{2 \pi}\right)^{\delta} \cdot \frac{c_{s} g^{2}}{C_{p}^{2}} \cdot f^{-(1+\delta)}+B
$$


Dabei ist $\delta=\frac{\tilde{\alpha}}{z \cdot \nu}=0,06$ und $c_{s}$ die Schallgeschwindigkeit bei $T_{c}$. g ist ein Kopplungsfaktor mit

$$
g=\rho_{c} C_{p}\left(\frac{d T_{c}}{d P}-\frac{T \alpha_{p}}{\rho C_{p}}\right) .
$$

$\rho_{c}$ ist die Dichte am kritischen Punkt und $\alpha_{p}$ der thermische Expansionskoeffizient. Die Größen $\Delta C, C_{p}$ und $C_{0}$ hängen mit dem kritischen Exponenten der Wärmekapazität wie folgt zusammen:

$$
C_{p}=\Delta C \cdot \hat{t}^{-\tilde{\alpha}}+C_{0} .
$$

Dabei ist $\hat{t}$ die reduzierte Temperatur $\hat{t}=\frac{\left|T-T_{c}\right|}{T_{c}}$.

\subsection{Andere Modelle}

Es soll an dieser Stelle nicht verschwiegen werden, daß es weitere Modelle für das kritische Verhalten der Ultraschallabsorption gibt:

Das Modell von Romanov und Solojev: 1965 wird ein Modell zur Ultraschallabsorption entwickelt, welches die Absorption durch Konzentrationsfluktuationen in binäre Flüssigkeiten, die sich nicht in der Nähe eines kritischen Punktes befinden, beschreibt [122] [123]. Dabei werden wie bei Bhattacharjee und Ferrell nicht die einzelnen molekularen Prozesse, sondern die Veränderungen der thermodynamischen Größen unter der Voraussetzung, daß die Dynamik der Fluktuationen durch Diffusion bedingt ist, betrachtet.

Diese Modell kommt offensichtlich für die Systeme der Lipidmembranen nicht in Betracht, da es nur außerhalb des kritischen Punktes Gültigkeit besitzt.

Das Modell von Fixman: Fixman entwickelt in den 60er Jahen ein Modell, welches auch für die Nähe des kritischen Punktes konzipiert ist. Die Theorie ist sowohl für das Verhalten binärer Mischungen als auch für den flüssig-gasförmigen Übergang anwendbar [33] [32] [34]. Fixmans Idee ist es, die Absorption auf die Relaxation der spezifischen Wärme zurückzuführen. Es fließen erste Grundgedanken der Modenkopplungstheorie ein, bei der in kritischen Systemen die bei nichtkritischen Systemen verwendete Linearisierungen der hydrodynamischen Gleichungen nicht mehr zulässig sind. Die Folge ist ein Kopplung der Moden, wie z.B. klassisch gedämpfter Schallwellen und von Teilchen- oder Wärmediffusion. Hier sei nun noch das für die Ultraschallspektroskopie wichtige Ergebnis aufgeführt:

$$
(\alpha \lambda)=\hat{A}_{F} \int_{0}^{\infty} \frac{x^{2}}{1+x^{2}} \frac{\Omega_{F} x^{2}}{\left(x^{2}\left(1+x^{2}\right)\right)^{2}+\Omega_{F}} d x .
$$

Das Modell von Kawasaki: Kawasaki erweitert die Theorie von Fixman Ende der 60er Jahre [87] und enthält die Fixman-Theorie als Grenzfall. Berücksichtigt wird die komplexe Viskosität. Die schon erwähne Modenkopplungstheorie wird auf die Schallabsorption angewandt: 


$$
(\alpha \lambda)=\hat{A}_{K} \int_{0}^{\infty} \frac{x^{2}}{\left(1+x^{2}\right)^{2}} \frac{K(x)}{K^{2}(x)+\Omega^{2}}
$$

mit der Kawasaki-Amplitude $\hat{A_{K}}$, der reduzierten Frequenz $\Omega=\omega / \omega_{D}$ und der Kawasaki-Funktion

$$
K(x)=3 / 4\left(1+x^{2}+\left(x^{3}-1 / x\right) \arctan x\right) .
$$

Das Modell von Kroll und Ruhland: Diese Modell wird Anfang der 80er Jahre entwickelt und stützt sich auf die Anwendung der dynamischen Renormierungsgruppentheorie und der $\epsilon$-Entwicklung [91] [92]. Es wird ein memoryfunktion-Mechanismus eingeführt, der es erlaubt, die Absorption und Dispersion als Funktion der durch die Reihenentwicklung gewonnenen Volumenviskosität auszudrücken. Das Endergebnis ist fast analog zum Modell von Bhattacharjee und Ferrell und unterscheidet sich lediglich ein wenig in der Skalenfunktion.

Das Modell von Folk und Moser: 1998 stellen Folk und Moser eine weitere Theorie zur Beschreibung des Einflusses von Konzentrationsfluktuationen auf die Ultraschallabsorption vor [37] [36]. Leider geben sie keine geschlossene Form für die Absorption einer Schallwelle an. In [37] werden jedoch die Ergebnisse der Überlegungen mit der Theorie von Bhattacharjee und Ferrell verglichen. Dabei fällt auf, daß sich die dort angegebenen Skalierungsfunktionen nur in sehr geringem Maße voneinander unterscheiden. Im Rahmen der Meßgenauigkeit der in dieser Arbeit vorgestellten Ultraschallspektrum wäre ein solcher Unterschied kaum feststellbar (siehe auch Kapitel 7.2.2).

Zusammenfassend läßt sich sagen, daß unter allen hier erwähnten Theorieren der Theorie von Bhattacharjee und Ferrell der Vorzug gewährt wird. Lediglich der Fixman-Kawasaki Theorie wird zusätzliche Aufmerksamkeit gewidmet. Allerdings zeigen Versuche der Anpassung der entsprechenden Spektralfunktionen an die Ultraschallspektren, daß die Theorie von Bhattacharjee und Ferrell die Spektren in weiten Teilen besser beschreibt als die Fixman-Kawasaki Theorie [48].

\subsubsection{Die Beziehung zwischen den Modellen}

Tanaka et al. [138] zeigt, daß trotz der unterschiedlichen Ansätze in den Modellen von Fixman/Kawasaki, Kroll und Ruhland und Bhattacharjee und Ferrell die Ursache für die Absorption bei allen Modellen in der zeitlichen Korrelation der Fluktuationen der inneren Energie zu suchen ist. Sie sind somit nach einigen Änderungen in der Lage, eine allgemein gütige Amplitude anzugeben:

$$
\hat{A}=\frac{\pi g^{2} c_{s}^{2} \tilde{A}}{T \tilde{B}^{2}}
$$

Dabei sind $\tilde{A}$ und $\tilde{B}$ Parameter, die durch folgende Beziehung definiert werden:

$$
c_{p}=\tilde{A} \tilde{t}^{-\tilde{\alpha}}+\tilde{B}
$$


$\mathrm{g}$ ist eine dimensionslose Konstante, die wie folgt definiert ist:

$$
g=-\rho_{k r} c_{p}\left(\frac{d T_{c}}{d P}-\frac{T \alpha_{p}}{\rho c_{p}}\right)=\tilde{B} \tilde{C}_{0} T_{c} / \tilde{A} \tilde{C}_{1} T .
$$

Dabei ist $\rho_{k r}$ die Dichte am kritischen Punkte und die Parameter $\tilde{C}_{0}$ und $\tilde{C}_{1}$ sind mit dem thermischen Ausdehnungskoeffizieten verknüpft:

$$
\alpha_{p}=\tilde{C}_{0} \tilde{t}^{-\tilde{\alpha}}+\tilde{C}_{1}
$$

\subsubsection{Anwendbarkeit des Modells auf Lipidsysteme}

Es stellt sich an dieser Stelle die Frage, ob das Modell von Bhattacharjee und Ferrell überhaupt auf die hier behandelten Lipidsuspensionen anwendbar ist. Alle oben aufgeführten Modelle dienen der Beschreibung der Ultraschalldämpfung von binären Systemem, die sich bei einer kritischen Temperatur entmischen. Somit ist sofort klar, daß auch das Modell von Bhattacharjee und Ferrell von einem unendlich ausgedehntem, dreidimensional fluktuierenden Kontinuum ausgeht. Diese Voraussetzungen werden auf den ersten Blick für die Lipidsysteme nicht erfüllt. Zunächst gilt es sich klar zu machen, auf welche Weise die Ultraschallwelle an die Membranen koppelt. V. Ivanonva kann Relaxationszeiten bei DMPC/Wasser Systemen nachweisen, die im Bereich von einigen Sekunden liegen [67], und ordnet diesen Effekt dem Temperaturausgleich zwischen Membran und suspendierenden Wasser zu. Da eine Ultraschallwelle sich aus periodischen Dichte und Temperaturschwankungen zusammensetzt (siehe Gleichung (5.1)), kann der Wärmeaustausch zwischen Membran und Wasser bei den hier betrachteten Frequenzen (>150 kHz) vernachlässigt werden. Zudem geht die Theorie von T. Heimburg [53] ebenfalls von dieser Tatsache aus. Die gute Übereinstimmung der umgerechneten Wärmekapazitätskurven in entsprechende Schallgeschwindigkeitskurven, die bei ca. $2 \mathrm{MHz}$ aufgenommen werden, unterstützt diese Vermutung. Die im Ultraschallexperiment vorkommenden Wellenlängen $\lambda=c_{s} / f$ mit $c_{s} \approx 1500 \mathrm{~m} / \mathrm{s}$ reichen von $\lambda \approx 8 \cdot 10^{-3} \mathrm{~m}$ bis $\lambda \approx 8 \cdot 10^{-7}$ und liegen damit im ungünstigsten Fall immer noch eine Größenordung über dem Vesikeldurchmesser. Somit kann als gesichert angesehen werden, daß die Ultraschallwelle ausschließlich über die Druckschwankungen an die Vesikel koppelt. Die Lipidsuspension muß somit als binäres System bestehend aus einem relativ hohem Wasseranteil, der lediglich die Aufgabe hat, die Druckschwankungen auf die Membranen weiterzugeben, und einem je nach Temperatur und Frequenz mehr oder wenig kompressiblen Vesikelanteil angesehen werden. Die dabei in der Membran entstehende Wärme kann bei den hier verwendeten Frequenzen als in der Membran verbleibend betrachtet werden, und aufgrund dessen kann die Membran als völlig losgelöst vom umgebenden Wasser betrachtet werden. Die Theorie von Bhattacharjee und Ferrell ist somit möglicherweise anwendbar, da das gesamte Wasser/Lipid System sich wegen der großen Wellenlängen makroskopisch scheinbar dreidimensional verhält. Letztendlich kann jedoch nur eine konkrete Überprüfung, beispielweise der zum System gehörenden kritischen Exponenten $\tilde{\alpha}, z$ und $\nu$ oder das Verhalten der Ultraschalldämpfung speziell am kritischen Punkt $T_{m}$, Aufschluß über die Gültigkeit des Modells von Bhattacharjee und Ferrell geben. 


\section{Meßverfahren der Ultraschallspektroskopie}

Untersuchungsgegenstand in der Ultraschallspektroskopie sind im allgemeinen durch inter- und intramolekulare Vorgänge verursachte Relaxationsprozesse, die im Gegensatz zu Resonanzphänomenen breite Spektren über mehr als eine Dekade im Frequenzbereich aufweisen. Um die sie charakterisierenden Größen wie Relaxationsstufen und Relaxationszeiten möglichst genau bestimmten zu können, ist es erforderlich, den Dämpfungsexponenten oder die Schallgeschwindigkeit über einen großen Frequenzbereich zu messen. Meistens werden Temperatur und Konzentration der Meßlösung variiert, da die alleinige Bestimmung eines Ultraschallspektrums zu unspezifisch für eine genaue Zuordnung der Relaxationsprozesse zu den molekularen Vorgängen ist. Mit den in dieser Arbeit verwendeten Apparaturen kann ein Frequenzbereich von $200 \mathrm{kHz}$ bis $5 \mathrm{GHz}$ ausgemessen werden.

Neben den Relaxationsprozessen, die zur Dämpfung der Ultraschallwelle beitragen, enthält der Dämpfungsexponent $\alpha$ ferner den asymptotisch, hochfrequenten Beitrag, der mit dem Quadrat der Frequenz ansteigt (Gleichung (5.2)). Dies führt bei den hier überstrichenen Frequenzen zu höchst unterschiedlichen Dämpfungen in der Flüssigkeit. So ergeben sich Dämfungswerte von $\alpha=2 \cdot 10^{-3} \mathrm{~m}^{-1}$ und $\alpha=1 \cdot 10^{6} \mathrm{~m}^{-1}$. Dieser Sachverhalt macht es nötig, im betrachteten Frequenzbereich mit zwei verschiedenen Meßverfahren zu arbeiten: dem Resonatorverfahren und dem Schwingungspulstransmissionsverfahren.

Oberhalb von ca. $10 \mathrm{MHz}$ ist es möglich, mit dem Schwingungspulstransmissionsverfahren [76] [77] [81] zu messen. Dabei wird der Dämpfungsexponent aus dem Amplitudenabfall der Schallwelle längs einer variablen Meßstrecke bestimmt. Um eine genügend große Genauigkeit zu erreichen, muß die Länge der Meßstrecke so gewählt werden, daß der Amplitudenabfall mindestens $2 \mathrm{~dB}$ beträgt. Jedoch sind diesem Verfahren durch die Notwendigkeit zur Thermostatierung der Meßzellen und der nur endlichen Verfügbarkeit der Meßflüssigkeit Grenzen gesetzt. Deshalb verwendet man bei Frequenzen unterhalb von ca. $15 \mathrm{MHz}$ das Resonatorverfahren [23] [25] [72], bei dem durch Mehrfachreflexion in der Meßzelle die Schallwelle gefaltet wird und somit das benötigte Volumen vergleichsweise klein gehalten werden kann. Bei bestimmten Frequenzen entsteht im Resonator ein Stehwellenfeld aus dessen zugehöriger Resonanzfunktion die Dämpfung der im Resonator befindlichen Flüssigkeit bestimmt werden kann.

\subsection{Das Resonatorverfahren}

\subsubsection{Meßprinzip}

Es wird zunächst ein idealer Resonator bestehend aus zwei schallharten, zueinander parallelen Schallwandlern, die im Abstand 1 angeordnet sind und deren Radien als unendlich angenommen werden, betrachtet. Der sendende Schallwandler strahlt nun Schallwellen in die zwischen den Wandlern befindliche Flüssigkeit und die Schallwelle wird daraufhin mehrfach an beiden Wandlern hin- und herreflextiert. Der resultierende Druck $p_{e}$ am Empfangswandler ergibt sich somit durch Überlagerung aller Teilwellen zwischen den Wandlern. Mit der Schallgeschwindigkeit $c_{s}$ und der 
komplexen Ausbreitungskonstanten $\gamma=\alpha+i(2 \pi / \lambda)^{-1}=\alpha+i 2 \pi f / c_{s}$ erhält man:

$$
\begin{gathered}
p_{e} \sim(1+r) e^{-\gamma l} e^{i 2 \pi f t}+(1+r) r^{2} e^{-3 \gamma l} e^{i 2 \pi f t}+(1+r) r^{4} e^{-5 \gamma l} e^{i 2 \pi f t}+\ldots \\
p_{e} \sim \frac{e^{-\gamma l} e^{i 2 \pi f t}}{1-r^{2} e^{-2 \gamma l}} .
\end{gathered}
$$

Setzt man an der Flüssigkeit/Wandler-Grenzschicht einen Reflexionsfaktor $|r|=1$ voraus, so ergibt sich für die Schalldruckamplitude $\hat{p}_{e}$ :

$$
\hat{p}_{e} \sim \frac{1}{\sinh (\gamma l)}=\frac{1}{\sinh (\alpha l) \cos \left(\frac{2 \pi l}{c_{s}} f\right)+i \cdot \cosh (\alpha l) \sin \left(\frac{2 \pi l}{c_{s}} f\right)} .
$$

Gleichung (6.3) beschreibt die Übertragungsfunktion des Resonators. Die auftretenden Resonanzfrequenzen korrespondieren mit maximalem Druck am Empfangswandler. Somit folgt:

$$
f_{n}=n \frac{c_{s}}{2 l}
$$

Abweichend vom ideal biplanaren Resonator ergibt sich für die Resonanzfrequenzen bei konkaver Geometrie der Wandler [3]:

$$
f_{n}=\left(n+\frac{\arccos g}{\pi}\right) \frac{c_{s}}{2 l}
$$

Sollten beide Schallwandler konkav gekrümmt sein, so gilt mit dem Krümmungsradius $K$, daß $g=1-l / K$. Ist nur einer der beiden Wandler gekrümmt, so gilt alternativ $g=\sqrt{1-l / K}$. Wie man sieht, ergeben sich auch bei einer konkaven Geometrie idealerweise Resonanzfrequenzen mit konstantem Frequenzabstand.

Im Gegensatz zum idealen Resonator, besitzt die Schallwelle im realen Resonator eine endliche Eindringtiefe in die Schallwandler. Der Impedanzsprung an der Grenzschicht Quarzwandler-Luft kann als nahezu ideal schallweich angesehen werden, wohingegen sich die Impedanz Flüssigkeit-Wandler je nach Frequenz des Signals derart ändert, daß die Schallwelle eine mehr oder weniger große Eindringtiefe in den Wandler widerfährt. Somit ergibt sich eine größere effektive Zellänge $l$, was sich natürlich auf die Lage der Resonanzen auswirkt. Aus der Theorie der Leitungsgleichungen [105] läßt sich bestimmen, wie weit die Schallwelle in den Quarz eindringt. Es zeigt sich, daß in der Nähre der Wandlerresonanz die Eindringtiefe besonders hoch ist, und zwischen zwei aufeinanderfolgenden Wandlerresonanzen in guter Näherung eine schallharte Reflexion an der Wandlerinnenseite angenommen werden kann. Im Hinblick auf die Bestimmung der Flüssigkeitsdämpfung kann die Dämpfung der Schallwelle innerhalb des Wandlers vernachlässigt werden.

Für kleine Dämpfungen $(\alpha l \ll 1)$ läßt sich Gleichung (6.3) um eine Resonanzfrequenz linearisieren und man erhält eine einfachen Beziehung zwischen Halbwertsbreite der Resonanzkurve und der Dämpfung:

$$
\alpha=\frac{\pi}{c_{s}} \Delta f
$$

Für die Güte folgt weiter:

$$
Q=f / \Delta f
$$

Im realen Resonator existieren neben den Verlusten in der Flüssigkeit auch noch apparative Verluste, die durch die Nichtidealität der Anordnung und der Bauteile bedingt sind: 
- Ein Teil der Schallenergie wird an der Luft zugewandten Seite der Wandler durch den nicht idealen schallweichen Impedanzsprung $(|r|<1)$ abgestrahlt.

- Die elektro-akustische Ankopplung kann mangelhaft sein.

- Es tritt zunehmend Beugung der Schallwelle an der Berandung des Resonator bei tiefen Frequenzen auf. Daraus resultiert eine zunehmende Abstrahlung von Schall durch die Halterung der Schallwandler.

Bei nicht zu hoher Dämpfung kann man eine lineare Überlagerung von apparativen Verlusten und den Verlusten innerhalb der Flüssigkeit annehmen. Somit ergibt sich die insgesamt resultierende Dämpfung als einfache Addition:

$$
(\alpha \lambda)_{\text {gemessen }}=(\alpha \lambda)_{\mathrm{fl}}+(\text { Dämpfung })_{\mathrm{app}} \quad .
$$

Die apparativen Verluste werden mittels eine Messungen einer Flüssigkeit bekannter Dämpfung bestimmt. Dabei sollten zusätzlich andere Eigenschaften der Referenzflüssigkeit, auf die weiter unten eingegangen wird, entsprechend zu den Eigenschaften der eigentlich zu messenden Flüssigkeit gewählt werden.

\section{Beugungsverluste}

Labhardt [94] untersucht die Beugungsverluste in einer plan-planen Anordnung. Danach lassen sich die Beugungsverluste wie folgt berechnen:

$$
\begin{aligned}
&(\alpha \lambda)_{\text {beug }}=\frac{0.147}{\beta_{2}}\left(\frac{c_{s}}{R f_{n}}\right)^{2}, \text { wobei } \\
& \beta_{2}=\frac{c_{s} \varrho_{s}}{c_{w} \varrho_{w}}: \text { spezifische Impedanz der seitlichen Zellberandung } \\
& R \quad: \text { Radius der seitlichen Zellwand } \\
& c_{w}: \text { Schallgeschwindigkeit der seitlichen Zellwand } \\
& \varrho_{w}: \text { Dichte der seitlichen Zellwand } \\
& \varrho_{s}: \text { Dichte der Flüssigkeit . }
\end{aligned}
$$

Demnach steihen die Verluste mit dem Quadrat der Schallgeschwindigkeit an. Deshalb sollte bei Verwendung einer Referenz die Schallgeschwindigkeit mit der Meßlösung möglichst gut übereinstimmen. Wegen der Diche in $\beta_{2}$ sollten möglichst auch die Dichten der Referenzflüssigkeit mit $\rho_{s}$ vergleichbar sein. Beides ist bei den hier betrachteten Lipidsuspensionen und der Referenzflüssigkeit Wasser sehr gut erfüllt.

\subsubsection{Bestimmung des Dämpfungsexponenten}

Die gemessene Übertragungsfunktion enthält neben den schon beschriebenen Eigenschaften der Flüssigkeit und des Resonators weitere Beiträge, die zur gesamten Übertragungsfunktion beitragen:

- Die Quarzwandler sind ein resonanzfähiges System, welches somit zur Frequenzabhängigkeit der Gesamtfunktion beiträgt. 
- Es sind neben den Hauptmoden radiale Nebenmoden anregbar.

- Durch die Antenneneigenschaften der Schallwandler kann es zu elektrischem Übersprechen kommen.

Diese Beiträge sind leider nicht zu vernachlässigen. Sie tragen zum Teil erheblich zur Störung der Hauptmoden bei und müssen deshalb bei den Anpaßrechnungen zur Bestimmung der Dämpfung $\alpha$ berücksichtigt werden.

\section{Nebenmoden}

Aufgrund der speziellen radialen Geometrie des Resonators, der im Gegensatz zum idealen Resonator, natürlich nur eine endliche Ausdehnung in radialer Richtung besitzt, ergeben sich Resonanzbedingungen für die Zelle, die zur Ausbildung von Moden höherer Ordnung führen. Die Resonanzfrequenzen dieser zusätzlichen Moden schließen im Frequenzbereich oberhalb der Hauptmode an. Es handelt sich dabei um Moden, welche neben den Wandlern parallelen Knotenflächen zusätzliche radiale Knotenflächen aufweisen. Für die Resonanzfrequenz der $j$-ten Nebenmode der $n$-ten Hauptmode gilt für einen Resonator mit plan-planer Geometrie [93]:

$$
f_{n, j}=f_{n}+\frac{c_{s}^{2}}{8 R^{2}} \frac{(j-1 / 4)^{2}-(3 / 4)^{2}}{f_{n}} .
$$

Bei einem Resonator mit plan-konkaver Geometrie liegt eine andere Nebenmodenverteilung vor als beim plan-planen Resonator. Die Nebenmoden besitzen hier einen äquidistanten Abstand zur Hauptmode:

$$
f_{n, j}=f_{n}+j \frac{c_{s}}{\pi l} \arccos \sqrt{1-l / K} .
$$

Mit zunehmender Flüssigkeitsdämpfung verbreitert sich die Resonanzkurve sowohl der Hauptmode als auch der dazugehörigen Nebenmoden derart, daß sie sich gegenseitig überlagern und so die Übertragungsfunktion der Hauptmode erheblich stören. Dieses ist besonders im oberen Frequenzbereich der Resonatoren der Fall, wo allein schon durch die klassische Dämpfung die Verbreiterung der Moden so groß ist, daß grundsätzlich eine Beeinflussung der Hauptmoden vorliegt. Um den Einfluß der Nebenmoden von den Eigenschaften der Hauptmode zu trennen muß bei der Anpassung von Gleichung (6.3) die Übertragungsfunktion der Nebenmode mit berücksichtigt werden. Es wird nun eine Übertragungsfunktion $M(f)$ bestehend aus einer Überlagerung von der Hauptmode und $N-1$ Nebenmoden verwendet:

$$
M(f)=\sum_{j=1}^{N} \frac{A_{j}}{\sinh \left(\alpha_{j} l\right) \cos \left(\frac{2 \pi l}{c_{s}}\left(f-f_{n, j}\right)\right)+i \cdot \cosh \left(\alpha_{j} l\right) \sin \left(\frac{2 \pi l}{c_{s}}\left(f-f_{n, j}\right)\right)},
$$

mit den Bezeichnungen

$j=1:$ Index der Hauptmode,

$j=2 \ldots N \quad:$ Indizes von N-1 Nebenmoden,

$A_{j}:$ Amplitude der j-ten Resonanz,

$f_{n, j}$ : Resonanzfrequenz der j-ten Resonanz zur Hauptmode n,

$\alpha_{j}:$ Dämpfungswert zur j-ten Resonanz. 
Somit erhält man für jede Mode drei anzupassende Parameter $A_{j}, \alpha_{j}$, und $f_{n, j}$. In Abb. 6.1 ist ein Beispiel für eine solche Anpassung an die gemessene Übertragungsfunktion mit einer Hauptmode und zwei Nebenmoden dargestellt.

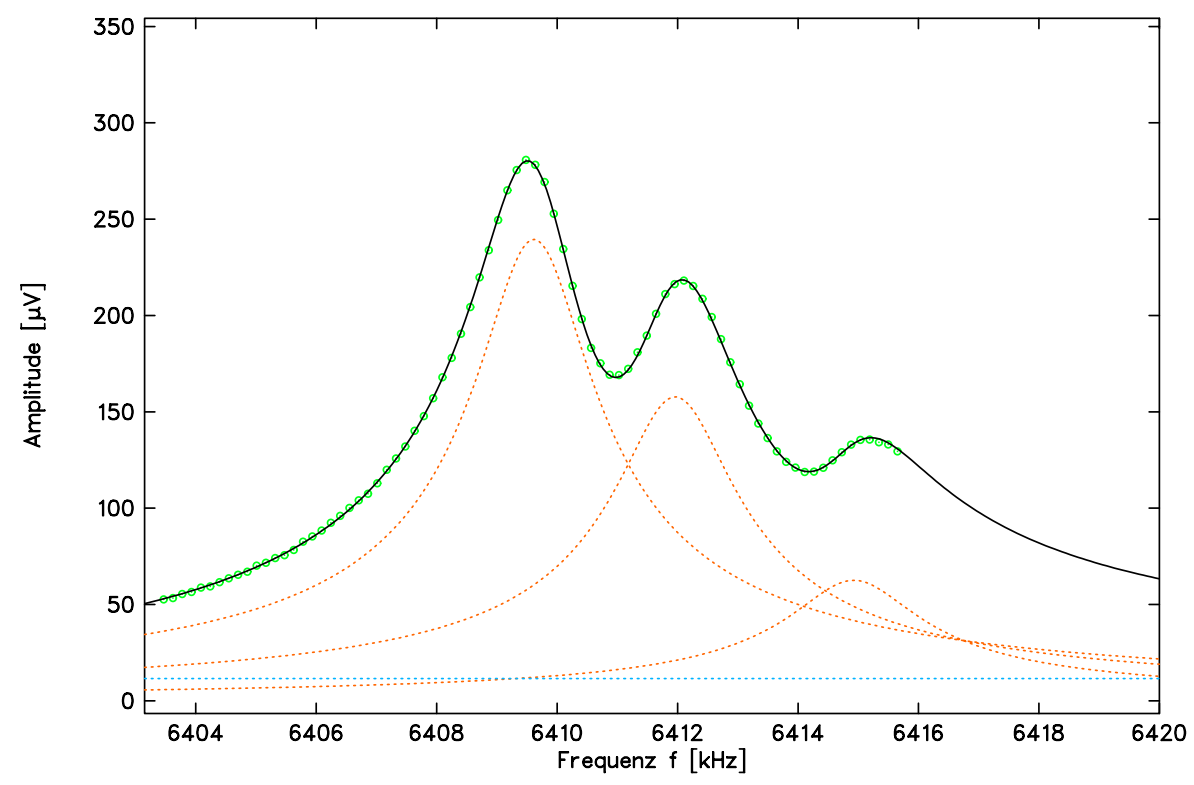

Abb. 6.1: Gemessene Amplitude um den Bereich einer Hauptmode bei $6,4 \mathrm{MHz}$ des plan-planen Resonators mit einer DMPC/Wasser 2 $\mathrm{mg} / \mathrm{ml}$ Füllung bei $25^{\circ} \mathrm{C}$. Gestrichelte Kurven: Betragsfunktion der Hauptmode und zweier Nebenmoden; durchgezogene Linie: Betragsfunktion der komplexwertigen Summe aller angepaßten Moden. Die horizontale gestrichelte Linie zeigt das mitangepaßte elektrische Übersprechen.

\section{Elektrisches Übersprechen}

Eine weitere Störung der Übertragungsfunktion kann durch das elektrische Übersprechen zwischen beiden Wandlern entstehen. Jedoch ändert sich dieser Effekt in den betrachteten Frequenzbereichen kaum, so daß es gut in die Anpassung mit einfließen kann. Dazu wird die normierte Übertragungsfunktion $M(f)$ um eine komplexe Konstante erweitert, also einen Amplitudenparameter $A_{u}$ und einen Phasenparameter $\phi_{u}$. Somit ergibt sich für die anzupassende Übertragungsfunktion $\tilde{M}(f)$ :

$$
\tilde{M}(f)=M(f)+A_{u} e^{i \phi_{u}}
$$

\section{Korrektur nach Kononenko}

Kononenko zeigte, daß neben dem Einfluß der Dichte und der Schallgeschwindigkeit auf die Flüssigkeitsdämpfung in geringem Maße die Dämpfung selbst die apparativen Verluste beeinflußt. Wie groß die Verluste der Schallwelle in den Wandlern selbst sind, hängt von der Dämpfung in der Flüssigkeit selbst ab. Damit Gleichung (6.8) 
auch weiterhin gilt, muß die gemessene Dämpfung gemäß [88] korrigiert werden:

$$
\begin{gathered}
(\alpha \lambda)_{k o r}=(\alpha \lambda)\left(1+a \mu\left(1-\frac{\pi f}{\alpha c_{s} Q_{q}}\right)\right), \text { wobei } \\
a=2 \frac{c_{s} l_{q}}{c_{q} l}, \quad \mu=\frac{1}{\gamma \cos ^{2}\left(\pi f / f_{q}\right)+\gamma^{-1} \sin ^{2}\left(\pi f / f_{q}\right)}, \quad \gamma=\frac{\rho_{s} c_{s}}{\rho_{q} c_{q}}
\end{gathered}
$$

mit der Dichte von Quarz $\rho_{q}$ beziehungsweise der Meßflüssigkeit $\rho_{s}$, der Güte $Q_{q}$ und der Dicke $l_{q}$ der Quarzwandler. Abweichend zu [88], wo $Q_{q} \approx 10^{3}$ gesetzt wird, ergeben sich für die hier verwendeten Resonatoren sinnvolle Korrekturen für Flüssigkeiten bekannter Dämpfung mit $Q_{q} \approx 1.5 \cdot 10^{4}[140]$.

Wie man an Gleichung (6.14) erkennt, erhält man immer dann signifikante Korrekturen, wenn die Dämpfung zwischen Meß- und Referenzflüssigkeit besonders unterschiedlich ist. Es zeigt sich, daß außerhalb der Quarzresonanzen die Korrektur nach Kononenko nur wenige Prozent ausmacht und damit innerhalb der üblichen Gesamtfehlers liegt. Sie stellt jedoch insgesamt eine systematische Verbesserung dar.

\subsubsection{Bestimmung der Schallgeschwindigkeit}

In Kapitel 3.3 wird eine sehr genaue Methode vorgestellt, mit dessen Hilfe es möglich ist, die Schallgeschwindigkeit gegenüber einer Referenz sehr genau bestimmen zu können. Dabei wird ein Doppelresonator verwandt. Jedoch kann man auch mittels eines Einzelresonators die Schallgeschwindigkeit gut ermitteln, allerdings auf eine andere Weise, im Vergleich zur Methode des Doppelresonators. Bei der Bestimmung der Schallgeschwindigkeit müssen zum einen die Wandlereigenschaft mit hinzugezogen werden, so daß nicht die einfache Beziehung aus (6.4) verwendet werden kann. Wie bereits erläutert, wird nur genau zwischen zwei aufeinanderfolgenden Quarzresonanzen eine $\lambda / 4$-Transformation, ausgehend vom schallweichen Übergang QuarzLuft, ein schallharter Übergang Quarz-Flüssigkeit $(r=1)$ erreicht. Dann stimmt die effektive Länge $l$ aus Gleichung (6.4) des Resonators mit der geometrischen Länge überein. In der Nähe der Wandlerresonanzen ist jedoch $|r|<1$ und geht gegen Null, so daß sich die Schalldruckbäuche zunehmend innerhalb der Wandler befinden. Dieses hat eine scheinbare Vergrößerung der Länge $l$ des Resonators zur Folge und bedingt somit eine Verkleinerung der Frequenzabstände zwischen den Hauptresonanzen. Labhardt [94] gibt eine Formel an, mit der sich unter Berücksichtigung der Wandlereigenschaften und unter Vernachlässigung der Flüssigkeitsdämpfung und des endlichen Zellradius die Schallgeschwindigkeit aus der Lage der Hauptmoden berechnen läßt:

$$
\begin{aligned}
f_{n}-f_{n-1} & =\frac{c_{s}}{2 \pi l} \arccos \left(\frac{\left(g_{n}^{2}-1\right)\left(1-g_{n-1}^{2}\right)-4 g_{n} g_{n-1}}{\left(g_{n}^{2}+1\right)\left(g_{n-1}^{2}+1\right)}\right) \\
\text { mit } \quad g_{j} & =\frac{\rho_{s} c_{s}}{\rho_{q} c_{q} \tan \left(\pi \frac{f_{j}}{f_{q}}\right)} .
\end{aligned}
$$

Für die Berechnung der Schallgeschwindigkeit muß zunächst die Zellänge $l$ bekannt sein. Sie wird im allgemeinen vorher mit einer Flüssigkeit bekannter Schallgeschwindigkeit (Wasser) bestimmt. Dabei errechnet sich die Zellänge als Mittelwert aus allen Differenzen aufeinander folgender Resonanzfrequenzen der gemessenen Hauptmoden, im Gegensatz zur Methode, wie sie beim Doppelresonator verwandt wird, 
bei dem nur eine Resonanz relativ zu einer anderen Resonanz betrachtet wird. Mit dem so berechnetem $l$ kann dann die Schallgeschwindigkeit einer unbekannten Meßflüssigkeit mittels Gleichung (6.15) bestimmt werden. Es hat sich gezeigt, daß sich auch für den plan-konkaven Resonator mit Gleichung (6.15) sinnvolle Ergebnisse erzielen lassen. Die Länge $l$ des Resonator muß allerdings dann als effektive Größe betrachtet werden.

\subsubsection{Meßplatz und Resonatormeßzellen}

\section{Blockschaltbild des Meßplatzes}

In Abb. 6.2 ist der schematische Aufbau des Resonatormeßplatzes dargestellt. Der Ultraschallreso-

nator (1) besteht aus einer zylinderförmigen Zelle, die mit Meßflüssigkeit gefüllt ist. An den Stirnflächen ist die Meßzelle durch zwei piezoelektrische Quarzwandler abgeschlossen. Sie ermöglichen durch ihre beidseitige Goldbedampfung als Sende- (1a) und Empfangswandler (1b) eine elektro-

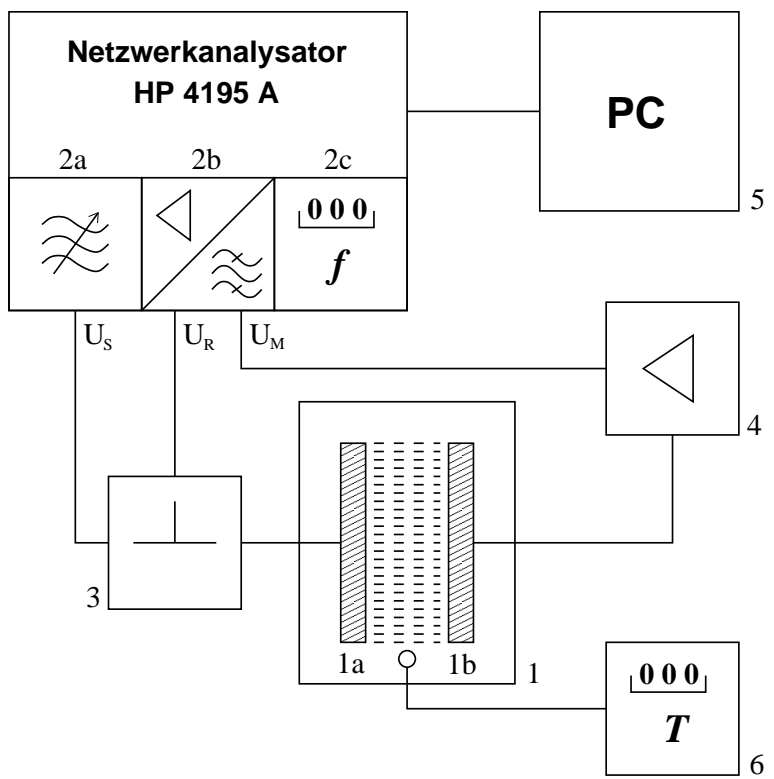

Abb. 6.2: Blockschaltbild des Resonatormeßplatzes: (1) Meßzelle mit (1a) Sende- und (1b) Empfangswandler, (2) Netzwerkanalysator mit (2a) Signalausgang und den Eingängen für (2b) Referenz- und (2c) Empfangssignal, (3) Leistungsteiler, (4) in 10 dB-Schritten steuerbarer 40 dB Verstärker oder FET-Verstärker,

(5) Prozeßrechner, (6) Digitalthermometer

akustische Kopplung. Angeregt durch die sinusförmige Wechselspannung des Netzwerkanalysators (HP 4195 A), führt der Sendequarz aufgrund des reziproken, piezoelektrischen Effektes Dickeschwingungen aus und strahlt so eine Schallwelle gleicher Frequenz in den Innenraum des Resonators ab. Die Schallwelle wird bedingt durch die schallweichen Übergänge an der Grenzfläche Schallwandler-Luft wieder in den Innenraum des Resonators reflektiert und durchläuft die Flüssigkeit so mehrmals. Dadurch bildet sich bei geeigneter Frequenz ein Resonanzsystem mit stehendem Schallfeld aus. Am Empfangswandler wird der dort herrschende Schalldruck wieder in eine zu ihm proportionale Spannung transformiert. Um das Signal-Rausch-Verhältnis zu verbessern, wird dieses Signal in Abhängigkeit der Amplitude verstärkt (4) und anschließend dem Netzwerkanalysatoreingang (2c) zugeführt. Das Sendesignal wird im Leistungsteiler (3) aufgeteilt und liegt als Referenz ebenfalls am Analysator an (2b). Das vom Netzwerkanalysator aufgenommene, normierte, komplexwertige Empfangssignal wird durch die zugehörigen Werte von Pegelabfall und Phasenlage einem Prozeßrechner zur weiteren Bearbeitung 
übermittelt. Neben der Verarbeitung der Meßdaten steuert der Rechner auch die Einstellungen am Analysator während der Messung der einzelnen Resonanzen, wobei er zusätzlich den Verstärkungsgrad des Empfangssignals regelt, um ein Übersteuern des Netzwerkanalysators zu verhindern. Mit Hilfe des Digitalthermometers (6) kann während einer Messung die Temperatur überwacht werden.

\section{Die verwendeten Resonatormeßzellen}

Es werden insgesamt zwei verschiedenen Resonatoren verwendet, deren Konstruktion derart aufeinander abgestimmt ist, daß die Meßfrequenzbereiche aneinander anschließen. Im tieffrequenten Bereich kommt ein sogenannter plan-konkaver Resonator mit $1 \mathrm{MHz}$ Wandlern zum Einsatz, bei dem einer der Wandler eine konkave Geometrie besitzt, um eine Fokussierung der reflektierten Schallwelle zu bewirken. Somit lassen sich störende Beugungsverluste minimieren. Der Resonator ist ausführlich in [25] beschrieben. Im höherfrequenten Bereich wird ein plan-planer Resonator mit $4 \mathrm{MHz}$ Wandlern verwendet, der aufgrund der kleineren Ausmaße des Resonators und der höheren Grundresonanzfrequenz der Wandler im Frequenzbereich oberhalb des $1 \mathrm{MHz}-$ Resonators anschließt. Ausführliche Beschreibungen dieser Meßzelle finden sich in [72] [94]. In Tab. 6.1 sind die technischen Daten beider Resonatoren aufgeführt.

\begin{tabular}{|c|ccc|cccc|c|}
\hline Bezeichnung & $\begin{array}{c}f_{q} \\
{[\mathrm{MHz}]}\end{array}$ & $\begin{array}{c}K \\
{[\mathrm{~m}]}\end{array}$ & $\begin{array}{c}r_{q} \\
{[\mathrm{~mm}]}\end{array}$ & $\begin{array}{c}l \\
{[\mathrm{~mm}]}\end{array}$ & $\begin{array}{c}R \\
{[\mathrm{~mm}]}\end{array}$ & $\begin{array}{c}V \\
{[\mathrm{ml}]}\end{array}$ & $\begin{array}{c}f_{g} \\
{[\mathrm{kHz}]}\end{array}$ & $\begin{array}{c}\text { Meßbereich } \\
{[\mathrm{MHz}]}\end{array}$ \\
\hline \hline plan-konkav & 1 & $\infty / 2.0$ & 40 & $\sim 19$ & 35 & $\sim 75$ & $\sim 40$ & $0.2 \ldots 2.8$ \\
plan-plan & 4 & $\infty / \infty$ & 10 & $\sim 6$ & 8.4 & $\sim 1.3$ & $\sim 125$ & $1 \ldots 15$ \\
\hline
\end{tabular}

Tabelle 6.1: Resonatordaten: $f_{q}$ : Grundresonanzfrequenz der Quarzdickenschwingung, $K$ : Krümmungsradius und $r_{q}$ : Radius der Quarzwandler; $l$ : Länge der Flüssigkeitsschicht auf der Mittelachse, $R$ : Radius, $V$ : Volumen und $f_{g}$ : Grundresonanzfrequenz der Zelle.

\subsubsection{Ablauf der Messung}

Die Meßzelle wird zunächst mit bidestilliertem, deionisiertem und entgastem Wasser befüllt und auf die gewünschte Temperatur gebracht. Um eine möglichst gute Benetzung der Wandler zu erreichen und die Bildung von Luftblasen zu verhindern, wird die Flüssigkeit entsprechend langsam in die Meßzelle eingefüllt. Ist dieses geschehen, werden die Wandler mit Hilfe von an den Wandlerhalterung angebrachten Differentialschrauben parallel justiert. Kriterium für eine gute Paralleljustierung ist dabei ein möglichst hoher Pegel und eine kleine Halbwertsbreite bei einer ausgewählten Resonanz, die während der Justierung auf dem Netzwerkanalysator beobachtet werden kann. Beim tieffrequenten plan-konkaven Resonator ist diese Prozedur aufgrund der hohen Fokussierung der Wandler jedoch nicht nötig. Außerdem wird die Temperatureinstellung der Flüssigkeit abgewartet. Da die Schallgeschwindigkeit empfindlich temperaturabhängig ist, können Temperaturänderungen in der Verschiebung der Resonanzfrequenzen $f_{n}$ beobachtet werden. Eine minimale Frequenzdrift bei einer ausgewählten Resonanz mit hoher Güte wird abhängig vom Flüssigkeitsvolumen 
und der Temperaturdifferenz zur Anfangstemperatur nach ca. zwei bis vier Stunden erreicht.

Ist die gewünschte Temperatur erreicht, beginnt die eigentliche Meßprozedur. Der Prozeßrechner bestimmt zunächst grob die Resonanzfrequenz einer Hauptmode über den Maximalwert des Pegels. Anschließend wird die komplexwertige Übertragungsfunktion üblicherweise im Frequenzintervall von der dreifachen, über den 3-dB-Abfall bestimmten Halbwertsbreite um die Resonanzfrequenz an ca. 100 Meßpunkten aufgenommen. An diese so ermittelte Übertragungsfunktion wird zur genauen Ermittlung der Resonanzfrequenz eine Lorentzkurve angepaßt. Danach wird mit der nächst höheren Hauptmode unter Verwendung des vorher ermittelten Frequenzabstandes zwischen den einzelnen Hauptmoden analog verfahren. Diese Meßprozedur wird bis zu einer zuvor festgelegen oberen Frequenzgrenze fortgeführt, wobei jedoch die Bereiche um die Wandlerresonanzen, also $f_{q n} \pm 0.1 \cdot f_{q 1}$ mit $(\mathrm{n}=1,2,3 \ldots)$, ausgelassen werden.

Ist die Wassermessung beendet, wird das Wasser abgelassen und die Meßzelle mit Methanol gespült und mit Stickstoff getrocknet. Die anschließende Befüllung der Zelle mit einer Lipidsuspension gestaltet sich, sofern die Lösung entsprechend gut entgast ist, nicht wesentlich schwieriger als die Wasserbefüllung. Bei genügend langsamer Befüllung kann die Blasenbildung, zu der die Lipidsuspensionen sehr stark neigen, völlig vermieden werden. Somit kann eine sehr gute Benetzung der Wandler erreicht werden. Da die Schallgeschwindigkeit der Lipidsuspensionen fast der von Wasser entspricht, kann die schon erfolgte Wassermessung einerseits zur Bestimmung der Zellänge (Gleichung 6.15), andererseits als Referenz herangezogen werden (Gleichung 6.8). Auf eine weitere Messung einer gesonderten Referenzflüssigkeit wird somit verzichtet.

\subsubsection{Fehlerbetrachtungen}

\section{Temperaturschwankungen}

Aufgrund der Temperaturabhängigkeit der Zellänge und der Schallgeschwindigkeit der Meßflüssigkeit verursacht die endliche Temperaturstabilität auch noch nach angemessener Wartezeit eine Drift der Resonanzfrequenzen der Hauptmoden. Dieses führt zu einer meßbedingten Veränderung der gemessenen Halbwertsbreite der Resonanzkurven, so daß der daraus resultierende Fehler insbesondere beim plan-konkaven Resonator im tieffrequenten Bereich bis zu $30 \%$ betragen kann. Ab ca. $600 \mathrm{kHz}$ aufwärts spielt die Frequenzdrift zunehmend kaum noch eine Rolle und der dadurch verursachte Fehler kann mit ca. $1 \%$ angegeben werden. Beim plan-planen Resonator beträgt die maximale Frequenzdrift zu Beginn einer Messung etwa $0.01 \mathrm{~Hz} / \mathrm{s}$, was sich bei einer Meßzeit einer Hauptmode von ca. 10 Sekunden in einem Fehler von weniger als $1 \%$ niederschlägt.

Während der Messung schwankte die Temperatur maximal um $\pm 0.02^{\circ} \mathrm{C}$. Der aus der Unsicherheit des Absolutwertes der Temperatur resultierende Fehler kann aus der Temperaturabhängigkeit von Dämpfungswerten der gemessenen Systeme mit weniger als $0.1 \%$ abgeschätzt werden und ist somit für den Resonatorbereich vernachlässigbar. 


\section{Fehler des Referenzverfahrens}

Bei relativ tiefen Frequenzen, unterhalb von 500 kHz für den plan-konkaven Resonator bzw. unterhalb von $2 \mathrm{MHz}$ für den plan-planen Resonator, liegen die apparativen Verluste im Bereich derer, wie sie durch schwach dämpfende Flüssigkeiten hervorgerufen werden, und nehmen mit abnehmender Frequenz stark zu (Kap. 6.1.1). Diees führt insbesondere bei niedrigen Frequenzen zu erheblichen systematischen Fehlern, sollten Schallgeschwindigkeit und Dichte zwischen Meß- und Referenzflüssigkeit zu stark voneinander abweichen. Weiter können Fehler entstehen, wenn eine unvollständige Benetzung der Wandler vorliegt. Dies kann selbst bei sehr vorsichtiger Befüllung der Resonatoren hin und wieder einmal vorkommen. Diese hier beschriebenen Fehler sind jedoch aufgrund ihres charakteristischen Frequenzverlaufs als solche $\mathrm{zu}$ erkennen, und werden nicht in der Auswertung berücksichtigt. Im oberen Frequenzbereich ergeben sich die Fehler hauptsächlich durch eine ungenaue Vorgabe der Dämpfungswerte der Referenz. Da die Dämpfungswerte von Wasser jedoch gut bekannt sind, ist der hierdurch hervorgerufene Fehler mit nur ca. $1 \%$ anzugeben.

\section{Fehlerhafte Anpassung des Dämpfungsexponenten}

Die Fehler, welche durch eine fehlerhafte Anpassung der theoretischen Übertragungsfunktion an die gemessenen Werte (Abb. 6.2) resultierwn, können bei dem $1 \mathrm{MHz}$ Resonator und bei dem $4 \mathrm{MHz}$ Resonator bis $6 \mathrm{MHz}$ auf unter $1 \%$ abgeschätzt werden. Über $6 \mathrm{MHz}$ kommt es zunehmend zu einer Beeinflussung der Hauptmode durch Nebenmoden, insbesondere wenn man Messungen genau an der kritischen Umwandlungstemperatur der Lipidsuspension durchführt. Die somit bedingte Unsicherheit in der Bestimmung der Resonanzfrequenzen und der zugehörigen Halbwertsbreiten begrenzt den Meßbereich des $4 \mathrm{MHz}$ Resonator nach oben hin.

\section{Fehler in der Schallgeschwindigkeit}

Der aufgrund von Temperaturschwankungen und Unsicherheiten in der Frequenzlage der Hauptmoden resultierende Fehler bei der Bestimmung der Schallgeschwindigkeit kann mit 0,1 \% angegeben werden.

\section{Resultierender Gesamtfehler}

Berücksichtigt man alle oben aufgeführten Effekte, die zu Fehlern des mit dem Resonatorverfahren ermittelten Dämpfungsexponenten führen, so ergeben sich die in Tabelle 6.2 aufgeführten relativen Fehler:

\subsection{Das Schwingungspulstransmissionsverfahren}

Da der Frequenzbereich der Resonatoren zu hohen Frequenzen hin begrenzt ist, kam, wie zuvor dargelegt, oberhalb von $15 \mathrm{MHz}$ ein anderes Meßverfahren zum Einsatz: das Schwingungspulstransmissionsverfahren. Ausführliche Beschreibungen dieses Meßverfahrens und der verwendeten Meßzellen findet sich in [125] [103] . 


\begin{tabular}{|c|c|c|c|}
\hline $\begin{array}{c}f_{q} \\
{[\mathrm{MHz}]}\end{array}$ & $\begin{array}{c}\text { Meßintervall } \\
{[\mathrm{MHz}]}\end{array}$ & $\begin{array}{c}\Delta \alpha / \alpha \\
\alpha / f^{2}<5 \cdot 10^{-14}\left[\mathrm{~m} / \mathrm{s}^{2}\right]\end{array}$ & $\begin{array}{c}\Delta \alpha / \alpha \\
\alpha\end{array}$ \\
\hline \hline \multirow{3}{*}{1} & $0.2-0.9$ & $10 \%$ & $5 \cdot 10^{-14}\left[\mathrm{~m} / \mathrm{s}^{2}\right]$ \\
& $1.1-1.8$ & $5 \%$ & $3 \%$ \\
& $2.3-2.8$ & $6 \%$ & $4 \%$ \\
\hline \multirow{4}{*}{4} & $1.0-3.6$ & $10 \%$ & $5 \%$ \\
& $4.4-7.6$ & $5 \%$ & $3 \%$ \\
& $8.4-15$ & $6 \%$ & $6 \%$ \\
\hline
\end{tabular}

Tabelle 6.2: Relative Gesamtfehler des Dämpfungsexponenten für das Resonatorverfahren, unterteilt nach Frequenzbereich und Dämpfung pro Frequenzquadrat $\alpha / f^{2}$.

\subsubsection{Meßprinzip}

Ein hochfrequentes, harmonisches, elektrisches Signal wird pulsförmig moduliert (minimale Pulsdauer $\tau_{\text {min }} \approx 1.5 \mu \mathrm{s}$ ) und dann auf einen piezoelektrischen Schallwandler gegeben. Dieser strahlt einen Schallpuls gleicher Frequenz in die Flüssigkeit ab, und nachdem die Schallwelle die Flüssigkeit durchlaufen hat, wird dieser wieder durch einen in Schallfeldrichtung beweglichen Schallwandler in einen elektrischen Schwingungspuls umgewandelt. Prinzipiell liegt somit ein sehr resonatorähnlicher Aufbau vor. Zwei piezoelektrische Wandler sind in einigem Abstand x parallel zueinander angebracht und zwischen ihnen befindet sich die zu messende Flüssigkeit. Bei einer kontinuierlichen Anregung mit der Spannung $U_{a}$ ergibt sich also das Empfangssignal und die Schalldruckamplitude $p_{e}$ aus der Überlagerung der zwischen den Wandlern hin- und herreflektierten Schallwellen. Unter Annahme einer ebenen Wellenausbreitung erhält man analog zur Übertragungsfunktion bei den Resonatoren:

$$
p_{e} \sim\left|\frac{e^{-\gamma x}}{1-r^{2} e^{2 \gamma x}}+A_{u} e^{i \phi_{u}}\right|
$$

Hierbei sind $A_{u}$ die relative Amplitude und $\phi_{u}$ die Phase des Übersprechens und $\gamma=\alpha+i 2 \pi f / c_{s}$.

\section{Vernachlässigung der Mehrfachreflexion}

Der Term $r^{2} e^{2 \gamma x}$ beschreibt die Auswirkungen der Mehrfachreflexionen. Sollte jedoch die Dämpfung der Flüssigkeit entsprechend hoch und der Abstand der beiden Wandler groß genug $(\alpha>3 / x)$ sein, so ist die Welligkeit mit der Periodenlänge $\lambda / 2$ vernachlässigbar. Es ergibt sich dann mit $\left|r^{2} e^{2 \gamma x}\right| \ll 1$ und Gleichung (6.16) ein rein exponentielles Abfallen der Schalldruckampitude am Empfangswandler in Abhängigkeit des Abstandes beider Wandler:

$$
p_{e} \sim e^{-\alpha x}
$$

Beide Wandler werden nun ausgehend von einer Startposition kontinuierlich auseinandergefahren und dabei wird die am Empfangswandler ankommende Schalldruckamplitude aufgenommen, und so der Dämpfungsexponent über den exponentiellen Abfall des Schalldruckes direkt bestimmt. Durch das Pulsen des kontinuierlichen 
Signals wird das zeitliche Trennen des elektrischen Übersprechens $A_{u} e^{i \phi_{u}}$ vom am Empfangswandler anliegenden realen akustischen Signal ermöglicht. Um diese Trennung auch bei vergleichsweise hohen Frequenzen zu gewährleisten, wo die Laufzeit des Pulses möglicherweise kürzer als die minimale Pulslänge sein kann, werden Verzögerungsleitungen aus Saphir oder Quarz verwendet. Durch das Pulsen des Signals werden auch Mehrfachreflexionen vermieden, sollte der Abstand $x$ größer als $\tau c_{s} / 2$ sein ( $\tau$ ist die Pulsdauer).

\section{Welligkeit bei geringem Abstand der Wandler}

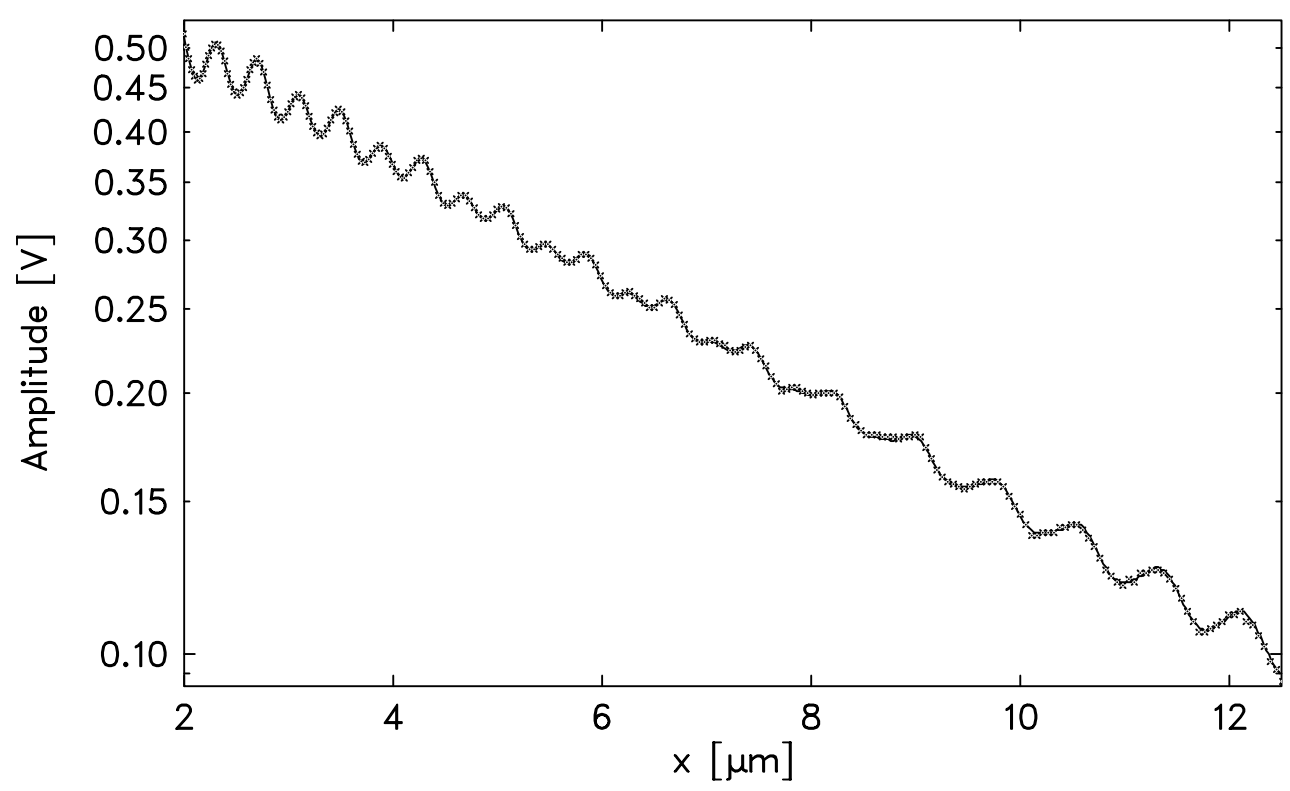

Abb. 6.3: Gemessene Spannungsamplitude $(x)$ über dem Abstand $x$ bei einer Frequenz von $1 \mathrm{GHz}$ der PZT-Zelle bei $25^{\circ} \mathrm{C}$, durchgezogene Kurve: Graph der Funktion nach Gleichung (6.16) mit aus der Anpaßrechnung gewonnenen Parameterwerten.

Es tritt eine merkliche Welligkeit in der gemessenen Übertragungsfunktion auf, sollte durch die endlich Pulslänge der Abstand der Wandler $x$ kleiner als $\tau c_{s} / 2$ sein, ein ungünstiges Signal-Rausch-Verhältnis herrschen oder die maximal mögliche Meßstrecke einen sehr kleinen Startabstand erfordern. Dies ist grundsätzlich bei der hochfrequenten PZT-Apparatur der Fall (siehe Kapitel 6.2.3), wo der maximale Hub des Piezotranslators auf $40 \mu \mathrm{m}$ beschränkt bleibt. Somit ist hier eine Vernachlässigung der Welligkeit wie bei allen anderen Pulsmeßzellen nicht möglich. Um die Schalldämpfung und die Schallgeschwindigkeit zu bestimmen, wird an die gemessenen Werte die Übertragungsfunktion jeweils nach jeder erfolgten Messung (Gleichung (6.16)) angepaßt.

Das elektrische Übersprechen spielt bei der PZT-Meßzelle erst bei Frequenzen oberhalb von $2 \mathrm{GHz}$ eine merkliche Rolle und wird somit bei den Anpaßrechnungen nicht berücksichtigt. Abb. 6.3 zeigt eine solche typische Meßkurve der PZT-Meßzelle bei ca. $1 \mathrm{GHz}$ mit angepaßter Übertragungsfunktion. 


\subsubsection{Meßplatz der Pulstransmissionszellen}

Abb. 6.4 zeigt den prinzipiellen Aufbau des Meßplatzes für die Pulszellen. Die durchgezogene Linie zeigt den Weg des Signals durch den Meßzweig, während die gestrichelte Linie den Weg durch den Referenzzweig darstellt. Das Signal des Synthetisiersenders (1) wird mit dem Signal des Rechteck-Pulsgenerators (3) mit Hilfe eines PIN-Diodenschalters (2) entsprechend moduliert und so ein kurzer Schwingungspuls mit der am Synthetisiersender eingestellten Frequenz erzeugt. Danach wird das Signal um maximal $40 \mathrm{~dB}$ verstärkt (4) und anschließend auf ein Koaxialrelais (5) gegeben, welches das Signal entweder durch den Meßzweig mit der Meßzelle (7) (rechts) oder durch den Referenzzweig mit einem Cut-Off-Dämpfungsglied (8) (links) leitet. In der Meßzelle liegt das pulsmodulierte Signal am Sendewandler (7a) an, der durch die elektrische Anregung ein entsprechendes akustisches Signal in die Flüssigkeit abstrahlt. Der Puls durchläuft die Flüssigkeit und trifft auf den gegenüber dem Sendewandler befindlichen Empfangswandler (7b), der den akustischen Puls wieder in ein elektrisches Signal zurückwandelt. Die Bauteile (6) lassen eine manuelle Amplitudenregelung im Meßzweig zu. Bei kleinen Frequenzen und damit geringerer Dämpfung ermöglicht das Dämpfungsglied vor der Meßzelle eine optimale Abstimmung zwischen Amplitude im Meß- und Referenzzweig, während bei hohen Frequenzen durch eine Impedanzanpassung mit Kurzschlußstrichleitung das Signal-Rausch-Verhältnis hinter dem Empfangswandler erheblich verbessert wird.

Das zweite Koaxialrelais (5) führt nun das durch die Meßzelle bzw. durch das CutOff-Glied gelaufene Signal dem Empfangsteil zu. Das Signal wird mit Hilfe eines weiteren Oszillators (11) gemischt (10) und durchläuft anschließend einen Filter (12), der auf die gewünschte Mischfrequenz eingestellt ist. Das so erhaltene Signal wird nochmals in (13) variabel verstärkt und sowohl zu Kontrollzwecken auf einen Oszillographen (15) gegeben, als auch mittels einer Sample-and-Hold-Schaltung und einer A/D-Karte (14) zur weiteren Auswertung einem Prozeßrechner (22) zugeführt. Der Prozeßrechner übernimmt neben der reinen Auswertung der Daten vielfältige Aufgaben bei der Steuerung des gesamten Aufbaus. Der Rechner steuert mit Hilfe eines Pulsgenerators (16) das Triggersignal für den Oszillographen und die Sampleand-Hold-Schaltung. Das Startsignal wird an den Pulsgenerator (3) gegeben und die Koaxialrelais (6) werden über eine spezielle Treiberkarte mit Hilfe des Rechners geschaltet. Die Einstellung des Abstandes und das während der Messungen nötige gleichmäßige Auseinanderfahren der Wandler (7a und 7b) wird über eine Motorsteuerungseinheit (17) und einem Schrittmotor (18) erreicht. Lediglich bei der hochfrequenten PZT-Apparatur werden keine Schrittmotoren verwendet. Dort gibt der Rechner über einen A/D-Wandler eine Spannung zwischen 0 und 10 Volt an einen Hochspannungverstärker (bis $1 \mathrm{kV}$ ), dessen Hochspannung an einem PiezoTranslator (PI, Waldbronn) anliegt. Durch die Variation dieser Hochspannung werden die für die Pulsmessung nötigen Abstandsänderungen von maximal $40 \mu \mathrm{m}$ mit einer Reproduzierbarkeit von $5 \mathrm{~nm}$ erreicht. Die Messung des Abstandes (19) erfolgt üblicherweise über einen optischen Längentaster (Heidenhain MT60, MT25, MT10). Nur bei der PZT-Meßzelle wird ein induktiver Längentaster verwendet (LVDT, Schlumberger), welcher vor der Messung mittels eines optischen Längentasters (Heidenhain MT12) kalibriert wird. Im Referenzzweig wird die Stellung des Cut-Off-Dämpfungsgliedes über die gefahrene Schrittzahl des Motors ermittelt. Mit Hilfe eines Digitalthermometers (20) wird die Temperatur in der Meßzelle überwacht. 


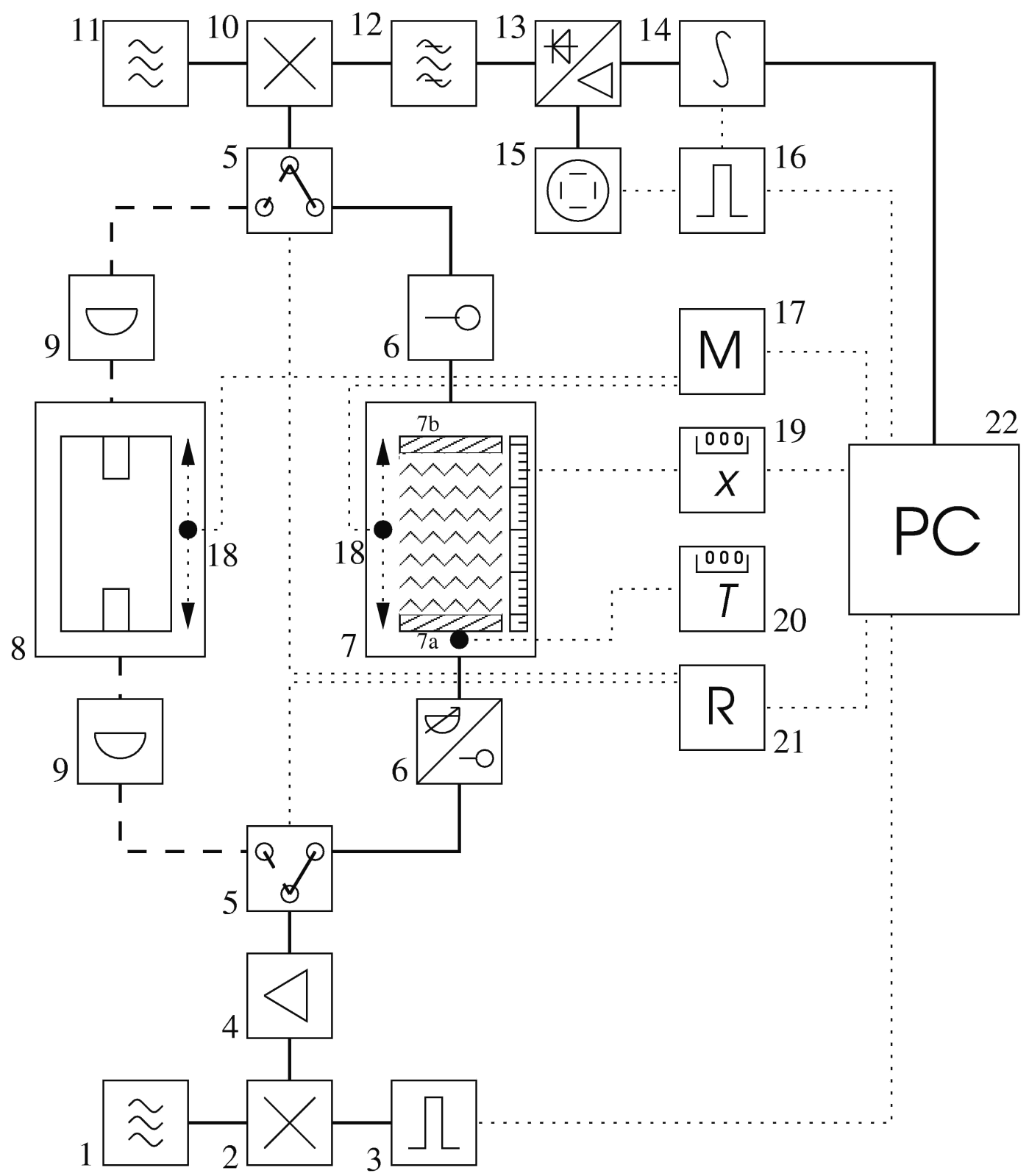

Abb. 6.4: Blockschaltbild des Pulstransmissionsmeßplatzes (durchgezogene Linie: Signalleitung durch den Meßzweig, gestrichelte Linie: Signalweg durch den Referenzzweig, gepunktete Linien: Steuerleitungen): (1) Synthetisiersender; (2) Modulator; (3) Pulsgenerator; (4) Verstärker; (5) Koaxialrelais; (6) Amplitudenregulierung (variables Dämpfungsglied, Kurzschlußstichleitungen); (7) Meßzelle mit Sende-- (7a) und Empfangswandler (7b); (8) Cut-Off-Dämpfungsglied; (9) 10-dB Dämpfungsglied als Reflexionsschutz; (10-13) Überlagerungsempfänger; (14) Sampleand-Hold-Schaltung und A/D-Wandler; (15) Oszillograph; (16) Pulsgenerator; (17) Motorsteuerung oder D/A-Wandler mit 1 kV-Verstärker; (18) Schrittmotor oder Piezo-Translator; (19) optischer oder induktiver Taster zur Abstandsmessung; (20) Digitalthermometer; (21) Relaistreiberkarte; (22) Prozeßrechner. 


\subsubsection{Die Pulsmeßzellen}

Um den Frequenzbereich oberhalb der Resonatoren von $15 \mathrm{MHz}$ bis 4,6 GHz abzudecken, werden insgesamt vier verschiedene Pulsmeßzellen verwendet, deren Meßbereiche jeweils aneinander anschließen. Dabei verdient die Piezotranslatormeßzelle besondere Aufmerksamkeit, da sie technisch eine Sonderrolle einnimmt und in diesem Frequenzbereich höchste technische Anforderungen an die Abstandsmessung der Wandler gestellt werden.

\section{Die 1-MHz- und $10 \mathrm{MHz}-P$ ulszelle}

Beide Meßzellen decken zusammen den Frequenzbereich von ca. $15 \mathrm{MHz}$ bis max. $430 \mathrm{MHz}$ ab und sind in etwa baugleich. Als Schallwandler kommen Lithiumniobat$\left(\mathrm{LiNbO}_{3}\right)$-Wandler bei der $10 \mathrm{MHz}-\mathrm{Pulszelle}$ und Quarzwandler bei der $1 \mathrm{MHz}-$ Pulszelle zum Einsatz. Eine genauere Konstruktionsbeschreibung findet sich in [125] [77] für die $10 \mathrm{MHz}-Z$ elle und in [82] für die $1 \mathrm{MHz}-Z$ elle. Die möglichen Meßfrequenzen ergeben sich entsprechend durch die jeweiligen Resonanzfrequenzen der Wandler und deren ungeradzahliger Vielfache. Nach oben hin wird der meßbare Frequenzbereich durch das mit höherer Ordnung der Oberschwingung abnehmende Signal-Rauschverhältnis bestimmt. Die untere Grenze des Frequenzbereiches ist durch endliche Länge des Meßweges durch die Flüssigkeit bestimmt, da bei relativ geringer Dämpfung der Flüssigkeit durch einen zu kurzen Meßweg nur ein unzureichender Abfall der Schalldruckamplitude zu verzeichnen ist.

\section{Die Hyperschallpulszelle}

Bei dieser Meßzelle, die den Frequenzbereich von ca. $500 \mathrm{MHz}$ bis $1 \mathrm{GHz}$ abdeckt, wird ein anderes Verfahren zu Schallerzeugung benutzt [81]. Ein etwa $10 \mathrm{~mm}$ langer piezoelektrischer $\mathrm{LiNbO}_{3}$-Stab mit einem Durchmesser von $3 \mathrm{~mm}$ wird nach dem Prinzip der Oberflächenanregung nach Bömmel und Dransfeld [14] angeregt. Die Schalldetektion geschieht reziprok analog. Beide Wandler ragen ca. $1 \mathrm{~mm}$ aus ihrer Ummantelung in den Meßbereich, und auf der anderen Seite ebenfalls ca. 1 $\mathrm{mm}$ in einen Koaxialresonator, der durch Verschieben eines Kurzschlußschiebers auf die jeweilige Frequenz abgestimmt werden kann. Das Ein- und Auskoppeln des elektromagnetischen Feldes geschieht über Induktionsschleifen. Somit können die Meßfrequenzen in einem weiten Bereich beliebig ausgewählt werden. Dabei wird die Frequenzuntergrenze durch die Mindestlänge des Kurzschlußschiebers von $\lambda / 4$ auf $500 \mathrm{MHz}$ begrenzt. Die obere Grenze des meßbaren Frequenzbereiches wird durch das zunehmende schlechte Signal-Rausch-Verhältnis gebildet. Der derzeitige Zustand der Apparatur ließ es zu, bis ca. 1,1 GHz Meßpunkte aufzunehmen.

Die Abstandsmessung bei dieser Apparatur erfolgt wie bei den anderen Pulszellen mittels eines optischen Längentasters. Aus Konstruktionsgründen kann diese Längenmessung jedoch nicht auf der Achse vorgenommen werden, auf der sich die Schallwandler befinden. Die Messung erfolgt ca. $5 \mathrm{~cm}$ von dieser Achse versetzt auf der scheibenförmigen Wandlerhalterung. Somit ist eine prinzipielle Ungenauigkeit, die auf ein Verkippen dieser Platte zurückzuführen ist, nicht auszuschließen. Durch die Verwendung von spielfreien Kugellagern wird versucht diesen Fehler möglichst gering zu halten. Eine genaue Beschreibung der Apparatur findet sich in [103]. 


\section{Die Piezotranslatorpulszelle}

Da sich oberhalb von ca. $1 \mathrm{GHz}$ die benötigten Meßstrecken derart verkürzen, daß eine verläßliche Positionierung mittels Schrittmotoren nicht mehr möglich ist, muß für den Frequenzbereich oberhalb von $1 \mathrm{GHz}$ eine andere Methode gefunden werden [81]. Die Änderung des Abstandes der Wandler wird mittels eines Piezotranslators bewerkstelligt. An diesen wird eine frei einzustellende Hochspannung von 0 bis 1000 Volt angelegt, was dazu führt, daß der Piezotranslator einen maximalen Hub von ca. $40 \mu m$ erzeugt, welches für den Meßbereich ab $1 \mathrm{GHz}$ völlig ausreichend ist. Das Wandlersystem bei dieser Apparatur wird von einem piezoelektrischen ZnO-Film gebildet, der auf eine Saphir- $\left(\mathrm{Al}_{2} \mathrm{O}_{3}\right)$-Verzögerungsleitung (mit einer Länge von 10 $\mathrm{mm}$ und einem Durchmesser von $7 \mathrm{~mm}$ ) auf eine Fläche von ca. $12 \mathrm{~mm}^{2}$ gesputtert wird [81]. Die so erhaltene, ca. $1 \mu m$ starke ZnO-Schicht wird wie bei der 10 MHz-Pulszelle zu Dickeschwingungen angeregt, wobei eine etwa $0,1 \mu m$ dicke Goldund Chromschicht als Grundelektrode dient. Das gesamte System, bestehend aus der ZnO-Schicht, der Goldelektrode und der Saphir-Verzögerungleitung, bildet ein Resonanzsystem, welches durch die verschieden Impedanzen zwischen den diversen Materialien und durch deren Kopplung die breitbandige Grundresonanz der $\mathrm{ZnO}-$

Schicht bei ca. $f_{0} \approx 3 G H z$ verschiebt und verformt. Gute Übertragungseigenschaften werden somit empirisch bei den Frequenzen 0.8 - 2.6 GHz (mit einem Maximum bei ca. $1.3 \mathrm{GHz}$ ) und $3.7-4.6 \mathrm{GHz}$ (mit einem Maximum bei $3.9 \mathrm{GHz}$ ) gefunden. Die Verwendung von Saphir in der Verzögerungsleitung hat im Gegensatz zu Quarzglas $\left(\alpha / f^{2}=0.13 \mathrm{~s}^{2} / \mathrm{m}\right)$ bei der $10 \mathrm{MHz}-$ Pulszelle den Vorteil einer sehr geringer Schalldämpfung $\left(\alpha / f^{2}=0.0021 \mathrm{~s}^{2} / \mathrm{m}\right)$. Jedoch führt die Verwendung von Saphir auch zu einer besonders hohen Impedanz an der Grenzfläche zwischen Saphir und der Flüssigkeit. Es hat sich jedoch gezeigt, daß die Genauigkeit der Apparatur auch ohne eine spezielle Impedanzanpassung ausreichend hoch ist.

\section{Zusammenfassung}

Die technischen Daten aller oben beschriebenen Pulszellen sind in Tabelle 6.3 zusammengefaßt.

\subsubsection{Ablauf der Messung}

Zunächst wird das Meßvolumen der Meßzelle mit der zu messenden Flüssigkeit, die zuvor entgast wurde, vorsichtig befüllt. Ein Deckel schützt vor Konzentrationsänderungen während der Messung. Bei der PZT-Apparatur wird das Meßvolumen mit Silikonringen entsprechend abgedichtet.

Vor Beginn jeder Messung wird die Pulstransmissionszelle auf die gewünschte Temperatur gebracht, welche durch das Digitaltermometer gemessen wird. Im Fall der PZT-Apparatur wird schon vor der Befüllung mit der Meßflüssigkeit eine Kalibrierung des induktiven Längentasters vorgenommen.

Die gewünschte Frequenz wird am Sender eingestellt und der Überlagerungsempfänger auf das Signal des Cut-Off-Dämpfungsgliedes abgestimmt. Dieses geschieht während der Beobachtung des Pulses auf dem Oszilloskop. Danach wird in den Meßzweig geschaltet und die Amplitude des Pulses wird nach dem Wählen eines Startabstandes der Wandler mittels des variablen Dämpfungsgliedes bzw. den Stichleitungen 


\begin{tabular}{|l|c|c|c|c|}
\hline Bezeichnung & $\begin{array}{c}1-\mathrm{MHz}^{-} \\
\text {Pulszelle }\end{array}$ & $\begin{array}{c}10-\mathrm{MHz}^{-} \\
\text {Pulszelle }\end{array}$ & $\begin{array}{c}\text { Hyperschall- } \\
\text { zelle }\end{array}$ & $\begin{array}{c}\text { PZT- } \\
\text { Zelle }\end{array}$ \\
\hline \hline Wandlermat. & Quarz & LiNbO $_{3}$ & LiNbO & ZnO \\
$r_{q}[\mathrm{~mm}]$ & $20 / 30$ & 6 & 1.5 & 3.5 (V.-leitg.) \\
$f_{q}[\mathrm{MHz}]$ & 1.00 & 10.8 & breitbandig & $\approx 1300$ \\
$f_{n}$ & $(2 n+1) f_{q}$ & $(2 n+1) f_{q}$ & $0.5-2.0 \mathrm{GHz}$ & $1.1-2.6 \mathrm{GHz}$ \\
$f_{\max }[\mathrm{MHz}]$ & 63 & 435 & 2000 & 4600 \\
$\tau[\mu \mathrm{s}]$ & $10-5$ & $4-2$ & 1.5 & 1.5 \\
Taster & optisch, MT60 & optisch, MT25 & optisch, MT10 & induktiv, LVDT \\
Tasterposition & axial & axial & 5 cm achsenparallel & axial \\
$x_{\min }[\mathrm{nm}]$ & 125 & 125 & 0.5 & 8 \\
$x_{\max }[\mathrm{mm}]$ & 40 & 25 & 1 & 0.04 \\
$V[\mathrm{ml}]$ & $\approx 130$ & $\approx 10$ & $\approx 3$ & $\approx 0.5$ \\
\hline
\end{tabular}

Tabelle 6.3: Technische Daten der Meßzellen mit Pulstransmissionsverfahren: Radius $r_{q}$ und Grundresonanzfrequenz $f_{q}$ der Kristallwandler; $f_{n}$ : mögliche Meßfrequenzen und $f_{\max }$ : maximale Meßfrequenz; $\tau$ : Pulslänge; $x_{\min }$ : minimale und $x_{\max }$ : maximale Abstandsänderung zwischen den Wandlern; $V$ : Flüssigkeitsvolumen der Meßzelle.

so eingestellt, daß sich der Pegel der Sample-And-Hold-Schaltung im Linearitätsbereich unterhalb von $800 \mathrm{mV}$ befindet.

Mit Hilfe von Justierschrauben kann nun die Parallelität der Wandler nachgeregelt werden. Dieses ist bei der $10 \mathrm{MHz}$-Pulszelle bei jeder neu eingedellten Frequenz nötig, während es bei der Hyperschallzelle und bei der PZT-Zelle nur einmalig erfolgen muß.

Nun wird eine Meßstrecke entsprechend eines Pegelabfalls von 3 - 15 dB gewählt. Die Messung wird gestartet und die Apparatur fährt die Wandler kontinuierlich auseinander. Dabei werden an ca. 400 Stützstellen jeweils der Abstand der Wandler und der zugehörige Amplitudenwert der Samle-And-Hold-Schaltung aufgenommen. Danach wird wieder in den Referenzzweig geschaltet und ca. 60 Spannungswerte mit Hilfe des Cut-Off-Gliedes, die allerdings innerhalb des gemessenen Spannungsintervalles aus dem Meßzweig liegen, aufgenommen. An diese Werte werden zur Glättung Polynomabschnitte angepaßt und so die Kennlinie der gesamten Elektronik bestimmt. So wird mittels der Daten aus dem Referenzzweig und der Werte aus dem Meßzweig der Dämpfungswert $\alpha$ bei der oben eingestellten Frequenz errechnet. Anschließend kann eine neue Meßfrequenz eingestellt werden.

\subsubsection{Meßfehler}

Die zumeist apparativ bedingten Fehler sind bereits mehrfach beschrieben worden [125], so daß in dieser Stelle nicht detailliert darauf eingegangen werden soll. Jedoch ergaben sich Fehler, die nur spezifisch mit den gemessenen Lipidsuspensionen zusammenhängen.

Die $1 \mathrm{MHz}$-Pulszelle und die $10 \mathrm{MHz}-\mathrm{Pulszelle} \mathrm{zeigten} \mathrm{im} \mathrm{verwendeten} \mathrm{Temperatur-}$ und Meßbereich keine besonderen Abnormitäten, so daß die üblichen apparativen Fehler anzugeben sind.

Die Thermostatierung der Hyperschallapparatur $(500 \mathrm{MHz}-1,1 \mathrm{GHz})$ muß insbe- 
sondere bei Temperaturen, die sich nicht in der Nähe der Raumtemperatur befinden, als nicht zufriedenstellend anesehen werden. Das Meßvolumen ist lediglich durch das wandlerhaltende Metall mit den Wasser durchflossenen Bereichen der Zelle verbunden. Die Wärmeleitfähigkeit des Metalls sorgt für die gewünschte Thermostatierung. Jedoch ist dieser Bereich der Apparatur nur durch eine Plexiglas-Wand von den Außenwelt und damit von der Raumtemperatur getrennt. Versuche die gesamte Meßzelle mit Isoliermaterial einzudecken, brachten nur geringfügige Verbesserungen. Somit kann die Temperaturkonstanz nicht immer über den Zeitraum einer Messung gewährleistet werden. Der dadurch entstandene Fehler in $\alpha$ wird bei Temperaturen $<15^{\circ} \mathrm{C}$ und $>35^{\circ} \mathrm{C}$ mit $3 \%$ abgeschätzt.

Zusätzlich dazu kommt das schon angesprochene nicht genau einzuschätzende Verkippen der Platte, auf der sich die Wandlerhalterung, die Abstandsmessung und die Spindel befindet. Eine merkliche Zunahme des Fehlers bei hohen Temperaturen ist eindeutig zu bemerken. Durch Wassermessungen bei den entsprechend hohen Temperaturen kann der durch diesen Effekt verursachte Fehler mit ca. $4 \%$ angegeben werden.

Die Wandler der Zelle sind mit Bienenwachs in die Wandlerhalterung eingeklebt. Dieses hatte zur Folge, daß bei den verhältnismäßig hohen Temperaturen von > $35^{\circ} \mathrm{C}$, einige Messungen abgebrochen werde mußten, da sich die Wandler, bedingt durch das Schmelzen des Wachses, aus der Halterung gelöst hatten.

Die Piezotranslatorzelle zeigte wie die Pulszellen keine besonderen Fehler.

Die in Tabelle 6.4 angegebenen apparativen Fehler beruhen auf von Überlegungen von K. Menzel [103] und A. Rupprecht [125], wo eine detaillierte Untersuchung der auftretenden Fehler vorgenommen wird.

\begin{tabular}{|c||c|c|}
\hline Pulszelle & Bereich & rel.Gesamtfehler \\
\hline \hline \multirow{3}{*}{$1-\mathrm{MHz}$} & $\alpha<61 / \mathrm{m}$ & $3 \%$ \\
& $\alpha>61 / \mathrm{m}$ & $2 \%$ \\
& $f>30 \mathrm{MHz}$ & $2.5 \%$ \\
\hline \multirow{3}{*}{$10-\mathrm{MHz}$} & $f<50 \mathrm{MHz}$ & $1 \%$ \\
& sonst & $0.7 \%$ \\
& $f>300 \mathrm{MHz}$ & $1 \%$ \\
\hline Hyper- & $x>20 \mu \mathrm{m}$ & $2 \%$ \\
schall & sonst & $3 \%$ \\
& $<15^{\circ} \mathrm{C}$ & $7 \%$ \\
& $>35^{\circ} \mathrm{C}$ & $8 \%$ \\
\hline PZT- & $x>20 \mu \mathrm{m}$ & $<1 \%$ \\
Hyperschall & $2<x<20 \mu \mathrm{m}$ & $1.5 \%$ \\
\hline
\end{tabular}

Tabelle 6.4: Meßbereiche der verwendeten Pulszellen mit dem abgeschätzten relativen Gesamtfehlern bezgl. des $(\alpha \lambda)_{\text {gesamt }}$-Wertes und deren Geltungsbereiche nach [125] 


\section{Meßergebnisse der Ultraschallspektroskopie}

Im folgenden werden die gemessenen Ultraschallspektren der verschiedenen Lipidsuspensionen vorgestellt. Dabei stellt die Suspension DMPC/Wasser das einfachste System dar, besteht die Membran dort doch nur aus einer Lipidsorte mit einer Schmelztemperatur bei ca. $24{ }^{\circ} \mathrm{C}$. Trotzdem zeigen schon die Spektren dieser Lösung ein recht komplexes Verhalten. Meistens können die Spektren durch zwei Debye-Relaxationsprozesse und dem Bhattacharjee-Ferrell-Prozeß beschrieben werden. Wie in Kapitel 2 erläutert, weisen alle Suspensionen eine spezifische Umwandlungstemperatur auf, bei der die Membran vom gelförmigen in den fluiden Zustand wechselt. Um nun den einzelnen Relaxationsprozessen jeweils eine molekulare Ursache zuschreiben zu können, wurden von jeder Suspension Spektren bei verschiedenen Temperaturen aufgenommen. Bei der DMPC/Wasser Suspension sind es insgesamt dreizehn Stück, jedoch mit zwei unterschiedlichem Konzentrationen. Dabei sind die Meßwerte der Spektren von DMPC/Wasser bei $15{ }^{\circ} \mathrm{C}, 20{ }^{\circ} \mathrm{C}, 23.5^{\circ} \mathrm{C}, 24{ }^{\circ} \mathrm{C}, 24.5^{\circ} \mathrm{C}$, $28{ }^{\circ} \mathrm{C}$ und $32{ }^{\circ} \mathrm{C}$ und von DMPC/Cholesterin 92,5:7,5 bei S. Halsternberg [48], die Spektren DMPC/Cholesterin 95:05, 90:10 und DMPC/DPPC 83:17 bei M. Höckel [65] entnommen.

\subsection{Darstellung der Spektren}

Der Meßfequenzbereich aller Spektren reicht von ca. $180 \mathrm{kHz}$ bis 2 GHz. Die Darstellung der Spektren erfolgt entweder in der $\left(\alpha / f^{2}\right)$-Darstellung, oder sie werden in der Form

$$
(\alpha \lambda)_{\text {exc. }}=(\alpha \lambda)_{\text {gesamt }}-B \cdot f
$$

präsentiert, um die Charakteristik der Zusatzdämpfung hervorzuheben. Dabei ist der B-Wert aus der Anpaßrechnung bestimmt worden. Wegen der doppelt logarithmischen Skalierung der $(\alpha \lambda)_{\text {exc. }}$-Darstellung werden negative Werte positiv angezeigt, die allerdings dann mit einem Plus versehen $(\oplus)$ werden.

\subsubsection{Anpaßrechnungen}

In der Ultraschallspektroskopie versucht man nach der eigentlichen Messung der Spektren, von deren Verlauf und Form auf molekulare Prozesse zu schließen. Dies geschieht hier, indem theoretische Kurven von Relaxationsmodellen an die Werte der Ultraschallspektren mittels des mathematischen Algorithmus der kleinsten Quadrate von Marquardt [98] angepaßt werden.

Durch die in der Schallgeschwindigkeitsmessung und Kaloriemetrie gewonnenen Informationen über die Natur des Schmelzvorganges der Lipidmembran liegt es nahe, mit einem Fluktuationsmodell für die Ultraschalldämpfung anzusetzen. Jedoch zeigen Versuche, die Spektren mittels eines einzigen Bhattacharjee-Ferrell-Terms zu beschreiben, nur unbefriedigende Ergebnisse.

Die sich anschließenden Anpaßrechnungen mit einem zusätzlichen Debye-Term bringen schon eine wesentlich größere Übereinstimmung der theoretischen Kurve mit den 
Ultraschallspektren. Dennoch zeigt sich, daß insbesondere bei den Spektren in der Nähe der Umwandlungstemperatur ein Debye-Term definitiv nicht ausreicht.

Anpaßrechnungen mit einem Bhattacharjee-Ferrell-Term und drei Debye-Termen bringen wegen der größeren Zahl von Parametern eine gute Übereinstimmung der theoretischen Kurve mit den Meßkurven, allerdings ist die Verwendung von drei Debye-Termen nur im Einzelfall möglich. Zudem zeigt sich, daß einige wenige Spektren durchaus "nur" mit einem zusätzlichen Debye-Term anzupassen sind. In seltenen Fällen gelingt sogar eine Anpassung unter ausschließlicher Verwendung des Bhattacharjee-Ferrell-Terms. Aufgrund dieser offensichtlichen Diskrepanzen und Schwierigkeiten wird nicht wie sonst üblich von Spektrum zu Spektrum entschieden, ob die theoretische Funktion das Spektrum gut beschreibt, sondern alle Spektren werden bei den Anpaßrechung in ihrer Gesamtheit betrachtet. Dabei gelten folgende Kriterien für eine "gute" Anpassung:

Losgelöste Debye-Terme: Es erscheint unphysikalisch, daß beispielsweise ein Debye-Term bei den Spektren der Temperatur oberhalb und unterhalb des gerade betrachteten Spektrums vorhanden ist und auch in etwa die gleiche Relaxationszeit aufweist, aber bei dem gerade betrachteten Spektrum völlig verschwunden ist. So wird, falls nötig, auch ein Parameter eines Debye-Terms festgehalten.

Unsinnige BF-Parameter: Ebenso macht es wenig Sinn, wenn $\omega_{B F}$ bei einer Temperatur weit außerhalb der Umwandlungstemperatur fast Null ist, obwohl dieses jedoch nur bei $T_{m}$ sinnvoll erscheint.

Stetigkeit der Parameter: Alle Paremeter, sowohl die des Bhattacharjee-FerrellTerms als auch die der Debye-Terme, müssen im Rahmen der Fehler eine stetigen Verlauf über der Temperatur aufweisen.

Die zur Umrechnung der $\alpha / f^{2}$-Daten in die $(\alpha \cdot \lambda)$-Daten notwendige Schallgeschwindigkeit ist aus den entsprechenden Messungen dazu hinlänglich bekannt. Dispersion kann über dem betrachteten Frequenzbereich vernachlässigt werden, da selbst bei der Suspension mit der höchsten Konzentration an der Umwandlungstemperatur die auftretende Dämpfung zu einer Dispersionsstufe von weniger als 0,5\% führt. Dieser Fehler ist gegenüber den apparativen Fehlern zu vernachlässigen.

Fast alle Spektren können schließlich mittels einer Spektralfunktion bestehend aus einem Bhattacharjee-Ferrell-Term und zwei Debye-Termen hinreichend beschrieben werden:

$$
(\alpha \lambda)_{g e s a m t}=A_{B F} \omega^{-0.0572} F(\Omega)+\sum_{i=1}^{2} A_{i} \cdot \frac{\omega \tau_{i}}{1+\left(\omega \tau_{i}\right)^{2}}-B \cdot f .
$$

Lediglich bei den Suspensionen DMPC/Cholesterin 85:15 und 66:33 reicht ein Bhattacharjee-Ferrell-Term und nur ein Debye-Term zur Beschreibung der Spektren aus:

$$
(\alpha \lambda)_{\text {gesamt }}=A_{B F} \omega^{-0.0572} F(\Omega)+A_{1} \cdot \frac{\omega \tau_{1}}{1+\left(\omega \tau_{1}\right)^{2}}-B \cdot f
$$




\subsubsection{Festlegung von Parametern}

Bei fast allen Spektren können die Parameter der Spektralfunktion durch völlig freie Anpassung gewonnen werden. Die spezielle Form der angepaßten Spektralfunktion, insbesondere die Zusammenfassung des hochfrequenten Anteils außerhalb des gemessenen Spektrums durch den Term $B \cdot f$, führt bei einigen Spektren zu Problemen bei den Anpaßrechnungen. Liegt einer der beiden Debye-Terme am oberen Ende des gemessenen Spektrums, so kann dieser Prozeß unter Umständen nicht mehr eindeutig festgelegt werden. Dieses führt bei freier Anpassung dazu, daß der Debye-Term in dem hochfrequenten Bereich außerhalb des Spektrums mit sehr hoher Amplitude gelegt wird und der B-Wert gegen Null tendiert. Um diese unrealistischen und unphysikalischen Werte zu verhindern, muß bei einigen Spektren während der Anpassung der Spektralfunktion der B-Wert festgehalten werden. Dabei wird allerdings die Höhe des B-Wertes aus einer Interpolation aus Werten der Spektren anderer Temperaturen ermittelt, um eine möglichst realistische Größe zu erhalten.

Falls nötig, wird in Einzelfällen auch ein Parameter eines Debye-Terms festgehalten. Auch hier wird der Wert so gewählt, daß er im Vergleich zu den Werten bei anderen Temperaturen realistisch erscheint.

Festgehaltene Parameter sind durch den Kursivdruck in den Tabellen der Parameter (Tabelle 7.1, 7.2, 7.3 und 7.4) speziell gekennzeichnet.

\subsubsection{DMPC/Wasser}

In den folgenden Abbildungen 7.1, 7.2, 7.5, 7.6 und 7.7 sind die Spektren der DMPC/Wasser Suspensionen bei verschiedenen Temperaturen einmal in der $\left(\alpha / f^{2}\right)$ Darstellung und in der $(\alpha \lambda)_{\text {exc. }}$-Darstellung aufgeführt. Beim Vergleich der Spektren untereinander ist zu beachten, daß die Suspension DMPC $2 \frac{\mathrm{mg}}{\mathrm{ml}}$ bei den Temperaturen $18{ }^{\circ} \mathrm{C}, 22{ }^{\circ} \mathrm{C}, 25{ }^{\circ} \mathrm{C}, 26^{\circ} \mathrm{C}, 27^{\circ} \mathrm{C}$ und $30^{\circ} \mathrm{C}$ und die DMPC $10 \frac{\mathrm{mg}}{\mathrm{ml}}$ Lösung bei den Temperaturen $15{ }^{\circ} \mathrm{C}, 20^{\circ} \mathrm{C}, 23.5^{\circ} \mathrm{C}, 24{ }^{\circ} \mathrm{C}, 24.5^{\circ} \mathrm{C}, 28^{\circ} \mathrm{C}$ und $32{ }^{\circ} \mathrm{C}[48]$ gemessen wurde.

Abb. 7.3 zeigt das Spektrum der DMPC $10 \frac{\mathrm{mg}}{\mathrm{ml}}$ Suspension bei $15{ }^{\circ} \mathrm{C}$ mit angepaßter Spektralfunktion. Zur Verdeutlichung des Verhältnisses der Amplitude der einzelnen Prozesse untereinander sind in dieser Abbildung außer der gesamten Spektralfunktion (durchgezogene Linie) auch die einzelnen Prozesse (gestrichelte Linien) dargestellt. Man sieht ganz deutlich die Dominanz des Bhattacharjee-Ferrell-Term bei der Beschreibung der Dämpfung. Die zwei Einzelzeitrelaxationen bezitzen im Verhältnis dazu eine fast verschwindend geringe Amplitude. In Abb. 7.4 ist das Spektrum der gleichen Suspension bei $24{ }^{\circ} \mathrm{C}$ in der gleichen Darstellungsform zu sehen. Die zwei Debye-Terme haben im Vergleich zur Abb. 7.3 eine größere Amplitude, doch auch hier dominiert eindeutig der Bhattacharjee-Ferrell-Term.

Betrachtet man alle gemessenen Spektren der DMPC/Wasser Suspensionen verschiedener Temperaturen in der $(\alpha \lambda)_{\text {exc. }}$-Darstellung untereinander, so fällt sofort die starke Formänderung der Spektren mit der Änderung der Temperatur auf. Die beiden Einzelzeitrelaxationen sind unterhalb des Umwandlungsbereiches getrennt voneinander im hochfrequenten Teil des Spektrum zu finden. In der Umwandlung wandern diese beiden Prozesse zusammen in den tieffrequenten Bereich und liegen dort derart dicht beieinander, daß sie kaum voneinander zu trennen sind. Oberhalb der Umwandlungstemperatur liegen die beiden Debye-Terme erneut im hochfrequen- 


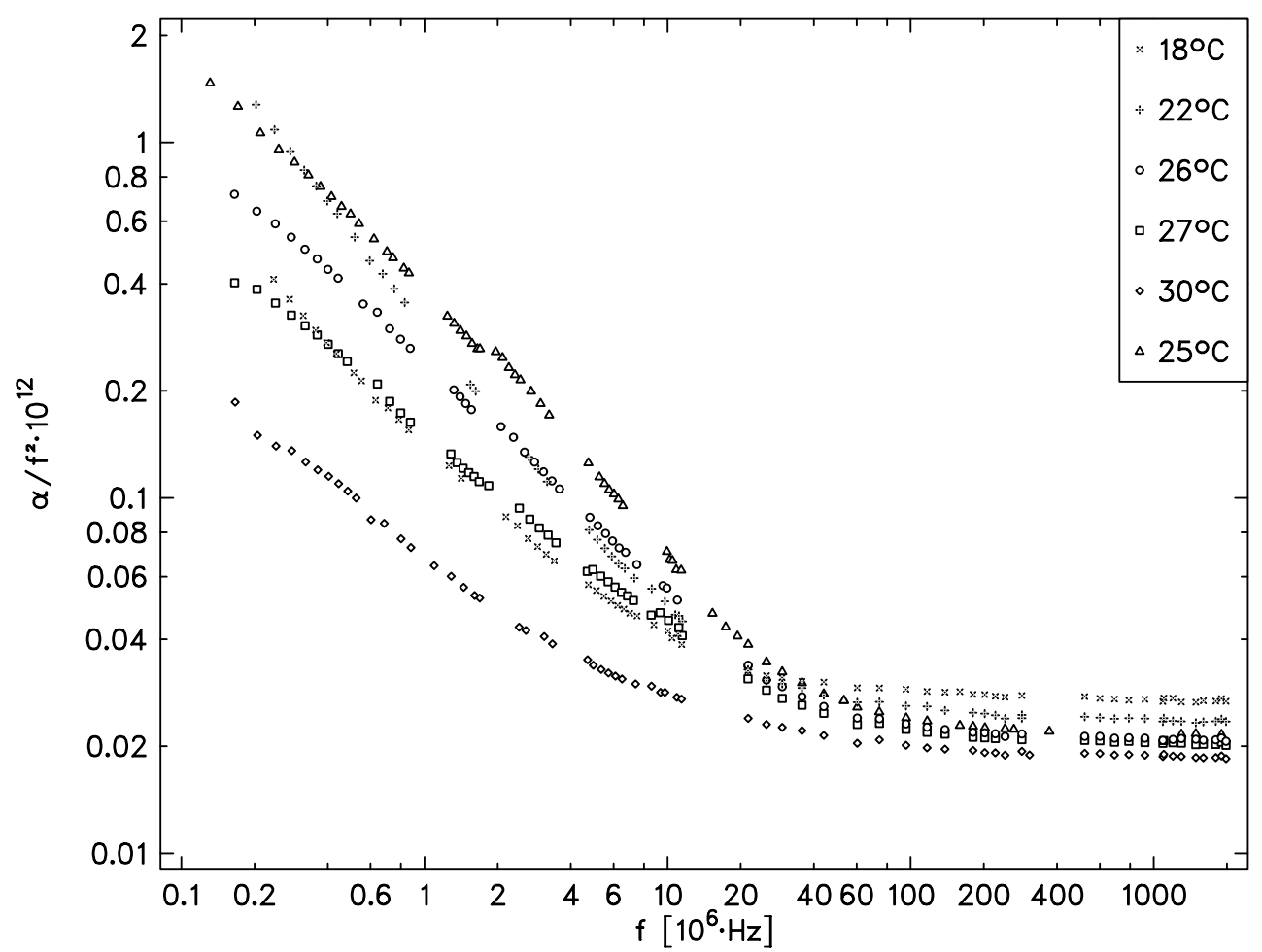

Abb. 7.1: Ultraschallspektren der DMPC $2 \frac{\mathrm{mg}}{\mathrm{ml}}$ Suspensionen in der $\alpha / f^{2}$-Darstellung

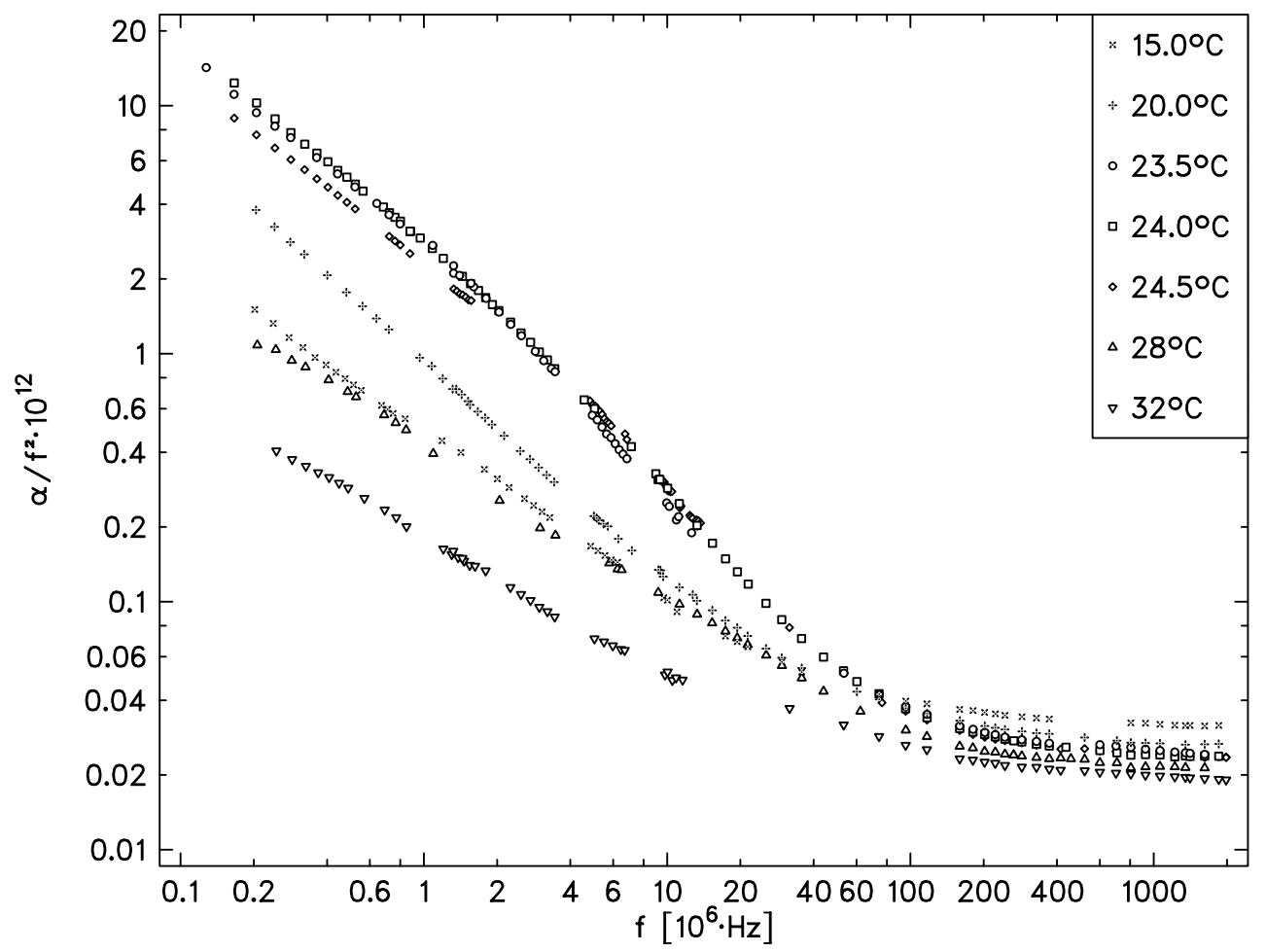

Abb. 7.2: Ultraschallspektren der DMPC $10 \frac{\mathrm{mg}}{\mathrm{ml}}$ Suspensionen in der $\alpha / f^{2}$-Darstellung 


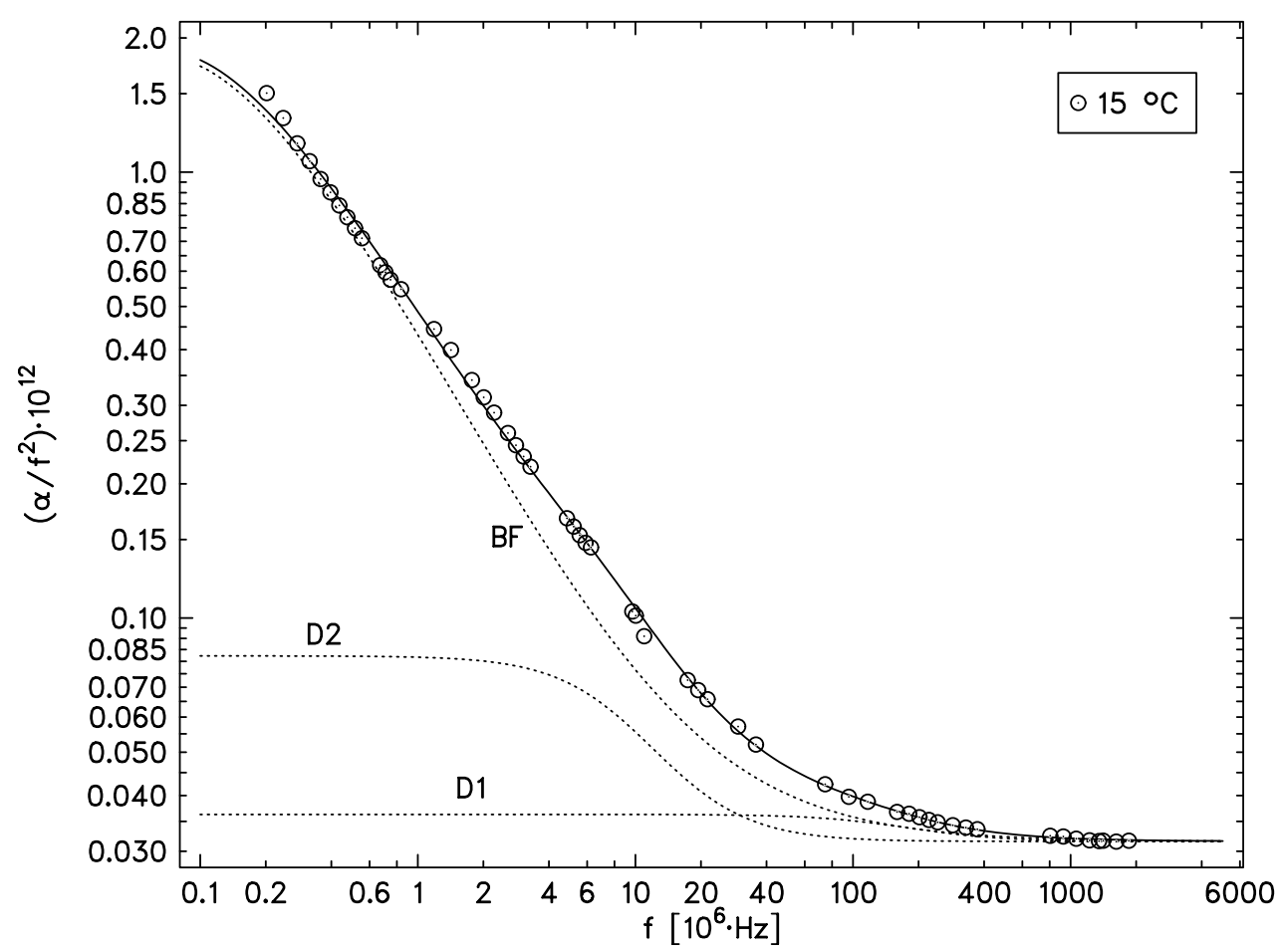

Abb. 7.3: Ultraschallspektrum der DMPC $10 \frac{\mathrm{mg}}{\mathrm{ml}}$ Suspensionen in der $\alpha / f^{2}$-Darstellung bei $15{ }^{\circ} \mathrm{C}$; die durchgezogene Linie stellt die angepaßte Spektralfunktion dar und die gestrichelten Linien die Beiträge der einzelnen Relaxationsterme

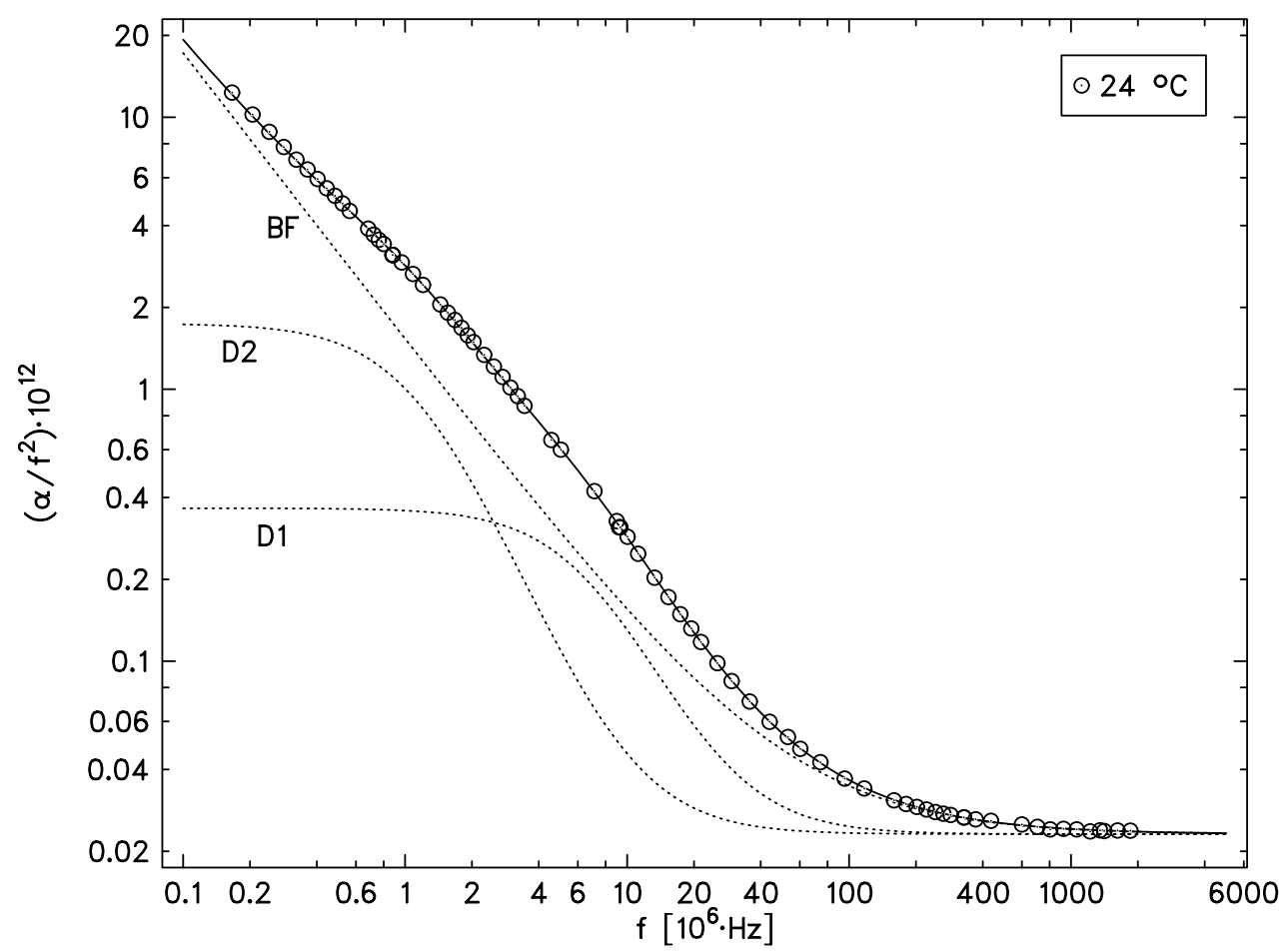

Abb. 7.4: Ultraschallspektrum der DMPC $10 \frac{\mathrm{mg}}{\mathrm{ml}}$ Suspensionen in der $\alpha / f^{2}$-Darstellung bei $24{ }^{\circ} \mathrm{C}$; die durchgezogene Linie stellt die angepaßte Spektralfunktion dar und die gestrichelten Linien die Beiträge der einzelnen Relaxationsterme 

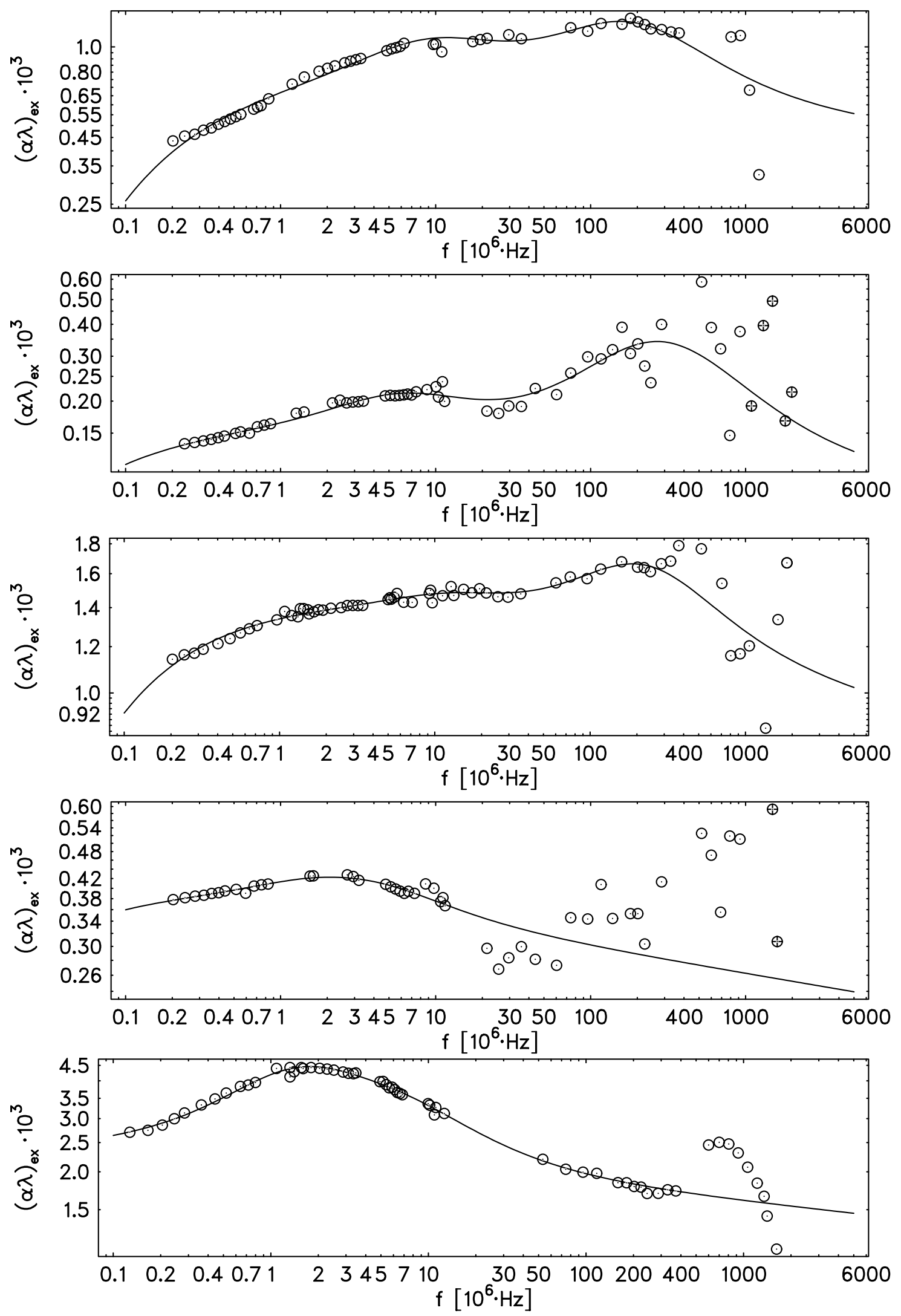

Abb. 7.5: Ultraschallspektren der DMPC Suspensionen; Konzentration und Temperatur von oben nach unten: $10 \frac{\mathrm{mg}}{\mathrm{ml}} 15^{\circ} \mathrm{C}, 2 \frac{\mathrm{mg}}{\mathrm{ml}} 18^{\circ} \mathrm{C}$, $10 \frac{\mathrm{mg}}{\mathrm{ml}} 20{ }^{\circ} \mathrm{C}, 2 \frac{\mathrm{mg}}{\mathrm{ml}} 22{ }^{\circ} \mathrm{C}, 10 \frac{\mathrm{mg}}{\mathrm{ml}} 23,5{ }^{\circ} \mathrm{C}$ mit angepaßter Spektralfunktion (7.2) 

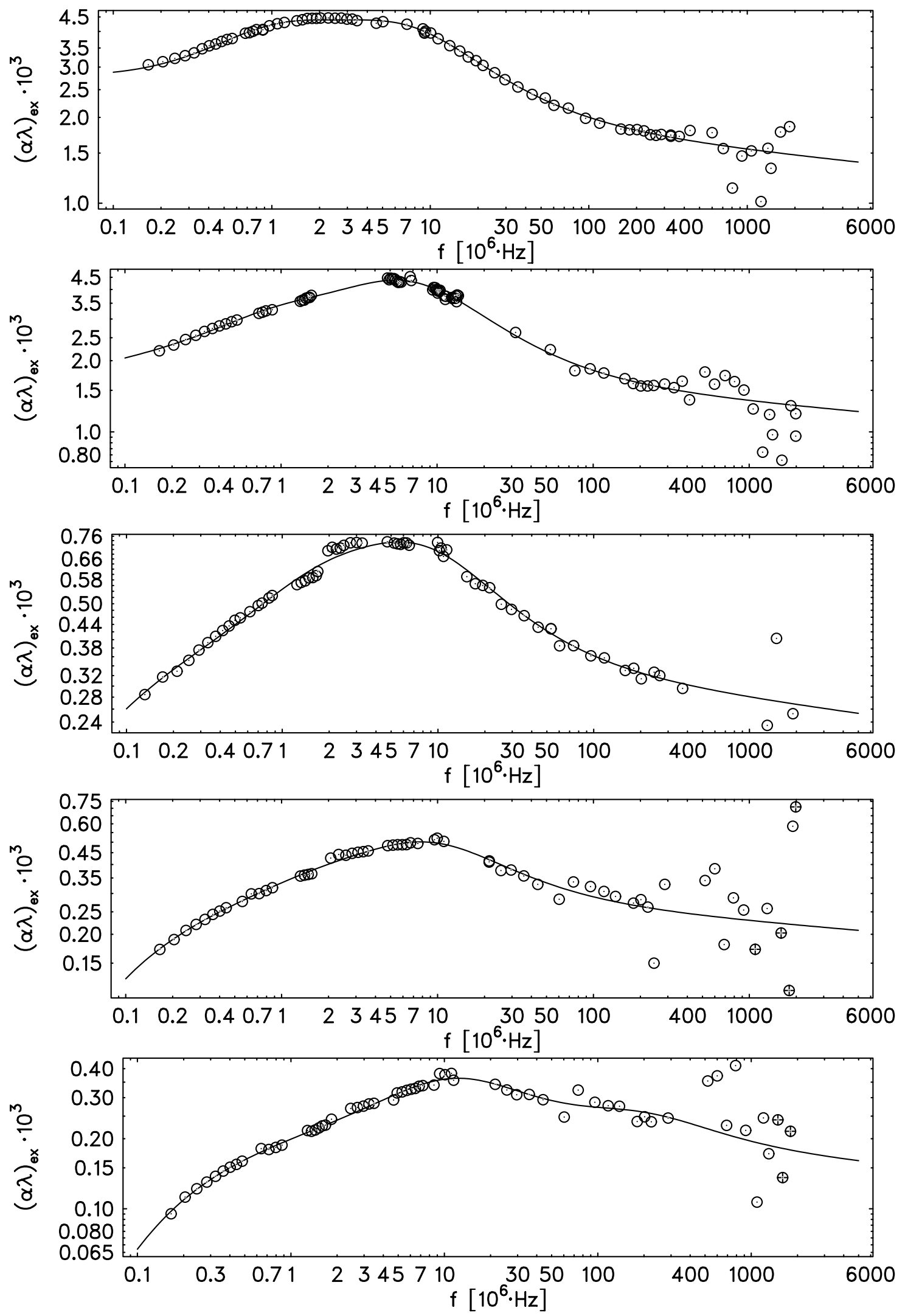

Abb. 7.6: Ultraschallspektren der DMPC Suspensionen; Konzentration und Temperatur von oben nach unten: $10 \frac{\mathrm{mg}}{\mathrm{ml}} 24^{\circ} \mathrm{C}, 10 \frac{\mathrm{mg}}{\mathrm{ml}} 24,5$ ${ }^{\circ} \mathrm{C}, 2 \frac{\mathrm{mg}}{\mathrm{ml}} 25{ }^{\circ} \mathrm{C}, 2 \frac{\mathrm{mg}}{\mathrm{ml}} 26{ }^{\circ} \mathrm{C}, 2 \frac{\mathrm{mg}}{\mathrm{ml}} 27{ }^{\circ} \mathrm{C}$ mit angepaßter Spektralfunktion (7.2) 

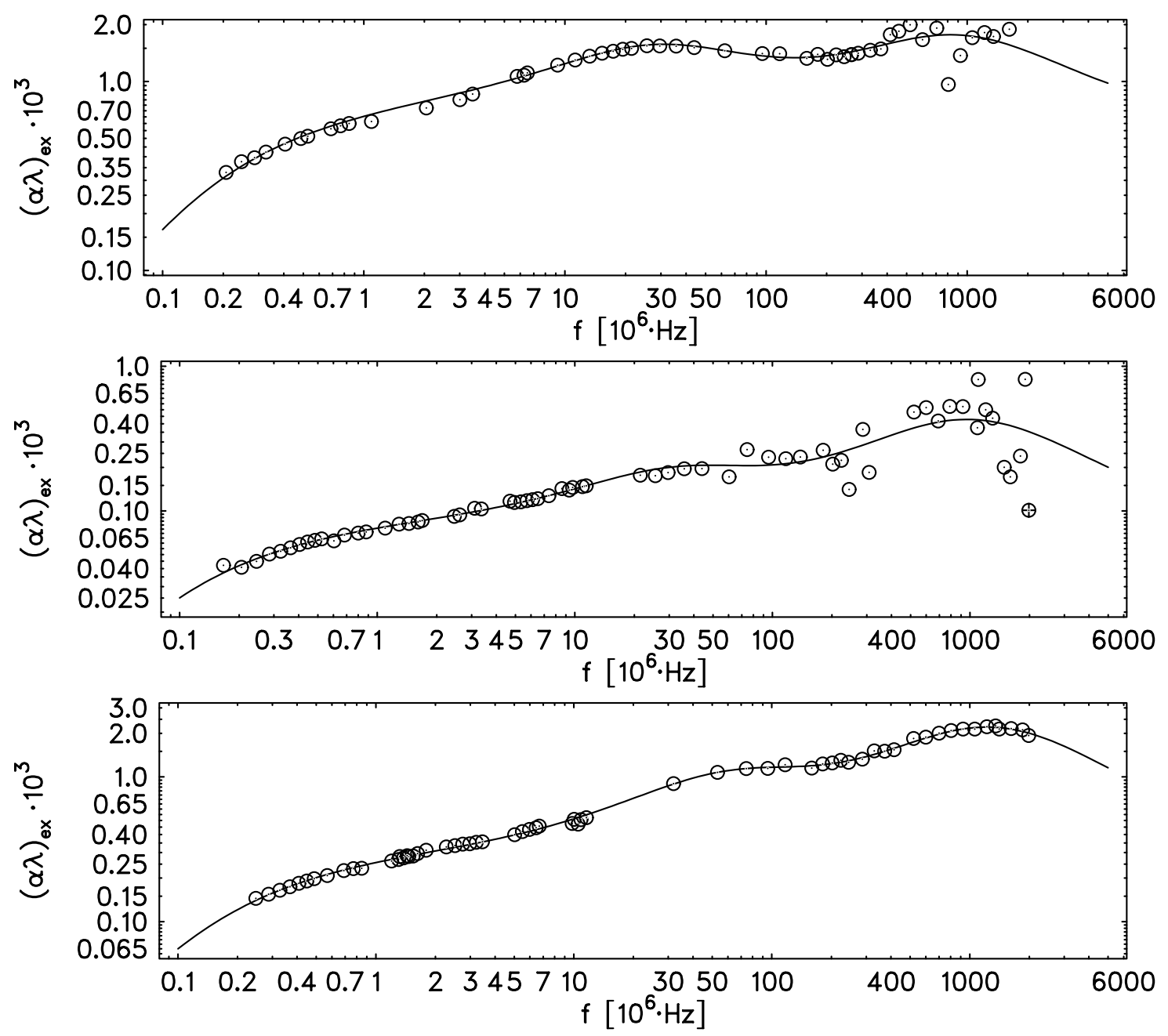

Abb. 7.7: Ultraschallspektren der DMPC Suspensionen; Konzentration und Temperatur von oben nach unten: $10 \frac{\mathrm{mg}}{\mathrm{ml}} 28^{\circ} \mathrm{C}, 2 \frac{\mathrm{mg}}{\mathrm{ml}} 30^{\circ} \mathrm{C}$, $10 \frac{\mathrm{mg}}{\mathrm{ml}} 32{ }^{\circ} \mathrm{C}$ mit angepaßter Spektralfunktion (7.2) 


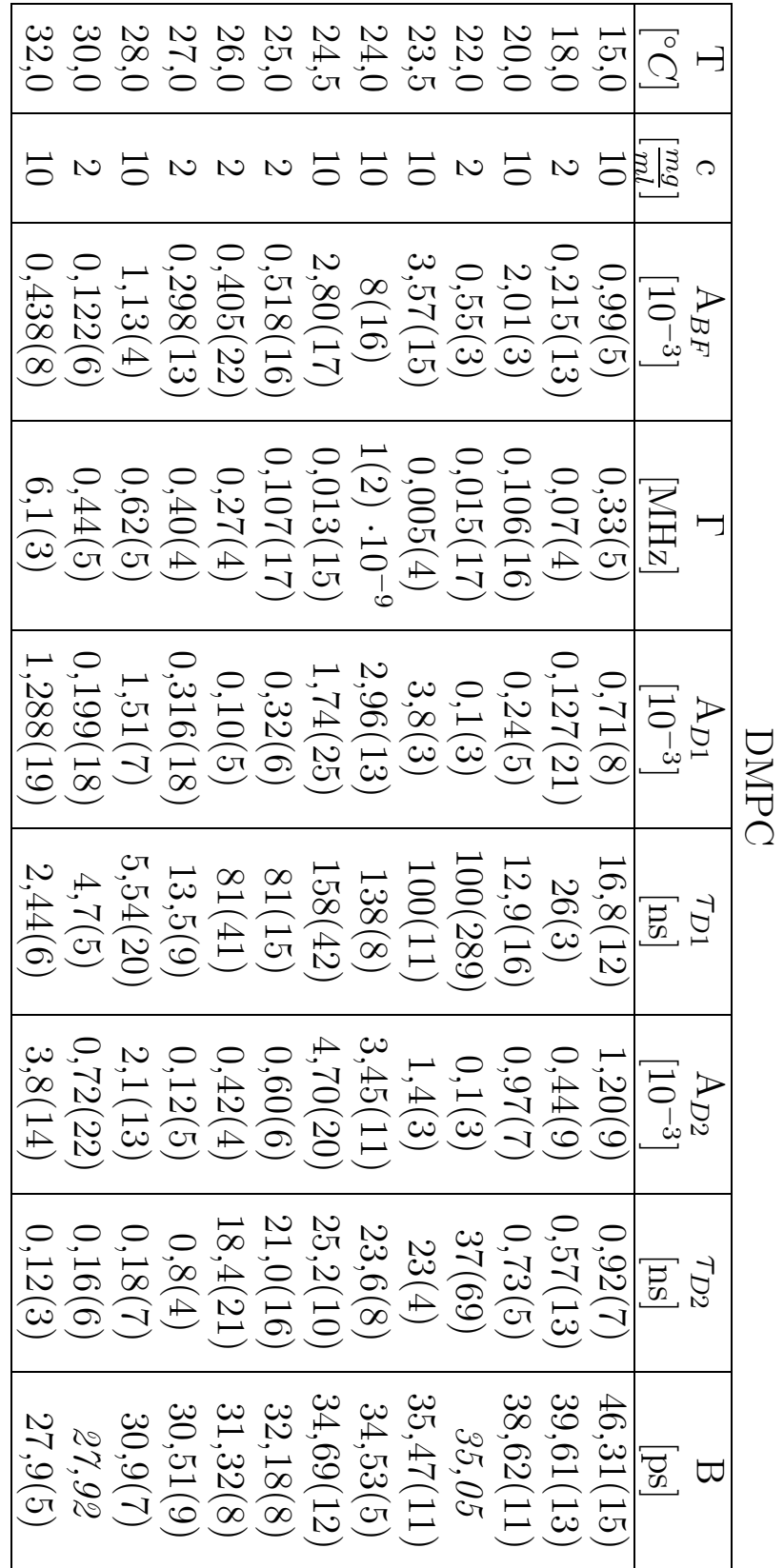

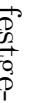


ten Teil des Spektrums. Die Parameter der angepaßten Spektralfunktion sind in Tabelle 7.1 aufgeführt.

\subsubsection{DMPC/Cholesterin 95:05}

In Abb. 7.8 folgen die Spektren der DMPC/Cholesterin 95:05 Suspension bei drei verschiedenen Temperaturen mit der Konzentration $10 \frac{\mathrm{mg}}{\mathrm{ml}}$ (Meßpunkte aus [65]). Deutlich ist eine starke Formänderung der Spektren und ein ähnlicher Gang der Debye-Terme bei veränderter Temperatur wie bei reinem DMPC zu sehen. Offensichtlich scheint die "geringe" Zugabe von nur $5 \%$ Cholesterin die Eigenschaften der Membran nicht merklich zu beeinflussen.
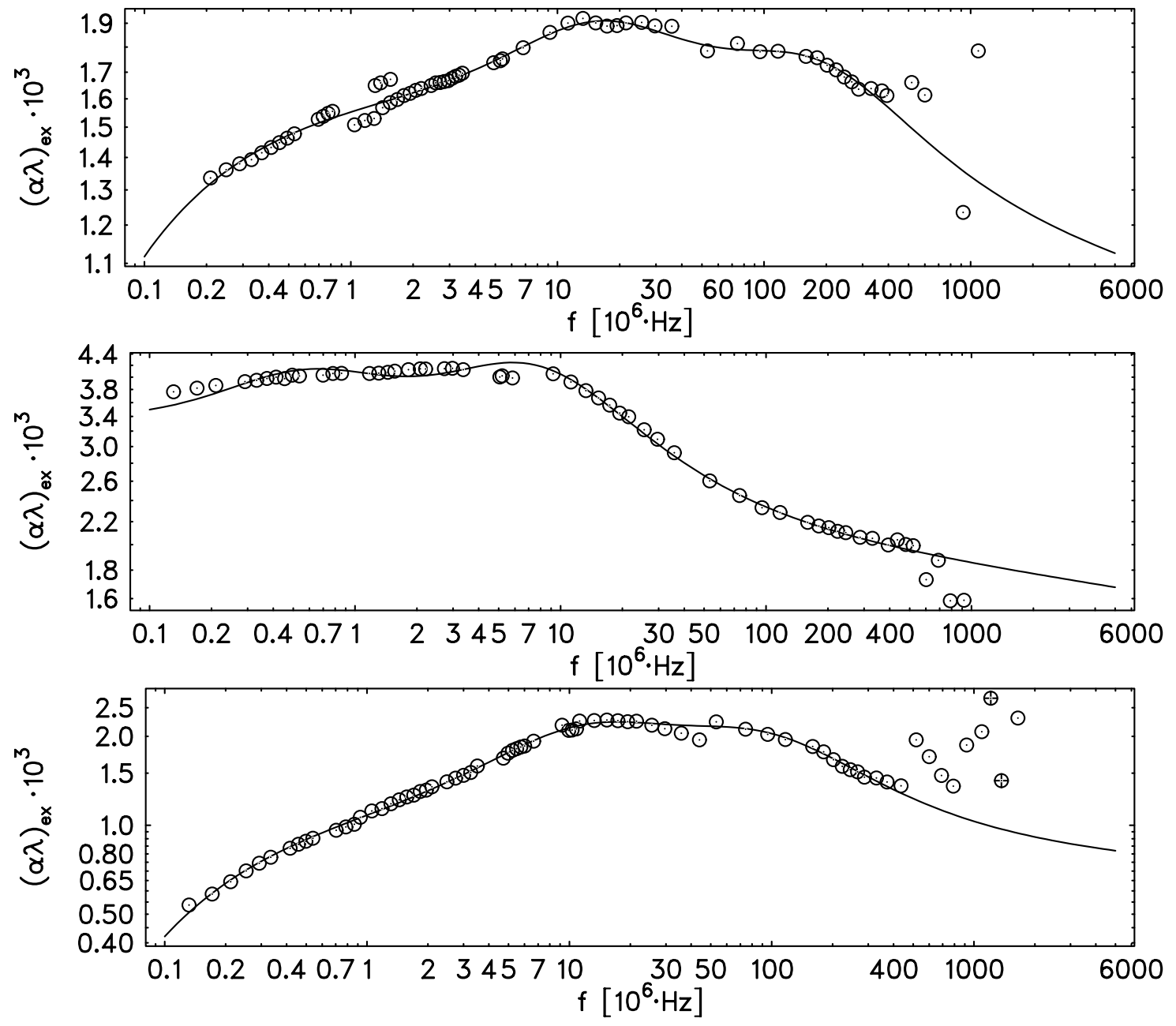

Abb. 7.8: Ultraschallspektren der DMPC/Cholesterin 95:05 $10 \frac{\mathrm{mg}}{\mathrm{ml}}$ Suspensionen; Temperatur von oben nach unten: $20{ }^{\circ} \mathrm{C}, 24{ }^{\circ} \mathrm{C}, 28^{\circ} \mathrm{C}$ mit angepaßter Spektralfunktion (7.2) 


\subsubsection{DMPC/Cholesterin 92.5:7.5}

Abb. 7.9 zeigt die Spektren der DMPC/Cholesterin 92.5:7.5 $10 \frac{\mathrm{mg}}{\mathrm{ml}}$ Suspension bei drei verschiedenen Temperaturen (Meßpunkte aus [48]). Auch hier ist die Dynamik des Schmelzprozesses der Membran durch die charakteristischen Veränderungen im Ultraschallspektrum mit der Temperatur zu entdecken.
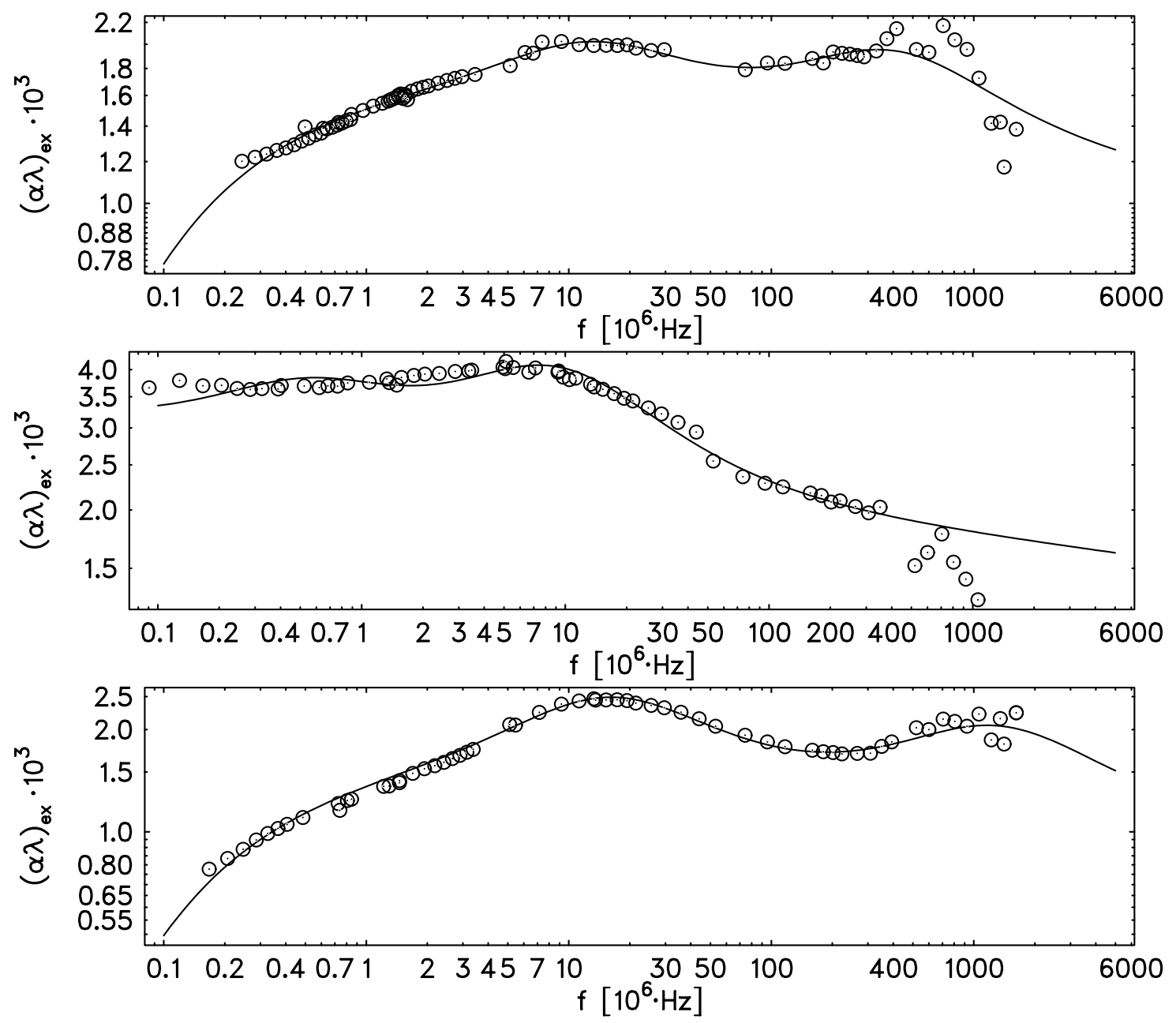

Abb. 7.9: Ultraschallspektren der DMPC/Cholesterin 92,5:7,5 $10 \frac{\mathrm{mg}}{\mathrm{ml}}$ Suspensionen; Temperatur von oben nach unten: $20^{\circ} \mathrm{C}, 24{ }^{\circ} \mathrm{C}, 28^{\circ} \mathrm{C}$ mit angepaßter Spektralfunktion (7.2)

\subsubsection{DMPC/Cholesterin 90:10}

Die Spektren der DMPC/Cholesterin 90:10 Suspension bei drei verschiedenen Temperaturen mit der Konzentration $10 \frac{\mathrm{mg}}{\mathrm{ml}}$ (Meßpunkte aus [65]) sind in der Abb. 7.10 dargestellt. Auch bei dieser Zusammensetzung der Membran unterliegen die Ultraschallspektren einer Formänderung. Dennoch ist eine Änderung der Spektren im Vergleich zu den anderen DMPC/Cholesterin Mischungen und zu den Spektren der DMPC/Wasser Suspensionen zu erkennen. Die Parameter aus den Anpassungsrechnungen sind in Tabelle 7.2 aufgeführt. 

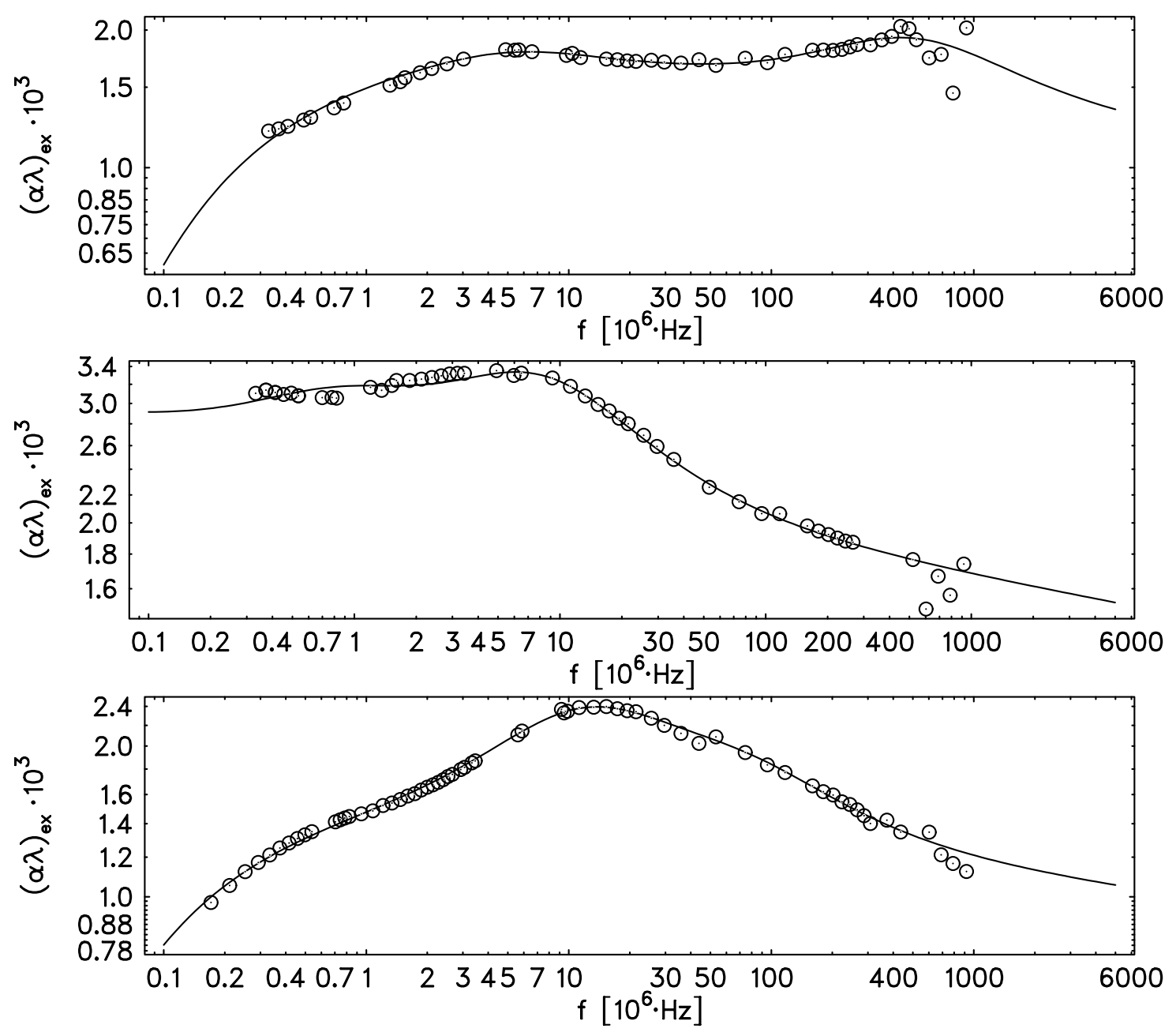

Abb. 7.10: Ultraschallspektren der DMPC/Cholesterin 90:10 $10 \frac{\mathrm{mg}}{\mathrm{ml}}$ Suspensionen; Temperatur von oben nach unten: $20^{\circ} \mathrm{C}, 24{ }^{\circ} \mathrm{C}, 28^{\circ} \mathrm{C}$ mit angepaßter Spektralfunktion (7.2)

\subsubsection{DMPC/Cholesterin 85:15}

Mit steigendem Cholesteringehalt sollte nach den Wärmekapazitäts- und Schallgeschwindigkeitsmessungen die charakteristische Umwandlung der Membran bei 24 ${ }^{\circ} \mathrm{C}$ zunehmend verschwinden. Um die molekularen Vorgänge in der Lipiddoppelschicht zu verstehen, wurden auch Suspensionen mit vergleichsweise hohem Cholesteringehalt in der Ultraschallspektroskopie gemessen. Abb. 7.11 zeigt die Spektren der DMPC/Cholesterin 85:15 $2 \frac{\mathrm{mg}}{\mathrm{ml}}$ Suspension bei fünf verschiedenen Temperaturen. Man sieht deutlich, daß sich kaum noch Veränderungen des Spektrums bei veränderter Temperatur ergeben. Schaut man genau hin, so entdeckt man eine leichte Verschiebung des mittelfrequenten Debye-Prozesses und des Bhattacharjee-FerrellProzesses.

\subsubsection{DMPC/Cholesterin 66:33}

Abb. 7.12 zeigt die Spektren der DMPC/Cholesterin 66:33 $2 \frac{\mathrm{mg}}{\mathrm{ml}}$ Suspension bei fünf verschiedenen Temperaturen. Nun ist aufgrund des hohen Cholesteringehaltes die charakteristische Umwandlung völlig verschwunden und alle fünf Spektren zeigen 


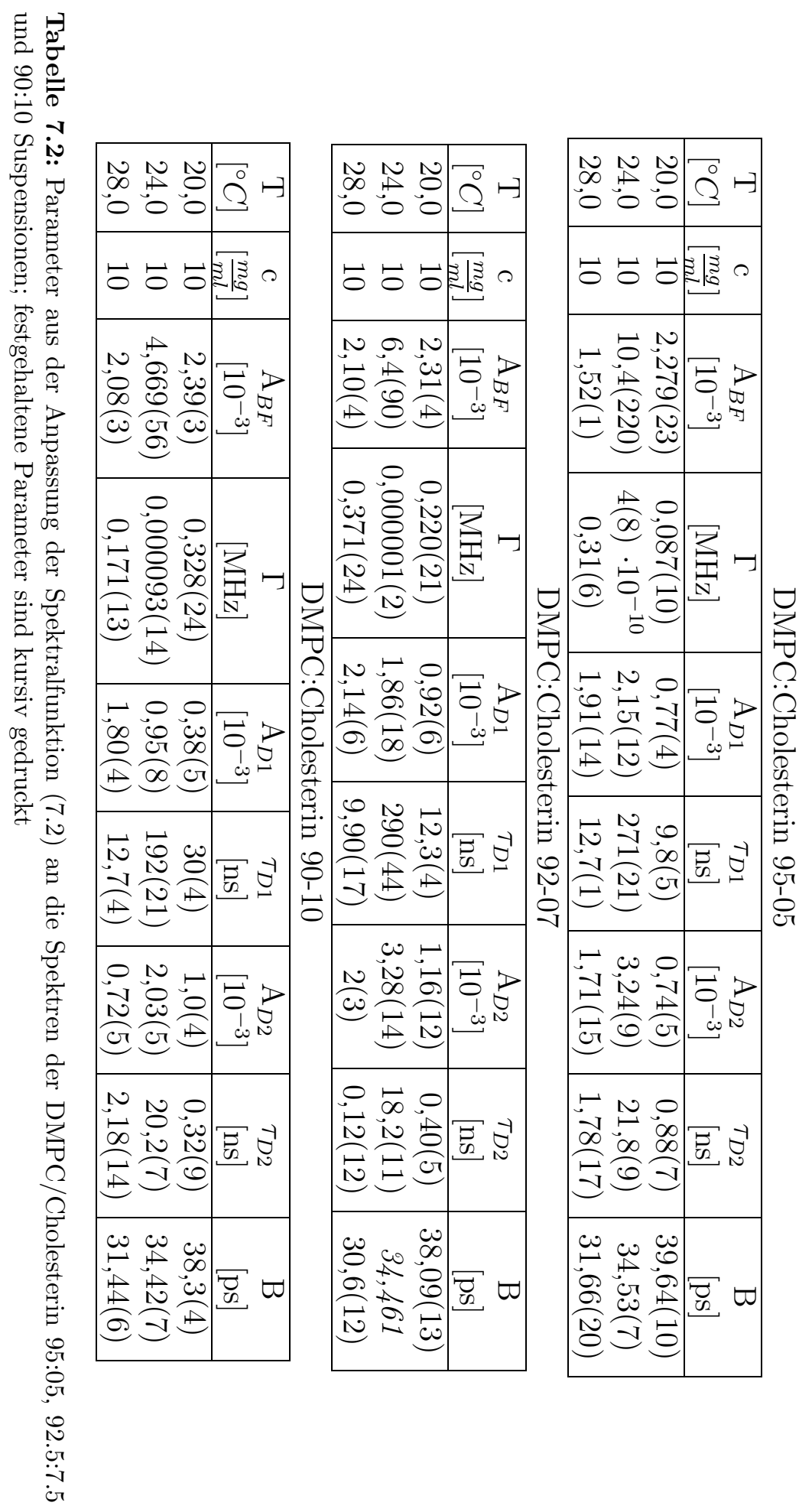



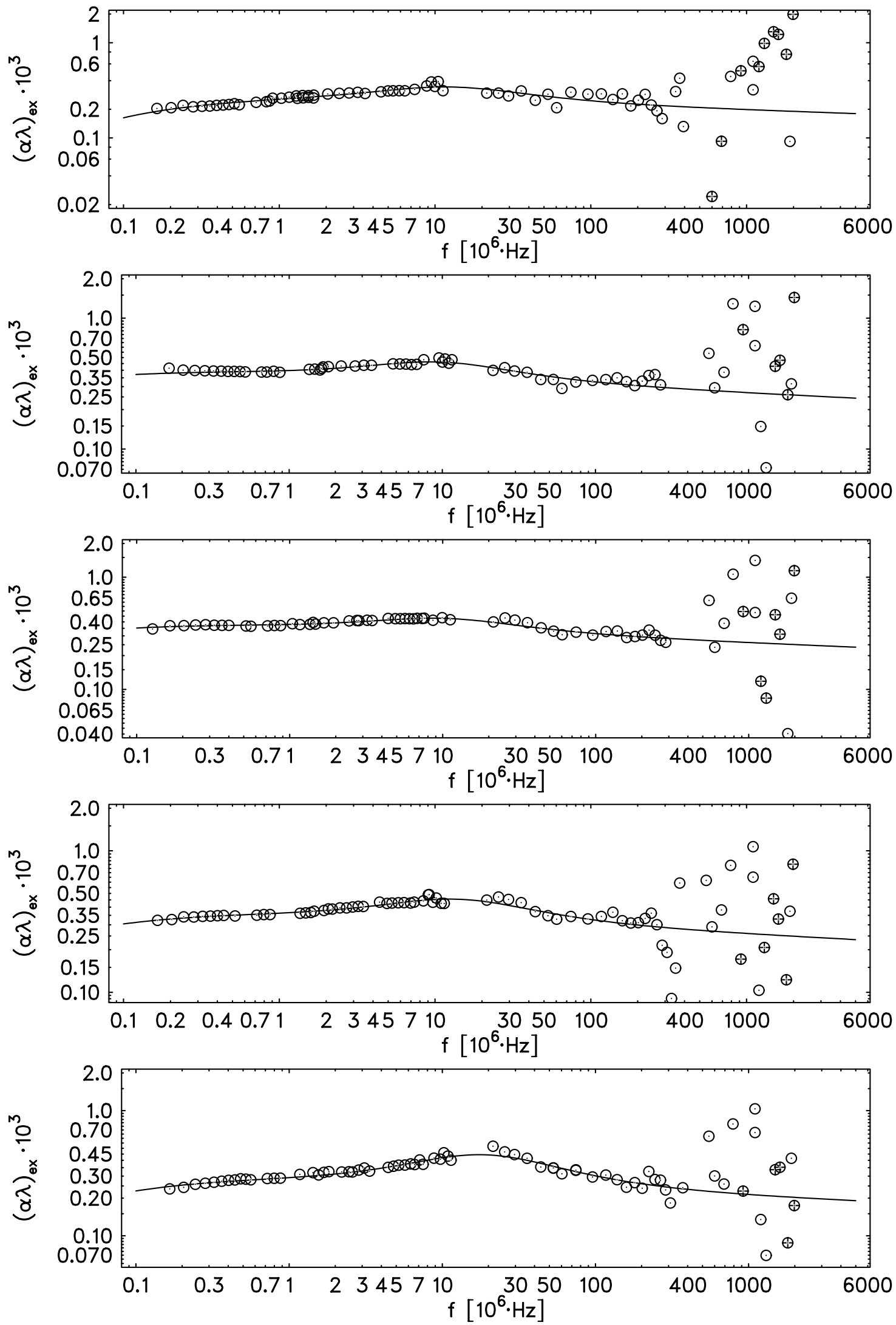

Abb. 7.11: Ultraschallspektren der DMPC/Cholesterin 85:15 $2 \frac{\mathrm{mg}}{\mathrm{ml}}$ Suspensionen; Temperatur von oben nach unten: $20{ }^{\circ} \mathrm{C}, 23{ }^{\circ} \mathrm{C}, 24{ }^{\circ} \mathrm{C}$, $25^{\circ} \mathrm{C}, 28^{\circ} \mathrm{C}$ mit angepaßter Spektralfunktion (7.3) 

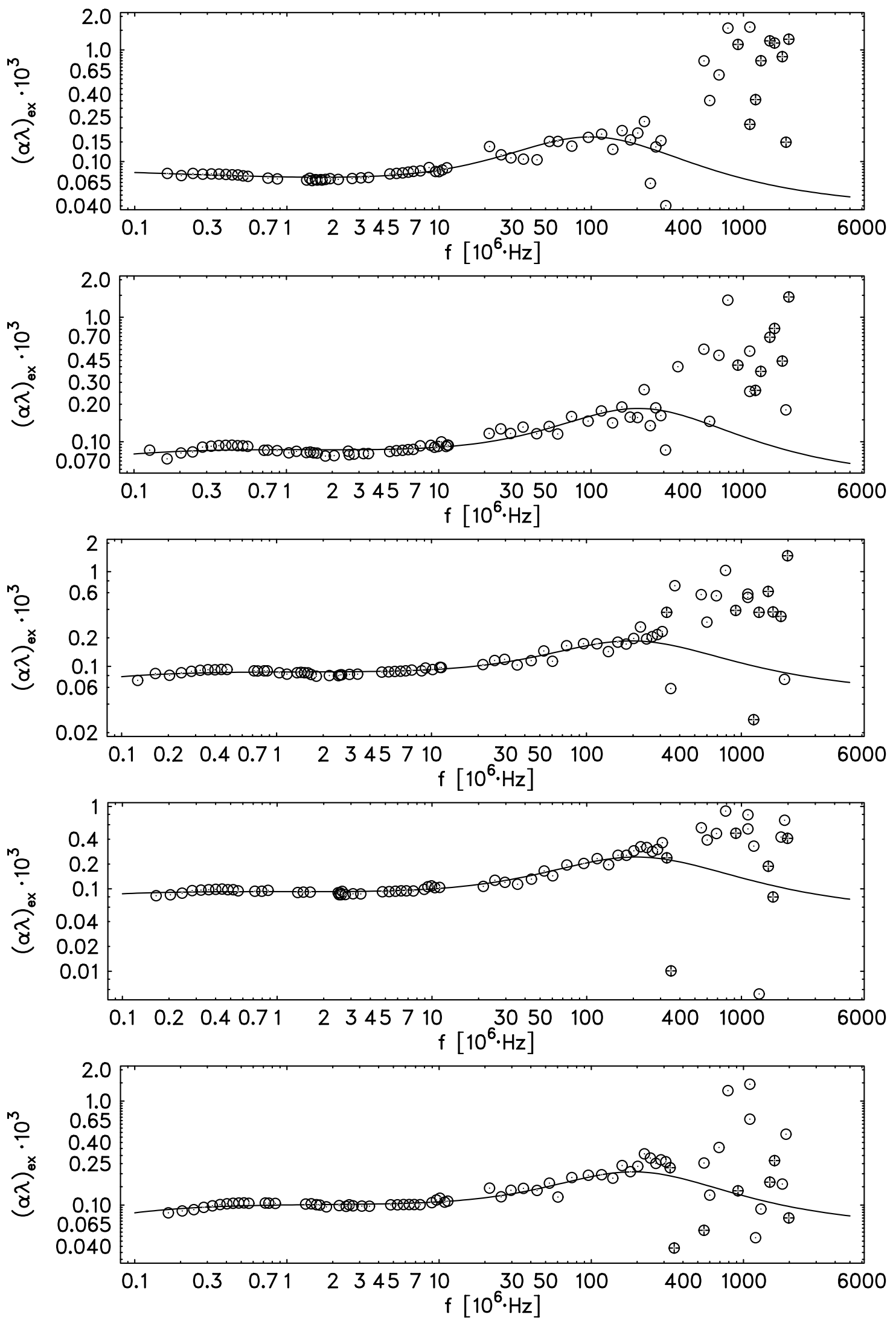

Abb. 7.12: Ultraschallspektren der DMPC/Cholesterin 66:33 $2 \frac{\mathrm{mg}}{\mathrm{ml}}$ Suspensionen; Temperatur von oben nach unten: $20^{\circ} \mathrm{C}, 23^{\circ} \mathrm{C}, 24^{\circ} \mathrm{C}$, $25{ }^{\circ} \mathrm{C}, 28{ }^{\circ} \mathrm{C}$ mit angepaßter Spektralfunktion (7.3) 


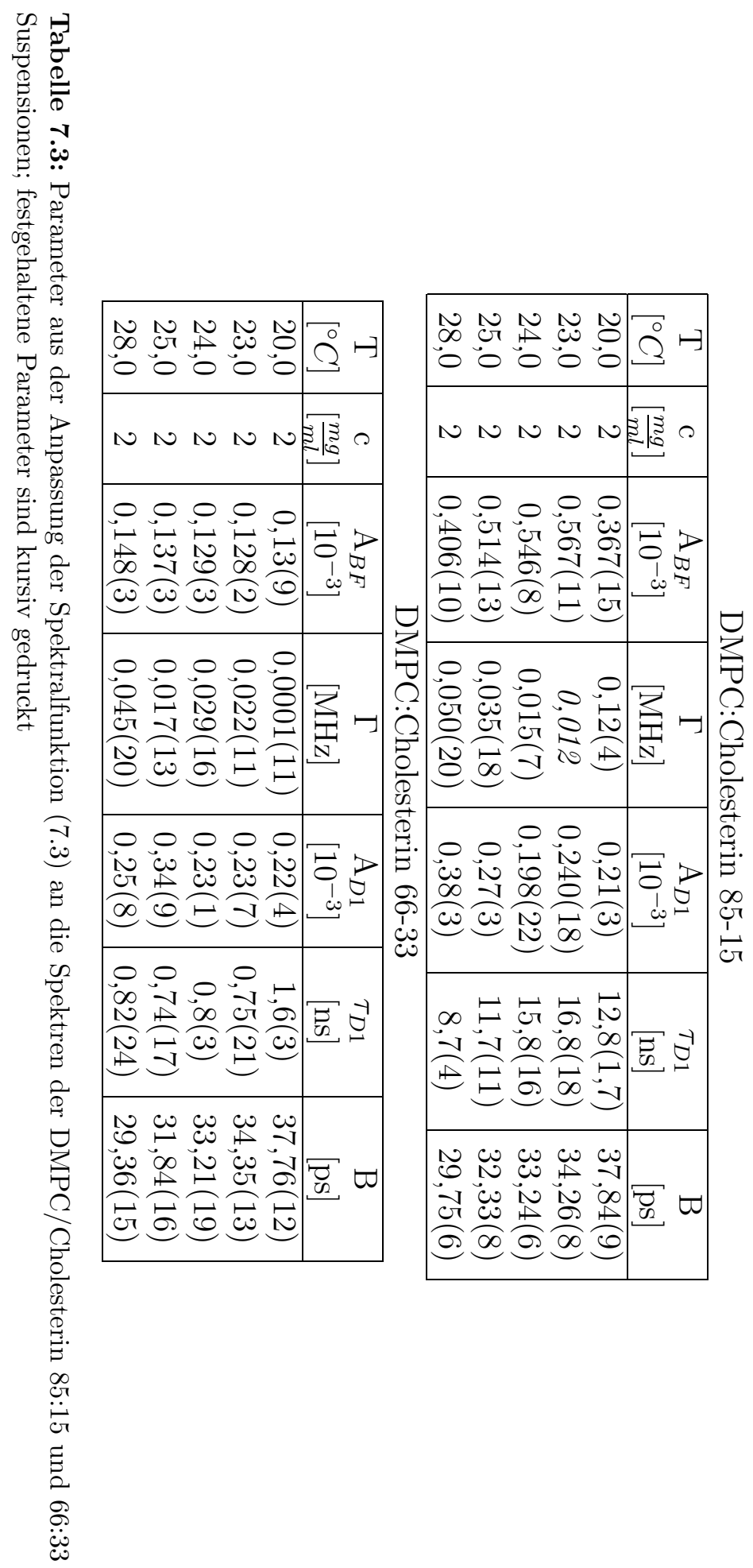


fast den gleichen Verlauf. Die Parameter aus den Anpassungsrechnungen sind in Tabelle 7.3 aufgeführt.

\subsubsection{DMPC/DPPC 83:17}

In der folgenden Abb. 7.13 sind die Spektren der DMPC/DPPC 83:17 $2 \frac{\mathrm{mg}}{\mathrm{ml}}$ Suspension dargestellt (Meßpunkte aus [65]). Da die Temperatur der Hauptumwandlung um ca. $27^{\circ} \mathrm{C}$ zu erwarten ist, wurden die Temperaturen, bei denen die Ultraschallspektren aufgenommen wurden, entsprechend angepaßt. Der Vergleich mit den Spektren der DMPC/Wasser Suspensionen zeigt ein ähnliches Verhalten der Spektren über der Temperatur insbesondere der Debye-Prozesse.

\subsubsection{DMPC/DPPC 50:50}

Es folgt noch die Darstellung der Spektren der Suspensionen DMPC/DPPC 50:50 $2 \frac{m g}{m l}$ in Abb. 7.14 .

\subsubsection{DMPC/DPPC 17:83}

Schließlich folgen noch die Spektren der Suspensionen DMPC/DPPC 83:17 $2 \frac{\mathrm{mg}}{\mathrm{ml}}$ in Abb. 7.15. Die Parameter aus den Anpassungsrechnungen an die DMPC/DPPC Suspensionen sind in Tabelle 7.4 aufgeführt.

\subsubsection{Fehler der Parameter}

In den Tabellen 7.1, 7.2, 7.3 und 7.4 sind die aus den Anpaßrechnung gewonnenen Parameter der Spektralfunktion (7.2) mit den entsprechenden Fehlern aufgeführt. Die Fehler ergeben sich in erster Linie aus der Streuung der $(\alpha \cdot \lambda)$-Meßwerte und deren Abweichung von der angepaßten Spektralfunktion. Auffällig in diesem Zusammenhang sind die sehr hohen Fehler des Bhattacharjee-Ferrell-Terms im Bereich der Umwandlungstemperatur $T_{c}$. Aufgrund des hohen, aber dennoch eingeschränkten Frequenzbereiches für die Messung der Ultraschallspektren ist es hier nicht möglich, die Charakteristik der Ultraschalldämpfung bei $T_{c}$ vollständig auszumessen. Dazu müßten Meßwerte bei sehr tiefen Frequenzen (einige $\mathrm{Hz}$ ) aufgenommen werden. Hier steht zur Interpretation "nur" der vergleichsweise hochfrequente Teil ab $200 \mathrm{kHz}$ zur Verfügung. Dennoch sind durch Anpaßrechnungen der Spektralfunktion über den Verlauf der gemessenen Punkte Aussagen über die Relaxationsrate möglich, dieses jedoch mit entsprechend hohem Fehler. So sind die Relaxationsraten und die Amplituden des Bhattacharjee-Ferrell-Terms bei $T_{c}$ mit einem sehr hohen Fehler behaftet. Dennoch kann die durch die Anpaßrechnungen gewonnene Größenordung dieser Parameter als interpretationsfähig betrachtet werden. 

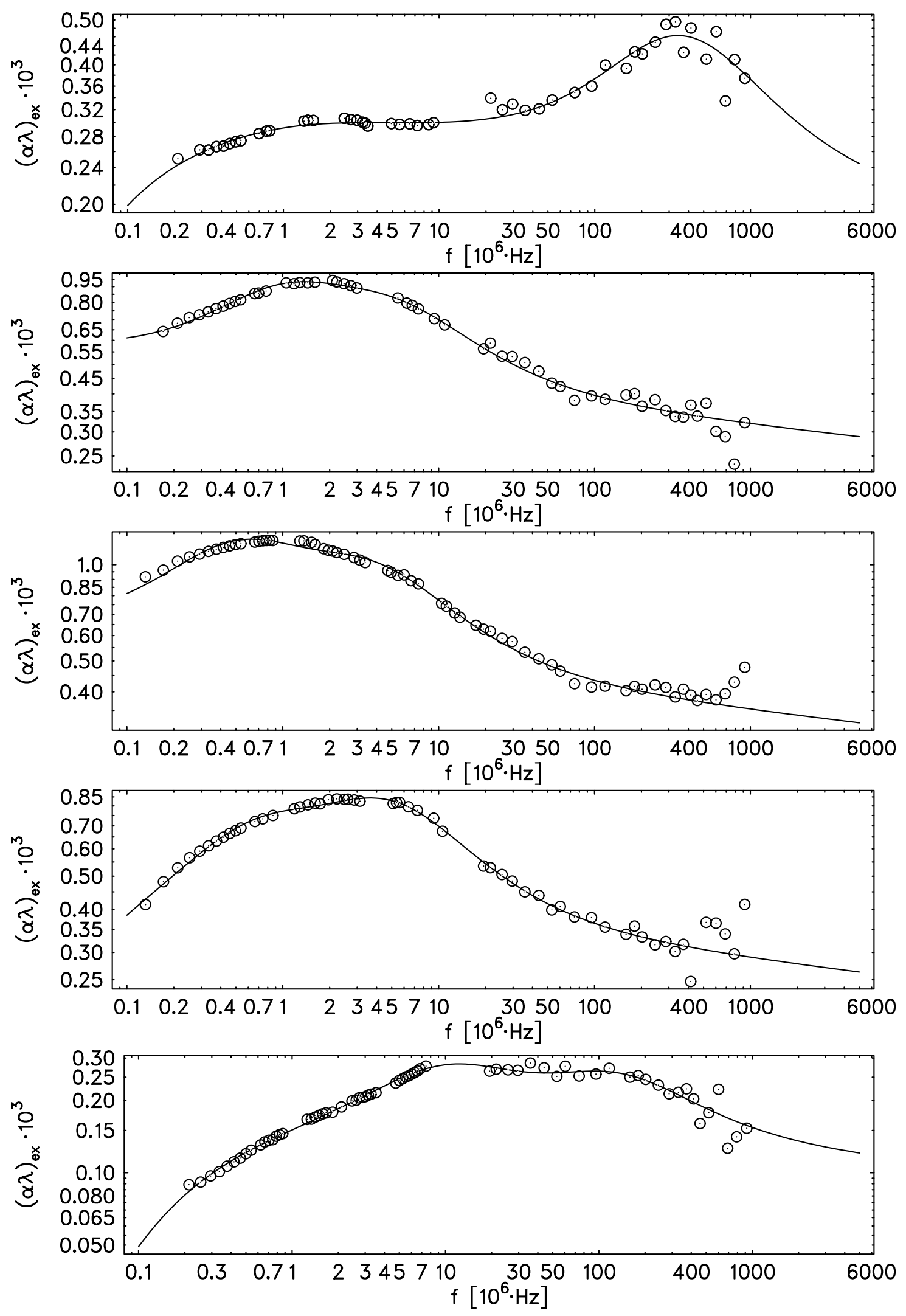

Abb. 7.13: Ultraschallspektren der DMPC/DPPC 83:17 $2 \frac{\mathrm{mg}}{\mathrm{ml}}$ Suspensionen; Temperatur von oben nach unten: $23{ }^{\circ} \mathrm{C}, 26{ }^{\circ} \mathrm{C}, 27{ }^{\circ} \mathrm{C}, 28$ ${ }^{\circ} \mathrm{C}, 31{ }^{\circ} \mathrm{C}$ mit angepaßter Spektralfunktion (7.2) 

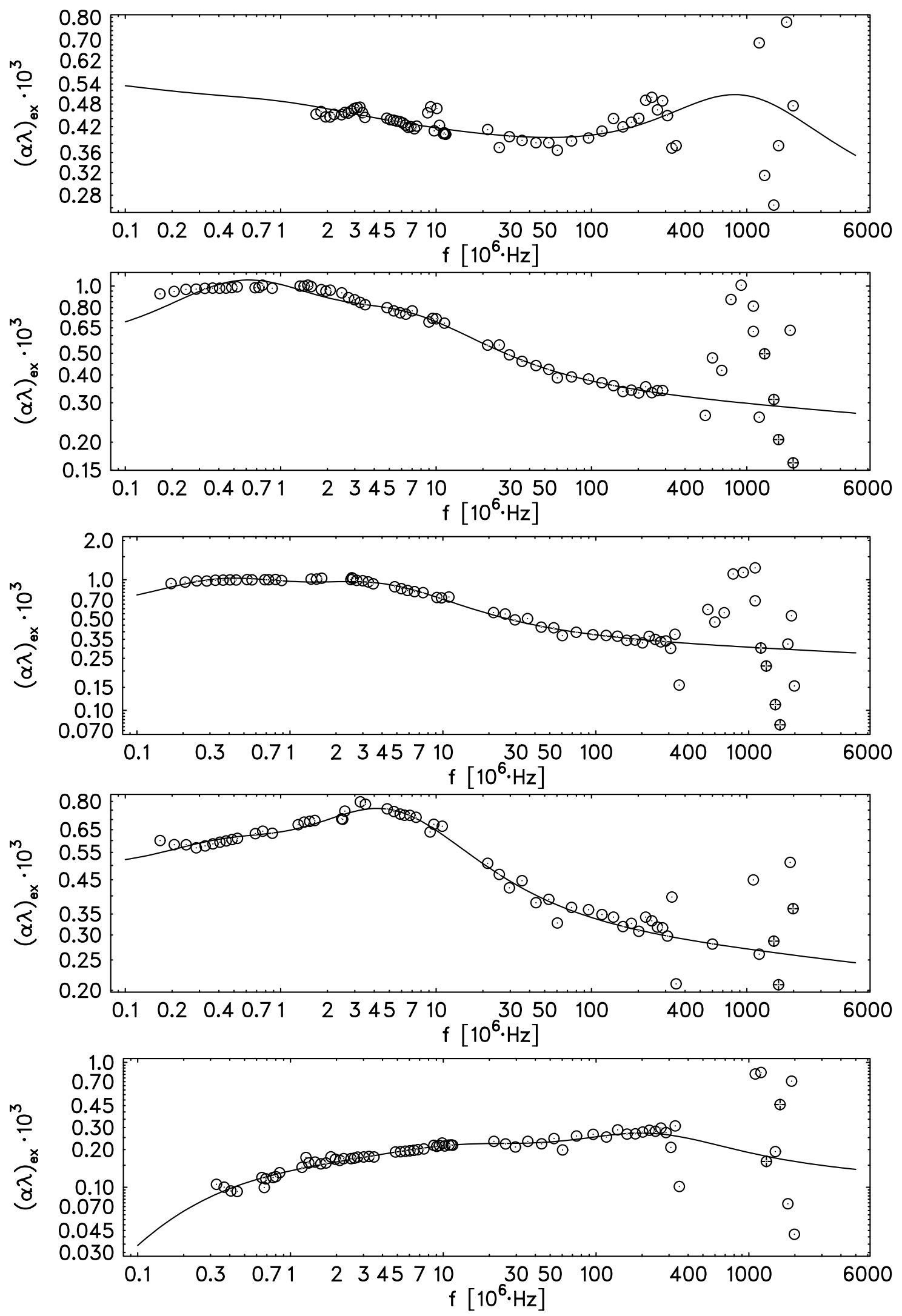

Abb. 7.14: Ultraschallspektren der DMPC/DPPC 50:50 $2 \frac{\mathrm{mg}}{\mathrm{ml}}$ Suspensionen; Temperatur von oben nach unten: $29{ }^{\circ} \mathrm{C}, 32{ }^{\circ} \mathrm{C}, 33^{\circ} \mathrm{C}, 34$ ${ }^{\circ} \mathrm{C}, 37{ }^{\circ} \mathrm{C}$ mit angepaßter Spektralfunktion (7.2) 

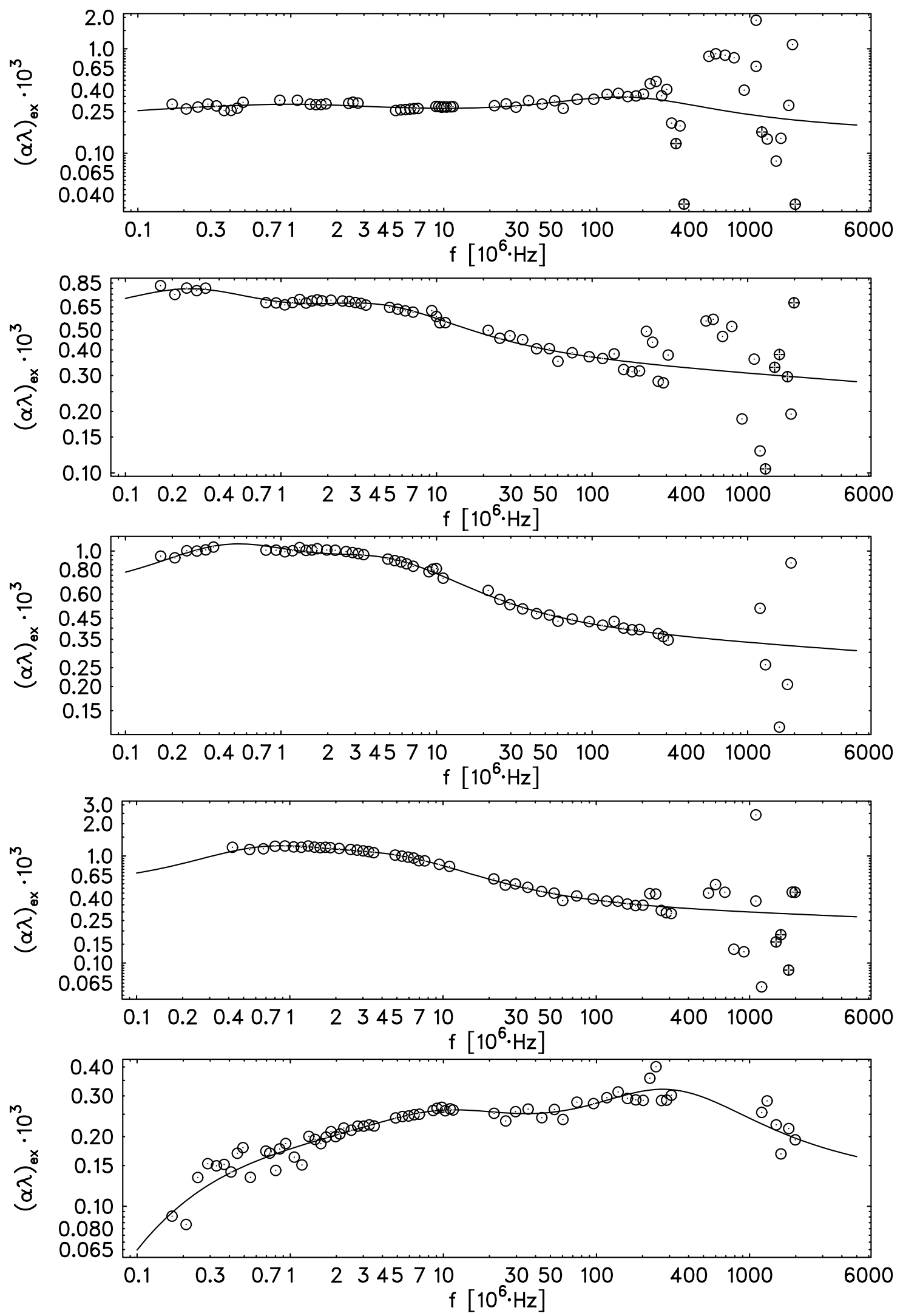

Abb. 7.15: Ultraschallspektren der DMPC/DPPC 17:83 $2 \frac{\mathrm{mg}}{\mathrm{ml}}$ Suspensionen; Temperatur von oben nach unten: $34^{\circ} \mathrm{C}, 37^{\circ} \mathrm{C}, 38^{\circ} \mathrm{C}, 39$ ${ }^{\circ} \mathrm{C}, 42{ }^{\circ} \mathrm{C}$ mit angepaßter Spektralfunktion (7.2) 


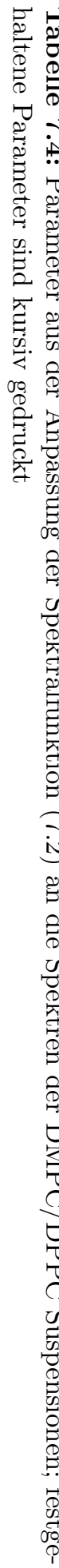

\begin{tabular}{|c|c|c|c|c|c|c|c|}
\hline 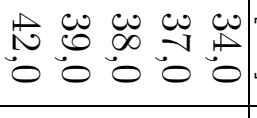 & $\stackrel{\circ}{2} \mapsto$ & & 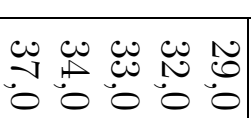 & $\vec{\Omega} \cdot$ & & 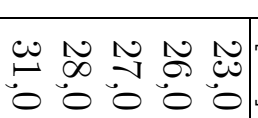 & $\stackrel{\circ}{2}-1$ \\
\hline$\sim \sim \sim \sim N$ & 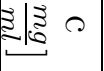 & & $\sim \sim N \sim N$ & 赵苟 & & $\sim \sim \sim \sim \sim$ & 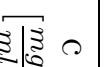 \\
\hline 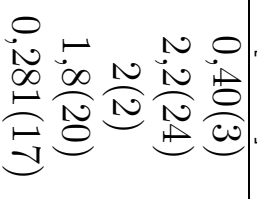 & & & 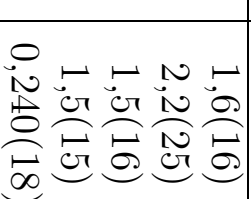 & 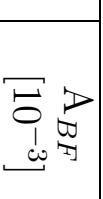 & & 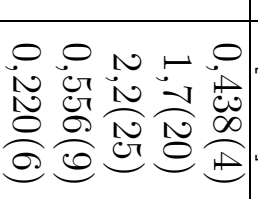 & 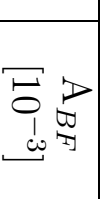 \\
\hline 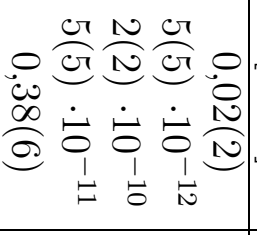 & 窯 & & 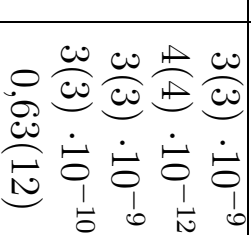 & 胥 & & 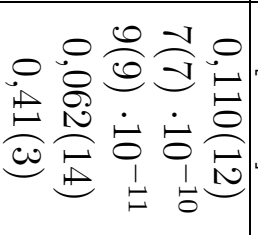 & 茎り \\
\hline 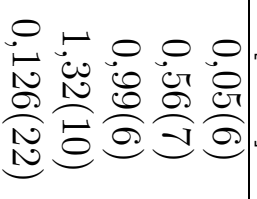 & 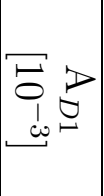 & 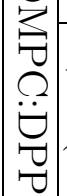 & 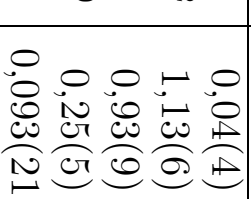 & 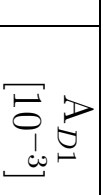 & 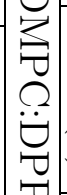 & 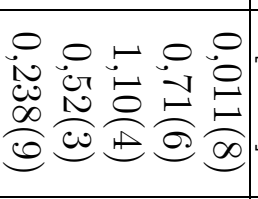 & 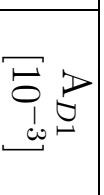 \\
\hline 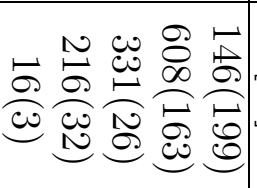 & $\vec{v}_{0}^{-1}$ & $\left|\begin{array}{l}\overrightarrow{1} \\
0 \\
0 \\
0 \\
0\end{array}\right|$ & 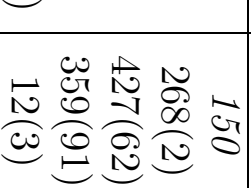 & $\vec{\theta}, \vec{\theta}$ & 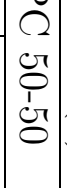 & 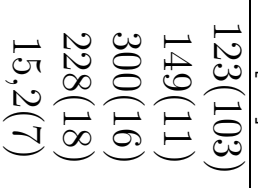 & $\overrightarrow{E_{0}} \theta^{-1}$ \\
\hline 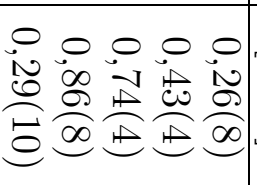 & 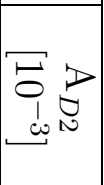 & & 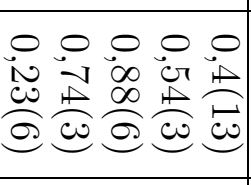 & 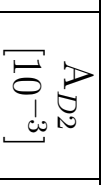 & & 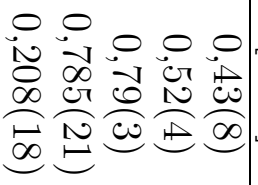 & 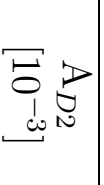 \\
\hline 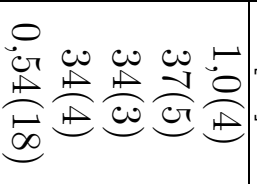 & 苞总 & & 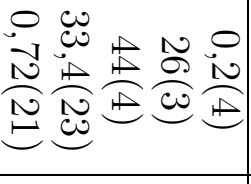 & 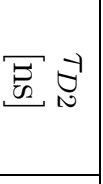 & & 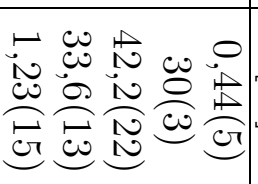 & 萬总 \\
\hline 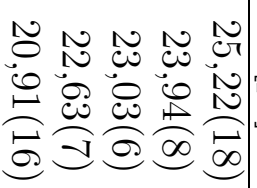 & $\bar{z}_{0}$ & & 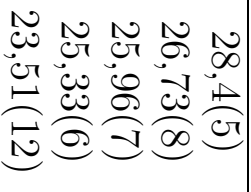 & 总 & & 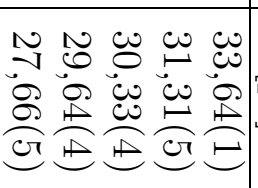 & $\nabla_{\infty} \varpi$ \\
\hline
\end{tabular}




\subsection{Der Fluktuations-Term}

In allen gemessenen Spektren dominiert über weite Frequenzbereiche, insbesondere zu tiefen Frequenzen hin, der die Fluktuationen beschreibende BhattacharjeeFerrell-Term. In der Nähe der Hauptumwandlungstemperatur ist das System durch ausgeprägte Dichtefluktuationen gekennzeichnet, bei dem in der Membran dichte und gelförmige Bereiche neben den weniger dichten fluiden Bereichen existieren. Diese Bereiche bilden Domänen die nebeneinander koexistieren und fortwährend ihre Größe, Form und Position auf der Membran ändern, was zu ständigen Dichteänderungen der Membran führt.

\subsubsection{Die Debye-Terme unter der Betrachtung verschiedener Skalierungsfunktionen}

In der Diskussion um die bestmögliche Skalierungsfunktion, ergibt sich die Frage, ob die bisher angepaßten Debye-Terme nicht nur Korrekturen einer unzureichenden Theorie sind. Die Bhattacharjee-Ferrell-Theorie sollte Dichtefluktuationen auf allen Skalen beinhalten also auch mögliche Einzelmolekülprozesses wie Kinkenbildungen der Ketten (siehe Kapitel 7.5). Da dieses aber in der Realität oder zumindest bei den hier gemessenen Spektren nicht der Fall ist, liegt die Vermutung nahe, daß DebyeTerme nur zur Korrektur der Theorie dienen, aber keinen physikalischen Hintergrund besitzen. Sollte dieses der Fall sein, so müßte sich bei geeigneter Wahl der Skalierungsfunktion die Stellung der Debye-Terme im Spektrum merklich verändern oder die Debye-Terme könnten sogar völlig verschwinden. Aus diesem Grund werden versuchsweise auf ein geeignetes Spektrum alle oben in Kapitel 5.4 und 5.5 beschriebenen Modelle für kritisches Verhalten angepaßt. Für diesen Test wird das Spektrum der DMPC Suspension $10 \frac{\mathrm{mg}}{\mathrm{ml}}$ bei $28^{\circ} \mathrm{C}$ augewählt, da sich dieses Spektrum für die folgende Analyse besonders eignet, da die Debye-Terme gut getrennt voneinander im Frequenzspektrum liegen. Somit sollten mögliche Unterschiede bei den DebyeTermen während der Anpassung der unterschiedlichen Spektralfunktionen gut zu erkennen sein.

\begin{tabular}{|c|c|c|c|c|c|}
\hline Modell & $A_{1}$ & $\tau_{1}[\mathrm{~ns}]$ & $A_{2}$ & $\tau_{2}[\mathrm{~ns}]$ & $\mathrm{B}[\mathrm{ps}]$ \\
\hline Fixman-Kawasaki & $1,64(79)$ & $4,84(21)$ & $2,15(68)$ & $0,237(58)$ & $31,31(46)$ \\
Kroll-Ruhland & $1,27(11)$ & $5,82(34)$ & $2,844(13)$ & $0,12(11)$ & $30,5(20)$ \\
B.-F.1985a & $1,262(84)$ & $5,69(19)$ & $2,8(36)$ & $0,122(84)$ & $30,5(15)$ \\
B.-F.1985b & $1,260(84)$ & $5,71(19)$ & $2,8(37)$ & $0,121(85)$ & $30,5(15)$ \\
B.-F.1997 & $1,508(67)$ & $5,54(20)$ & $2,1(13)$ & $0,178(71)$ & $30,93(70)$ \\
\hline
\end{tabular}

Tabelle 7.5: Parameter der zwei Debye-Terme aus den Anpassungen der unterschiedlichen Fluktuationsmodelle

Es zeigt sich, daß sich mittels der Terme aus verschiedenen Fluktuationstheorien und zweier Debye-Termen das Spektrum immer hinreichend beschreiben läßt. Allerdings sind deutliche Unterschiede in der Qualität der Anpassung zu entdecken. Daß sich die zwei Debye-Terme weder im Bezug auf Relaxationszeit noch Relaxationsamplitude verändern, geschweige denn ganz verschwinden (siehe Tabelle 7.5) ist als wichtigstes Ergebnis dieser Berechnung ist anzusehen. 
Dieses läßt darauf schließen, daß die Debye-Prozesse doch reale molekulare Ursachen haben, die nicht in den Fluktuationsmodellen enthalten sind.

\subsubsection{Die Skalierungsfunktion}

Wegen der Unsicherheit in der Bestimmung der am besten geeigneten Skalierungsfunktion, wird nun diese Funktion selbst gemessen, um die Übereinstimmung von Theorie und Meßdaten zu überprüfen und um eine weitere Legitimation für die Anwendbarkeit der Bhattacharjee-Ferrell-Theorie zu bekommen. Bei hohen Frequenzen wird der kritische Beitrag zum Ultraschallspektrum weitestgehend von dem Hintergrundanteil $B \cdot f$ und von den zusätzlichen Einzelzeitrelaxationen dominiert. Das ist bei sehr tiefen Frequenzen anders: Unterhalb von ca. 1,8 MHz wird der kritische Beitrag derart stark, daß er alle anderen Effekte fast überdeckt. Dennoch ist selbst dort eine merkliche Einwirkung der Debye-Terme zum Ultraschallspektrum zu registrieren.

Es wurde nun eine DMPC/Wasser Suspension $2 \frac{m g}{m l}$ angesetzt und deren Ultraschalldämpfung bei verschiedenen Temperaturen um die Umwandlungstemperatur ermittelt. Da für das Messen der Skalierungsfunktion nur die tieffrequenten Punkte interessant sind, diente als Meßapparatur dazu der $1 \mathrm{MHz}$ Resonator. Von den gemessenen $\alpha / f^{2}$-Werte wurden anschließend die nichtkritischen Beiträge abgezogen, wie sie aus den breitbandigen Ultraschallspektren bekannt waren, um so den kritischen Beitrag $(\alpha \lambda)_{c}$ zur Ultraschalldämpfung zu erhalten. Es zeigt sich, daß erst das Herausrechnen der zusätzlichen Beträge, die durch die Debye-Terme verursacht sind, die Meßpunkte in die Nähe des Verlaufs der Skalierungsfunktion bringt. Durch bewußte, allerdings im Rahmen der Fehler, wie sie aus den Anpaßrechnungen der Ultraschallspektren von $200 \mathrm{kHz}$ bis $2 \mathrm{GHz}$ bekannt sind, Modifizierung der Parameter der Debye-Terme und des B-Wertes kann eine sehr gute Übereinstimmung der gemessenen Punkte mit der entsprechenden Skalierungsfunktion gefunden werden.

Mittels der drei bekannten empirischen Skalierungsfunktionen der BhattacharjeeFerrell-Theorie können so drei explizite Verläufe derselben Skalierungsfunktionen errechnet werden. Abb. 7.16 und Abb. 7.17 zeigen exemplarisch den Verlauf von $(\alpha \lambda)(T) /(\alpha \lambda)_{c}\left(T_{c}\right)=F(\Omega)$ anhand der Skalierungsfunktion (5.28) einmal unterhalb und einmal oberhalb der Umwandlungstemperatur.

Wie man sieht, streuen die Punkte teilweise recht stark um die analytische Funktion. Auffällig ist vor allem, daß bei den relativ tiefen Temperaturen die Punkte zum Teil eine charakteristische Abweichung aufweisen. Dieser Verlauf der Meßpunkte liegt auch bei der Auswertung der Daten mittels der anderen beiden Skalierungsfunktionen vor, so daß abschließend festgestellt werden muß, daß eine klare Entscheidung, welche Skalierungsfunktion nun die "richtige" ist, im Rahmen der hier vorliegenden Meßgenauigkeit nicht getroffen werden kann. Insbesondere weit außerhalb der Umwandlung zeigen sich charakteristische Abweichungen von der analytischen Funktion.

Es zeigt sich jedoch, daß die Berechnung der jeweiligen Abweichung der gemessenen Punkte von der analytischen Skalierungsfunktion

$$
\chi=\frac{1}{N} \sum_{i=1}^{N}\left(\frac{(\alpha \lambda)(T)}{(\alpha \lambda)_{c}\left(T_{c}\right)}-F(\Omega)\right)^{2}
$$




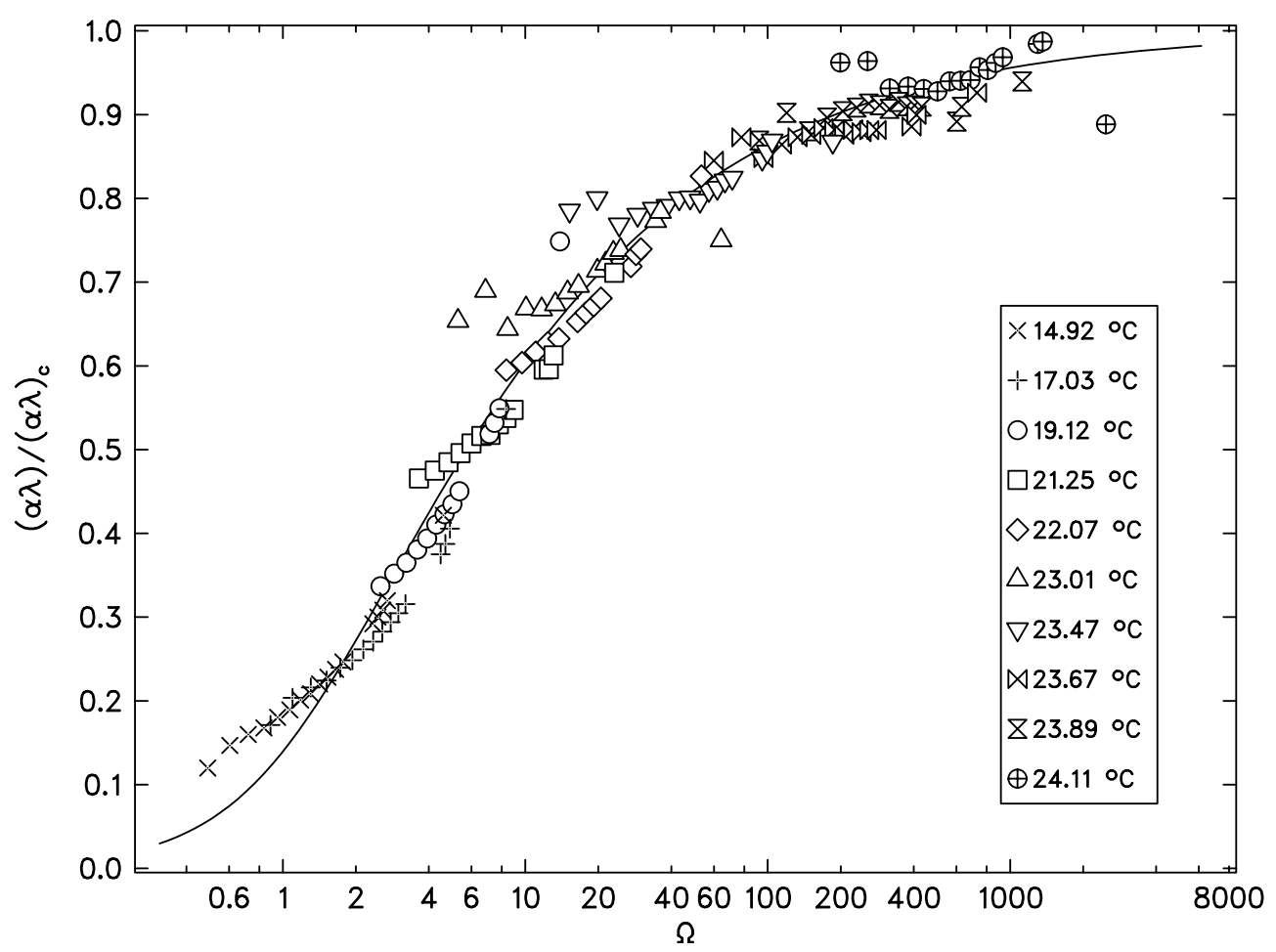

Abb. 7.16: Meßkurve von $\alpha \lambda$ bei $T$ zu $\alpha \lambda$ bei $T_{c}$ einer DMPC $2 \frac{m g}{m l}$ Lösung unterhalb von $T_{c}$. Die Kurve ist der Graph der Skalierungsfunktion (5.28).

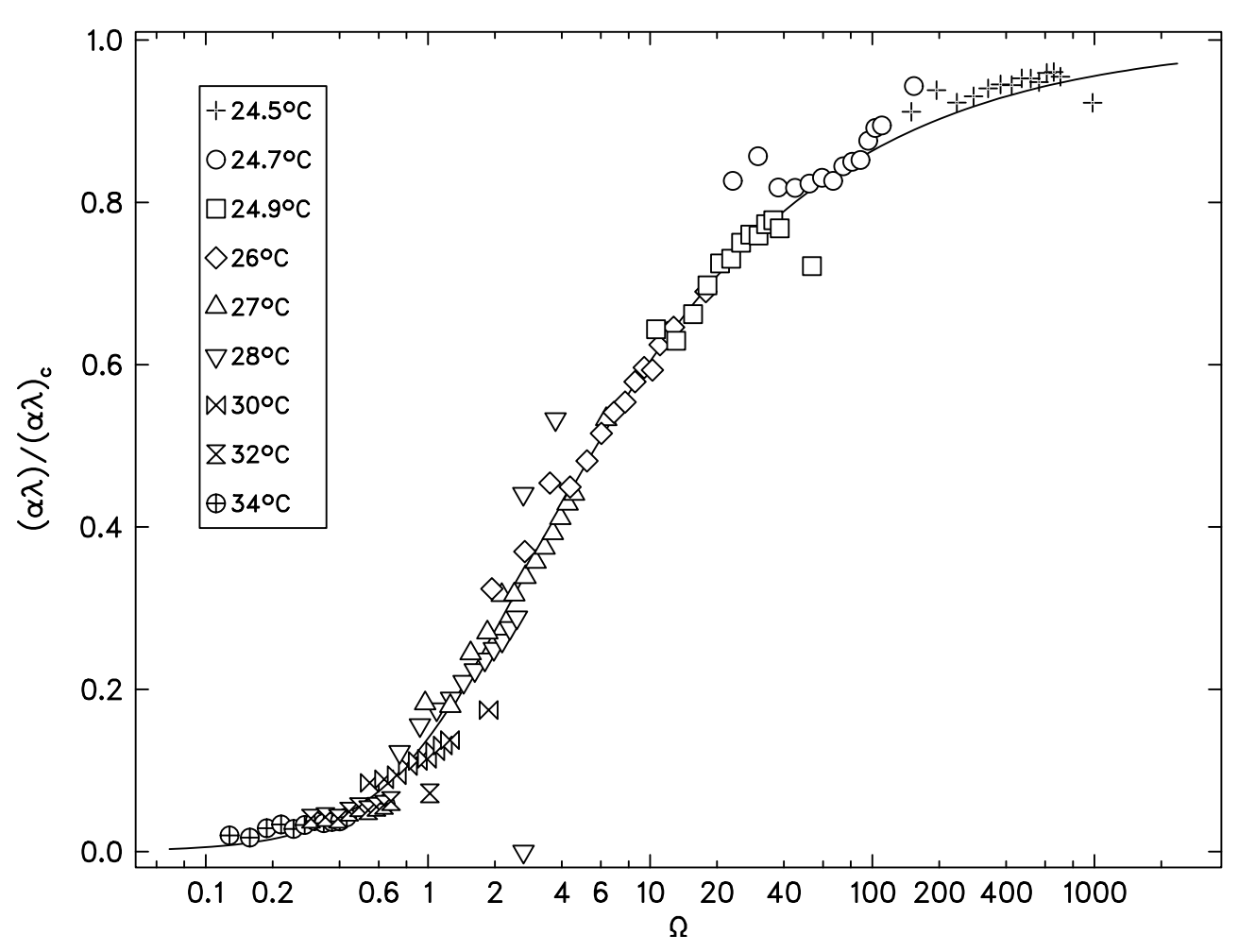

Abb. 7.17: Meßkurve von $\alpha \lambda$ bei $T$ zu $\alpha \lambda$ bei $T_{c}$ einer DMPC $2 \frac{m g}{m l}$ Lösung oberhalb von $T_{c}$. Die Kurve ist der Graph der Skalierungsfunktion (5.28). 
eine - allerdings nur geringfügig - bessere, Übereinstimmung der Punkte mit der in Abb. 7.16 und Abb. 7.17 dargestellten Skalierungsfunktion (5.28) bringt.

\begin{tabular}{|c|c|c|c|}
\hline & B.-F.1997 & B.-F.1985b & B.-F.1985a \\
\hline$T<T_{c}$ & $1,47 \cdot 10^{-3}$ & $1,45 \cdot 10^{-3}$ & $1,61 \cdot 10^{-3}$ \\
$T>T_{c}$ & $1,88 \cdot 10^{-3}$ & $2,10 \cdot 10^{-3}$ & $2,15 \cdot 10^{-3}$ \\
\hline
\end{tabular}

Tabelle 7.6: Abweichung $\chi$ der Meßpunkte von den Skalierungsfunktionen

\subsubsection{Kritische Exponenten}

Der Temperaturgang der charakteristischen Relaxationsrate $\omega_{B F}$ läßt sich mit Hilfe der folgenden Gleichung

$$
\omega_{B F}(t)=\frac{2 D}{\xi^{2}}=\bar{\gamma}_{0}\left|T-T_{c}\right|^{z \nu}
$$

mittels eines Potenzgesetzes beschreiben. Dabei ist $z=3,05$ der Dynamikexponent und $\nu=0,63$ der kritische Exponent der Korrelationslänge. Es gilt somit für den gesamten Exponenten in Gleichung (7.5) $z \nu=3,05 \cdot 0,63=1,9215$. Im Gegensatz zu den sonst "üblichen" kritischen Systemen, bei denen beispielsweise bei $T=T_{c}$ Entmischung auftritt, ist es bei den Membransystemen möglich, von beiden Seiten, also sowohl oberhalb als auch unterhalb von $T_{c}$, den kritischen Exponenten zu bestimmen.

Mittels der temperaturabhängigen, charakteristischen Relaxationsrate $\omega_{B F}(t)$, wie sie aus den Messungen zur Skalierungsfunktion bestimmt werden kann, werden nun unter Vorgabe der Umwandlungstemperatur $T_{c}=24,3^{\circ} \mathrm{C}$ der kritische Exponent $z \nu$ und die Konstante $\bar{\gamma}_{0}$ mit Hilfe einer Anpaßrechnung der Gleichung (7.5) errechnet.

\begin{tabular}{|c|c|c|}
\hline & $\overline{\gamma_{0}\left[\frac{\mathrm{Hz}}{{ }^{\circ} \mathrm{C}}\right]}$ & $z \nu$ \\
\hline$T<T_{c}$ & $6696(1752)$ & $1.73(13)$ \\
$T>T_{c}$ & $16401(1356)$ & $1.923(38)$ \\
\hline
\end{tabular}

Tabelle 7.7: Parameter aus der Anpassung von Gleichung (7.5) in Abb. 7.18

In Tabelle 7.7 sind die Werte aus der Anpassung mit den zugehörigen Fehler aufgeführt. Bemerkenswert dabei ist die Tatsache, daß der berechnete kritische Exponent aus den Meßdaten klar im Bereich des theoretischen Wertes liegt. Dieses scheint auf den ersten Blick nicht weiter verwunderlich, da unter Voraussetzung der Lage der gemessenen Punkte auf der Skalierungsfunktion die Annahme nahe liegt, sie folgen auch den theoretischen Potenzgesetzen. Es besteht jedoch entgegen dieser Überlegungen die Möglichkeit einer Unstimmigkeit im Verhältnis von $\omega_{B F}(t) \mathrm{zu}$ $A_{B F}(t)$ der einzelnen Messungen. Dies scheint aber offensichtlich nicht der Fall zu sein, da $z \nu$ in der Nähe des theoretischen Wertes liegt. 


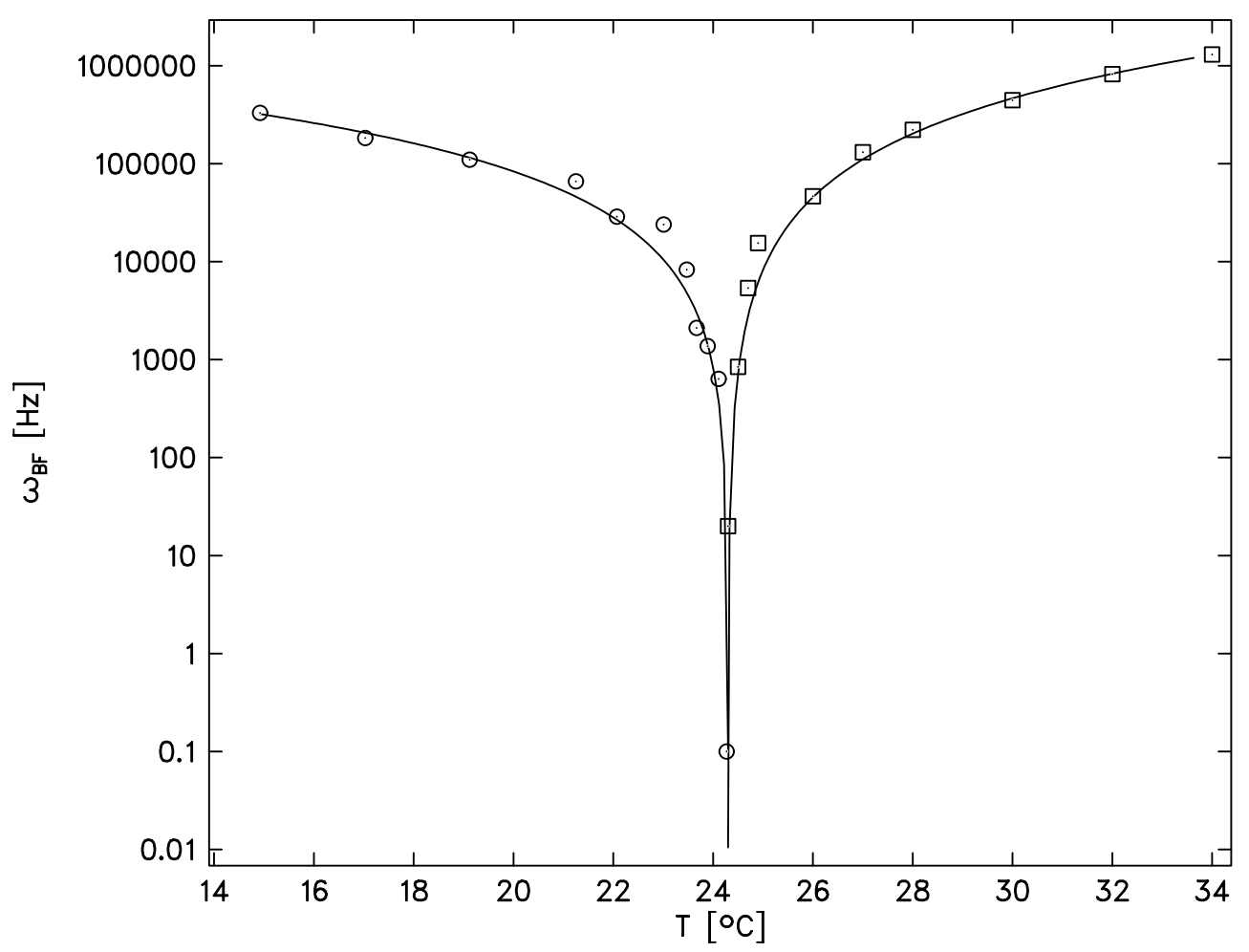

Abb. 7.18: Anpassung von Gleichung (7.5) an die Werte $\omega_{B F}(t)$ aus der Messung der Skalierungsfunktion unter Festhalten des Parameters $T_{c}=24,3^{\circ} \mathrm{C}$

\subsubsection{Verhalten am kritischen Punkt}

Ausgehend von Gleichung (5.29) gilt im folgenden

$$
\begin{aligned}
(\alpha \lambda) & =A_{B F}(T) F(\Omega) f^{-0,057} \\
\left(\frac{\alpha}{f^{2}}\right) & =\frac{A_{B F}(T)}{c_{s}} F(\Omega) f^{-1,057} .
\end{aligned}
$$

Nimmt man nun an, die Temperatur läge in der Nähe des kritischen Punktes, dann gilt wegen $\Omega=\omega / \omega_{B F}$ und $\omega_{B F} \approx 0$

$$
\Omega \approx \infty
$$

D.h. allerdings für $F(\Omega \Rightarrow \infty) \approx 1$ (siehe auch Abb. 7.16). Der Verlauf der Ultraschalldämpfung über der Frequenz läßt sich somit am kritischen Punkt nach Abzug des klassischen Hintergrundanteils durch folgende einfache Gleichung beschreiben:

$$
\left(\frac{\alpha}{f^{2}}\right)=\frac{A_{B F}(T)}{c_{s}} f^{-1,057} .
$$

Zieht man folglich von dem vorhandenen Spektrum den Anteil der Debye-Terme und den Hintergrundanteil ab und trägt $\left(\frac{\alpha}{f^{2}}\right)$ gegen $f^{-1,057}$ auf, so liegen alle Meßpunkte auf einer Geraden. Das Abziehen der nichtkritischen Anteile ist dabei evident.

Abb. 7.19 zeigt dieses für die DMPC $10 \frac{\mathrm{mg}}{\mathrm{ml}}$ Suspension bei $24^{\circ} \mathrm{C}$. Alle Punkte liegen ziemlich genau auf einer Geraden. Die Steigung der Geraden beträgt hierbei 


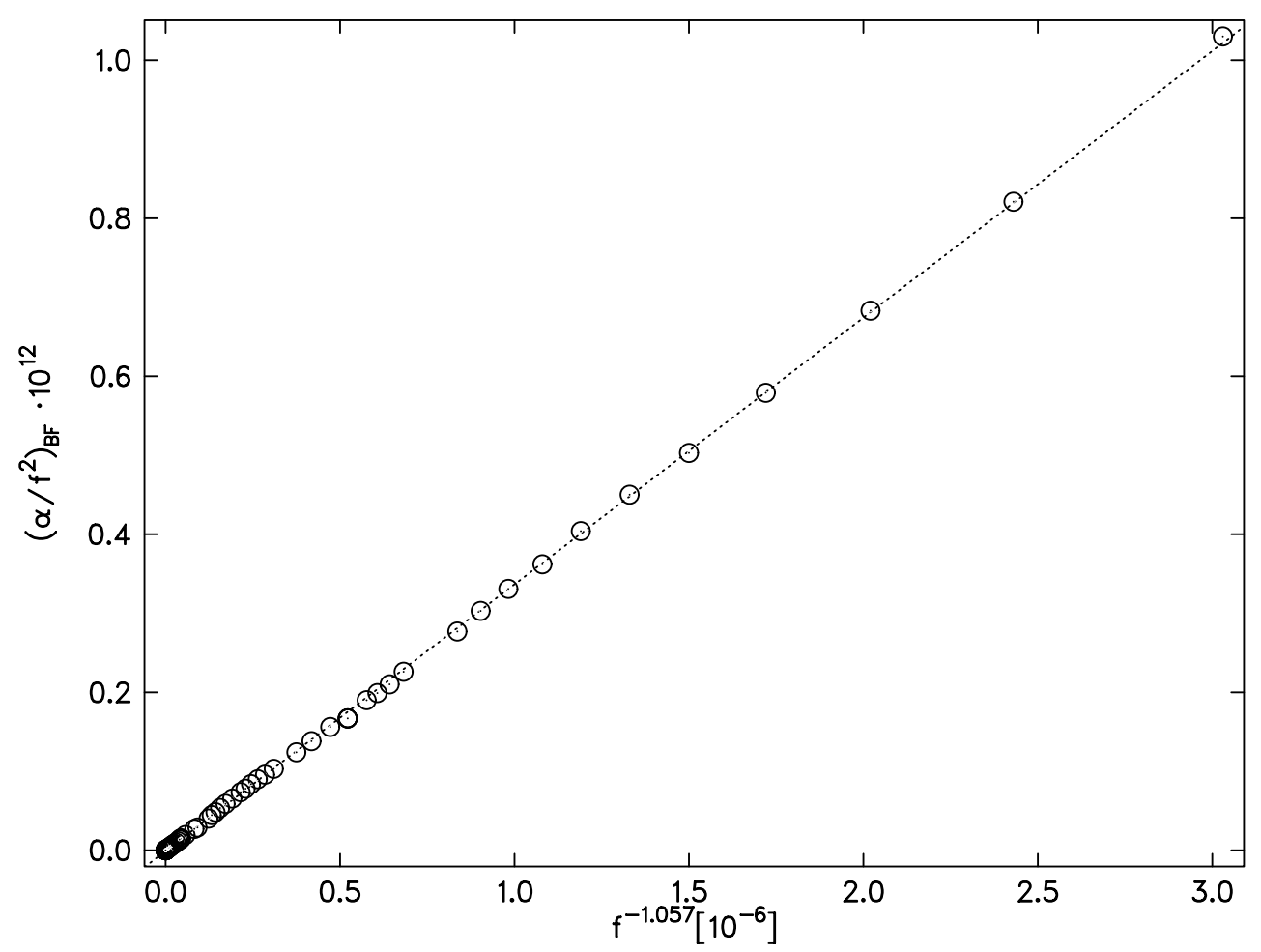

Abb. 7.19: $\frac{\alpha_{e x}}{f^{2}}$ gegen $f^{-1,057}$ der DMPC $10 \frac{m g}{m l}$ Suspension bei $24^{\circ} \mathrm{C}$ nach Abzug der beiden Debye-Terme und des Hintergrundanteils

$\frac{A_{B F}(T)}{c_{s}}=3.5 \frac{\mu s}{m}$. Berechnet man die Steigung aus der Amplitude der BhattacharjeeFerrell-Funktion bei $24^{\circ} \mathrm{C}$ so ergibt sich $\frac{A_{B F}(T)}{c_{s}}=5.4 \frac{\mu s}{m}$, was in Anbetracht des sehr hohen Fehlers der Amplitude der Bhattacharjee-Ferrell-Funktion, der weit über 100 $\%$ liegt, als gute Übereinstimmung gewertet werden muß.

\subsubsection{DMPC / Wasser}

Es wurden insgesamt Suspensionen zweier unterschiedlicher Konzentrationen des DMPC / Wasser Systems gemessen: zum einen $2 \frac{\mathrm{mg}}{\mathrm{ml}}$ und zum anderen $10 \frac{\mathrm{mg}}{\mathrm{ml}}$. Nach Halstenberg et al. [49] ist die auftretende Ultraschalldämpfung bei geringen Lipidkonzentrationen linear mit der Konzentration. Für die hier getätigten Messungen besteht somit die Möglichkeit der Normierungs der auftretenden Amplituden auf die Konzentration und folglich der direkten Vergleichbarkeit miteinander. Die durch die Anpaßrechnungen ermittelten Relaxationszeiten und Frequenzen können allerdings wie ermittelt belassen werden. In Abb. 7.20 ist die normierte Amplitude des Bhattacharjee-Ferrell-Terms über der Temperatur dargestellt. Deutlich ist ein Anstieg dieses Parameters zur kritischen Temperatur bei $T_{c} \approx 24^{\circ} \mathrm{C}$ zu sehen. Ausgehend von den Werten weit außerhalb der Umwandlungstemperatur steigt die Bhattacharjee-Ferrell-Amplitude um das zehn- bis zwanzigfache bei $T_{c}$ an, was eindeutig das kritische Verhalten des DMPC / Wasser Systems charakterisiert. Im Bild der fluktuierenden Membran bedeutet dieses zur Umwandlungstemperatur mehr und mehr zunehmende Fluktuationen, die der Ultraschallwelle immer mehr Energie entziehen. Mehr und größere Bereiche der Membran nehmen an den Fluktuationen Teil. Die Amplitude geht in dem hier gemessenen System allerdings nicht gegen $\infty$, da die 


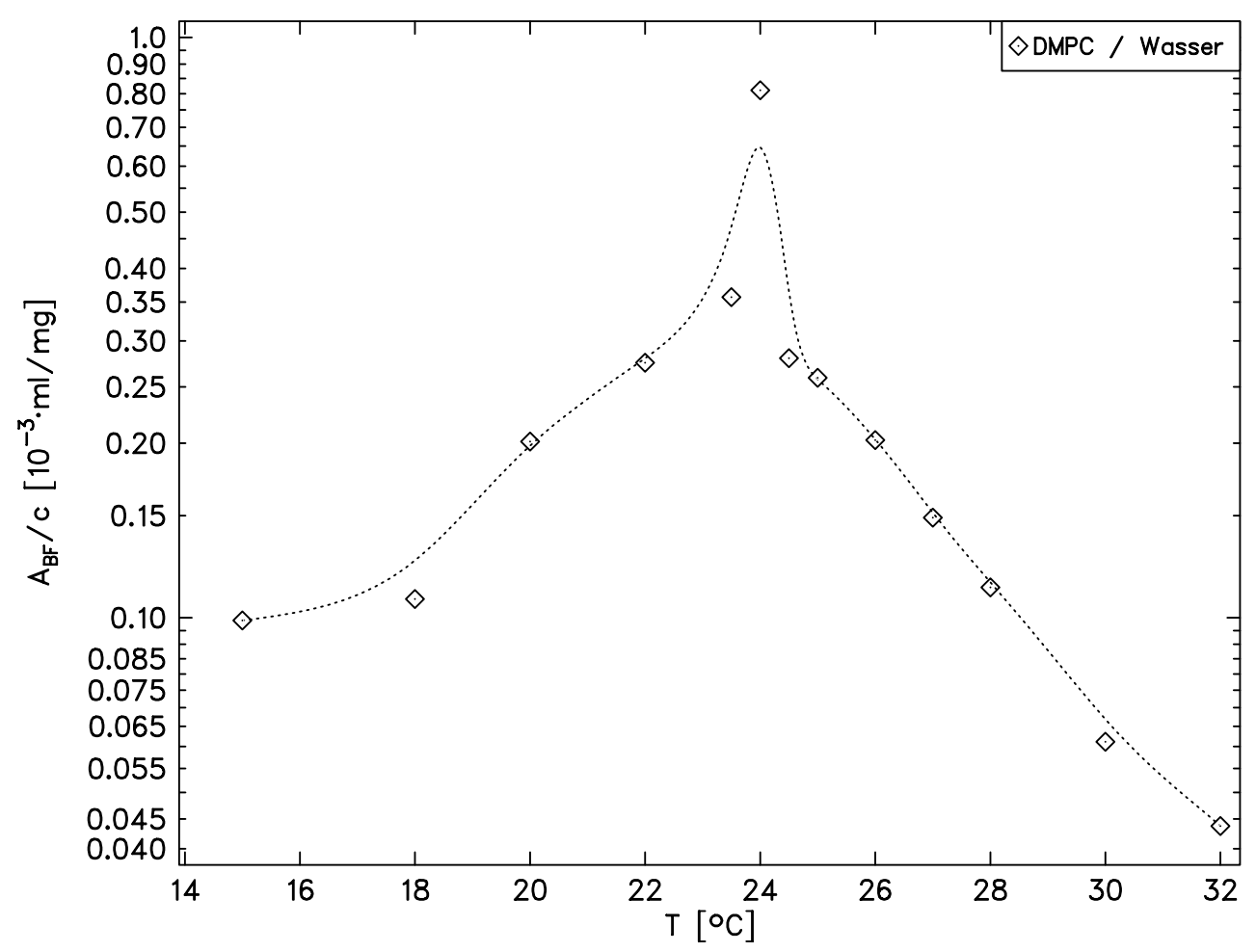

Abb. 7.20: Normierte Amplitude der BF-Spektralfunktion von den DMPC / Wasser Suspensionen

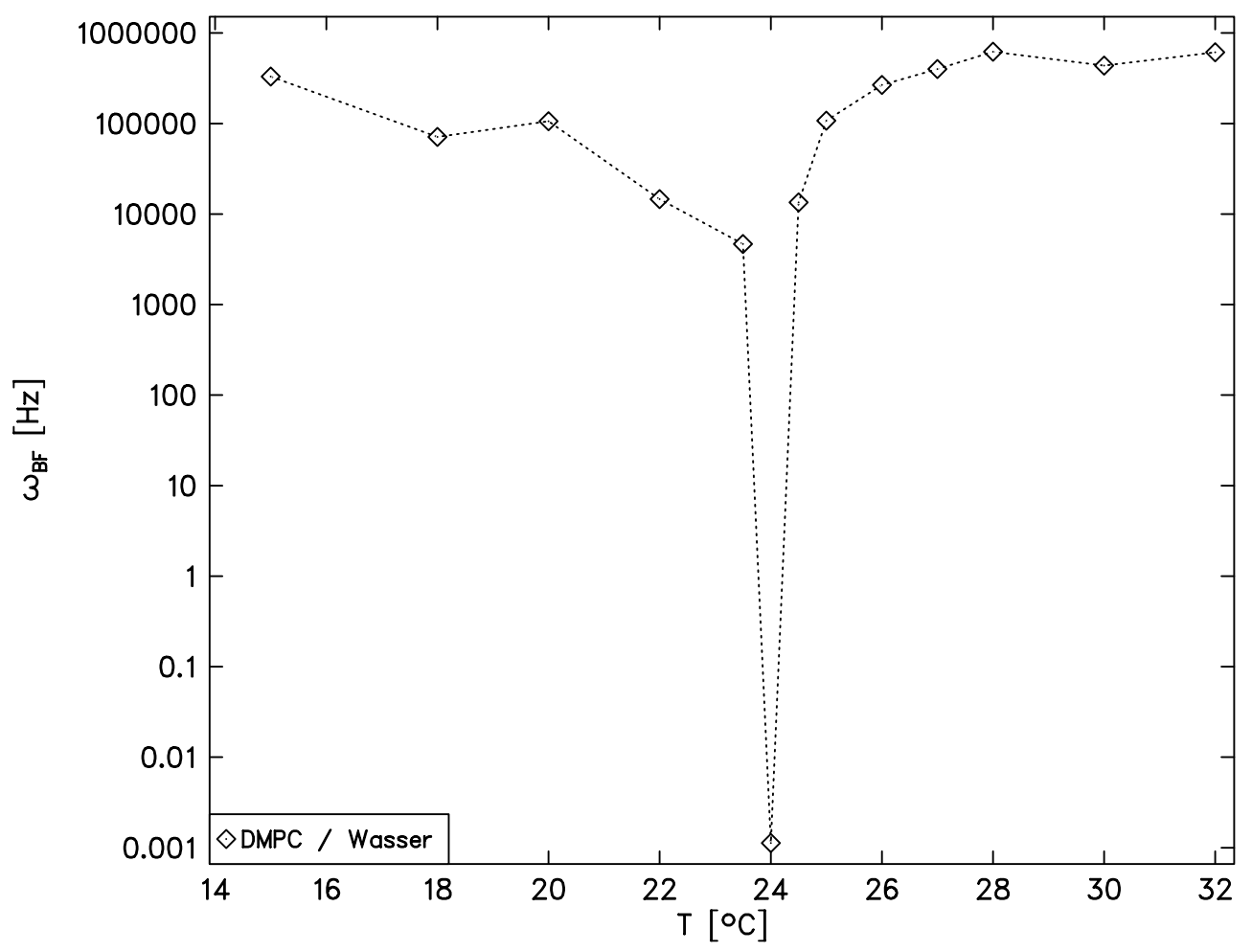

Abb. 7.21: Charakteristischen Frequenz $\omega_{B F}$ der BF-Spektralfunktion von den DMPC / Wasser Suspensionen 
Konzentration des Lipids in der Wasserlösung doch relativ gering ist, und vergleichsweise große Bereiche der Lösung aus reinem, keine Zusatzdämpfung verursachendem Wasser bestehen. Alternativ zur Amplitude ist in Abb. 7.21 die charakteristische Bhattacharjee-Ferrell-Relaxationsrate $\omega_{B F}$ über der Temperatur dargestellt. Analog zur Amplitude zeigt sich hier ein starkes Absinken der Rate bei $T_{c}$. Dieses erstreckt sich bei der Rate über mehrere Dekaden. Die zur Umwandlungstemperatur immer größer werdenden Bereiche fluktuieren immer langsamer und die Domänen werden zunehmend langlebiger. Die Bhattacharjee-Ferrell-Rate geht bei $T_{c}$ gegen Null, da sich diese Größe allein auf die Geschwindigkeit der Fluktuationen der Domänen auf den Vesikeln bezieht und in keinem direkten Zusammenhang zu der Lipid/Wasser Konzentration steht.

\subsubsection{Die Korrelationslänge}

Die Korrelationslänge ist ein Maß für die Reichweite von Wechselwirkungen. Sie gibt an, in welcher Entfernung von einem bestimmten Punkt noch Veränderungen der physikalischen Eigenschaften spürbar sind und Auswirkungen haben. In der Theorie von Bhattacharjee und Ferrell ist die charakteristische Relaxationsrate der Fluktuationen $\omega_{B F}$ mit der Korrelationslänge verknüpft:

$$
\omega_{B F}(T)=\frac{2 D}{\xi^{2}}=\frac{k_{B} T}{3 \pi \eta \xi^{3}}=\bar{\gamma}_{0}\left(T-T_{c}\right)^{z \nu} .
$$

Außerdem läßt sich der Gang der Korrelationslänge mittels eines Potenzgesetzes beschreiben:

$$
\xi=\xi_{0} \cdot\left(\frac{T-T_{c}}{T_{c}}\right)^{-\nu} .
$$

Somit gilt bei $T=T_{c} \Rightarrow \xi \approx \infty$ ! Um nun die Korrelationslänge der in dieser Arbeit betrachteten Systeme auszurechnen, werden entweder Informationen über den Diffusionskoeffizienten $D$ oder der Viskosität $\eta$ benötigt. Dabei handelt es sich hierbei nicht um die sonst üblichen dreidimensionalen Diffusionskoeffizienten bzw. Viskositäten, sondern, da Bewegungen in der Membran nur lateral möglich sind, um die zweidimensionalen Entsprechungen der jeweiligen Größen. Die Viskosität wäre z.B. nicht über die auftretende Schubspannung an zwei lamminar aneinander vorbei strömenden Schichten innerhalb einer Flüssigkeit definiert sondern hier über die Spannung zweier Bereiche auf der Membran, die sich aneinander vorbei bewegen. Analog geht man bei der Diffusion vor. Man schafft ein Ungleichgewicht von Molekülen eines bestimmten Typs auf der Membran und definiert über die Zeitkonstante der Diffusion dann die zweidimensionale Diffusionskonstante. Dieses wird u.a. bei der sog. FRAP-Methode (Flourescence Recovery After Photobleaching) auf diese Weise gemacht. In die Membran werden dabei Lipide mit Farbstoffsonden eingebracht, die fluoreszierende Eigenschaften besitzen. Mit einem gepulsten Laserstahl wird der Farbstoff einiger Moleküle irreversibel ausgeblichen. Im folgenden wird dann die Diffusion der nicht ausgeblichenen Moleküle in dieser ausgeblichenen Region beobachtet. Bei P. Schwille et al. [132] [90] ist dieses für DLPC/DPPC/Cholesterin Vesikel gemacht worden, und es werden dabei Werte für den Diffusionskoeffizienten in der gelförmigen Phase von $D_{\text {gel }} \approx 2 \cdot 10^{-10} \frac{\mathrm{cm}^{2}}{\mathrm{~s}}$ und in der fluiden Phase von 
$D_{\text {fluid }} \approx 4 \cdot 10^{-8} \frac{\mathrm{cm}^{2}}{\mathrm{~s}}$ angegeben (siehe auch [95] [143] [116]).

\section{Die Natur der Fluktuationen}

Die Ergebnisse der Kaloriemetrie und Velicometrie zeigen zusammenfassend, daß das System in der Nähe der Umwandlung durch starke Dichtefluktuationen bestimmt ist. Es stellt sich jedoch die Frage, in welcher Weise die in Kapitel 7.2.6 in Gleichung (7.10) aufgeführten Diffusionskoeffizienten zu interpretieren sind. Offensichtlich gibt es dafür zunächst zwei Möglichkeiten:

Massediffusion: Die Diffusion der Domänen erfolgt, in dem sich eine relativ feste Konfiguration von $N$ Lipiden eines Zustandes zu einer Domäne mit der Masse $N \cdot M_{\text {Lipid }}$ zusammenfinden, und diese Domäne sich dann mit einer endlichen Geschwindigkeit durch ein Medium bestehend aus Lipiden des anderen Zustandes bewegt. Bedingt wird dieser Vorgang durch die Viskosität des die Domäne umgebenden Mediums bestehend aus Lipiden des jeweils anderen $\mathrm{Zu}$ standes und durch mögliche An- und Ablagerungprozesse an den Domänen, die ebenfalls durch Massediffusion hervorgerufen werden.

Zustandsdiffusion: Jedes Lipid besitzt relativ zur Massediffusion gesehen einen festen Platz auf der Membran. Unterhalb der Hauptumwandlungstemperatur ist diese Anordnung durch ein hexagonales Gitter gekennzeichnet. In der Nähe der Umwandlungstemperatur bilden sich Domänen von gelförmigen und fluiden Bereichen aus, die jeder für sich, bedingt durch die zusätzlich auftretende Hydratationsenergie, bestrebt sind, ihre Randfläche möglichst gering zu halten. Man mittelt nun über die Position aller Lipide einer Domäne und definiert sich so einen Schwerpunkt, der mit der aktuellen Position der Domäne identifiziert werden kann. Durch thermische Stöße wechselt an der einen Seite der Domäne ein Lipid des benachbarten Bereichs seinen Zustand und lagert sich an die Domäne an. Gleichzeitig passiert ähnliches an der anderen Seite der Domäne: Ein Lipid wechselt ebenfalls seinen Zustand, löst sich aus dieser Domäne und muß nun als zu einer anderen Domäne zugehörig angesehen werden. Somit hat sich die Position der Domäne leicht gegenüber der Ausgangssituation verschoben, ohne daß auch nur ein Lipid sich bezogen auf seine Masse bewegt hat. Zusätzlich fluktuiert gleichzeitig das Verhältnis von Molekülen in fluiden Domänen zu Molekülen in gelförmigen Domänen.

Einfache Monte-Carlo-Simulationen [54] von Lipidmembranen realisieren die Zustandsdiffusion und können die Schmelzvorgänge, wie sie sich in den Wärmekapazitätskurven und in den Schallgeschwindigkeitskurven äußern, sehr gut erklären. Weiter wird in Kapitel 7.3 der tieffrequente Debye-Term der Diffusion der Lipide zugeordnet. Der Diffusionsprozeß wird dabei mittels eines Hüpfprozesses über die hexagonale Matrix der Membran beschrieben. So scheint es am wahrscheinlichsten, daß der Fluktuationsbeitrag in den Ultraschallspektren durch die Zustandsdiffusion gekennzeichnet ist.

Die Berechnung der Korrelationslänge, wie sie in Kap. 7.2 .6 angestrebt wird, scheint somit auf den ersten Blick nicht gerechtfertigt. In Gleichung (7.10) geht jedoch die Masse der betrachteten Teilchen nicht ein, so daß die Verwendung dieser Beziehung nach wie vor möglich ist. Jedoch müssen für $D$ nicht die Diffusionskoeffizienten der 
einzelnen Lipidmoleküle verwendet werden sondern vielmehr die Diffusionskoeffizienten der Domänen, deren Werte allerdings nicht bekannt sind. Es zeigt sich jedoch, daß die Verwendung der Diffusionskoeffizienten der einzelnen Lipide bei der Berechnung der Korrelationslänge zu sinnvollen Werten führt, so daß angenommen werden kann, daß die Diffusionskoeffizienten der Domänen zumindest in der gleichen Größenordnung liegen. Es scheint ebenfalls sinnvoll anzunehmen, daß die Diffusionskoeffizienten der Zustandsdiffusion wegen der relativ großen Ausmaße der Domänen unter denen der einzelnen Lipiddiffusion liegen.

\section{Korrelationslänge der Zustandsdiffusion}

Es werden nun Werte für die Berechnung der Korrelationslänge der Zustandsdiffusion verwendet, die sich stark an den Größen für die Einzellipiddiffusion anlehnen, jedoch durchweg ein wenig unter diesen liegen: $D_{\text {gel }} \approx 3 \cdot 10^{-11} \frac{\mathrm{cm}^{2}}{\mathrm{~s}}, D_{\text {fluid }} \approx 7 \cdot 10^{-9} \frac{\mathrm{cm}^{2}}{\mathrm{~s}}$ [127] [90]. Zur Berechnung des Diffusionskoeffizienten in Umwandlungsbereich wird wieder die "fluid fraction" zur Hilfe genommen:

$$
D=D_{\text {gel }} \cdot(1-f)+D_{\text {fluid }} \cdot f \quad .
$$

Auf diese Weise kann unter Verwendung der charakteristischen Bhattacharjee-Ferrell-Frequenz $\omega_{B F}$ und den Diffusionskoeffizienten die Korrelationslänge berechnet werden.

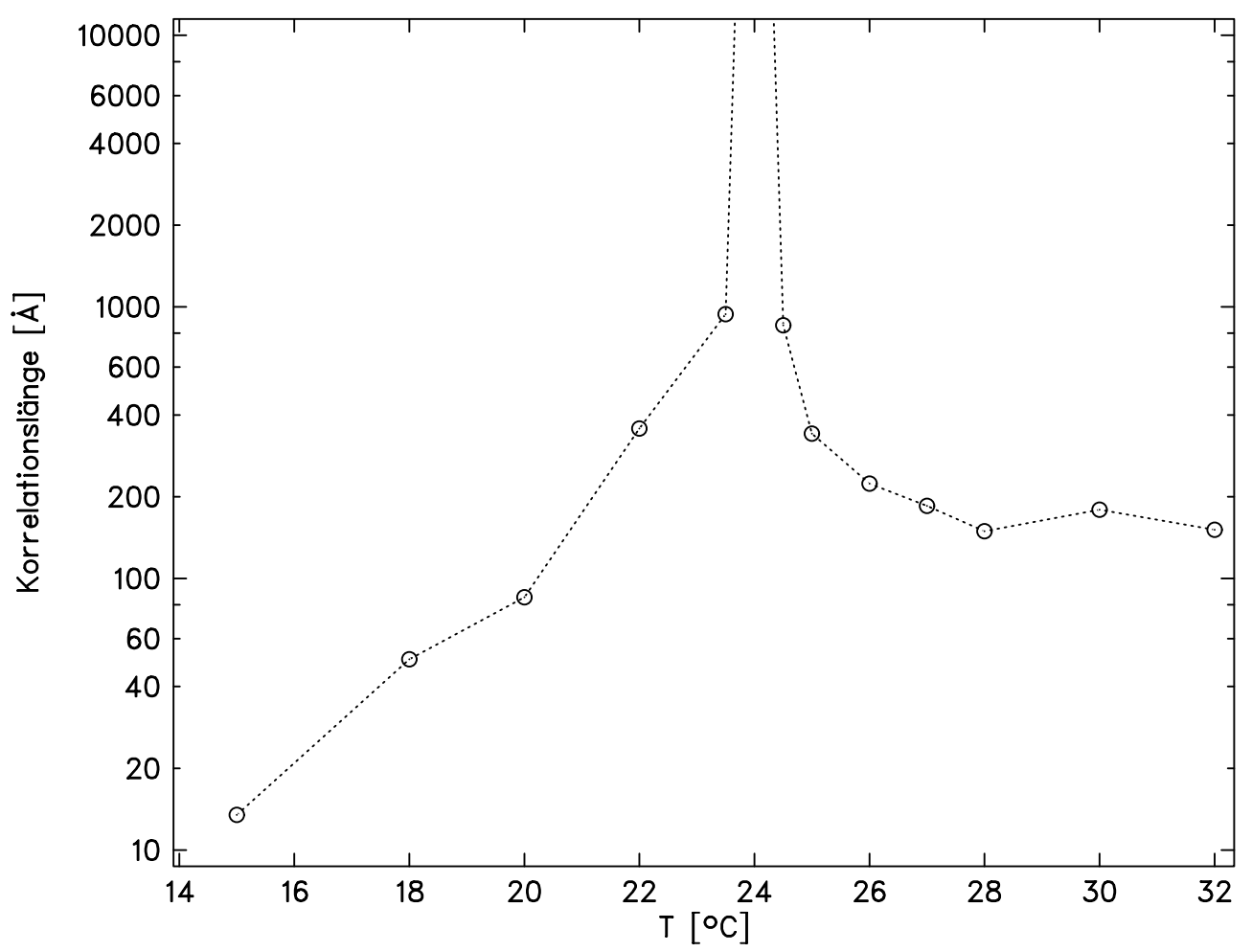

Abb. 7.22: Korrelationslänge der DMPC/Wasser Suspension

Abb. 7.22 zeigt den Verlauf der Korrelationslänge der DMPC/Wasser Suspension über der Temperatur. Deutlich ist ein starker Anstieg bei $T=T_{c}$ zu verzeichnen. Weiter ist zu registrieren, daß die Korrelationslänge unterhalb der Umwandlung 
deutlich niedriger liegt als oberhalb der Umwandlung. Dieses ist Ausdruck der hohen Fluidität nach dem Schmelzvorgang der Membran. Die Dynamik der Membran ist sehr viel schneller als im gelförmigen Zustand, und die Membran ist somit sehr viel schneller in der Lage, auf Veränderungen zu reagieren. Da bei $24^{\circ} \mathrm{C}$ die Bhattacharjee-Ferrell-Frequenz sehr klein ist, geht auch $\xi \Rightarrow \infty$. Konkret liegt die Korrelationslänge bei der Umwandlungstemperatur in der Größenordnung von $\xi \approx 2 \cdot 10^{6} \AA$. Dieser Werteverlauf liegt u.a. an dem als stetig angenommenen Verlauf des Diffusionskoeffizienten, der nach der Theorie von Bhattacharjee und Ferrell in der Umwandlung gegen Null gehen soll. Der mittlere Abstand zweier Lipide in der gelförmigen Phase beträgt etwa $d_{g e l}=3,8 \AA$ und in der fluiden Phase $d_{\text {fluid }}=4,5 \AA$ [53]. Somit liegt die Korrelationslänge bei $15^{\circ} \mathrm{C}$ bei etwa ein bis zwei Moleküllängen und steigt entsprechend bis zur Umwandlungstemperatur stark an. Weit oberhalb der Umwandlungstemperatur liegt diese Größe in etwa konstant bei 35 Moleküllängen. Dieser Werte relsutiert direkt aus dem Diffusionskoeffizienten $D_{\text {fluid }}$. Setzt man $D_{\text {fluid }}=D_{\text {gel }}$ so ergeben sich Korrelationslängen gleicher Größenordung im fluiden und gelförmigen Bereich. Genau bei $T=T_{c}$ ist die Korrelationslänge in jedem Fall derart angewachsen, daß ein Lipid am anderen Ende eines Vesikels die Bewegungen eines Lipides am gegenüberliegenden Punkt spürt. Der konkrete Zahlenwert steigt bei Auswertung der Spektren mittels der Theorie von Bhattacharjee und Ferrell weit über den Umfang der hier betrachteten Vesikel an, da die Theorie für reine dreidimensionale Systeme gemacht ist. In einem dreidimensionalen, kritischen System ist ein Wert von $\xi \approx 2 \cdot 10^{6} \AA$ eine realistische Größe. Hier jedoch muß statt dessen angenommen werden, daß die Moleküle eines Vesikels vollständig miteinander korreliert sind. Ausgehend von der Dimension eines Vesikels mit dem Durchmesser von ca. $100 \mathrm{~nm}$ müßte unter der Voraussetzung, daß die Korrelationslänge nicht länger als ein Vesikelumfang sein darf, der Diffusionskoeffizient bei $24^{\circ} \mathrm{C}$ bei ca. $D \approx 6 \cdot 10^{-15} \frac{\mathrm{cm}^{2}}{\mathrm{~s}}$ liegen. Dieses deutet ebenfalls auf eine sehr verlangsamte Dynamik des Systems am kritischen Punkt hin.

\subsubsection{Die Amplitude des Fluktuationsterms}

Eine quantitative Auswertung der Amplitude des Bhattacharjee-Ferrell-Terms nach den Beziehungen (5.31) - (5.33) scheint nicht sinnvoll, da es sich bei den Lipidsuspensionen nicht um vollständig dreidimensionale Flüssigkeiten im herkömmlichen Sinn handelt. Vielmehr bestehen die Suspensionen aus binären Mischungen von Lipidmembranen, die kritisches Verhalten zeigen, und Wasser als suspendierendes Medium, welches allerdings in dem hier betrachteten Temperaturbereich völlig unkritisch ist. Diese Tatsache ist in der Theorie von Bhattacharjee und Ferrell nicht berücksichtigt. Eine naheliegende Aufspaltung der Amplitude in einen Wasseranteil und einen kritischen Anteil, der durch die Lipide verursacht ist,

$$
A_{B F}=A_{W} \cdot\left(1-V_{b}\right)+A_{\text {Lipid }} \cdot V_{b},
$$

ist jedoch ebenfalls nur bedingt sinnvoll, denn die sicherlich vorhandene Kopplung der Wärme bzw. Wärmekapazität der Membran mit dem sie umgebenden Wasser ist nach wie vor nicht in den Gleichungen berücksichtigt. Die Kopplung der Wärme zwischen den beiden beteiligten Medien der binären Flüssigkeit ist jedoch evident, da die Wärmekapazität als eine grundlegende Größe der Bhattacharjee-Ferrell-Theorie 
angesehen werden muß. Auf der anderen Seite kann V. Invanona [67] zeigen, daß bei den hier verwendeten Frequenzen $(>200 \mathrm{kHz}$ ) kein merklicher Wärmeaustausch zwischen Wasser und Lipidmembran stattfindet.

Durch diese speziellen Eigenschaften der hier behandelten Lipidlösungen ist ebenfalls zu erklären, daß die charakteristische Frequenz der Fluktuationen $\omega_{B F}$ bei $T_{c}$ gegen Null geht, die Amplitude zwar groß wird jedoch nicht gegen unendlich geht (Abb. 7.20 und 7.21). Im Gegensatz dazu laufen die Geschwindigkeiten der kritischen Prozesse, die Fluktuationen in der Membran nach den Vorhersagen der Bhattacharjee-Ferrell-Theorie ab und folgen auch außerhalb der Umwandlung der Skalierungsfunktion (siehe Abb. 7.18).

\subsubsection{DMPC/DPPC Mischungen}

Das Verhalten der Parameter des an die Spektren der DMPC/DPPC Suspensionen angepaßten Bhattacharjee-Ferrell-Prozesses soll im folgenden diskutiert werden. Abb. 7.23 zeigt die normierte Amplitude des Bhattacharjee-Ferrell-Prozesses. Zum Vergleich ist zusätzlich zu den DMPC/DPPC Mischungen der Verlauf der DMPC/Wasser Suspension mit eingezeichnet. Auf den ersten Blick fällt die mit steigendem DPPC-Gehalt jeweils höhere Umwandlungstemperatur der jeweiligen Mischung auf. Wegen der guten Mischbarkeit von DMPC und DPPC kann dieses System als ideale binäre Mischung aufgefaßt werden. So ist es nicht verwunderlich, daß sich unter der Voraussetzung der jeweiligen Schmelzpunkte (DMPC bei $24,3^{\circ} \mathrm{C}$, DPPC bei $42^{\circ} \mathrm{C}$ ), die Umwandlungstemperatur proportional zum Mischungsverhältnis verschiebt. Die in etwa gleiche Höhe der Amplituden der verschiedenen Mischungen ist ebenfalls bemerkenswert. Dies deutet auf eine insgesamte Konsistenz mittels des gesamten Anpaßverfahrens mit einem Bhattacharjee-Ferrell-Prozeß und zwei Debye-Termen hin und ist somit als sinnvoll anzusehen. Bei genauerer Betrachtung fällt im weiteren auf, daß die Umwandlung, je ausgeglichener das Verhältnis von DMPC zu DPPC ist, um so breiter wird. Dieses ist völlig analog zu den Ergebnissen, wie sie in der Schallgeschwindigkeitsmessung gewonnen werden (siehe auch Abb. 3.6).

Abb. 7.24 zeigt die charakteristische Relaxationsrate $\omega_{B F}$ der DMPC/DPPC Suspensionen. Analog zur oben diskutierten Amplitude sinkt hier die Frequenz in der Umwandlung zu sehr kleinen Werten. Auch hier ist die Erhöhung der Umwandlungstemperatur mit steigendem DPPC Gehalt zu sehen, und eine Verbreiterung der Umwandlung bei der ausgeglichenen Mischung ist zu verzeichnen.

Die charakteristische Relaxationsrate $\omega_{B F}$ der DMPC/DPPC Mischungen ist über weite Temperaturbereiche sehr klein und somit folgt eine hohe Korrelationslänge über dem gleichen Temperaturbereich. Besonders ausgeprägt ist dieses bei der DMPC/DPPC 50:50 Suspension, bei der $\omega_{B F}$ über vier Temperaturen sehr klein ist.

\subsubsection{DMPC/Cholesterin Mischungen}

Im Gegensatz zu den DMPC/DPPC Mischungen ist hier keine starke Veränderung der Umwandlungstemperatur zu erwarten, sondern die charakteristischen Eigenschaften der Umwandlung sollten mit steigendem Cholesteringehalt verschwinden (siehe auch Abb. 2.7). 


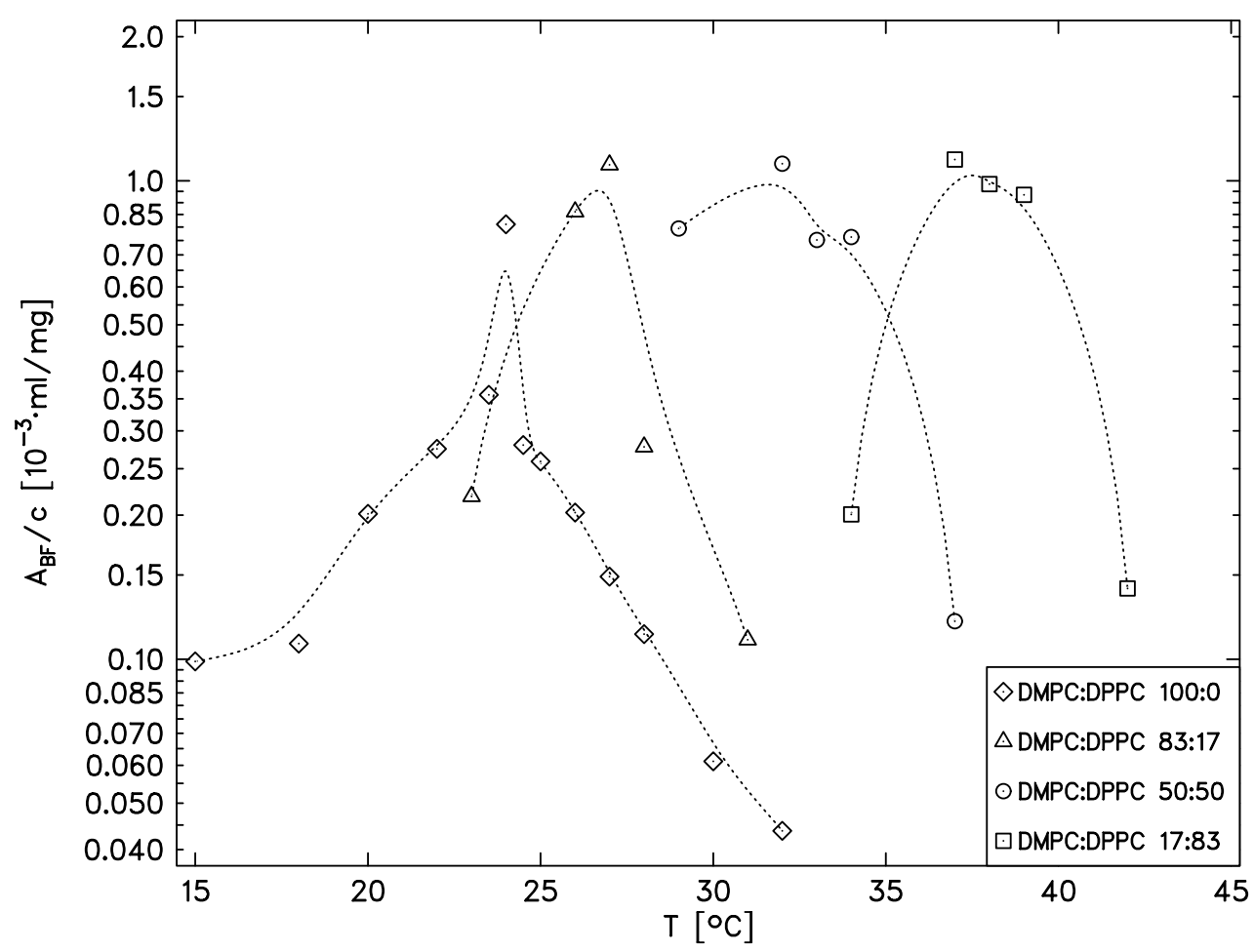

Abb. 7.23: Normierte Amplitude der BF-Spektralfunktion von reinem DMPC und den verschiedenen DMPC/DPPC Mischungen

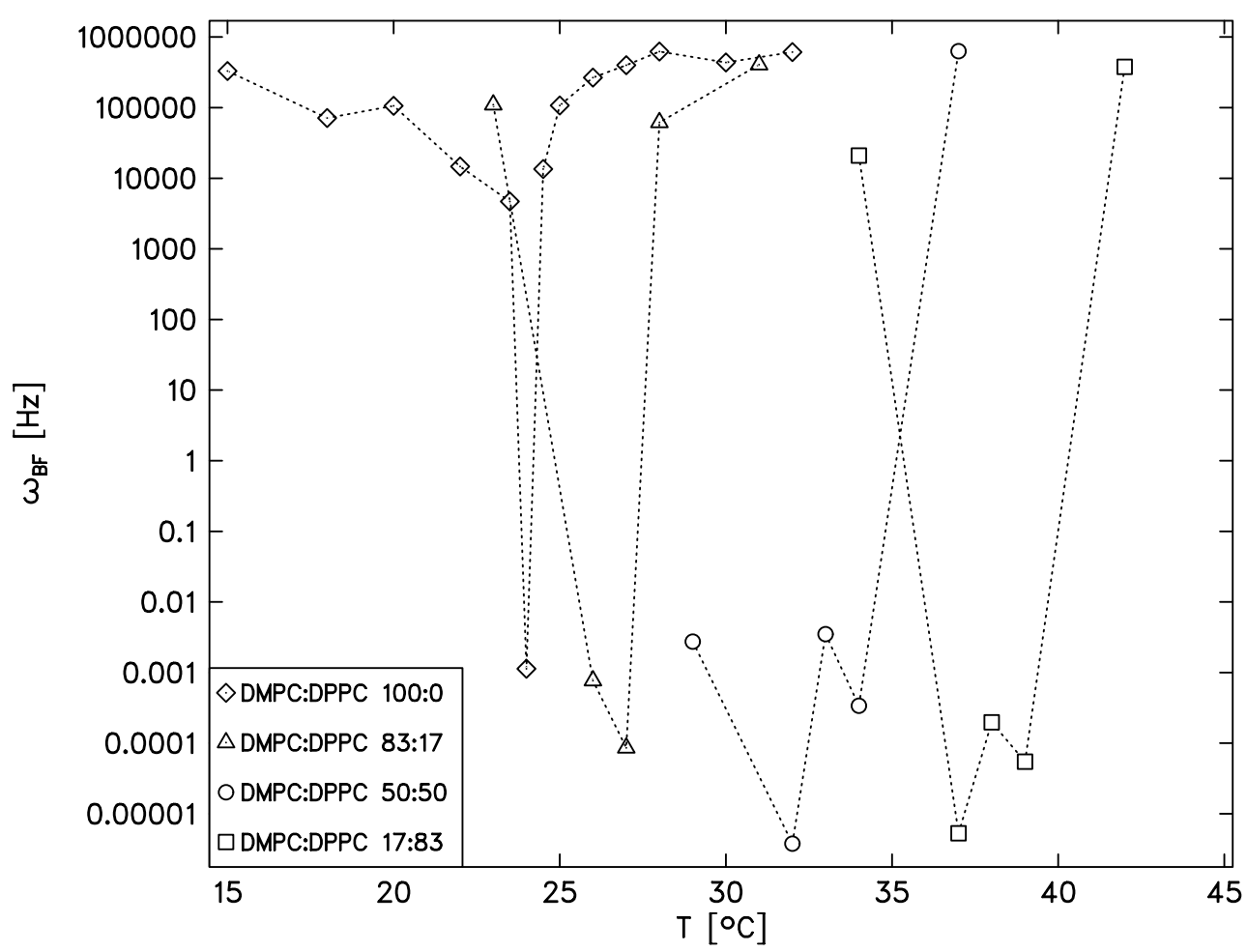

Abb. 7.24: $\omega_{B F}$ der BF-Spektralfunktion von reinem DMPC und den verschiedenen DMPC/DPPC Mischungen 


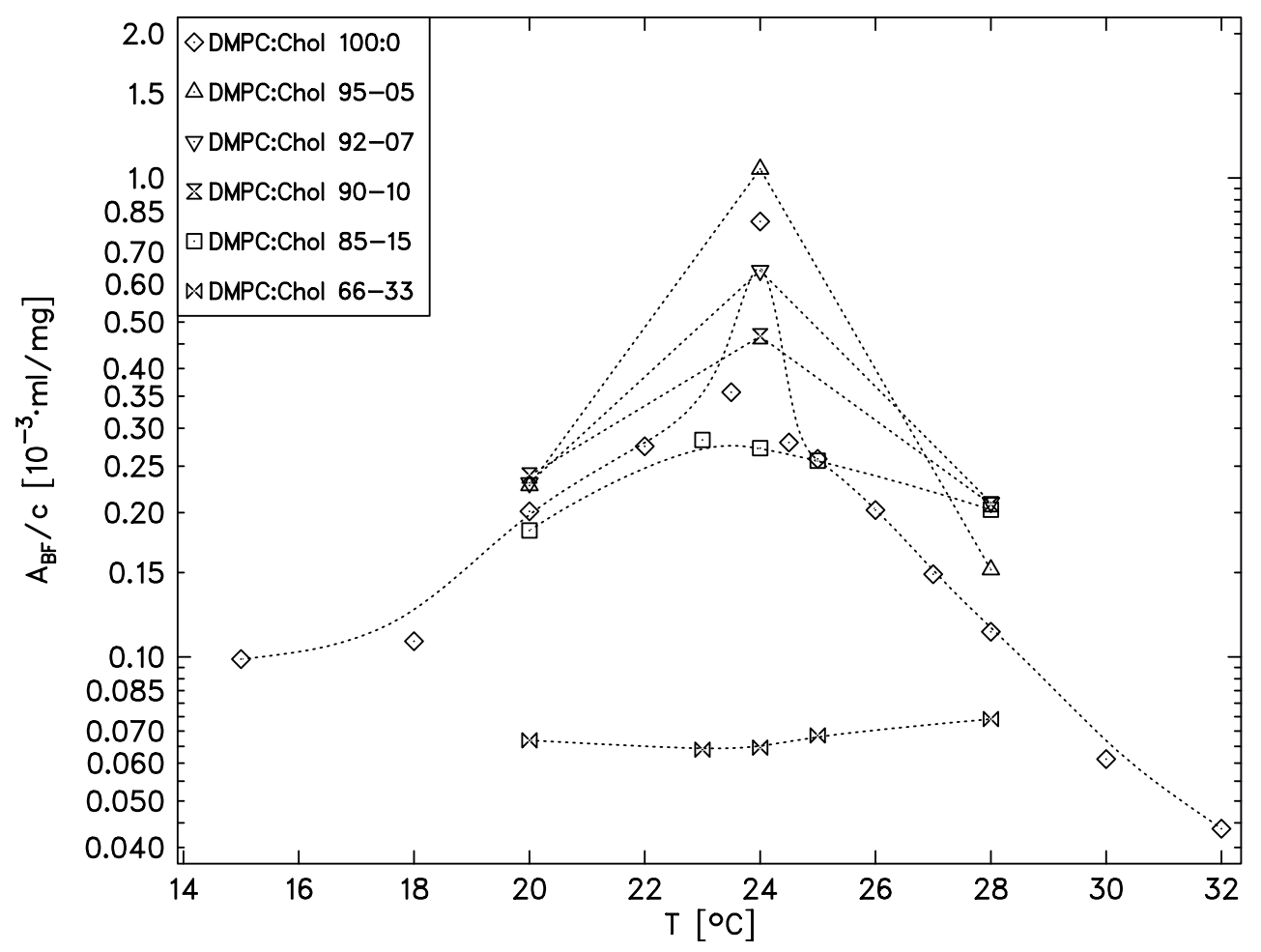

Abb. 7.25: Normierte Amplitude der BF-Spektralfunktion von reinem DMPC und den verschiedenen DMPC/Cholesterin Mischungen

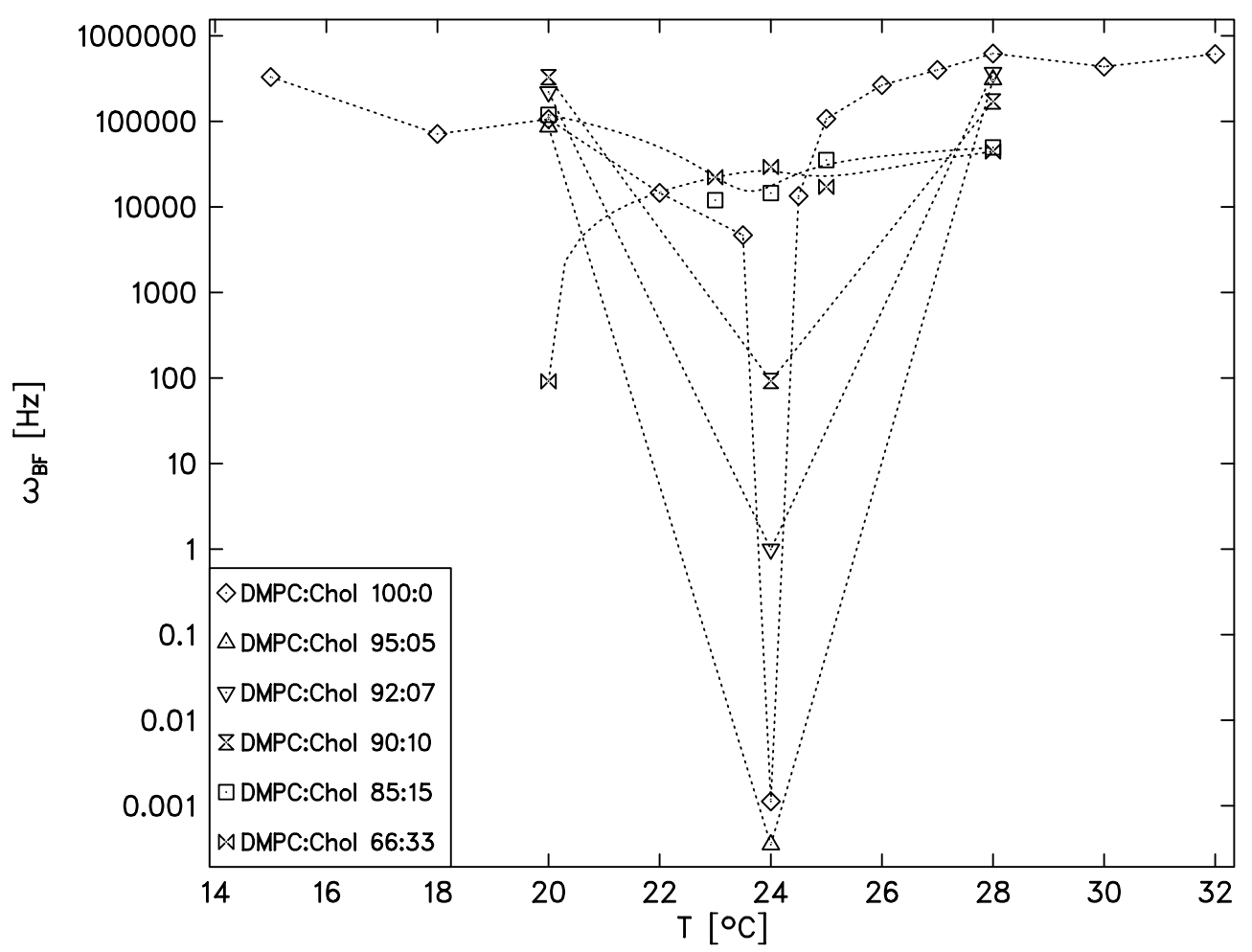

Abb. 7.26: $\omega_{B F}$ der BF-Spektralfunktion von reinem DMPC und den verschiedenen DMPC/Cholesterin Mischungen 
DMPC/Cholesterin 95:05 Relativ zur DMPC/Wasser Suspension scheint auf den ersten Blick keine Veränderung im Spektrum eingetreten zu sein. Die Amplitude zeigt ein Maximum bei $T_{c}$ und die Bhattacharjee-Ferrell-Frequenz wird ähnlich gering wie bei reinem DMPC. Dennoch ist sofort auffällig, daß sowohl die Amplitude größer als auch die Frequenz geringer als bei DMPC ist. Mouritsen et al. führt Monte-Carlo-Rechnungen an DPPL/Cholesterin Systemen durch [112]. Demnach ist Cholesterin bei geringer relativer Konzentration (bis ca. $8 \%$ ) weder in der Lage, die kristall-ähnliche Ordnung in der gelförmigen Phase zu brechen, noch einen höheren Ordnungsgrad in der fluiden Phase zu induzieren. Es trägt entgegen der Erwartungen zu einer verstärkten Bildung der Domänen bei, indem es sich an den Grenzen der Domänen anlagert. Cholesterin stabilisiert die Domänen und erhöht somit deren Langlebigkeit. Es muß jedoch auch erwähnt werden, daß die Fehler der Parameter des BhattacharjeeFerrell-Terms bei $T=T_{c}$ naturgemäß sehr hoch sind.

DMPC/Cholesterin 92,5:7,5 Hier ist sowohl die Amplitude als auch die Frequenz in etwa genauso groß wie bei reinem DMPC. Es scheinen sich ähnliche Prozesse wie bei der 95:05 Suspension abzuspielen. Jedoch ist die Amplitude gegenüber der 95:05 Suspension leicht gesunken.

DMPC/Cholesterin 90:10 Die Amplitude ist jetzt relativ zu reinem DMPC gesunken und die Bhattacharjee-Ferrell-Frequenz ist leicht erhöht. Es existiert nun zusätzlich zu den Domänen aus fluidem und gelförmigem Lipid eine cholesterinreiche Phase, die anscheinend an den Fluktuationen gar nicht oder nur in geringerem Maße teilnimmt. Das Cholesterin stabilisiert die Lipide und unterbindet so die charakteristische Umwandlung in diesen Bereichen der Membran. Zusätzlich behindert die Existenz dieser "liquid-ordered phase" die Fluktuationen der cholesterinarmen Bereiche.

DMPC/Cholesterin 85:15 Der Einfluß der cholesterinreichen Phase ist weiter gestiegen. Somit ist die Amplitude weiter gesunken und die BhattacharjeeFerrell-Frequenz weiter gestiegen. Immer mehr werden die Fluktuationen behindert und unterbunden. Die Charakteristik der Umwandlung, wie sie ganz deutlich bei der DMPC/Wasser Suspension zu sehen ist, verschwindet zusehends.

DMPC/Cholesterin 66:33 Im DMPC/Cholesterin Phasendiagramm in Abb. 2.7 wird der zweite eutektische Punkt überschritten. Demnach besteht die Membran vollständig aus der cholesterinreichen Phase, die keine oder fast keine Fluktuationen aufweist. Der Verlauf der Parameter zeigt deutlich, daß die Umwandlung und damit die Fluktuationen vollständig unterbunden sind. Beispielsweise ist die Amplitude des BF-Terms über der Temperatur annähernd konstant und auch die Bhattacharjee-Ferrell-Frequenz zeigt kein typisches Absinken bei $T=T_{c}$.

Aufgrund des Ansteigens von $\omega_{B F}$ mit steigendem Cholesteringehalt bei $T=T_{c}$ sinkt die Korrelationslänge. Für die Umwandlungstemperatur sind in der folgenden Tabelle die Korrelationslängen bei $T=T_{c}$ der DMPC/Cholesterin Suspensionen mit $D \approx 1 \cdot 10^{-9} \mathrm{~cm}^{2} / \mathrm{s}$ aufgeführt. 


\begin{tabular}{|c|c|c|c|c|c|}
\hline Suspension & $95: 05$ & $92,5: 7,5$ & $90: 10$ & $85: 15$ & $66: 33$ \\
\hline$\xi[\AA]$ & $258000(230000)$ & $4478(431)$ & $448(28)$ & $37(7)$ & $26(5)$ \\
\hline
\end{tabular}

Tabelle 7.8: Korrelationslängen der DMPC/Cholesterin Suspensionen bei $T=T_{c}$

\subsubsection{Mögliche Diskrepanzen der Bhattacharjee-Ferrell-Theorie}

In Kapitel 7.2.2 kann eindrucksvoll die Gültigkeit der Bhattacharjee-Ferrell-Theorie für das zeitliche Verhalten der Fluktuationen um $T_{c}$ gezeigt werden. Zudem muß die gute Anwendbarkeit der Theorie auf die Ultraschallspektren als zusätzliches Indiz für die Gültigkeit der Theorie gewertet werden. Dennoch darf nicht außer Acht gelassen werden, daß dieses nur für die hier betrachteten Frequenzen oberhalb von ca. $200 \mathrm{kHz}$ gilt. Durch die spezielle Form der Vesikel, die für sich betrachtet ein endliches, abgeschlossenes System bilden, ist durchaus eine mögliche Abweichung der Ultraschallabsorption von der Bhattacharjee-Ferrell-Theorie bei tieferen Frequenzen denkbar. Bhattacharjee bemüht sich um eine Modifikation der Theorie mit dem Ziel einer Anpassung an die Lipidsysteme [8]. Bei $T \approx T_{c}$ gilt demnach mit $\omega>>2 \Gamma \kappa^{z}$, $\kappa=\xi^{-1}, \Gamma=\omega_{B F}$ :

$$
(\alpha \lambda) \sim\left(\frac{1}{\omega}\right)^{\frac{\alpha}{z \nu}} .
$$

Dieses konnte in Kapitel 7.2.4 für DMPC gezeigt werden. Gilt $\omega<<2 \Gamma \kappa^{z}$, so gilt:

$$
(\alpha \lambda) \sim \frac{\omega}{\Gamma \kappa^{z}}
$$

Da offensichtlich bei den hier gemessenen Frequenzen nur die erste Bedingung erfüllt werden kann, bleibt die Frage, ob die Theorie auch für tiefere Frequenzen anwendbar ist, weiter unbeantwortet. Die gute Anwendbarkeit für den hier betrachteten Frequenzbereich ist jedoch festzuhalten.

\subsection{Mögliche molekulare Ursachen der Debye-Terme}

Keines der hier vorgestellten Ultraschallspektren kann ausschließlich mittels eines einzelnen Bhattacharjee-Ferrell-Term beschrieben werden. Bei einigen wenigen wird, wie z.B. bei DMPC/Cholesterin 85:15 und 66:33 ein und bei allen anderen werden zwei Debye-Relaxationsterme zusätzlich benötigt. Es zeigt sich, daß die Debye-Terme sehr wichtig für die Beschreibung der Spektren sind. Nur so kann eine in sich konsistente Anpassung bei allen Temperaturen, Mischungsverhältnissen und Konzentrationen erreicht werden. Zudem tragen die Einzelzeitrelaxationen mit einem nicht unerheblichen Beitrag zur gesamten Ultraschalldämpfung bei. Weiter zeigen Anpaßversuche mittels verschiedener Skalierungsfunktionen, daß sich weder die Amplitude noch die Relaxationszeit bei Verwendung einer anderen Skalierungsfunktion ändert (siehe Kapitel 7.2.1).

Es gilt nun, eine mögliche molekulare Ursache für die auftretenden Debye-Prozesse zu finden. Die für die Debye-Terme relevanten Relaxationszeiten liegen im Bereich von $10^{-9} \mathrm{~s}$ bis $10^{-6} \mathrm{~s}$ bzw. $10^{-10} \mathrm{~s}$ bis $10^{-8} \mathrm{~s}$. Aufgrund dieser Einschränkung kann eine 
Vielzahl von möglichen Relaxationsursachen ausgeschlossen werden. Die transversale Diffusion, also der "flip-flop" eines Lipides von der einen Membranseite zur anderen Seite kann als mögliche Ursache verworfen werden, da die Zeiten bei diesem Effekt in der Größenordung von Stunden oder Tagen liegen [126]. Die gesamte Membran ist in der Lage, Wellenbewegungen auszuführen. Da die dabei relevanten Zeiten bei einigen Sekunden liegen, kann auch dieser Effekt ausgeschlossen werden [128]. Außerdem kommt ein Austausch von Monomeren als mögliche Ursache nicht in Betracht, da die kritische Mizellkonzentration (ca. $10^{-10} \mathrm{~mol} / \mathrm{l}$ ) bei DMPC-Suspensionen extrem niedrig ist. Die zu erwartenden Zeiten korrespondieren mit Frequenzen weit unterhalb des hier gemessenen Frequenzbereiches [96].

Eine früher oft diskutierte mögliche Ursache für die Debye-Terme ist die Kopfgruppenumlagerung der Lipide [48]. Der charakteristische Verlauf der Relaxationszeit dieses Prozesses über der Temperatur ist jedoch in Kap. 4.4 mittels dielektrischer Spektroskopie sehr genau ermittelt worden (siehe auch Abb. 4.16). Keiner der in der Ultraschallspektroskopie auftretenden Debye-Terme zeigt einen vergleichbaren Temperaturgang. Somit kann die Kopfgruppenumlagerung als molekulare Ursache ausgeschlossen werden. Dennoch besteht die Möglichkeit, daß das entsprechend zur Kopfgruppe vorhandene Hydratwasser im Ultraschallspektrum als Relaxationsprozeß sichtbar ist. Denkbar ist nach erfolgter Umorientierung der Kopfgruppe das Nachrelaxieren der die Kopfgruppe umgebenden Wassermoleküle, da das für die Hydratisierung benötigte Wasser sicherlich andere Eigenschaften im Bezug auf die Viskositäten $\eta_{s}, \eta_{v}$ besitzt.

Um zu einer möglichst realistischen Erklärung der Debye-Terme zu kommen, werden die Meßergebnisse für verschiedene Membranzusammensetzungen betrachtet. Aus den charakteristischen Verläufen von Amplitude und Relaxationszeit soll im folgenden auf die molekulare Ursache der Debye-Terme geschlossen werden.

\subsection{Der tieffrequente Debye-Term}

Die an die Spektren angepaßte Bhattacharjee-Ferrell-Funktion beschreibt die in der Umwandlung auftretenden Fluktuationen. Es koexistieren gelförmige und fluide Lipide innerhalb von Domänen nebeneinander auf einem Vesikel. Jedoch hat man sich die Diffusion der Domänen nicht als wirkliche Bewegung eines kompletten Areals auf der Membran vorzustellen. Vielmehr scheint es so zu sein, daß man von einer Diffusion von Zuständen sprechen muß (siehe auch Kapitel 7.2.6).

Unter diesem Aspekt scheint es möglich, daß die laterale Diffusion von einzelnen Molekülen innerhalb der Matrix im Ultraschallspektrum gesondert sichtbar ist, sofern dieser Prozeß genügen Reaktionsvolumen aufweist. Aufgrund der selbst in der fluiden Phase noch relativ hohen Ordnung innerhalb der Membran muß für eine laterale Bewegung eines Lipides erst ein genügend großes Volumen frei werden. Somit ist die laterale Diffusion abhängig von der Dichte in bzw. dem Druck auf die Membran und könnte somit zu einer Absorption der Ultraschallwelle beitragen. Die Werte für die Sprungfrequenzen liegen nach Marsh [99] im Bereich von einigen $f_{s p} \approx 10^{7} \mathrm{~Hz}$. Somit scheint dieser Effekt durchaus als mögliche Ursache für den tieffrequenten Debye-Prozeß in Betracht zu kommen. 


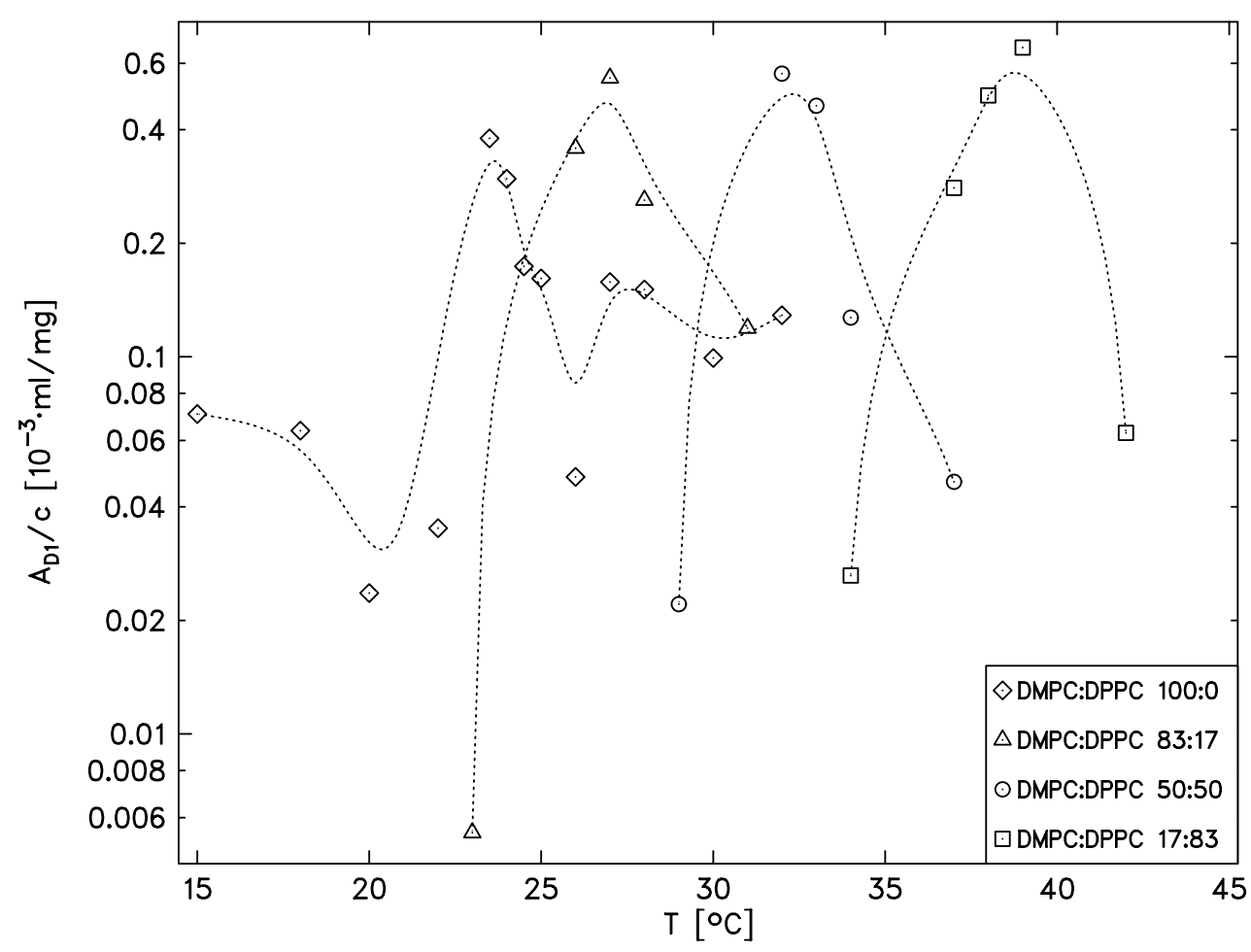

Abb. 7.27: Normierte Amplitude des tieffrequenten Debye-Terms von reinem DMPC und den verschiedenen DMPC/DPPC Mischungen

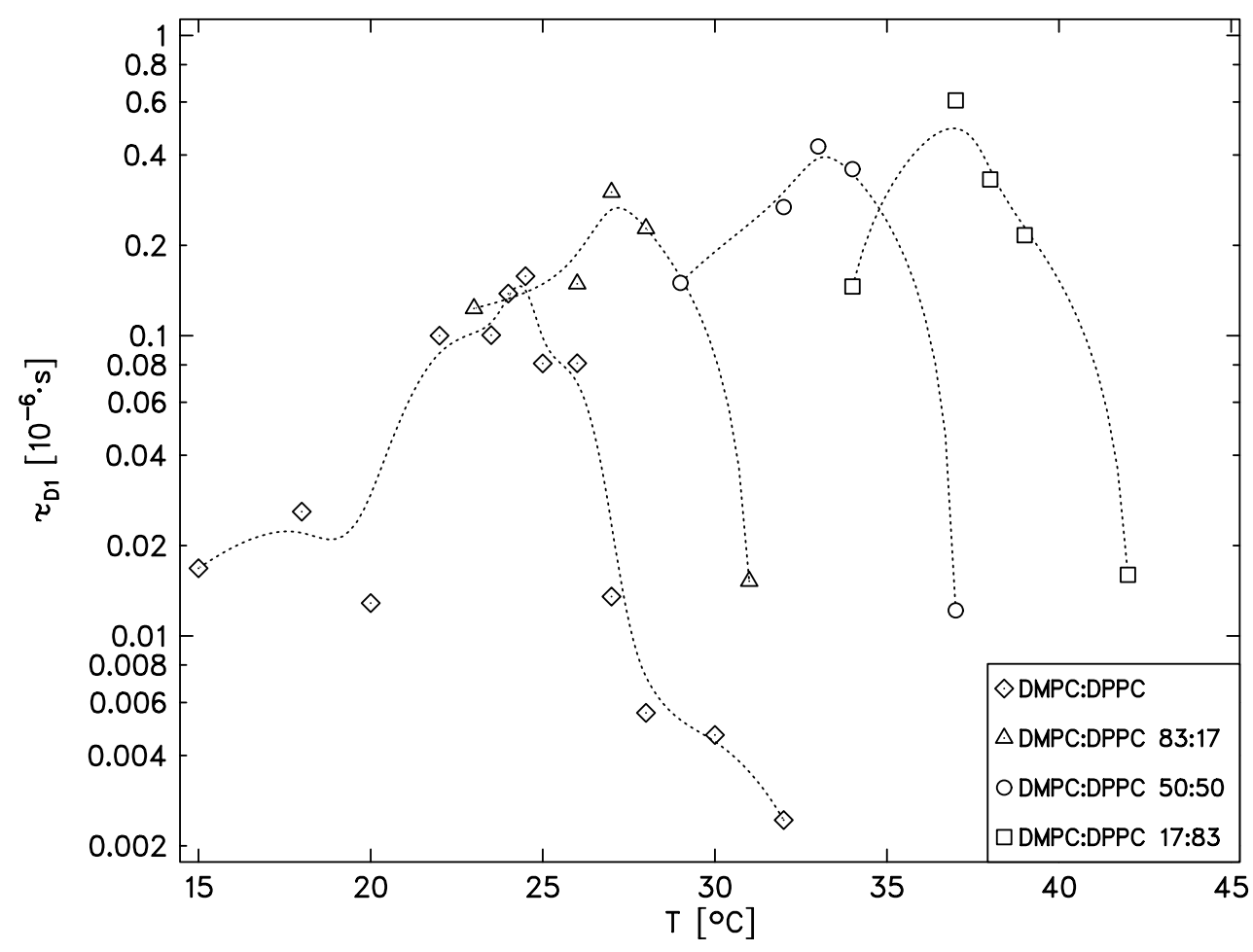

Abb. 7.28: Relaxationszeit des tieffrequenten Debye-Terms von reinem DMPC und den verschiedenen DMPC/DPPC Mischungen 


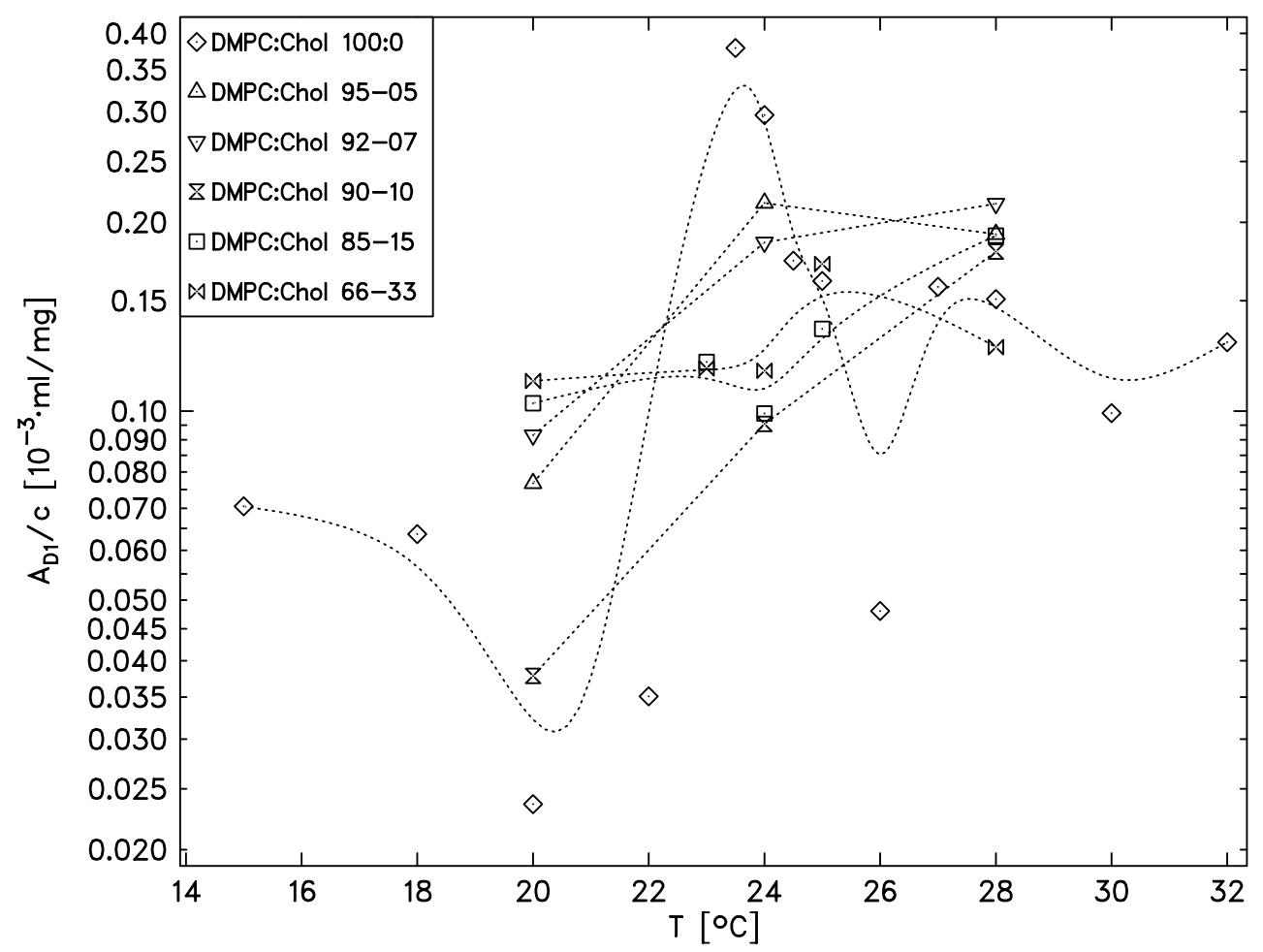

Abb. 7.29: Normierte Amplitude des tieffrequenten Debye-Terms von reinem DMPC und den verschiedenen DMPC/Cholesterin Mischungen

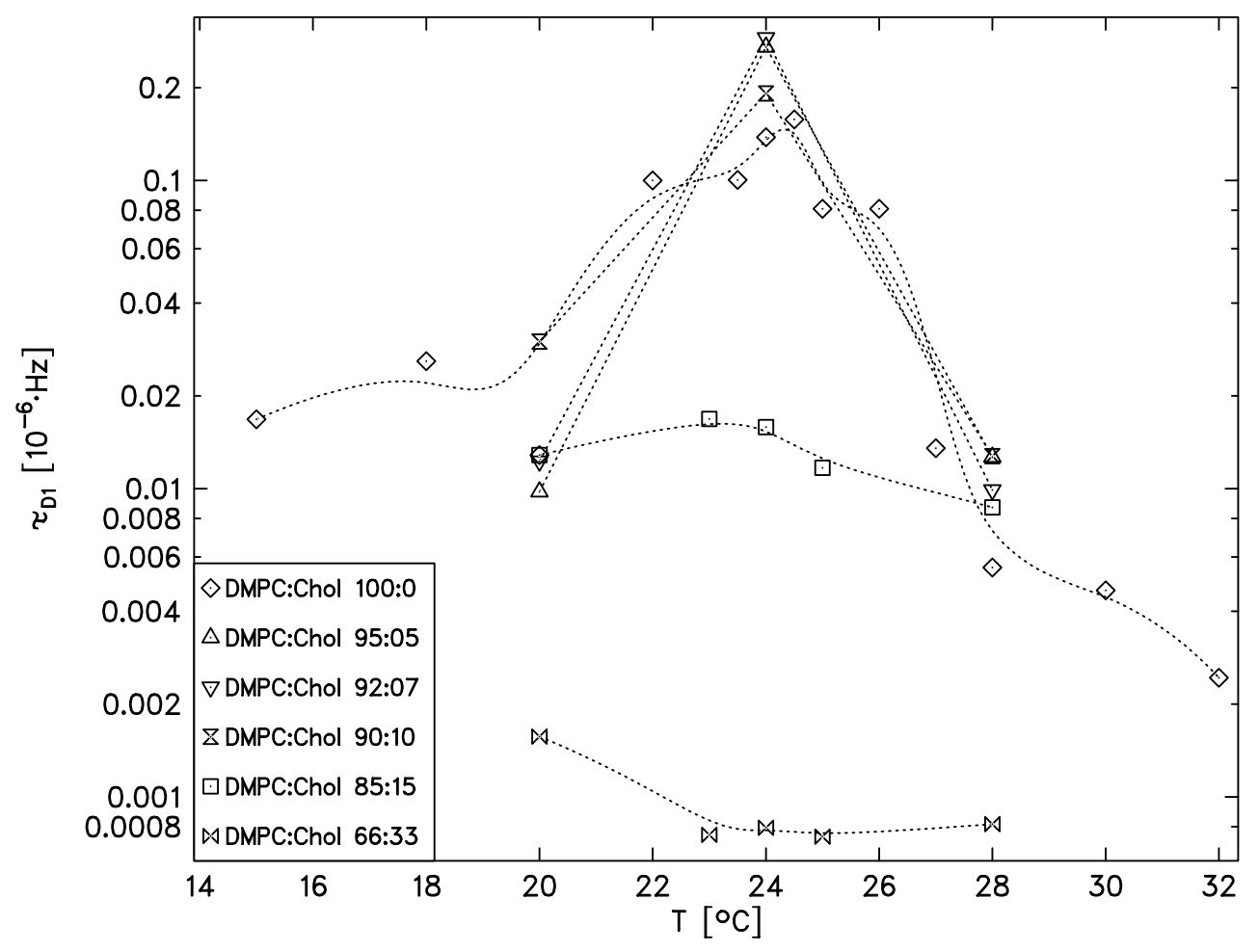

Abb. 7.30: Relaxationszeit des tieffrequenten Debye-Terms von reinem DMPC und den verschiedenen DMPC/Cholesterin Mischungen 


\subsubsection{Die Viskosität nach Eyring}

Nach Eyring [27] sind in einer Flüssigkeit die einzelnen Moleküle im Rahmen der thermischen Bewegungen relativ dicht gepackt. Durch plötzliche thermische Stöße, kann diese Ordnung für die Entstehung eines "Loches", eines "freien Gitterplatzes", aufgebrochen werden. Dieser frei gewordene Raum kann nun von ein angrenzendes Molekül eingenommen werden. Anschließend schließt sich das übriggebliebene Loch durch die thermische Dynamik der Flüssigkeit. Dabei ist es nicht zwingend, daß in ein entstandenes Loch auch ein neues Molekül hineingerät. Das gleiche Molekül kann durchaus erneut seinen alten Platz einnehmen.

Letztendlich ist es möglich, über die maxwellsche Wahrscheinlichkeitsverteilung und mittels Annahme einer virtuell angelegten Scherspannung einen Zusammenhang zwischen Viskosität und Sprungfrequenz der Moleküle herzustellen:

$$
\eta=\frac{k T}{\alpha_{V}} \cdot\left(\nu^{-1}\right), \quad \alpha_{V}=L_{y} \cdot L_{3}^{2}
$$

Dabei ist $L_{3}$ der Abstand der Moleküle und $L_{y}$ ein Maß für die angelegte Scherspannung. Überlicherweise haben $L_{y}$ und $L_{3}$ nicht genau die gleiche Größe. Nur bei kugelförmigen oder kubischen Molekülgeometrien kann $L_{y}=L_{3}$ gesetzt werden.

Die Verbindung zu dem Meßergebnissen der Ultraschallspektroskopie besteht darin, daß die Sprungfrequenz $\nu$ mit der Relaxationszeit bzw. der Relaxationsfrequenz des tieffrequenten Debye-Terms in Übereinstimmung zu bringen ist.

Führt man an Stelle der angelegten Scherspannung, die Anzahl der vorhandenen Löcher pro Mol $N_{H}$ und deren Volumen $v_{H}$ ein, so ist noch eine Modifikation von Gleichung (7.16) möglich. Mit der Wahrscheinlichkeit $N_{H} v_{H} / N v_{0}$ für einen Sprung in ein vorhandenes Loch, wobei $\mathrm{N}$ die Anzahl der Moleküle und $v_{0}$ das Volumen eines Moleküls ist, ergibt sich folgende Beziehung:

$$
\eta=\frac{R T}{N_{H} v_{H}} \cdot \tau
$$

\subsubsection{Diffusionskoeffizienten}

Die bekannten Diffusionskoeffizienten von einzelnen Lipiden in Membranen liegen alle im Bereich von $D_{\text {gel }} \approx 1 \cdot 10^{-10} \mathrm{~cm}^{2} / \mathrm{s}$ in der gelförmigen Phase bis $D_{\text {fluid }} \approx$ $1 \cdot 10^{-8} \mathrm{~cm}^{2} / \mathrm{s}$ in der fluiden Phase [132] [90] [95] [116] [19] [143] [144] [145] [146] [1]. Mittels der Einstein-Stokes-Beziehung kann aus den errechneten Viskositäten ein Diffusionskoeffizient ermittelt werden. Die Viskositäten sind in Tabelle $7.11 \mathrm{zu}$ finden.

$$
D=\frac{k T}{6 \pi \eta r}
$$

Durch Verwendung des mittleren lateralen Netzebenenabstandes der Lipide von $L_{3} \approx 4,2 \AA[127]$ und $L_{y} \approx L_{3}$, können mit Gleichung (7.16) und (7.18) unter Benutzung der Relaxationszeiten des tieffrequenten Debye-Terms Diffusionskoeffizienten ausgerechnet werden. Sie sind für die reine DMPC Suspension und die DMPC/Cholesterin Suspensionen in Abb. 7.31 dargestellt. Es fällt sofort ins Auge, daß die Größenordnung der hier errechneten Werte von reinem DMPC mit denen 


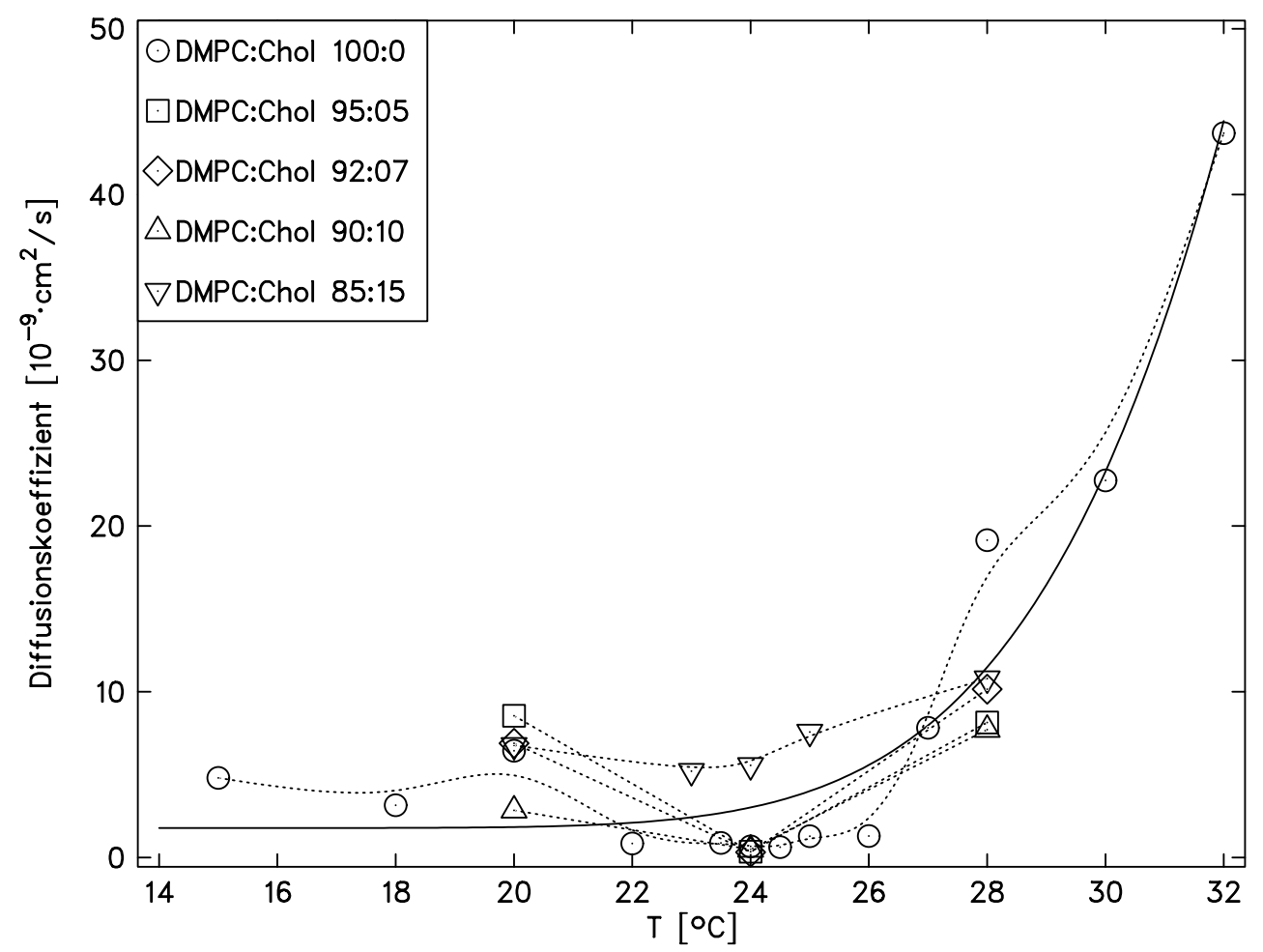

Abb. 7.31: Aus den Relaxationszeiten des tieffrequenten Debye-Terms errechneten Diffusionskoeffizienten der DMPC und DMPC/Cholesterin Suspensionen; die durchgezogene Linie zeigt den Verlauf der an die DMPC Werte angepaßten Funktion (7.20)

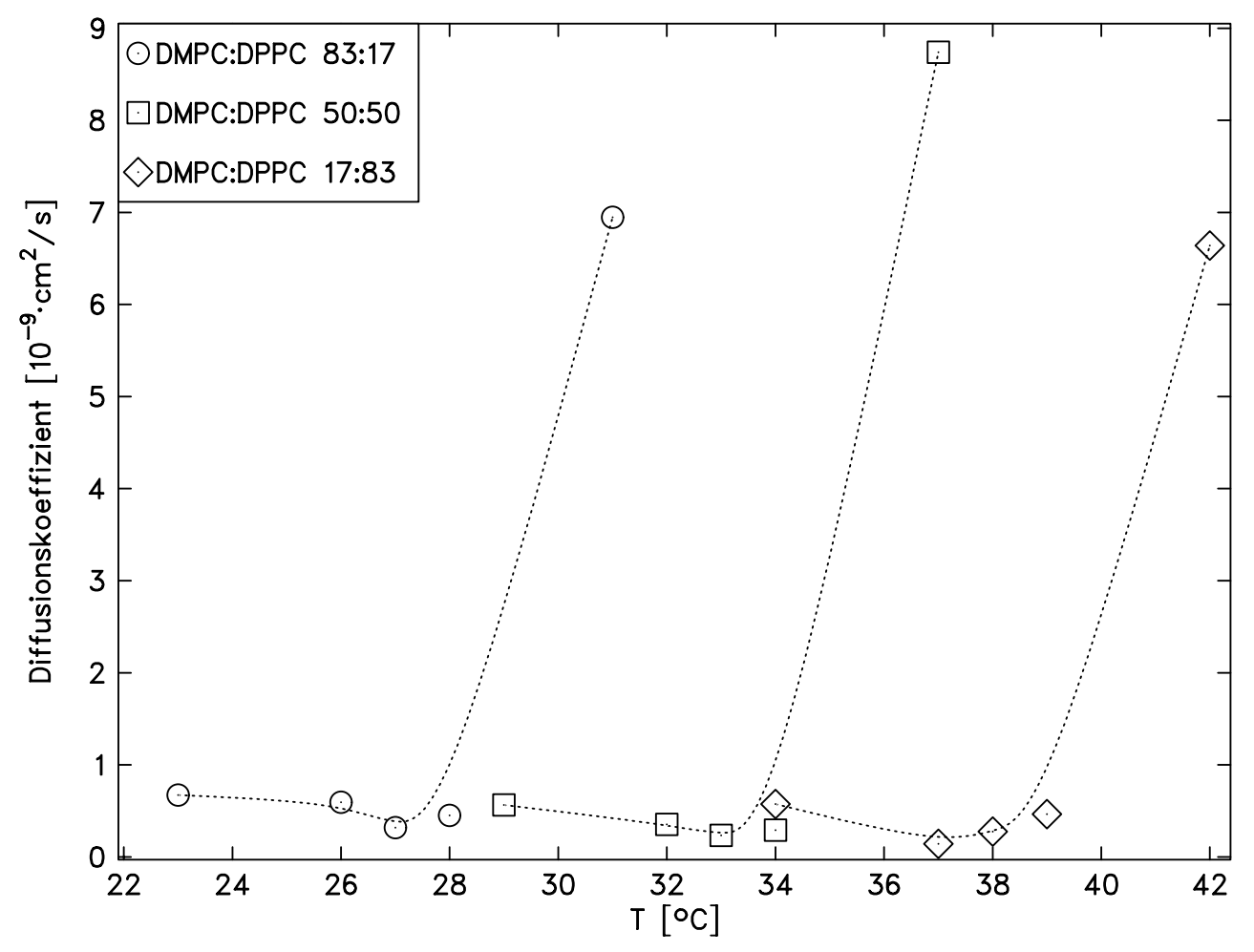

Abb. 7.32: Aus den Relaxationszeiten des tieffrequenten DebyeTerms errechneten Diffusionskoeffizienten der DMPC/DPPC Suspensionen 
von P. Schwille et al. [132] [90] durch FRAP-Experimente direkt ermittelten Werte übereinstimmen. Unterhalb der Umwandlungstemperatur liegen die Werte bei $D \approx 1 \cdot 10^{-10} \mathrm{~cm}^{2} / \mathrm{s}$ bis $1 \cdot 10^{-9} \mathrm{~cm}^{2} / \mathrm{s}$ während sie oberhalb der Umwandlung stark ansteigen und bei Werten um $D \approx 3 \cdot 10^{-8} \mathrm{~cm}^{2} / \mathrm{s}$ liegen. In der Umwandlung sinken die Diffusionskoeffizienten leicht ab, was durch das kritische Verhalten bedingt scheint. Die Existenz von Domänen verschiedenen Typs, also gelförmige und fluide Phasen, scheint die Diffusion der einzelnen Lipide zu behindern. Anscheinend ist es einem Molekül nicht so einfach möglich von der Phase eines Typs in die Phase des anderen Type zu diffundieren. Dieses resultiert schließlich in einem geringeren Diffusionskoeffizienten in der Umwandlung. Abb. 7.32 zeigt die errechneten Diffusionskoeffizienten der DMPC/DPPC Suspensionen. Auch hier ist ein starkes Ansteigen oberhalb der jeweiligen Umwandlungstemperatur zu beobachten. Die Berechnung der Diffusionskoeffizienten mittels der alternativen Gleichung (7.17) gelingt ebenfalls und führt für Werte von $N_{H} \approx 0.05$ bis 0.4 zum gleichen Ergebnis. Bei P. Schwille et al. [132] [90] werden auch Diffusionskoeffizienten von Membranen ermittelt, welche Cholesterin enthalten. Es zeigt sich dort, daß der Diffusionskoeffizient mit steigenden Cholesteringehalt von $D \approx 5 \cdot 10^{-8} \mathrm{~cm}^{2} / \mathrm{s}$ bis auf $D \approx 3 \cdot 10^{-9} \mathrm{~cm}^{2} / \mathrm{s}$ bei einem 50 prozentigem Cholesteringehalt absinkt. In Abb. 7.31 ist dieses ansatzweise zu sehen. Mit steigendem Cholesteringehalt verschwindet das Absinken des Diffusionskoeffizienten in der Umwandlung. Die DMPC/Cholesterin 85:15 Suspension zeigt nur noch einen fast konstanten Verlauf über der Temperatur. Leider ergeben die Berechnungen zur DMPC/Cholesterin 66:33 Suspension um eine Größenordnung zu hohe Werte für $D$. Die Ursache dafür liegt einerseits in der starken Streuung der Punkte der entsprechenden Ultraschallspektren und der damit verbundenen Unsicherheit bei den Anpaßrechnungen, andererseits ist die Verwendung der oben genannten Abstände möglicherweise unzulässig, da in solch einer Lipidmischung sicherlich Moleküle verschiedener Eigenschaften und Größen miteinander interagieren. Es kann bei diesen Mischungsverhältnissen nicht mehr von einer homogenen Phase gesprochen werden, die jedoch für die Berechnung mittels der obigen Beziehungen vorausgesetzt werden muß. Zudem ist bekannt, daß eine cholesterinreiche Phase existiert, in der Cholesterinmoleküle recht fest an die Lipide gebunden sind und eventuell ein CholesterinLipid-Paar als ein einziges Molekül betrachtet werden muß.

\section{Diffusionskoeffizienten bei $T_{m}$}

Abb. 7.31 zeigt eindeutig, daß der aus den Relaxationszeiten berechnete Diffusionskoeffizent bei $T_{m}$ gegenüber der reinen Gel-Phase bzw. fluiden Phase leicht abgesenkt ist. Dieses ist zunächst eine direkte Folge aus dem Temperaturverlauf der Relaxationszeiten (siehe Abb. 7.28), die ihrerseits bei $T_{m}$ ein Maximum aufweisen. Möglicherweise hat der besondere Zustand der Membran in der Nähe von $T_{m}$ Einfluß auf die Diffusionsvorgänge der einzelnen Lipide. In der Umwandlung bilden sich Domänen aus fluiden und gelförmigen Lipiden verschiedener Größen. Es existieren dabei sowohl sehr große Domänen als auch sehr kleine, während die Domänengröße weitab von $T_{m}$ zunehmend abnimmt. Somit erreicht neben der reinen Anzahl der Domänen auch die Anzahl der Schnittstellen zwischen den beiden Domänentypen bei der Umwandlungstemperatur ein Maximum. Es ist nun denkbar, daß die Diffusion der einzelnen Lipide über eine solche Phasengrenze im Vergleich zur Diffusion innerhalb einer relativ großen Domäne oder im reinen fluiden Bereich erheblich schwerer 
erfolgt. Ein Lipid, daß eine solche Phasengrenze überquert, gelangt in eine Domäne des anderen Typs, was für das Lipid energetisch sehr ungünstig ist. Ein gelförmiges Lipid, welches sich in einer fluiden Domäne wiederfindet, müßte sich nicht nur in eine völlig andere Umgebung in Bezug auf den Zustand der Alkylketten einfinden, es müßte auch eine erhebliche Hydrathülle mitziehen, da das gelförmige Lipid eine größere Länge aufweist als die fluiden Lipide. Alle diese Überlegungen machen eine solche Überquerung einer Phasengrenze extrem unwahrscheinlich. Es ist wahrscheinlicher, daß das Lipid bei der Diffusion in eine andere Domäne den Zustand wechselt.

\subsubsection{Die Amplitude}

In Abb. 7.27 und Abb. 7.30 sind die normierten Amplituden des tieffrequenten Debye-Terms dargestellt. Über Gleichung (5.10) ist die gemessene Amplitude u.a. mit dem Reaktionsvolumen verknüpft. Es ist seit langem bekannt, daß die Permeabilität der Membran für Wasser bei $T_{m}$ ein Maximum aufweist [127] [128] [126]. Möglicherweise ist das für den tieffrequenten Debye-Term gefundene Maximum bei $T_{m}$ ein Hinweis für das mögliche Vorhandensein einer solchen Permeabilität. Bei der Umwandungstemperatur existiert demzufolge ein Maximum ein Löchern gemäß Eyrings Diffusionsmodells [27]. Diese Löcher hätten zusätzlich eine maximale Lebensdauer, was letztendlich in einer maximalen Permeabilität resultieren könnte. Jedoch muß gesagt werden, daß diese Überlegungen an dieser Stelle rein spekulativ sind.

\subsubsection{Diffusion nach Cohen und Thunball}

Detaillierte Untersuchungen der lokalen Diffusion an Modellmembranen aus Lipiden verschiedener Kettenlänge zeigen [127]:

- Die Diffusionskoeffizienten in Modellmembranen aus Diacylphosphatidylcholinen sind im wesentlichen unabhängig von der Kettenlänge der beteiligten Lipide und allein eine Funktion der Temperatur.

- Im fluiden Zustand liegt der Diffusionskoeffizient bei etwa $D \approx 5 \cdot 10^{-8}$ bis $1 \cdot 10^{-7} \mathrm{~cm}^{2} / \mathrm{s}$ und im gelförmigen Zustand bei etwa $D \approx 2 \cdot 10^{-11} \mathrm{~cm}^{2} / \mathrm{s}$ bis $5 \cdot 10^{-10} \mathrm{~cm}^{2} / \mathrm{s}$ [127] [90].

- Die Temperaturabhängigkeit gehorcht im fluiden Zustand einem exponentiellem Gesetz.

$$
D=D_{0} \cdot \exp \left(-\frac{A}{T}\right)
$$

Cohen und Thunball entwickeln nun ein Modell [19], welches die Diffusion in einer Modellmembran beschreibt. Dabei wird ausgehend vom freien Löcher Modell (siehe Kap. (7.4.1)) ein Ausdruck hergeleitet, der die Temperaturabhängigkeit der Diffusion bei vielen Systemen gut beschreiben kann. Der elementare Prozeß ist die Schaffung freien Volumens $v_{f}$ in der Lipidschicht durch laterale Dichteschwankungen. Ein Lipid, welches an ein so entstehendes Loch grenzt, kann nun von seinem alten Platz in die Lücke springen, falls dessen Volumen eine kritische Größe $v_{k}$ überschreitet. Die 
Rückkehr in die alte Position wird durch das Nachrücken anderer Membranlipide verhindert. Die hier auftretende Sprungfrequenz wird im wesentlichen durch zwei Faktoren bestimmt:

- Durch die mittlere Zeit, die die Schaffung der Lücke mit dem Volumen $v_{f}$ in Anspruch nimmt.

- Durch die gaskinetische Geschwindigkeit $u$ des betrachteten Teilchens in seinem Lösungsmittelkäfig.

Die Übertragung des freien Volumenmodells auf zweidimensionale Systeme ergibt schließlich:

$$
D=g \cdot a \cdot u \cdot \exp \left(\frac{-\gamma a^{k}}{a_{f}}\right)
$$

mit

$$
a_{f}=\alpha a_{m}\left(T-T_{0}\right)
$$

Dabei ist $g$ ein geometrischer Faktor mit $g \approx 1, a$ der Durchmesser eines Gitterplatzes, $a_{k}$ die kritische freie Fläche, die für einen erfolgreichen Diffusionssprung nötig ist, $u$ die kinetische Gasgeschwindigkeit $(u=\sqrt{2 k T / m}), m$ die Masse des Moleküls und $\gamma$ ein Faktor zwischen 0,5 und 1. Er wird eingeführt, um dem Überlapp von freiem Volumina Rechnung zu tragen. Die Temperaturabhängigkeit der Diffusion ist hauptsächlich eine Folge der lateralen Ausdehnung der Membran. Die freie Molekülfläche ist gegeben durch $a_{f}=\alpha a_{m}\left(T-T_{0}\right)$, wobei $a_{m}$ die Fläche bei der Schmelztemperatur und $\alpha$ der laterale, thermische Ausdehnungskoeffizient ist. Mit $T_{0}$ ist hier nicht die Hauptumwandlungstemperatur sondern vielmehr die Temperatur, bei der kein freies Volumen für ein Lipid zur Verfügung steht, gemeint.

Gleichung (7.20) beschreibt den Temperaturgang der Diffusion im fluiden Zustand recht gut. Die exponentielle Natur von Gleichung (7.20) führt jedoch bei einem Absinken unterhalb von $T_{m}$ zu sehr kleinen Werten nahe Null. Um den gelförmigen Bereich nicht aussparen zu müssen, wird Gleichung (7.20) um einen konstanten Beitrag erweitert:

$$
D=D_{0}+g \cdot a_{k} \cdot u \cdot \exp \left(\frac{-\gamma a^{k}}{a_{f}}\right)
$$

Leider kann dieses Modell zunächst nicht direkt auf die hier gefundenen Meßergebnisse angewendet werden, da in dieser Form keine Identifikation mit einem Ultraschallrelaxationsprozeß über die Relaxationszeit möglich ist.

\section{Anwendung des freien Volumenmodells}

Um nun eine Beziehung zwischen den hier ermittelten Diffusionskoeffizienten und dem freien Volumenmodell von Cohen und Thunball herzustellen, wird im folgenden versucht, den Verlauf der Werte von DMPC mittels Gleichung (7.20) zu beschreiben. Mit dem lateralen Ausdehnungskoeffizienten $\alpha \approx 0.003$ [53], dem Durchmesser eines 


\begin{tabular}{|c|c|c|c|}
\hline$g$ & $\gamma a_{k} / a_{m}$ & $T_{0}\left[{ }^{\circ} \mathrm{C}\right]$ & $D_{0}\left[\mathrm{~cm}^{2} / \mathrm{s}\right]$ \\
\hline \hline $0,00061(79)$ & $0,047(54)$ & $22,6(38)$ & $2,2(13) \cdot 10^{-9}$ \\
0,3 & $0,59(10)$ & $6,8(45)$ & $1,8(17) \cdot 10^{-9}$ \\
1 & $0,77(14)$ & $3,6(52)$ & $1,8(17) \cdot 10^{-9}$ \\
0,3 & $0,63(8)$ & $4,8(33)$ & $5 \cdot 10^{-10}$ \\
\hline
\end{tabular}

Tabelle 7.9: Ergebnisse der Anpassungen an alle Punkte der DMPC/Wasser Suspension

Lipides von $a=4 \AA$ und der Molekülmasse von DMPC $(677.9 \mathrm{~g} / \mathrm{mol})$, kann die Funktion (7.20) an die ermittelten Werte von DMPC angepaßt werden. Die Ergebnisse dieser Rechnungen finden sich in Tabelle 7.9.

Eine Anpassung mit völlig freien Parametern liefert allerdings keine sinnvollen Werte für $g$. Hält man jedoch $g=1$ [127] oder $g=0.3$ [144] fest, so ergeben sich für $\gamma a_{k} / a_{m}$ Werte, wie sie auch in der Literatur bekannt sind [143]. Für $g=1$ ist die Funktion (7.20) in Abb. 7.31 als durchgezogenen Kurve dargestellt.

Das hier beschriebene Modell von Cohen und Thunball ist eigentlich "nur" für den fluiden Bereich erdacht, und die Einfachheit des Modells (7.20) läßt sofort erkennen, daß das hier beobachtete Absinken des Diffusionskoeffizienten bei $T_{m}$ im Modell nicht berücksichtigt wird. Aufgrund dessen sollen hier zusätzlich die Ergebnisse aus den Anpassungen von (7.20) nur an die Punkte oberhalb von $T_{m}\left(T_{m}>25^{\circ} \mathrm{C}\right)$ aufgeführt werden. Die Ergebnisse sind in Tabelle 7.10 dargestellt.

\begin{tabular}{|c|c|c|c|}
\hline $\mathrm{g}$ & $\gamma a_{k} / a_{m}$ & $T_{0}\left[{ }^{\circ} \mathrm{C}\right]$ & $D_{0}\left[\mathrm{~cm}^{2} / \mathrm{s}\right]$ \\
\hline \hline $0,001(4)$ & $0,07(22)$ & $21(13)$ & 0 \\
1 & $0,9(2)$ & $-1,3(7.2)$ & 0 \\
0,3 & $0,69(15)$ & $2,5(6.2)$ & 0 \\
\hline
\end{tabular}

Tabelle 7.10: Ergebnisse der Anpassungen an alle Punkte oberhalb von $T_{m}$

\subsubsection{Abschlußbemerkung}

Die Verwendung der Modelle von Eyring und Einstein-Stokes zur Berechnung der Diffusionsvorgänge in Lipidmembranen ist an dieser Stelle sicherlich problematisch, da hinter den hier verdendeten Beziehungen Theorien dreidimensionaler Diffusionsvorgänge stecken. Es ist jedoch eine Anwendung durch die pseudodreidimensionalität der Lipidvesikel denkbar.

Abschließend ist zu sagen, daß es schon recht erstaunlich ist, daß man durch die relativ einfache Berechnung über die Modelle von Eyring [27] und Einstein-Stokes Diffusionskoeffizienten erhält, deren qualitativer Verlauf sinnvoll erscheint, und zudem die Werte in der richtigen Größenordnung liegen. Dieser Sachverhalt kann somit als ein starkes Argument für die Identifikation des tieffrequenten Debye-Terms mit einem Diffusionsprozeß angesehen werden. 


\subsection{Der hochfrequente Debye-Term}

Aus früheren Arbeiten [18] [4] [3] ist bekannt, daß Flüssikeiten, die Moleküle enthalten, welche Kohlenwasserstoffen, wie z.B. Alkohole, Alkane und Alkene, besitzen, eine relativ hochfrequente Einzelzeitrelaxation aufweisen, die auf die sogenannte Kinkenbildung zurückzuführen ist. Die Bildung einer Kinke (Abb. 7.33) führt zu einem leicht höheren Raumbedarf des Moleküls und damit zu einer Dichteveränderung (ca. 25-50 $\AA^{3}$ [150]). Damit ist dieser Effekt sensitiv für die Ultraschallspektroskopie und kann entsprechend detektiert werden. Die auftretenden Konformationsänderungen bilden sich durch eine Drehung einer C-C-Bindung um $120^{\circ}$. Es entsteht eine gauche-Konformation, die jedoch aus sterischen Gründen energetisch sehr ungünstig ist. Folglich bildet sich eine weitere gauche-Konformation des sich anschließenden C-Atoms, so daß eine Situation entsteht, wie in Abb. 7.33 dargestellt. Die so entstandene gauche-trans-gauche-(g-t-g)-Kinke führt zu einer Verkürzung der gesamten Kohlenwasserstoffkette um 1,27 A[12] und wegen des höheren Raumbedarfes der Kinke zu einer lateralen Flächenvergrößerung der Membran.

Aus den Messungen zur Kaloriemetrie und Schallgeschwindigkeit ist bekannt, daß die Lipidkonformationen der Membran im Bereich der Umwandlungstemperatur durch hohe Kooperativität gekennzeichnet sind. Dieses zeigt auch der Verlauf der aus der Bhattacharjee-Ferrell-Theorie errechneten Korrelationslänge (siehe Abb. 7.22). Eine kooperative Bildung der Kinken innerhalb des Kohlenwasserstoffbereiches der Membran ist denkbar. Die Bildung einer Kinke beansprucht mehr Raum und somit könnte die Möglichkeit bestehen, daß durch diesen höheren Raumbedarf benachbarte Lipide ebenfalls zur Bildung einer Kinke angeregt werden. Unter der Annahme einer zur Umwandlungstemperatur langsam steigenden Korrelation benachbarter Molekülkonfi-

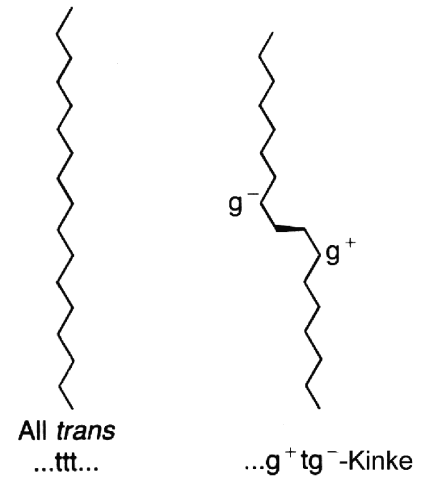

Abb. 7.33: All-trans und g-t-gKonformation, $g^{+}$und $g^{-}$bedeuten Drehung in mathematisch positiver bzw. negativer Richtung gurationen sinkt die entsprechende Relaxationszeit der Kinkenbildung. Diese Überlegungen führen schließlich zu dem Ergebnis, je größer und langlebiger die Domänen auf der Membran sind, desto langsamer und kooperativer ist auch die Kinkenbildung. Dieses Verhalten entspricht jedoch erneut dem Dichte-Fluktuations-Modell von Bhattacharjee und Ferrell. Daher sollte dieser Beitrag zur Ultraschalldämpfung in den schon angepaßten Bhattacharjee-Ferrell-Termen enthalten sein. Die Anpassungsrechnungen von Kapitel 7.2.1 sprechen allerdings gegen diese Argumentation. Nun besteht im weiteren die Möglichkeit, daß die durch die Kinkenbildung verursachten Dichtefluktuationen zu einem zusätzlichen kritischen Beitrag mit einer zu den durch den Schmelzprozeß verursachten Dichtefluktuationen verschiedenen Zeitskalierungen führen. Versuche, die Ultraschallspektren mittels zweier unterschiedlicher Bhattacharjee-Ferrell-Terme zu beschreiben, scheitern jedoch. Dieses ist auch nicht weiter verwunderlich, da man weit oberhalb der Umwandlung keinen kritischen Beitrag mehr erwartet, wohl aber den Beitrag der Kinkenbildung, der allerdings nicht mehr durch die Fluktuationen geprägt ist, sondern eindeutig durch eine Einzel- 


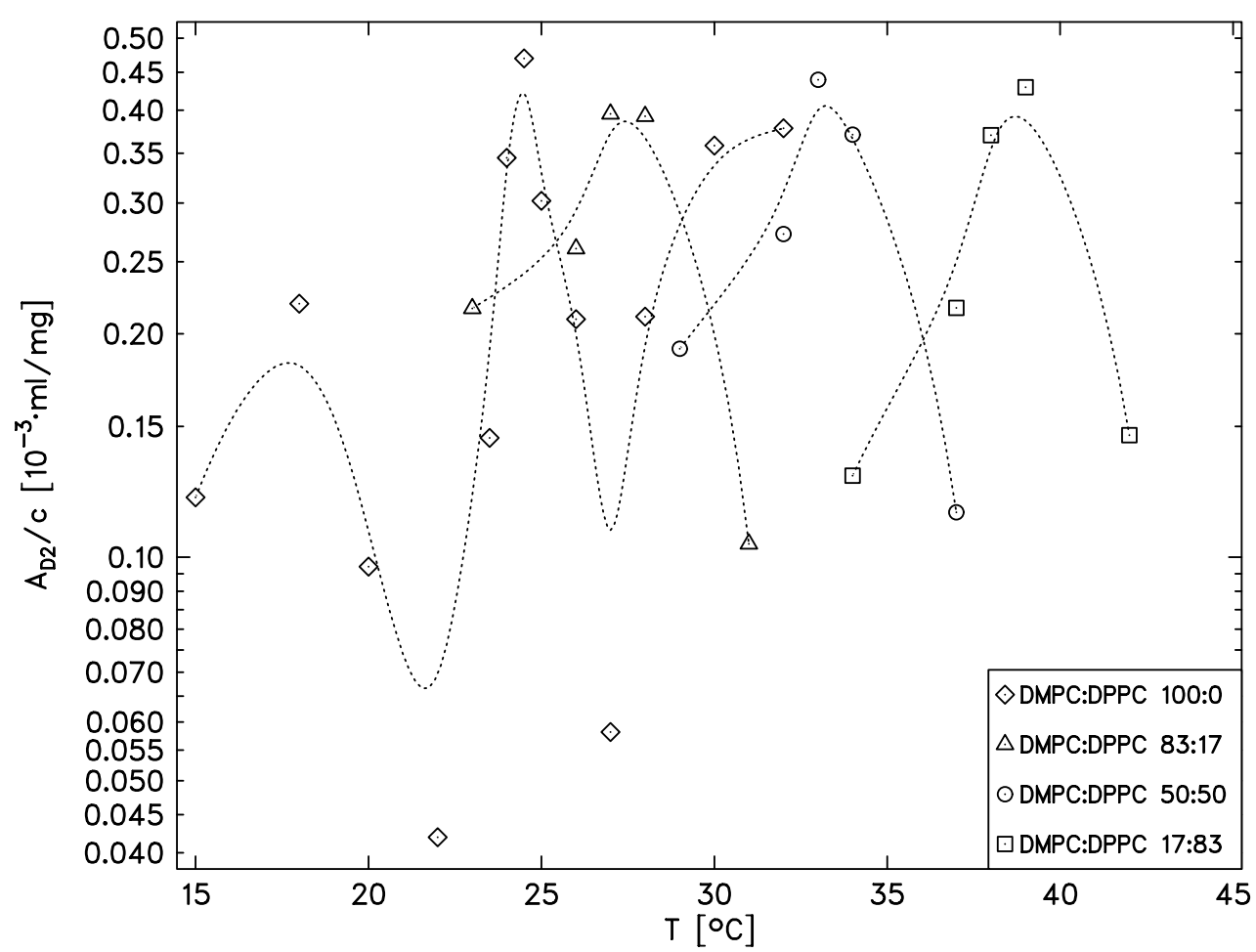

Abb. 7.34: Normierte Amplitude des hochfrequenten Debye-Terms von reinem DMPC und den verschiedenen DMPC/DPPC Mischungen

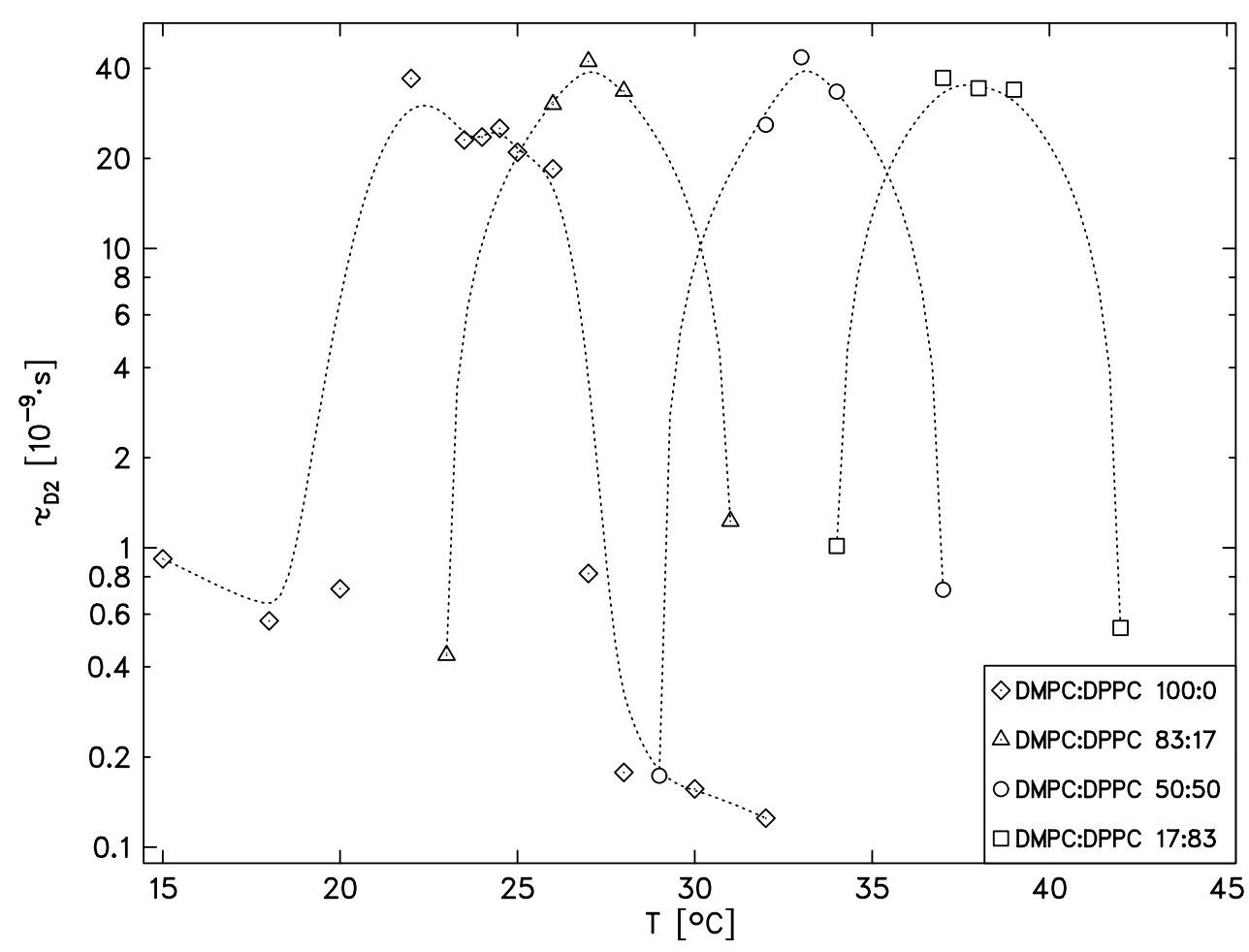

Abb. 7.35: Relaxationszeit des hochfrequenten Debye-Terms von reinem DMPC und den verschiedenen DMPC/DPPC Mischungen 


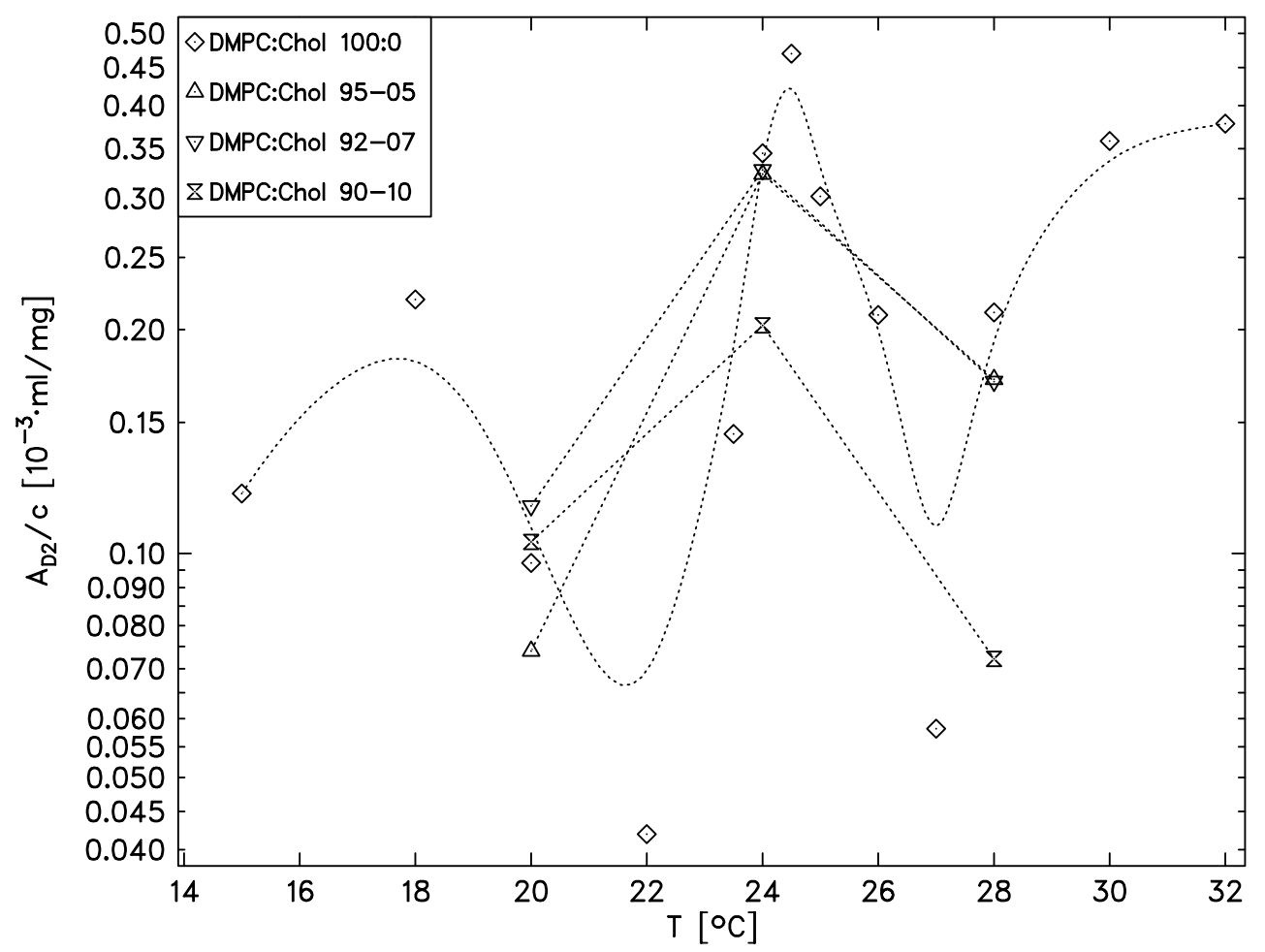

Abb. 7.36: Normierte Amplitude des hochfrequenten Debye-Terms von reinem DMPC und den verschiedenen DMPC/Cholesterin Mischungen

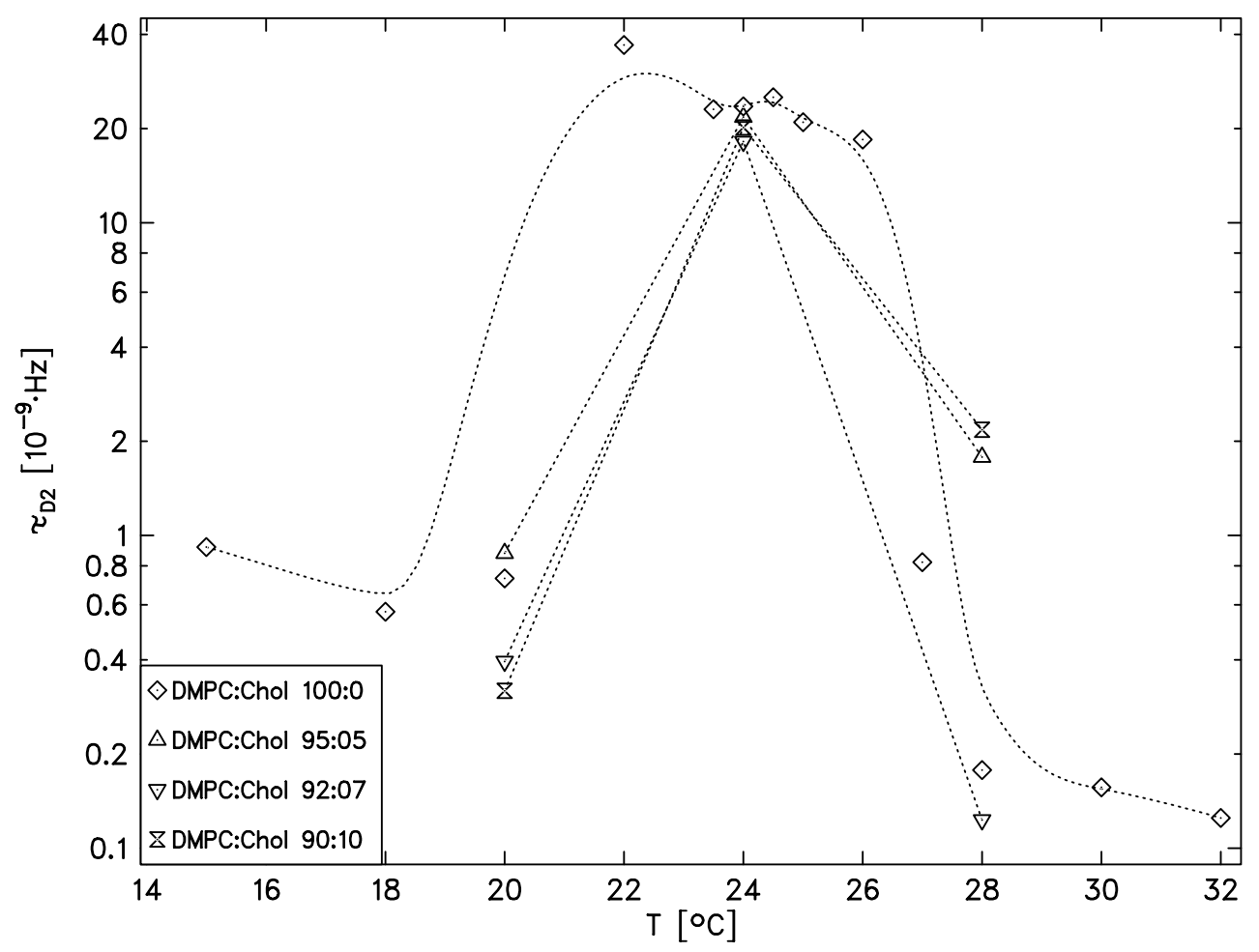

Abb. 7.37: Relaxationszeit des hochfrequenten Debye-Terms von reinem DMPC und den verschiedenen DMPC/Cholesterin Mischungen 
zeitrelaxation beschrieben werden muß, wie er bei den Messungen der Alkohole und Alkane gefunden wird.

Das Auftreten der Fluktuationen während der Phasenumwandlung ist durch den zusätzlichen Hydratationsenergiebeitrag $(\approx 310 \mathrm{cal} / \mathrm{mol}$ bei DPPC [54]) verursacht. Fluide Lipide sind kürzer als gelförmige Moleküle. Der Unterschied in der Dicke der gesamten Membran beträgt etwa $9 \AA[126]$. Durch diesen Längenunterschied entsteht bei einem Molekül ein gewisser Angriffspunkt an den Kohlenwasserstoffketten, der mit dem Wasser wechselwirkt. Da die Kohlenwasserstoffketten jedoch sehr hydrophobe Eigenschaften haben, sind sie bestrebt, sich nicht dem Wasser auszusetzen, was schließlich zur Bildung der Domänen führt. Die Bildung einer Kinke führt zu einer Verkürzung des Moleküls von ca. 1,27 ̊[12]. Somit hat man sich den fluiden Bereich der Membran als eine Ansammlung von Lipiden vorzustellen, bei denen vermehrt Kinken vorhanden sind. Man kann einen erheblich schnelleren Ablauf von Zerfall und Neubildung der Kinken im fluiden Bereich der Membran als im gelförmigen Zustand annehmen. In einem Lipid mit 16 C-Atomen Kettenlänge entstehen und zerfallen oberhalb von $T=T_{c}$ ca. 1-2 Kinken [12] [126].

Im folgenden soll nun versucht werden, den hochfrequenten Debye-Term der Kinkenbildung der Kohlenwasserstoffketten zuzuordnen und mittels der im weiteren dargestellten Theorien zu sinnvollen und konsistenten Ergebnissen zu kommen.

\subsubsection{Dynamisches Verhalten der Rotationsisomerie}

Die charakteristische Frequenz einer Drehung um eine einzelne C-C-Bindung liegt bei ca. $10^{12} \mathrm{~Hz}$ [119] und damit weit oberhalb des hier zugänglichen Frequenzbereiches. Nach Cochran et al. [18] kann jedoch das kooperative Verhalten dieser Kohlenwasserstoffketten zu wesentlich langsameren Relaxationszeiten führen. Es liegt nahe, den möglichen Effekt der Kinkenbildung in den Kohlenwasserstoffketten der Lipide mit Messungen von Alkanen, Alkenen und Alkoholen zu vergleichen [4] [3]. In der Polymerphysik haben sich zwei Modelle zur Beschreibung der Rotationsisomerie etabliert, die von verschiedenen Ansätzen ausgehen.

- Die Polymerketten werden als lineare Kette beschrieben, deren Konformationsänderung entropisch bedingt ist (mittels einer Entropie-Feder), die durch die Viskosität des Lösungsmittels gedämpft wird. Dieses Modell wird von Rouse [124], Bueche und Zimm eingeführt (RB-Modell).

- Die langkettigen Moleküle werden als lineare Ketten von gedämpften Torsionsoszillatoren betrachtet, deren Konformationsänderung durch innere Behinderungen der Rotationen gedämpft werden [141] (DTO-Modell).

\section{RB-Modell}

Das RB-Modell beschreibt die Dynamik einer isolierten Polymerkette durch die Bewegung von gekoppelten, harmonischen Oszillatoren. Das Molekül wird in eine bestimmte Anzahl von Segmenten aufgeteilt, die innerhalb ihrer Grenzen einer gaußschen Statistik gehorchen. Jedes Teilstück verhält sich wie ein elastisches Gummi (entropische Felder), und das gesamte Molekül setzt sich somit aus einer entsprechenden Anzahl von Federn zusammen. Die Anordnung in durch die hydrodynamischen 
Wechselwirkungen mit dem Umgebungsmedium gedämpft. Es ergibt sich folgende Gleichung, die einen Bezug zwischen Viskosität und maximaler Relaxationszeit herstellt:

$$
\tau_{\max }=\frac{\pi \eta_{0} \sigma^{3}}{2 k T \cdot \sin ^{2}\left(\pi /\left(2 z_{g}\right)\right)} .
$$

Dabei ist $\sigma$ der gemittelte Abstand zwischen den Enden einer gaußschen Untereinheit. Liegt nur eine Einheit vor, so ist $\sigma \approx 2 n m$ bei DMPC. $z_{g}$ ist die Anzahl der gaußschen Untereinheiten.

Für die in Gleichung (7.23) auftretende Viskosität gibt es nun zwei Interpretationsansätze. Zum einen ist es möglich, daß die einzelnen Moleküle die makroskopische Viskosität sehen, wie sie über den Diffusionskoeffizienten mittels Einstein-Stokes berechnet werden kann. Zum anderen kann es sein, daß die Moleküle "nur" eine intrinsische Viskosität sehen, die nur durch die Wechselwirkungen der Kohlenstoffketten untereinander verursacht wird. Mittels der Relaxationszeiten des hochfrequenten Debye-Terms wird nun die Viskosität aus Gleichung (7.23) berechnet und in Tabelle 7.11 mit den Viskositäten aus Gleichung (7.18) für die DMPC Suspension verglichen. Dabei wird die Existenz sowohl einer gaußschen Untereinheit angenommen als auch vier Einheiten. Es zeigt sich, daß sich die Werte z.T. erheblich unterscheiden. Insgesamt ist jedoch keinerlei Übereinstimmung mit den zuvor berechneten Viskositäten zu erzielen.

\begin{tabular}{|c|c|c|c|}
\hline $\mathrm{T}\left[{ }^{\circ} \mathrm{C}\right]$ & $\begin{array}{c}\text { Einstein-Stokes } \\
\eta[\mathrm{kg} / \mathrm{m} \cdot \mathrm{s}]\end{array}$ & $\begin{array}{c}\text { RB-Modell } z_{g}=1 \\
\eta[\mathrm{kg} / \mathrm{m} \cdot \mathrm{s}]\end{array}$ & $\begin{array}{c}\text { RB-Modell } z_{g}=4 \\
\eta[\mathrm{kg} / \mathrm{m} \cdot \mathrm{s}]\end{array}$ \\
\hline 15 & $1,13(8)$ & $0,00029(2)$ & $0,0027(2)$ \\
18 & $1,72(19)$ & $0,00018(4)$ & $0,0017(4)$ \\
20 & $0,84(10)$ & $0,00023(2)$ & $0,00219(16)$ \\
22 & $6,4(63)$ & $0,012(12)$ & $0,11(11)$ \\
23,5 & $6,03(66)$ & $0,007508(12)$ & $0,070(12)$ \\
24 & $7,77(46)$ & $0,0076(3)$ & $0,0720(24)$ \\
24,5 & $8,1(21)$ & $0,0082(3)$ & $0,0771(31)$ \\
25 & $3,85(69)$ & $0,0069(5)$ & $0,0643(50)$ \\
26 & $3,8(19)$ & $0,0061(7)$ & $0,0567(65)$ \\
27 & $0,62(4)$ & $0,000270(1)$ & $0,0025(13)$ \\
28 & $0,25(1)$ & $0,000058(2)$ & $0,0006(2)$ \\
30 & $0,21(2)$ & $0,000520(2)$ & $0,0005(2)$ \\
32 & $0,11(1)$ & $0,000418(9)$ & $0,00039(8)$ \\
\hline
\end{tabular}

Tabelle 7.11: Vergleich der Viskositäten des RB-Modells mit den Viskositäten aus Gleichung (7.18)

Die eklatanten Differenzen bei der Viskosität, die unrealistisch kleinen Werte und die Tatsache, daß auch bei [4] dieses Modell zu keinem sinnvollen Ergebnis führte, lassen den Schluß zu, daß das RB-Modell eine unzureichende Beschreibung der Relaxationszeiten liefert. 


\section{DTO-Modell}

Dieses Modell beschreibt die Dynamik der einzelnen Kohlenwasserstoffkette als Überlagerung der Moden einer linearen Anordnung gekoppelter Torsionsoszillatoren:

$$
\tau_{\max }=\frac{1}{2 v_{t} \cdot \sin ^{2}\left(\pi /\left(2 z_{t}\right)\right)} \cdot e^{\Delta G / R T}, \quad z_{t}=\frac{n}{m}
$$

Dabei ist $v_{t}$ die Rotationsfrequenz einer einzelnen C-C-Bindung, $\Delta G$ die molare Aktivierungsenergie und $z_{t}$ das Verhältnis aus Kettenlänge $n$ und der Anzahl $m$ von Kettengliedern eines Torsionsoszillators. Nach Pitzer [119] gelten für Propan die Werte $\Delta G=14.2 \mathrm{~kJ} / \mathrm{mol}$ und $v_{t}=8.49 \cdot 10^{12} \mathrm{~Hz}$. Es ist zunächst nicht eindeutig klar, wie viele Kettenglieder einen Torsionsoszillator bilden. Für DMPC ist $n=14$ sicherlich sinnvoll. Im folgenden wird $v_{t}$ als konstant vorausgesetzt und aus den Relaxationszeiten des hochfrequenten Debye-Terms wird die Aktivierungsenergie ermittelt. Dabei wird für $m$ Werte von 1 bis 3 eingesetzt. Bei $m=3$ ergeben sich sinnvolle Werte, die im Einklang mit Literaturdaten stehen [4] [3] [141] [119].

\begin{tabular}{|c|c||c|c|}
\hline $\mathrm{T}\left[{ }^{\circ} \mathrm{C}\right]$ & $\Delta G[\mathrm{~kJ} / \mathrm{mol}]$ & n-Alkan & $\Delta G[\mathrm{~kJ} / \mathrm{mol}]$ \\
\hline 15 & $17,8(2)$ & n-Hexadekan & $14,9(1)$ \\
18 & $16,8(6)$ & n-Pentadekan & $14,9(2)$ \\
20 & $17,6(2)$ & n-Tetradekan & $14,5(1)$ \\
22 & $27,3(65)$ & n-Dodekan & $14,1(2)$ \\
23,5 & $26,3(4)$ & n-Eikosan & $15,3(4)$ \\
24 & $26,39(8)$ & 7-Tetradekan & $14,3(5)$ \\
24,5 & $26,6(1)$ & 1-Hexanol & $15,9(2)$ \\
25 & $26,2(2)$ & 1-Oktanol & $15,2(6)$ \\
26 & $25,9(3)$ & 1-Dekanol & $14,5(6)$ \\
27 & $18,3(15)$ & 1-Dodekanol & $13,9(3)$ \\
28 & $14,5(11)$ & & \\
30 & $14,3(9)$ & & \\
32 & $13,8(5)$ & & \\
\hline
\end{tabular}

Tabelle 7.12: Vergleich der für $\mathrm{m}=3$ und DMPC errechneten Aktivierungsenergien mit den bekannten Aktivierungsenergien für n-Alkane [4] [3]

Der direkte Vergleich der Werte von fluidem DMPC (Werte für T>27 $7^{\circ}$ ) mit den n-Alkanen zeigt eine sehr gute Übereinstimmung. Auch scheinen die leicht erhöhten Werte für $\Delta G$ bei niedrigen Temperaturen, bei denen die Membran gelförmig ist, sinnvoll.

Zunächst ist es unverständlich, daß die Werte um $T_{m}\left(\approx 24^{\circ} \mathrm{C}\right)$ etwa doppelt so hoch wie im fluiden Bereich liegen. Diese hohen Werte können möglicherweise durch die großen Wechselwirkungsreichweiten erklärt werden. Die Membran erfährt durch das kritische Verhalten und die damit verbundenen Fluktuationen große Änderungen makroskopischer Größen wie Kompressibilität oder Viskosität, was sich in einer relativ hohen Aktivierungsenergie niederschlagen könnte. Stellt man sich anders herum auf den Standpunkt, die Aktivierungsenergie $\Delta G$ auch um $T_{m}$ auf ca. 15 
bis $16 \mathrm{~kJ} / \mathrm{mol}$ festzusetzen, zu müssen zwangsläufig die Parameter $m$ und $v_{t}$ verschieden von den oben angegebenen Werten sein. Läßt man $m=3$ bestehen, so folgt für $v_{t}=8.5 \cdot 10^{10} \mathrm{~Hz}$. Setzt man $m=2$ und erhöht somit die Anzahl der Torsionsoszillatoren und damit auch die möglichen Kinken, so erhält man als realistischen Wert $v_{t}=2.5 \cdot 10^{11} \mathrm{~Hz}$. In jedem Fall ergibt sich ein Absinken der Rotationszeit $v_{t}$ einer einzelnen C-C-Bindung. Dieses kann durchaus im Zusammenhang mit dem Absinken der gemssesenen Relaxationsfrequenz des Debye-Terms gesehen werden. Da sich die Parameter in Gleichung (7.24) für die anderen Lipidmischungen nicht wesentlich ändern, ergeben sich im Rahmen der Fehler die gleichen Aktivierungsenergien wie sie explizit für DMPC in Tabelle 7.12 aufgeführt sind. Es ist jedoch äußerst auffällig, daß sich bei den Suspensionen DMPC/Cholesterin 85:15 und 66:33 kein hochfrequenter Debye-Prozeß ausmachen läßt. Dieses deutet sich schon beim Vergleich der Relaxationsamplitude von DMPC/Cholesterin 90:10 mit denen der Suspensionen mit geringerem Cholesterinanteil an. In der gelförmigen Phase ist die Amplitude gegenüber der 95:05 Mischung stark gefallen und die Relaxationszeit ist relativ kurz. So scheint es wahrscheinlich, daß entweder der Prozeß bei den Mischungen 85:15 und 66:33 zu schwach ausgeprägt ist, als daß er noch erfaßt werden kann, oder außerhalb des Meßfrequenzbereiches liegt. Cholesterin bindet, wie bereits mehrfach erwähnt, durch eine Wasserstoffbrückenbindung an ein Membranlipid. Da aber die Bildung von Kinken zusätzliches Volumen benötigt, Cholesterin jedoch keine Kinken bilden kann und zudem recht dicht an die Kohlenwasserstoffketten bindet (siehe auch Abb. 4.22), behindert das Cholesterin die Bildung von Kinken oder unterbindet sie womöglich vollständig. Diese Vorstellung ist im Einklang mit dem Bild einer fluiden Membran, bei der sich die fluiden Lipide durch eine vermehrte Kinkenbildung auszeichnen [127]. Unter Zugabe von Cholesterin verschwindet zusehends die charakteristische Umwandlung einer Lipidmembran von gelförmig zu fluid, da die Lipide keine Kinken mehr ausbilden können. Im Ultraschallspektrum äußert sich dieses im Verschwinden des hochfrequenten Debye-Term oberhalb von $15 \%$ Cholesteringehalt der Membran.

\subsubsection{Vergleich der Amplitude}

Um neben den Relaxationszeiten auch die Amplitude des hochfrequenten DebyeTerms vergleichen zu können, werden zum Vergleich Meßergebnisse an Alkoholen betrachtet. Im Gegensatz zu n-Alkanen zeichnen sich Alkohole, die ebenfalls den Prozeß der Kinkenbildung aufweisen, durch eine stärkere Wechselwirkung untereinander aufgrund der zusätzlichen Hydroxyl-Gruppe aus und entsprechen somit eher den Vorgängen innerhalb der Lipidmembran. Bei Behrends [4] werden Ultraschallspektren diverser Alkohole behandelt und sollen nun im folgenden mit den hier gemessenen Spektren verglichen werden.

Im Gegensatz zu den Lipidsuspensionen, die immer in wäßriger Lösung vorliegen, wurden die Messungen an den Alkoholen an den reinen Substanzen getätigt. Deshalb muß zunächst die Amplitude auf die Anzahl der Moleküle pro Volumeneinheit normiert werden. Über die Molmasse und die spezifische Dichte kann bei den Alkoholen die Molekülanzahl pro Volumen bestimmt werden. Bei den Lipidsuspensionen reicht die Molmasse und das eingewogene Gewicht aus, um die Anzahl der Moleküle pro Volumen zu bestimmen. Zusätzlich muß die Tatsache beachtet werden, daß die Lipi- 
de überlicherweise zwei kinkenbildende Kohlenstoffketten besitzen $k_{N}=2$, während die Alkohole überlicherweise aus einer Kohlenstoffkette bestehen.

\begin{tabular}{|c|c|c|c|c|}
\hline Bez. & $M_{r}[\mathrm{~g} / \mathrm{mol}]$ & $\rho\left[\mathrm{kg} / \mathrm{m}^{3}\right]$ & $A$ & $A / c[\mathrm{ml} / \mathrm{mol}]$ \\
\hline 1-Hexanol & 102,18 & $818,0(9)$ & $0,110(10)$ & $13,7(12)$ \\
1-Oktanol & 130,23 & $824,0(9)$ & $0,073(50)$ & $11,5(79)$ \\
1-Dekanol & 158,29 & $829,0(9)$ & $0,114(16)$ & $21,8(31)$ \\
1-Dodekanol & 186,33 & $834,0(8)$ & $0,193(18)$ & $43,1(40)$ \\
3-Hexanol & 102,18 & $814,4(8)$ & $0,099(5)$ & $12,4(6)$ \\
2-Methy-1-Pentanol & 102,18 & $817,3(8)$ & $0,108(16)$ & $13,5(20)$ \\
2-Ethyl-1-Hexanol & 130,23 & $828,0(8)$ & $0,159(41)$ & $25,0(64)$ \\
3,7-Dimethyl-1-Octanol & 158,29 & $826,0(8)$ & $0,245(9)$ & $46,9(17)$ \\
\hline
\end{tabular}

Tabelle 7.13: Amplituden und normierte Amplituden der Alkohole und methylierten Alkohole [4]

\begin{tabular}{|c|c||c|c|}
\hline $\mathrm{T}\left[{ }^{\circ} \mathrm{C}\right]$ & $\frac{A}{k_{N} c}\left[\frac{m l}{m o l}\right]$ & $\mathrm{T}\left[{ }^{\circ} \mathrm{C}\right]$ & $\frac{A}{k_{N} c}\left[\frac{m l}{m o l}\right]$ \\
\hline 15 & $41(3)$ & 25 & $102(9)$ \\
18 & $74(15)$ & 26 & $71(8)$ \\
20 & $33(2)$ & 27 & $20(8)$ \\
22 & $14(50)$ & 28 & $72(45)$ \\
23,5 & $49(9)$ & 30 & $121(38)$ \\
24,0 & $117(4)$ & 32 & $128(47)$ \\
24,5 & $159(7)$ & & \\
\hline
\end{tabular}

Tabelle 7.14: Auf die Anzahl der Moleküle und Kohlenwasserstoffketten pro Molekül $k_{N}=2$ normierte Amplituden der DMPC/Wasser Suspension.

Der Vergleich von Tabelle 7.13 und 7.14 zeigt deutlich, daß sich die normierten Amplituden gut entsprechen. Je länger die Kohlenwasserstoffkette bei den unverzweigten Alkoholen ist, desto größer wird auch die Amplitude. Das Ansteigen der Amplitude der DMPC/Wasser Suspension in der Nähe der Umwandlung ist ebenfalls auffällig. Insgesamt kann die relativ gute Übereinstimmung der Amplituden als ein weiteres Indiz für die Identifikation des hochfrequenten Debye-Terms mit der Kinkenbildung betrachtet werden. Bemerkt sein noch, daß die Amplituden der n-Alkane alle etwa eine Größenordnung unter den in Tabelle 7.14 aufgeführten Amplituden liegen. Offensichtlich spielt die Assoziation bzw. die Kooperativität der Moleküle untereinander eine entscheidende Rolle für die Kinkenbildung.

\subsubsection{Anzahl der Kinken pro Alkylkette}

Um eine quantitative Analyse der Relaxationsamplitude $A$ der hochfrequenten Einzelzeitrelaxation (Gleichung (5.10)) zu geben, muß wenigstens einer der Parameter $\Delta V, \Delta H$ oder $\Gamma$ bekannt sein. Bei $\mathrm{H}$. Trauble et al. [142] wird die Volumenzunahme bei reinem Paraffin mit etwa $2 \%$ bis $6 \%$ angegeben. Bei Behrends [4] wird das Reaktionsvolumen, welches durch den Übergang von der all-trans zur cis-Form 
zusätzlich benötigt wird, für die Bildung einer Kinke über die molaren Volumina der $\mathrm{CH}_{2}$-Gruppen berechnet und mit $\Delta V_{0}=1,8 \mathrm{~cm}^{3} / \mathrm{mol}$ angegeben. Der GammaFaktor aus Gleichung (5.11) kann nun mit der Anzahl der cis-trans-Übergänge in Verbindung gebracht werden:

$$
\frac{1}{\Gamma}=\frac{1}{c_{1}}+\frac{1}{c_{2}}
$$

Die Gesamtkonzentration der vorhandenen C-C-Bindungen $c_{g e s}$ ist über die Konzentration der Lipde in der Suspension $c_{\text {lipid }}$ mit der Anzahl der Länge der Kohlenstoffketten $N_{C}$ verknüpft . Dabei muß beachtet werden, daß jedes Lipid zwei Ketten besitzt:

$$
c_{\text {ges }}=2 \cdot N_{C} \cdot c_{\text {lipid }}
$$

Nun läßt sich das im Relaxationsprozeß auftretende Reaktionssvolumen aus kleineren Teilvolumina zusammensetzen, die jeweils die Ursache einer Kinkenbildung sind. Damit ist das resultierende Reaktionsvolumen zunächst durch $\Delta V=k \cdot \Delta V_{0}$ gegeben, wobei $k$ die Anzahl der Kinken pro Kohlenstoffkette ist. Der Anteil der Moleküle mit cis-Bindungen ist entsprechend gegeben durch $n \cdot c_{\text {lipid }}$. Weiter ist die Gesamtanzahl der kinkenbildenden C-C-Bindungen pro Molekül $k=n / N_{C}$. Setzt man nun den Gamma-Faktor $\Gamma$ proportional zur Volumenkonzentration $c_{\text {lipid }}$ mit $\Gamma=\phi \cdot c_{\text {lipid }}$, ergibt sich für die hier eingeführte Proportionalitätskonstante $\phi$ :

$$
\phi=\left(\frac{k}{N_{C}}-\left(\frac{k^{2}}{N_{C}}\right)\right) .
$$

Beachtet man weiterhin, daß der thermische Ausdehnungskoeffizient $\mathcal{A}_{\infty}$ nicht negativ werden darf, so könen im folgenden mittels numerischer Kalkulation die gefragten Größen in Gleichung (5.10) ermittelt werden. Dabei wird fortwährend $\Delta H, \Delta V$ und $\phi$ variiert und das Ergebnis für die Amplitude mit der real gemessenen Amplitude im Rahmen der Fehler verglichen. Der Einfluß der möglichen Reaktionsenthalpie $\Delta H$ auf die Wärmekapazität $C_{p}$ ist dabei vernachlässigbar klein, so daß die Randbedingung $C_{p, \infty}>0$ nicht zum Tragen kommt. Zudem wird für die Berechnungen vorausgesetzt, daß die Dispersion der Schallgeschwindigkeit $c_{s}$ ebenfalls vernachlässigbar klein ist, so daß $c_{s, \infty}=c_{s, 0}$ gesetzt werden kann. Mit der Wärmekapazität $C_{p}=4182$ $\frac{J}{m o l \cdot K}$ und dem thermischen Ausdehnungskoeffizienten $\mathcal{A}_{\infty}=0,00055 \frac{1}{K}$ lassen sich nun die entsprechenden Größen bestimmen. Leider können im Rahmen der Fehler der gemessenen Amplituden die entscheidenen Größen wie Reaktionsenthalpie und Reaktionsvolumen nicht eindeutig bestimmet werden. Im Rahmen von Gleichung (5.10) ergibt sich nur eine Lösungsmenge mit theoretisch unendlich vielen Lösungen. Allerdings ist die Lösungsmenge durch die äußeren Parameter derart eingeschränkt, daß sich als abschließendes Ergebnis ein minimal mögliches Reaktionsvolumen angeben läßt, welches in Tabelle 7.15 für die DMPC/Wasser Suspension für die verschiedenen Temperaturen aufgeführt ist. Auf diese minimal mögliche Amplitude wird im weiteren näher eingegangen.

Da es wahrscheinlicher scheint, daß die Reaktionsenthalpien wesentlich kleiner sind als die Aktivierungsenthalpien (siehe Tabelle 7.12), kann der mögliche Lösungsbereich für die Rekationsvolumina jedoch weiter eingeschränkt werden. Er liegt dann dicht am minimal möglichen Reaktionsvolumen (siehe Abb. 7.38). Dennoch muß 


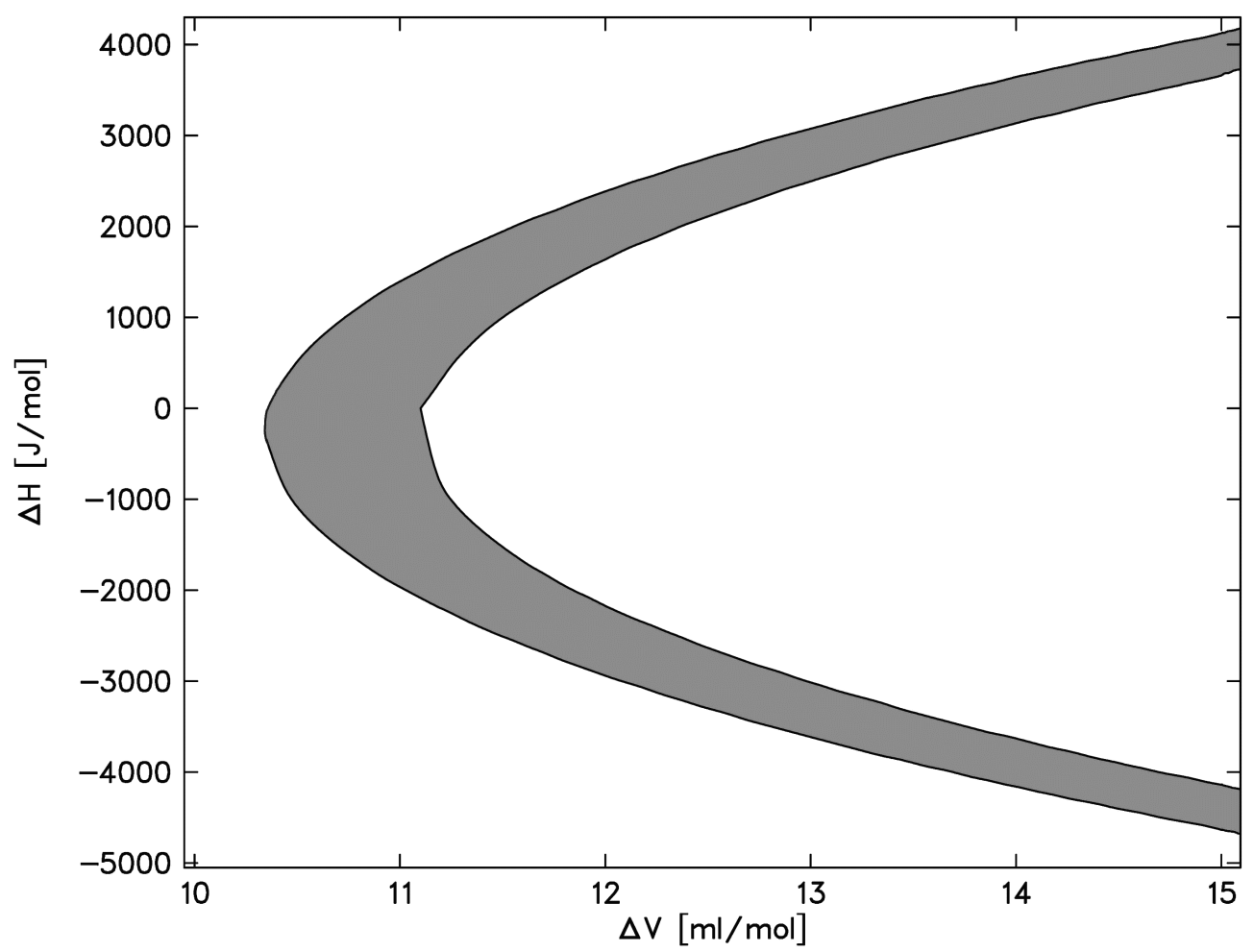

Abb. 7.38: Aus Gleichung (5.10) berechnete Lösungmenge für Reaktionsenthalpie und Reaktionsvolumen der DMPC/Wasser Suspension bei $15^{\circ} \mathrm{C}$

an dieser Stelle auf die prinzipielle Vielfalt der Lösungen in Abb. 7.38 hingewiesen werden.

\begin{tabular}{|c|c||c|c|}
\hline $\mathrm{T}\left[{ }^{\circ} \mathrm{C}\right]$ & $\Delta V_{\text {ges }}\left[\mathrm{cm}^{3} / \mathrm{mol}\right]$ & $\mathrm{T}\left[{ }^{\circ} \mathrm{C}\right]$ & $\Delta V_{\text {ges }}\left[\mathrm{cm}^{3} / \mathrm{mol}\right]$ \\
\hline 15 & $10,76(36)$ & 25 & $17,1(8)$ \\
18 & $14,5(15)$ & 26 & $14,15(75)$ \\
20 & $9,7(3)$ & 27 & $7,4(17)$ \\
22 & $6,2(17)$ & 28 & $13,8(35)$ \\
23,5 & $11,8(12)$ & 30 & $18,3(30)$ \\
24 & $18,25(25)$ & 32 & $18,8(35)$ \\
24,5 & $21,2(4)$ & & \\
\hline
\end{tabular}

Tabelle 7.15: Numerisch berechnete Reaktionsvolumina der DMPC/Wasser Suspensionen.

Man beachte, daß die in Tabelle 7.15 angegebenen Werte auf ein Lipidmolekül bezogen sind. Die sich ergebenden Reaktionsenthalpien entsprechend in etwa denen der Alkohole bei Behrends [4].

Bei Behrends [4] wird ein Vergleich zwischen den n-Alkanen und den wesentlich mehr assoziierenden Alkoholen diskutiert. Die Berechnungen der Reaktionsamplituden für die n-Alkane zeigtn die ständige Bildung von ca. 2 bis 3 Kinken, je nach Anzahl der rotationsfähigen C-C-Bindungen. Während im Rahmen des schon oben diskutierten DTO-Modells die Relaxationszeiten der n-Alkane und Alkohole sehr gut übereinstimmen, zeigen die Alkohole im Gegensatz zu den n-Alkanen eine sehr 
viel größere Relaxationsamplitude. Durch das Vorhandensein einer (OH)-Gruppe bei den Alkoholen, was zur Assoziation dieser Moleküle führt, verhalten sich auch die Alkylketten anders, als es die Ergebnisse der n-Alkane vermuten lassen. Diese Assoziation führt zusammenfassend zu einem zusätzlichen Reaktionsvolumen $\delta V_{Z u s a t z}$ welches die erhöhten Relaxationsamplituden erklären kann. Behrends [4] gibt dieses mit $\delta V_{\text {Zusatz }}=4$ bis $6[\mathrm{ml} / \mathrm{mol}]$ an.

Die in dieser Arbeit betrachteten Lipide zeigen ebenfalls einen hohen Grad der Assoziation bzw. Kooperativität. So ist anzunehmen, daß ähnlich wie bei den Alkoholen auch hier ein zusätzliches Reaktionsvolumen mit einbezogen werden muß. Folglich gilt:

$$
\Delta V_{\text {ges }}=2 \cdot\left(k \cdot \delta V_{0}+\delta V_{\text {Zusatz }}\right) \quad .
$$

Mit $\delta V_{\text {Zusatz }} \approx 5[\mathrm{ml} / \mathrm{mol}]$ und $\delta V_{0}=1,8[\mathrm{ml} / \mathrm{mol}]$ kann so die Anzahl der Kinken pro Alkylkette errechnet werden. Abb. 7.39 zeigt den Verlauf des Parameters $k$ aus Gleichung (7.28) der DMPC/Wasser Suspension über der Temperatur. Der teilweise recht hohen Fehler in der Bestimmung der Amplitude des Debye-Terms führt bei einigen Temperaturen zu recht kleinen Volumina (bei $22^{\circ} \mathrm{C}$ und $27^{\circ} \mathrm{C}$ ). Daraus folgt für den Parameter $k$ bei diesen Temperaturen ein negativer Wert, was jedoch naturgemäß nicht möglich ist. Aufgrund dessen wird der Parameter $k$ bei diesen Temperaturen sehr klein, jedoch größer Null gesetzt. In Abb. 7.39 ist bemerkenswert, daß unterhalb der Umwandlungstemperatur die Anzahl der Kinken pro Alkylkette um einen Wert von etwa 0,5 schwankt, der allerdings oberhalb der Umwandlung bei 2 bis 3 liegt. Dieser Werteverlauf entspricht genau den Werten aus der Literatur: Sackmann [127] gibt ebenfalls für den fluiden Zustand der Membran eine Kinkenbildung von ca. 2 pro Alkylkette an. Dieses bestätigt den Schmelzvorgang der Membran, der vor allem durch die Bildung von Kinken und die schnelle Rotation der C-C-Bindungen beschrieben wird. In Abb. 7.39 ist ebenfalls zu sehen, daß genau in der Umwandlung das hier berechnete k relativ groß ist. Höchstwahrscheinlich ist aber auch das zusätzliche Reaktionsvolumen $\delta V_{Z u s a t z}$ in der Umwandlung bedingt durch die hohen Wechselwirkungsreichweiten erhöht, wird aber bei den Berechnungen als konstant über der Temperatur angenommen.

Abb. 7.40 zeigt den Parameter $k$ als Funktion der Cholesterinkonzentration in der Membran in der fluiden Phase. Es ist ein deutliches Absinken der Kinkenanzahl pro Alkylkette mit steigendem Cholesteringehalt zu sehen. Dieses scheint die oben diskutierte Annahme zu bestätigen, daß das Cholesterinmolekül relativ stark über eine Wasserstoffbrückenbindung an das DMPC Lipid bindet und durch seine hohe Steifigkeit die schnellen Rotationsisomerien und Kinkenbildungen unterbindet. Über ca. $10 \%$ Cholesteringehalt in der Membran bildet sich eine cholesterinreiche Phase, die einen relativ festen Cholesteringehalt besitzt. Diese Phase vermindert zum einen die üblichen Fluktuationen der reinen Lipidphase, und zum anderen beeinflußt das Cholesterin die cholesterinreiche Phase. Cholesterin besteht im Gegensatz zu den hier behandelten Lipiden hauptsächlich aus fünf Kohlenstoffringen. Diese weisen naturgemäß keine Rotationsisomerie wie die Alkylketten der Lipide auf. Durch diese Unfähigkeit der Kinkenbildung unterbindet das Cholesterin die Kooperativität bzw. Wechselwirkung im Bezug auf die Kinkenbildung zwischen den Lipidmolekülen. Zusätzlich wird die Kinkenbildung der Lipide selbst größtenteils unterbunden, da durch die Anwesenheit des Cholesterins kein entsprechend freies 


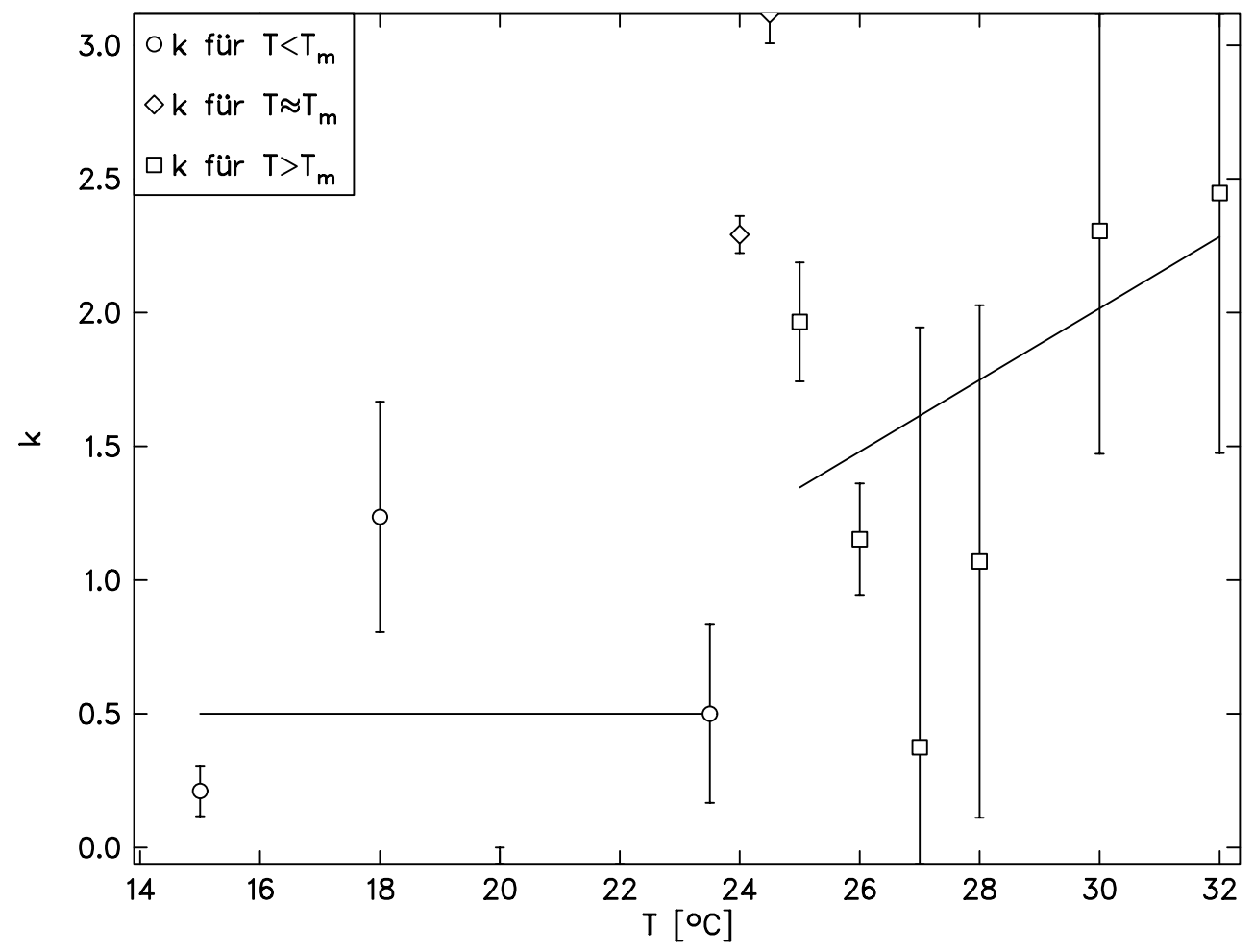

Abb. 7.39: Mit Gleichung (7.28) berechnete Anzahl der Kinken $k$ pro Alkylkette der DMPC/Wasser Suspension

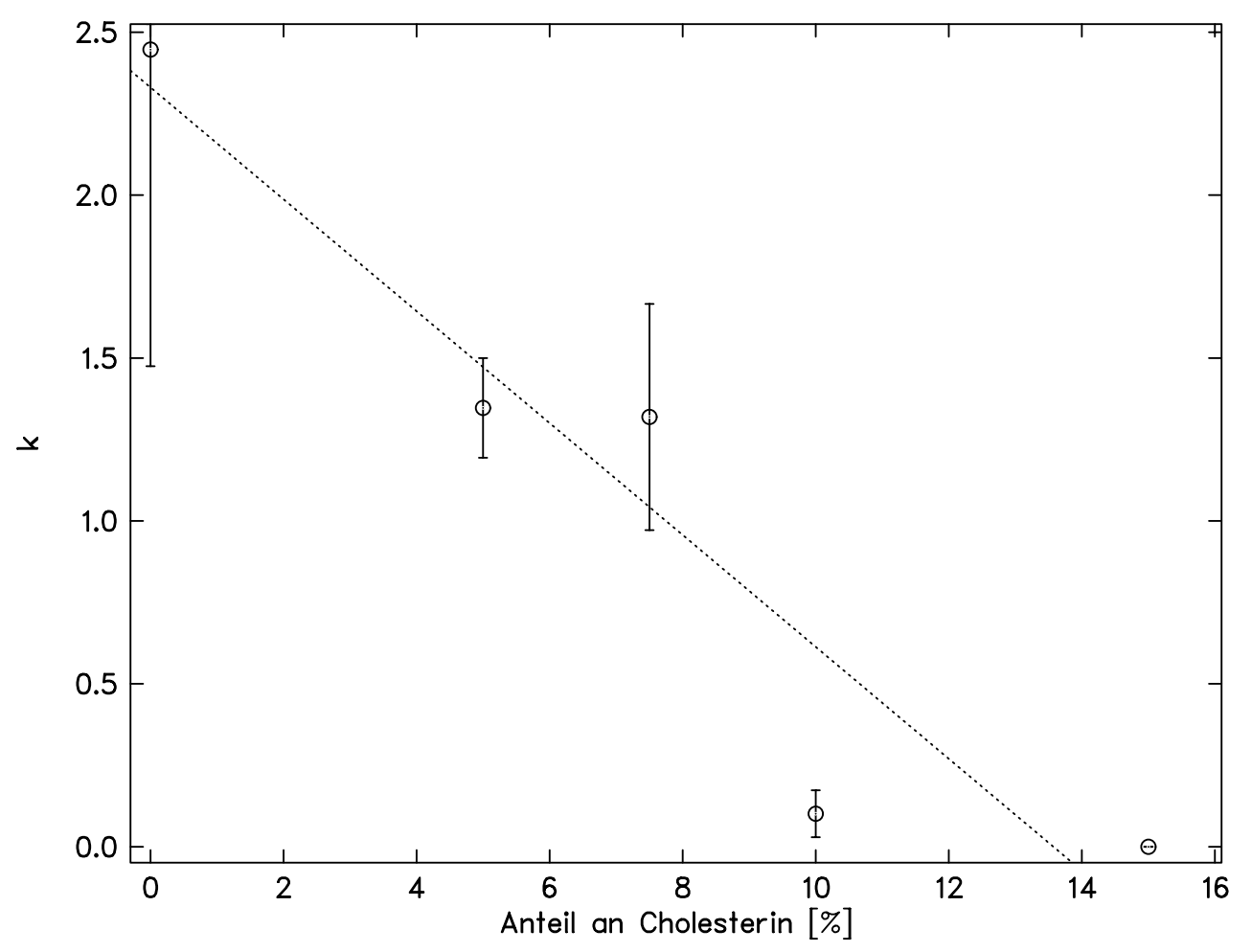

Abb. 7.40: Mit Gleichung (7.28) berechnete Anzahl der Kinken $k$ pro Alkylkette der DMPC/Cholesterin Suspensionen allerdings nur in der fluiden Phase 
Volumen zur Verfügung steht. So ist schon bei den Spektren der DMPC/Cholesterin 85:15 Suspension kein hochfrequenter Debye-Term mehr in den Spektren enthalten.

\subsection{Der B-Wert}

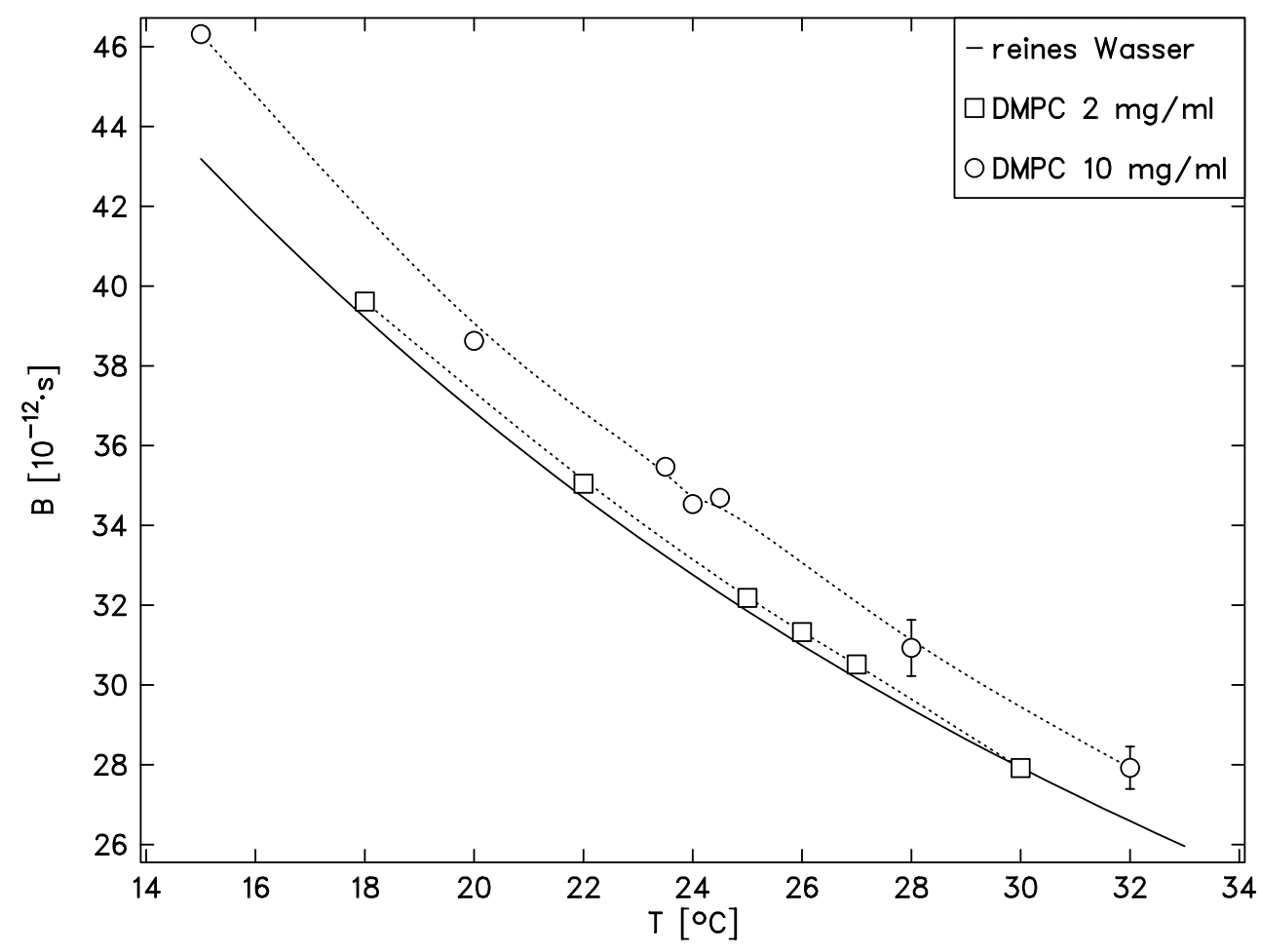

Abb. 7.41: Vergleich der $B$-Werte aus dem Spektren der DMPC/Wasser Suspensionen mit unterschiedlicher Konzentration

Alle Relaxationsprozesse, die sich oberhalb des meßbaren Frequenzbereiches anschließen, werden in den Ampaßrechnungen in dem $B$-Wert zusammengefaßt (siehe Kap. 5). Aufgrund des hohen Wasseranteils in den hier betrachteten Suspensionen ist ein ähnlicher Werteverlauf wie bei reinem Wasser zu erwarten. Abb. 7.41 zeigt den Verlauf der $B$-Wertes von Wasser über der Temperatur im Vergleich zu den DMPC/Wasser Suspensionen. Da diese jedoch in unterschiedlicher Konzentration gemessen wurden, ergeben sich hier zwei unterschiedliche Kurven. Aufgrund des höheren Volumenanteils von DMPC bei den Suspensionen mit $10 \frac{\mathrm{mg}}{\mathrm{ml}}$ liegen die $B$ Werte entsprechend höher als bei den Suspensionen mit $2 \frac{\mathrm{mg}}{\mathrm{ml}}$. Die hochfrequenten Anteile in den Spektren von Alkanen, Alkenen und Alkoholen liegen bei etwa 100 ps (bei $25{ }^{\circ} \mathrm{C}$ ), so daß die Tendenz zu höheren $B$-Werten bei steigendem Lipidgehalt qualatitativ zu erklären ist. 


\section{Zusammenfassung}

In dieser Arbeit wurden Lipidmembranen im Hinblick auf ihr Phasenverhalten und die beim Phasenübergang auftretenden Fluktuationen untersucht. Desweiteren standen Relaxationsphänomene und deren molekulare Ursachen im Vordergrund. Dabei wurden verschiedenste Membranzusammensetzungen untersucht, um einzelne molekulare Vorgänge möglichst sicher identifizieren zu können.

Mittels Kalorimetrie und Schallgeschwindigkeitsmessung konnten Schmelzvorgänge und Phasenübergänge in den jeweiligen Membranen nachgewiesen werden. DMPC/DPPC Membranen erwiesen sich als ideale binäre Mischung und zeigten aufgrund dessen eine charakteristische Umwandlungstemperatur in Abhängigkeit ihrer Zusammensetzung. Gleiches gilt für DLPC/DMPC Membranen, die sich im Temperaturbereich unterhalb der DMPC/DPPC Mischungen anschließen. Jedoch mischen sich DLPC/DPPC Membranen nicht mehr ideal. Sie zeigen zwei mehr oder wenig ausgeprägte Umwandlungstemperaturen in Abhängigkeit ihrer Zusammensetzung. In DLPC/DPPC Membranen bilden sich entmischte Bereiche, die nur aus Lipiden einer Sorte bestehen und ihren individuellen Schmelzpunkt besitzen. Ähnlich verhalten sich auch DMPC/DMG Membranen. Jedoch bildet sich hier bei Konzentrationen über ca. $25 \%$ DMG relativ schnell eine DMG-reiche Phase mit ihrer eigenen Umwandlungstemperatur. So zeigt auch die DMPC/DMG 75:25 Suspension zwei ausgeprägte Schmelzpunkte. Mischt man DMPC mit Cholesterin, so zeigt sich eine charakteristische Verbreiterung des Phasenumwandlunggeschehens. Cholesterin bindet über eine Wasserstoffbrückenbindung an ein DMPC Molekül und unterbindet so die Kooperativität zwischen den Lipiden. Zusätzlich bildet sich ab ca. 7\% Anteil eine cholesterinreiche Phase, die weitestgehends nicht an den Fluktuationen teilnimmt und somit auch die Fluktuationen in der nicht cholesterinreichen Phase unterbindet. Mit Hilfe der Theorie von T. Heimburg konnten die beobachteten Schmelzvorgänge auf Fluktuationen der Enthalpie und Dichte zurückgeführt werden. In der Umwandlung bilden sich Domänen aus gelförmigen und fluiden Lipiden, die nebeneinander koexistieren und ständig ihre Größe und Form verändern. Zusätzlich konnten die aufgenommenen Wärmekapazitätskurven mit den Schallgeschwindigkeitskurven in Verbindung gebracht werden. Die gute Übereinstimmung der aus der Theorie berechneten Kurven mit den gemesssenen Kurven läßt auf die Korrektheit der Annahmen in dem theoretischen Modell schließen. Die Membran scheint bei den verwendeten Frequenzen um $2 \mathrm{MHz}$ adiabatisch vom umgebenden Wasser entkoppelt zu sein, so daß die bei Kompression entstehende Wärme in der Membran zu verbleiben scheint. Untersuchungsgegenstand in der dielektrischen Spektroskopie waren die molekularen Vorgänge an der Grenzfläche zwischen Membran und Wasser. Speziell wurden die Relaxationsvorgänge der zwitterionischen Kopfgruppen ausgemessen. Diese konnten zweifelsfrei im Frequenzbereich um $80 \mathrm{MHz}$ nachgewiesen werden und es zeigte sich, daß die Schmelzvorgänge in den Membranen Auswirkungen auf die Bewegungen der Kopfgruppen haben. Mittels der Theorie von R. Pottel war es möglich, die gemessenen Spektren mit den entscheidenden Größen Orientierungskorrelationsfaktor und Beweglichkeit der Kopfgruppen in Verbindung zu bringen. Membranen aus reinem DMPC zeigen einen sprunghaften Anstieg der Beweglichkeit der Kopfgruppen oberhalb von $T_{c}$. Dieses war analog bei der DMPC/DPPC Mischung zu beobachten. In der Nähe der Umwandlungstemperatur liegt eine leicht erhöhte Korrelation der Kopfgruppen untereinander vor. Auch die DMPC/DMG Suspension paßt in dieses 
Bild. Diese Mischung zeigt zudem, wie aus den kalorimetrischen Messungen zu erwarten, zwei Maxima des Korrelationsfaktors. Dennoch bleibt festzuhalten, daß das Auftreten von Domänen und Fluktuationen nur einen relativ geringen Einfluß auf die Kopfgruppenbewegung zu haben scheint. Die Beimischung von Cholesterin in DMPC Membranen brachte keine merkliche Veränderung in der Beweglichkeit der Kopfgruppen. Es zeigte sich ein Abnehmen der Orientierungskorrelation mit steigendem Cholesteringehalt. Cholesterin bindet über eine Wasserstoffbrückenbindung an das Glyceringerüst eines DMPC-Moleküls und kann so keinen merklichen Einfluß auf die Beweglichkeit der Kopfgruppe ausüben. Dennoch wird die Wechselwirkung zwischen den Kopfgruppen niedriger, da durch die zusätzlichen Cholesterinmoleküle in der Membran auch die Abstände zwischen den DMPC Molekülen zunehmen.

Die Ultraschallspektroskopie diente einerseits dazu, die Natur der Fluktuationen zu erforschen, andererseits konnten zusätzliche molekulare Vorgänge in den Membranen beobachtet werden. Insgesamt wurden 26 Spektren von $300 \mathrm{kHz}$ bis $2 \mathrm{GHz}$ aufgenommen und aus anderen Arbeiten zusätzlich 21 Spektren einer eingehenden Untersuchung unterzogen.

Es zeigte sich, daß die Theorie von Bhattacharjee und Ferrell den kritischen Beitrag gut beschreiben konnte. Der kritische Beitrag zur Ultraschalldämpfung konnte mit den Zustandsfluktuationen der Lipide im Bezug auf Dichte und Enthalpie erklärt werden. Jedoch liegen den Fluktuationen dabei keine Molekülbewegungen zugrunde, sondern es handelt sich bei der Diffusion um eine Zustandsdiffusion. Die Spektren der reinen DMPC Suspension und der DMPC/DPPC Suspensionen zeigten in der Nähe der Umwandlung ein charakteristisches Absinken der charakteristischen Relaxationsrate $\omega_{B F}$ und damit eine Verlangsamung der Dynamik. Während $\omega_{B F}$ bei $T_{m}$ gegen Null strebt, steigt die Amplitude $A_{B F}$ gegen ein endliches Maximum. Der Verlauf von $\omega_{B F}$ über der Temperatur kann mittels eines Potenzgesetzes mit den entsprechenden kritischen Exponenten beschrieben werden. Die Korrelationslängen liegen unter Verwendung von $D_{\text {gel }} \approx 3 \cdot 10^{-11} \frac{\mathrm{cm}^{2}}{\mathrm{~s}}$ und $D_{\text {fluid }} \approx 7 \cdot 10^{-9} \frac{\mathrm{cm}^{2}}{\mathrm{~s}}$ unterhalb von $T_{m}$ im Bereich von ein bis zwei Moleküllängen und steigen oberhalb von $T_{m}$ auf ca. 35 Moleküllängen an. Bei $T_{m}$ strebt die Korrelationslänge gegen unendlich. Die DMPC/Cholesterin Suspensionen zeigen ein Absinken der Korrelationslänge mit steigendem Cholesteringehalt. Dieses steht im Einklang mit den Ergebnissen aus den Wärmekapazitäts- und Schallgeschwindigkeitsmessungen. Zum ersten Mal konnte ein kritisches System von beiden Seiten zu $T_{m}$ hin betrachtet werden. Die Messung der Skalierungsfunktion sowohl oberhalb, als auch unterhalb der kritischen Temperatur zeigt dies eindrucksvoll. Aus dem Temperaturverlauf von $\omega_{B F}$ konnte der kritische Exponent $z \nu$ auf 1.73(13) für $T<T_{m}$ und 1.92(38) für $T>T_{m}$ festgesetzt werden. Die gute Übereinstimmung mit dem theoretischen Wert $z \nu=1.93$ deutet ebenfalls auf die Anwendbarkeit des Modells von Bhattacharjee und Ferrell auf die Lipidsuspensionen hin - zumindest für das zeitliche Verhalten der Fluktuationen. Die Tatsache, daß einerseits die charakteristische Relaxationsrate $\omega_{B F}$ bei $T_{m}$ gegen Null strebt und sich der Verlauf dieser Größe über der Temperatur sehr gut mit den Potenzgesetzen beschreiben läßt, andererseits die Amplitude $A_{B F}$ gegen ein endliches Maximum bei $T_{m}$ strebt, muß als Hinweis auf die Endlichkeit bzw. die Besonderheit der binären Flüssigkeit aus Lipid und Wasser gedeutet werden.

Zusätzlich zu dem kritischen Anteil lagen bei fast allen Spektren zwei zusätzliche Einzelzeitrelaxationen vor. Eine Außnahme bilden nur die Spektren der 
DMPC/Cholesterin 85:15 und 66:33 Suspensionen.

Der tieffrequente Debye-Term konnte mittels des freien Volumenmodells über das Modell von Eyring und Einstein-Stokes der realen Massediffusion zugeschrieben werden. Die ermittelten Diffusionskoeffizienten liegen bei der reinen DMPC Suspension unterhalb von $T_{m}$ bei $D_{\text {gel }} \approx 1 \cdot 10^{-10} \mathrm{~cm}^{2} / \mathrm{s}$ und oberhalb von $T_{m}$ bei $D_{\text {fluid }} \approx 1 \cdot 10^{-8} \mathrm{~cm}^{2} / \mathrm{s}$. In der Nähe der Umwandlungstemperatur zeigt der Verlauf der Diffusionskoeffizienten ein leichtes Absinken bedingt durch die Verlangsamung der Relaxationszeit des Prozesses. Offensichtlich scheint die Bildung von Domänen und damit die Bildung von Phasengrenzen zwischen den Domänen, die Diffusion der einzelnen Lipide zu behindern. Der Verlauf des Diffusionskoeffizienten über der Temperatur konnte mit Hilfe des Modells von Cohen und Thunball verifiziert werden und entspricht einem exponentiellen Gesetz.

Der hochfrequente Debye-Term konnte mit der Rotationsisomerie der Alkylketten der Lipide identifiziert werden. Die Lipidmoleküle führen in der fluiden Phase - im Gegensatz zur relativ starren gel-förmigen Phase - schnelle Rotationsbewegungen um die C-C Bindungen aus und bilden dabei Kinken, was zusätzliches Volumen benötigt. Mittels des DTO-Modells gelang ein Vergleich der Aktivierungsenergien von DMPC $\Delta G \approx 16 \mathrm{~kJ} / \mathrm{mol}$ mit den Werte von n-Alkanen und Alkoholen, bei denen ebenfalls Kinkenbildung vorliegt. Der Vergleich der Amplitude des hochfrequenten Debye-Terms und die sehr gute Übereinstimmung der entsprechenden Amplituden deutet auf die Ähnlichkeit der Vorgänge in den Membranen mit den Relaxationsprozessen in assoziierenden Alkoholen hin. Schließlich gelang mit Hilfe von numerischer Berechnung die Bestimmung des mimimal möglichen Reaktionsvolumens pro Alkylkette. Unter Einbeziehung der speziellen Eigenschaften der assoziierenden Alkohole, die durch die (OH)-Gruppe einen zusätzlichen Beitrag zum Reaktionsvolumen aufweisen, und einer analogen Übertragung dieser Eingenschaften konnte die Anzahl der Kinken pro Alkylkette in Abhängigkeit von der Temperatur ermittelt werden, die unterhalb von $T_{c}$ bei $k \approx 0,5$ und oberhalb $k \approx 2$ liegt. 


\section{Literaturverzeichnis}

[1] P. F. F. Ameida, W. L. C. Waz, Lateral Diffusion in Membranes, Handbook of Biological Physics, Editors: R. Lipowsky, E. Sackmann, Elsevier Science B.V. 1 (1995) 305 ff.

[2] F. H. Anthony, E. Freire, Analytical Biochemistry 116 (1981) 116-167

[3] R. Behrends, Diplomarbeit, Mat.-Nat-Fak. Univ. Göttingen (1995)

[4] R. Behrends, Dissertation, Mat.-Nat.-Fak. Univ. Göttingen (1999)

[5] L. Beney, E. Linares, E. Ferret, P. Gervias, Eur. Biophys. J. 27 (1998) 567-574

[6] L. Bergmann, Der Ultraschall, S. Hirzel Verlag Suttgart (1954)

[7] A. B. Bhatia, Ultrasonic Absorption, Oxford At The Clarendon Press (1967)

[8] J. K. Bhattacharjee, persönliche Mitteilung

[9] J. K. Bhattacharjee, R. A. Ferrell, Physical Review A 24 (1981) 3

[10] J. K. Bhattacharjee, R. A. Ferrell, Physics A 250 (1985) 83-90

[11] J. K. Bhattacharjee, R. A. Ferrell, Physical Review E 56 (1997) 5

[12] S. Blasenbrey, W. Pechold, Ber. Bunsenges. physik. Chem. 74 (1970) 784

[13] A. Blume, Biochemistry 22 (1983) 5436-5442

[14] H. E. Bömmel, K. Dransfeld, Phys. Rev. Lett. 1 (1958) 234

[15] C. J. F. Böttcher, P. Bordewijk, Theory Of Electric Polarisation, Elsevier Scientific Publishing Company, Amsterdam (1978)

[16] G. Cevc, D. Marsh, Phospholipid bilayers, In: E. E. Bittar (Herausgeber): 5 der Reihe Cell biology: a series of monographs, John Wiley and Sons, New York 1. Auflage (1987)

[17] R. J. Clarke, Biochimica et Biophysica Acta 1327 (1997) 269-278

[18] M. A. Cochran, P. B. Jones, A. M. North, R. A. Pethrick, 68 (1972) 1719

[19] M. H. Cohen, D. Thurnbull, The Journal of Chemical Physics 31 (1959) 5

[20] F. Dumas, J.-F. Tocanne, G. Leblanc, M.-C. Lebrun, Biochemistry 39 (2000) $4846-4854$

[21] F. Dumas, M.-C. Lebrun, J.-F. Tocanne, FEBS Letters 458 (1999) 271-277

[22] R. V. Durvasula, Ching-hsien Huang, Biochimica et Biophysica Acta 1417 (1999) 111-121

[23] F. Eggers, Th. Funck, Rev. Sci. Instrum 44 (1973) 969

[24] F. Eggers, U. Kaate, Meas. Sci. Technol. 7 (1996) 1-19

[25] F. Eggers, U. Kaatze, K. H. Richmann, T. Telgmann, Meas. Sci. Technol. 5 (1994) 1131-1138 
[26] H.-B. Engelbert, R. Lawaczeck, Ber. Bunsenges. Phys. Chem., 89 (1985) 754759

[27] H. Eyring, J. Chem. Phys., 4 (1936) 283

[28] R. A. Ferrell, J. K. Bhattacharjee, Physics Letters 86A (1981) 2

[29] R. A. Ferrell, J. K. Bhattacharjee, Physical Review A 31 (1985) 3

[30] J. B. Finean, Chem. Phys. Lipids 54 (1990) 147-156

[31] M. E. Fisher, Rep. Prog. Phys. 30 (1967) 615

[32] M. Fixman, Advan. Chem. Phys. 4 (1962) 175

[33] M. Fixman, J. Chem. Phys. 36 (1962) 1965

[34] M. Fixman, J. Chem. Phys. 47 (1962) 2807

[35] T. Fliessbach, Elektrodynamik, BI-Wiss.-Verl., (1994) 303, 322-325

[36] R. Folk, G. Moser, Europhysics Letters 412 (1998) 177-182

[37] R. Folk, G. Moser, Physical Review E 58 (1998) 5

[38] I. Fournier, J. Barwicz, P. Tancréde, Biochimica et Biophysica Acta 1373 (1998) $76-86$

[39] H. Fröhlich, Theory Of Dielectrics, Oxford University Press (1958) 70-77

[40] R. C. Gamble, P. R. Schimmel, Proc. Natl. Acad. Sci. USA 75 (1978) 3011-3014

[41] P. Garidel, A. Blume, Biochem. Biophys. Acta 1371 (1998) 83-95

[42] P. Garidel, C. Johann, L. Mennicke, A. Blume, Eur Biophys J, 27 (1997) 447459

[43] A. Genz, J. F. Holzwarth, T. Y. Tsong, Biophys. J. 50 (1986) 1043-1051

[44] R. Goetz, R. Lipowsky, Journal of Chemical Physics 17 (1998) 108

[45] C. Grosse, Consejo Nacional de Investigaciones Cientificas y Técnicas (1996)

[46] K.-D. Göpel, Dissertation, Mat.-Nat.-Fak. Univ. Göttingen (1982)

[47] O. Göttmann, U. Kaatze, P. Petong, Meas. Sci. Technol. 7 (1996) 525-534

[48] S. Halstenberg, Diplomarbeit, Mat.-Nat.-Fak. Univ. Göttingen (1997)

[49] S. Halstenberg, T. Heimburg, T. Hianik, U. Kaatze, R. Krivanek, Biophys. J. 75 (1998) 264-271

[50] G. G. Hammes, P. B. Roberts, Biochimica et Biophysica Acta 203 (1970) 220227

[51] J. E. Harkness, R. D. White, Biochimica et Biophysica Acta 552 (1979) 450-456

[52] J. N. Hasted, Aqueous Dielectrics, Chapman and Hall, London (1973), 20-23

[53] T. Heimburg, Biocimica et Biophysica Acta, 1415 (1998) 147-162

[54] T. Heimburg, R. L. Biltonen, Biophys J. 70 (1996) 84-96 
[55] T. Heimburg, R. L. Biltonen, Biochemistry 33 (1994) 9477-9488

[56] T. Heimburg, U. Würz, D. Marsh, Biophys. J. 63 (1992) 1369-1378

[57] R. Henze, Dissertation, Mat.-Nat.-Fak. Univ. Göttingen (1978)

[58] K. Herzfeld, T. Litovitz, Absorption and Dispersion of Ultrasonic Waves, Academic Press New York and London (1959)

[59] T. Hianik, S. Küpcü, U.B. Sleytr, P. Rybár, R. Krivánek, U. Kaatze, Colloids and Surfaces A: Physiochemical and Engineering Aspects 147 (1999) 331-339

[60] T. Hianik, U. Kaatze, D. F. Sargent, R. Krivánek, S. Halstenberg, W. Pieper, J. Gaburjaková, M. Gaburjaková, M. Pooga, U. Langel, Bioelectrochem. Bioenerg. 42 (1997) 123-132

[61] P. C. Hohenberg, B. I. Halperin, Rev. Mod. Phys. 435 (1997) 49

[62] B. I. Holperin, P. C. Hohenberg, Phys. Rev. 177 (1969) 952

[63] J. Huang, J. T. Buboltz, G. W. Feigenson, Biochimica et Biophysica Acta 1417 (1999) 89-100

[64] D. G. Hunter, B. J. Frisken, Biophys. J. 74 (1998) 2996-3002

[65] M. Höckel, Diplomarbeit, Mat.-Nat.-Fak. Univ. Göttingen (1999)

[66] J. H. Ipsen, Ole G. Mouritsen, Martin J. Zuckermann, Biophys. J. 56 (1989) 661-667

[67] V. P. Ivanova, Dissertation, Mat.-Nat.-Fak. Univ. Göttingen (2000)

[68] K. Jørgensen, O. G. Mouritsen, Thermochimica Acta 328 (1999) 81-89

[69] M. Janiak, D. M. Small, G. G. Shipley, J. Biolog. Chem. 13 (1979) 6068-6078

[70] A. J. Jin, D. Huster, K. Gawrisch, R. Nossal, Eur. Biophys. J. 28 (1999) 187-199

[71] U. Kaatze, A. Dittrich, K.-D. Göpel, R. Pottel, Chemistry and Physics of Lipids, 35 (1984) 279-290

[72] U. Kaatze, B. Wehrmann, R. Pottel, J. Phys. E: Rev. Sci. Instrum 20 (1987) 1015

[73] U. Kaatze, J. Chem. Eng. Data 34 (1989) 371-374

[74] U. Kaatze, J. Sol. Chem Vol. 11 (1997) 26

[75] U. Kaatze, K. Giese, J.Phys.E.: Sci.Instrum., 13 (1980) 133-141

[76] U. Kaatze, K. Lautscham, Meas. Sci. Technol. 21 (1988) 402

[77] U. Kaatze, K. Lautscham, M. Brai, J. Phys. E: Sci. Instrum. 21 (1988) 98

[78] U. Kaatze, K. Lautscham, Biophysical Chemistry 32 (1988) 153-160

[79] U. Kaatze, K.-D. Göpel, R. Pottel, J. Phys. Chem, 89 (1985) 2565-2571

[80] U. Kaatze, Phys. Med. Biol., 35 (1990) 12 1663-1681

[81] U. Kaatze, V. Kühnel, G. Weiss, Ultrasonics 34 (1996) 51-58 
[82] U. Kaatze, V. Kühnel, K. Menzel, S. Schwerdtfeger, Meas. Sci. Technol. 4 (1993) 1257

[83] U. Kaatze, Dielektrische Relaxation, Beitrag zur Sommerschule "Physik des flüssigen Zustandes", St. Georgen/Längsee/Kärnten, (12. bis 23.9.1977), 3, 4752

[84] L. P. Kadanoff, J. Swift, Phys. Rev. E 166 (1968) 89

[85] M. Kahle, Diplomarbeit, Mat.-Nat.-Fak. Univ. Göttingen (2000)

[86] C. Karolis, Hans G. L. Coster, Terry C. Chilcott, Kevin D. Barrow, Biochimica et Biophysica Acta 1368 (1998) 247-255

[87] K. Kawasaki, Phys. Rev. 150291 (1966)

[88] V. S. Kononenko, Sov. Phys. Acoust. 31 (1985) 499

[89] B. A. Korgel, J. H. Zanten, H. G. Monbouquette, Biophys. J. 74 (1998) 32643272

[90] J. Korlach, P. Schwille, W. W. Webb, G. W. Feigenson, Biophysics 96 (1999) 8461-8466

[91] D. M. Kroll, J.M. Ruhland, Phys. Lett. 80A (1980) 45

[92] D. M. Kroll, J.M. Ruhland, Phys. Rev. A23 (1981) 371

[93] A. Labhardt, Dissertation, Phil.-Nat.-Fak. Univ. Basel (1975)

[94] A. Labhardt, G. Schwarz, Ber. Bunsges. Phys. Chem. 80 (1973) 83

[95] Z. I. Lalchev, A. R. Mackie, Collods and Sourfaces B: Biointerfaces 15 (1999) $147-160$

[96] K. Lautscham, Dissertation, Universität Göttingen (1986)

[97] K. Lautscham, F. Wente, W. Schrader, U. Kaatze, Meas. Sci. Technol. 11 1-8 (2000)

[98] D. W. Marquardt, J. Soc. Indust. Appl. Math. 2 (1963)

[99] D. Marsh, Handbook of Lipid Bilayers, Boston (1990)

[100] V. M. Maynard, R. L. Magin, F. Dunn, Chemistry and Physics of Lipids 37 (1985) 1-12

[101] T. P. McMullen, R. N. A. H. Lewis, R. N. McElhaney, Biochemistry 32 (1993) 516-522

[102] T. P. McMullen, R. N. McElhaney, Biochim. Biophys. Acta, 1234 (1995) 90-98

[103] K. Menzel, Dissertation, Mat.-Nat.-Fak. Univ. Göttingen (1993)

[104] E. Meyer, D. Guicking, Schwingungslehre, Vieweg-Verlag (1974)

[105] E. Meyer, E. G. Neumann, Physikalische und technische Akustik, Vieweg Braunschweig (1979)

[106] S. Mitaku, A. Ikegami, A. Sakanishi, Biophysical Chemistry 8 (1978) 295-304 
[107] S. Mitaku, Mol. Cryst. Liq. Cryst. 70 (1981) 21-28

[108] S. Mitaku, T. Date, Biochimica et Biophysica Acta 688 (1982) 411-421

[109] S. Mitaku, T. Jippo, R. Kataoka, Biophys. J. 42 (1983) 137-144

[110] R. Morse, L. D. Ma, R. L. Magin, F. Dunn, Chemistry and Physics of Lipids 103 (1999) 1-10

[111] O. G. Mouritsen, Chem. Phys. Lipids 57 (1991) 179-194

[112] O. G. Mouritsen, K. Jørgensen, Chem. Phys. Lipids 73 (1994) 3-25

[113] L. K. Nielsen, T. Bjornholm, O. G. Mouritsen, Nature 404 (2000) 352

[114] Y. Nishizuka, Science 233 (1984) 305-311

[115] W. Notling, Statistische Physik, Band 6 der Reihe Grundkurs: Theoretische Physik. Zimmermann - Neufang, Ulmen, 1. Auflage, 1994

[116] J. C. Owicki, H. M. McConnell, Biophys. J. 30 (1980) 383-398

[117] P. Petong, Diplomarbeit, Mat.-Nat.-Fak. Univ. Göttingen (1996)

[118] M. C. Phillips, D. Chapman, Biochim. Biophys. Acta 163 (1968) 301

[119] K.S. Pitzer, J. Chem. Phys. 12 (1944) 310

[120] R. Pottel, K.-D. Göpel, R. Henze, U. Kaatze, Volmar Uhlendorf, Biophysical Chemistry 19 (1984) 233-244

[121] R. Pottel, Dielektrische Relaxation und molekulare Korrelationszeit, Beitrag zur Sommerschule "Physik des flüssigen Zustandes", St. Georgen/Längsee/Kärnten, (12. bis 23.9.77), 182-185

[122] V. P. Romanov, V. A. Solovjev, Sov. Phys. Acoust. 1168 (1965)

[123] V. P. Romanov, V. A. Solovjev, Sov. Phys. Acoust. 11 (1965) 219

[124] P. Rouse, J. Chem. Phys. 21 (1953) 1272

[125] A. Rupprecht, Diplomarbeit, Mat.-Nat.-Fak. Univ. Göttingen (1994)

[126] E. Sackmann, Ber. Bunsenges. Phys. Chem. 78 (1974) 9

[127] E. Sackmann, Physikalische Grundlagen der molekularen Organisation und Dynamik von Membranen, Biophysik 2. Auflage, Herausgeber: Walter Hoppe, Wolfgang Lohmann, Hubert Martel, Hubert Ziegler, Springer-Verlag (1982), 439-475

[128] E. Sackmann, Physical basis of self-organisation and function of membranes: physics of vesicles, In: A. J. Hoff, R. Lipowsky, E. Sackmann (Herausgeber) Structure and dynamics of membranes: from cells to vesicles Band 1 A der Reihe Handbook of biological physics Kapitel 5, 213-304, Elsevier Science B.V., 1. Auflage 1995

[129] A. P. Sarvazyan, Annu. Rev. Biophys. Biophys. Chem. 20 (1991) 321-342

[130] K. Schorn, Dissertation, Mat.-Nat.-Fak. Univ. München (1996) 
[131] W. Schrader, Diplomarbeit, Mat.-Nat.-Fak. Univ. Göttingen (1998)

[132] P. Schwille, J. Korlach, W. W. Webb, Cytometry 36 (1999) 176-182

[133] K. Semmler, H. W. Meyer, P. J. Quinn, Chemistry and Physics of Lipids 99 (1999) 155-167

[134] K. Simons, E. Ikonen, Nature 387 (1997) 569-572

[135] P. R. Strom-Jensen, R. L. Magin, F. Dunn, Biochimica et Biophysica Acta 769 (1984) 179-186

[136] L. Stryer, Biochemie, Spektrum Adakemischer Verlag Heidelberg, 2. Auflage 1994

[137] I. P. Sugár, R. L. Biltonen, N. Mitchard, Methods In Enzymology 40 (1994) 569-593

[138] H. Tanaka, Y. Wada, Phys. Rev. A 32 (1985) 512

[139] K. M. G. Taylor, R. M. Morris, Thermochimica Acta 248 (1995) 289-301

[140] T. Telgmann, Dissertation, Mat.-Nat.-Fak. Univ. Göttingen (1997)

[141] A. V. Tobolsky, D. B. DuPre, Journal of Polymer Science A-2 6 (1968) 11771182

[142] H. Träuble, D. Haynes, Chem. Phys. Lip. 7 (1971)

[143] W. L. C. Vaz, E. C. C. Melo, T. E. Thompson, Biophys. J. 56 (1989) 869-876

[144] W. L. C. Vaz, F. Goodsaid-Zalduondo, K. Jacobson, FEBS Letters 174 (1984) 2

[145] W. L. C. Vaz, R. M. Clegg, D. Hallmann, Biochemistry 24 (1985) 781-786

[146] W. L. C. Vaz, Z. I. Derzko, K. A. Jacobson, Membrane Reconstitution, Editors: G. Poste, G. L. Nicolson, (1982) 83-136

[147] K. Wójtowicz, W. I. Gruszecki, M. Walicka, J. Barwicz, Biochimica et Biophysica Acta 1373 (1998) 220-226

[148] F. Wente, Diplomarbeit, Mat.-Nat.-Fak. Univ. Göttingen (1998)

[149] M. Wortis, M. Jarić, U. Seifert, Journal of Molecular Liquids 71 (1997) 195-207

[150] L. L. M. van Deenen, U. M. T. Houtsmuller, G. H. Haas, E. Mulder, J. Pharmacy Pharmacol. 14 (1962) 429

[151] P. W. M. van Dijck, A. J. Kaper, H. A. J. Oonk, J. de Gier, Biochim. Biophys. Acta 470 (1977) 58 


\section{Danksagung}

Herrn Prof. Dr. D. Ronneberger danke ich für die Betreuung dieser Arbeit.

Ganz herzlich möchte ich an dieser Stelle Herrn Dr. U. Kaatze danken, der wie kein anderer zu jeder Zeit zu Diskussionen bereit war und mich in jeglicher Hinsicht bei dieser Arbeit unterstützt hat.

Ebenfalls gilt mein Dank Herrn Dr. R. Behrends für die anregenden und förderlichen Diskussionen. Ich danke auch allen Mitarbeitern der Arbeitsgruppe für die konstruktive Zusammenarbeit und die angenehme Arbeitsatmosphäre.

Bei Herrn Dr. K. Lautscham, Herrn D. Hille und allen anderen Mitarbeitern der elektronischen und feinmechanischen Werkstatt bedanke ich mich für Ihren Einsatz bei der Entwicklung und Wartung der Meßapparaturen. Mein besonderer Dank gilt an dieser Stelle Herrn Dr. K. Lautscham für seinen selbstlosen Einsatz bei der Entwicklung der Schallgeschwindigkeitszelle.

Herrn Dr. T. Heimburg danke ich an dieser Stelle für die freundliche Bereitsstellung des Kalorimeters zur Messung der Wärmekapazitätskurven und für die sich daraus ergebenden Diskussionen.

Frau M. Höckel danke ich für die Bereitstellung zahlreicher Ultraschallspektren und Wärmekapazitätskurven.

Herrn S. Halstenberg danke ich für die Bereitstellung zahlreicher Ultraschallspektren und einiger Schallgeschwindigkeitskurven.

Herrn F. Wente danke ich für die Bereitstellung einiger Schallgeschwindigkeitskurven.

Herrn M. Kahle danke ich für die Bereitstellung einiger Wärmekapazitätskurven.

Herrn Prof. Dr. J. K. Bhattacharjee danke ich für sein Interesse an meiner Arbeit und für die zahlreichen Beträge zur Diskussion.

Meinen Eltern danke ich für der Ermöglichung des Physikstudiums.

Frau Maren Kirschke gilt mein besonderer Dank.

Diese Arbeit wurde von der Deutschen Forschungsgemeinschaft finanziell unterstützt. 


\section{Lebenslauf}

Name:

Geburtsdatum:

Geburtsort:

Staatsangehörigkeit:

Familienstand:

08/79-07/83

08/83-06/92

$06 / 92$

08/92-10/93

$10 / 93$

$10 / 95$

06/96-08/96

$10 / 98$

10/98-04/99

ab $01 / 99$
Wilfried Schrader

13. Oktober 1972

Warburg

deutsch

ledig

Grundschule der Samtgemeinde Boffzen

König-Wilhelm-Gymnasium in Höxter

Abitur

Zivildienst in der Jugendherberge Höxter

Beginn des Studiums Physik an der GeorgAugust-Universität in Göttingen

Diplomvorprüfung im Fach Physik

zweimonatiges Praktikum bei der Firma Polytec PI in Waldbronn; Weiterentwicklung einer lasergeführten Roboterkopfsteuerung

Diplomprüfung. Das Thema der Diplomarbeit, die am Dritten Physikalischen Institut unter der Leitung von Dr. U. Kaatze angefertigt wurde, lautete: Dielektrische Spektroskopie an wäßrigen Suspensionen ionischer Phospholipid-Vesikel

Wissenschaftliche Hilfskraft zur Ausrichtung der Übungen zur Vorlesung Mathematik \& Statistik I, II für Agrarwissenschaftler im Fachbereich Agrarwissenschaften der Universität Göttingen

Wissenschaftlicher Angestellter am Dritten Physikalischen Institut und Beginn der Dissertation mit dem Thema: Fluktuationen in Membranen -Schallgeschwindigkeitsmessungen, Kalorimetrie sowie dielektrische und akustische Spektroskopie an wäßrigen Phospholipidsuspensionen 